University of Massachusetts Amherst

ScholarWorks@UMass Amherst

Doctoral Dissertations 1896 - February 2014

$1-1-2002$

\title{
Miktoarm star block copolymers : effects of molecular architecture on morphology.
}

Lizhang, Yang

University of Massachusetts Amherst

Follow this and additional works at: https://scholarworks.umass.edu/dissertations_1

\section{Recommended Citation}

Yang, Lizhang, "Miktoarm star block copolymers : effects of molecular architecture on morphology." (2002). Doctoral Dissertations 1896 - February 2014. 1028.

https://doi.org/10.7275/p3zf-ms44 https://scholarworks.umass.edu/dissertations_1/1028

This Open Access Dissertation is brought to you for free and open access by ScholarWorks@UMass Amherst. It has been accepted for inclusion in Doctoral Dissertations 1896 - February 2014 by an authorized administrator of ScholarWorks@UMass Amherst. For more information, please contact scholarworks@library.umass.edu. 
MIKTOARM STAR BLOCK COPOLYMERS:

EFFECTS OF MOLECULAR ARCHITECTURE ON MORPHOLOGY

A Dissertation Presented

by

LIZHANG YANG

Submitted to the Graduate School of the

University of Massachusetts Amherst in partial fulfillment of the requirements for the degree of

DOCTOR OF PHILOSOPHY

February 2002

Polymer Science and Engineering 
(C) Copyright by Lizhang Yang 2002

All Rights Reserved 


\section{MIKTOARM STAR BLOCK COPOLYMERS:}

EFFECTS OF MOLECULAR ARCHITECTURE ON MORPHOLOGY

A Dissertation Presented

by

\section{LIZHANG YANG}

Approved as to style and content by:

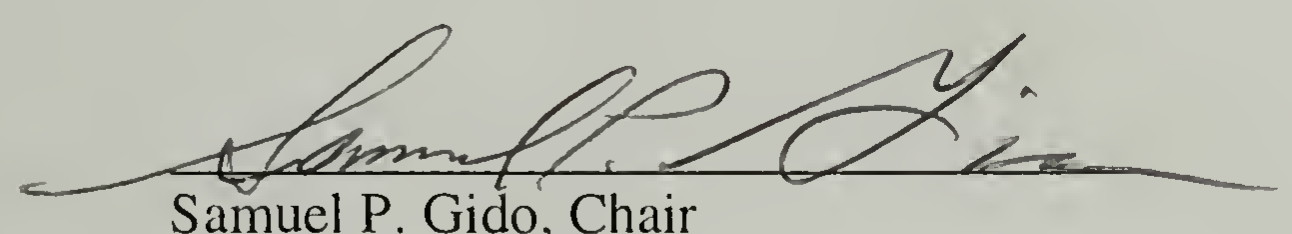

Samuel P. Gido, Chair

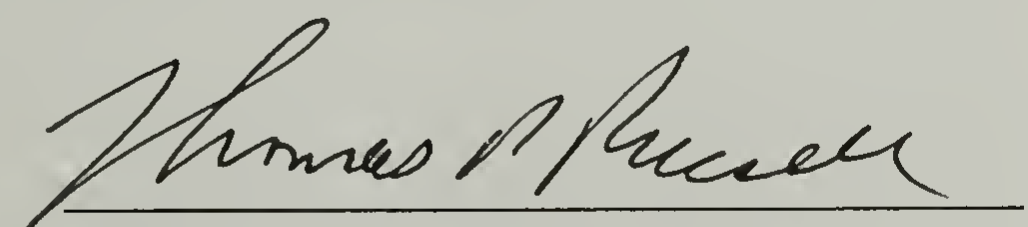

Thomas P. Russell, Member

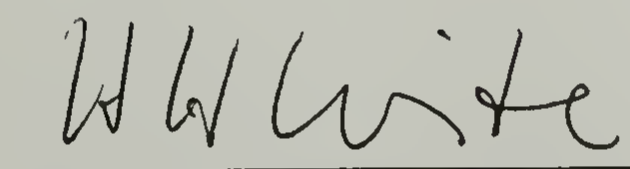

H. Henning Winter, Member

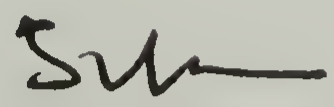

Thomas J. McCarthy, Head

Polymer Science \& Engineering 
To my parents, Xi-yuan Yang and Cheng-rong Yan, for their love and support. 


\section{ACKNOWLEDGEMENTS}

Much of the credit for my current level of academic achievement belongs to the educators I've encountered over the course of the last 22 years. A few names come immediately to mind. English teacher Ming-qin Wang of Ping-ding-shan $1^{\text {st }}$ middle school taught me how easy studying can be. As the result, my scores jumped from the lowest to number one in my class; Chemistry teacher Yu-an Li of Henan Province Experiment High School taught me how to set up a goal and pursue it, a skill crucial for my development. Professor Jimmy W. Mays of the University of Alabama, Birmingham, took an active interest in my graduate career at an early stage, and has provided me with valuable experience. It has been my distinct pleasure to work with both Prof. Thomas P. Russell and Prof. H. Henning Winter of UMass during the course of my dissertation research, and I owe them thanks for their valuable time and input.

I must also pay due credit to those who have contributed formally to the studies described in this manuscript. From the University of Alabama at Birmingham, David Uhrig, Kunlun Hong, Stergios Pispas, and Prof. Jimmy Mays contributed invaluable time and effort in the realm of sample synthesis, and I am privileged to count them also as friends. From the University of Athens, Greece, Gabriel Velis, and Prof. Nikos Hadjichristidis also contributed greatly to the synthetic this work. Finally, Dr. Roland Weidisch of Martin-Luther Universität Halle Wittenberg deserves much credit for their suggestions and help. 
It is such a pleasure and privilege to work in this department. Darrin, Chin, and Rick helped me on the initial stages of my research; Sheng gave me many excellent suggestions. Louis helped me greatly on Microscopy support; John helped me design and construct Roll Caster; Eileen, Sophia, and many other secretaries make the life around us easy. The janitors keep the place neat and clear. It is so enjoyable to work in such a place.

Finally and certainly not least among my academic colleagues, I am indebted to my dissertation advisor, Prof. Samuel P. Gido. Without doubt I have learned much from Sam, sometimes not of my choice, but always to my advantage. Sam has taught me much about scientific research, block copolymers, and the many practical matters, which will surely serve me well in the future.

I have been lucky to count many among my friends during the last four years, but a handful of these I know will be pestering me for years to come. Sheng helped me keep the sanity as we "compliment" each other. Susan, Lisa and John helped me to learn how difficult English can be. Xiaodong, Yong, and Chuntao showed me how much fun Poker Games could be in a hot summer evening. Tao Xie has been great for showing me how colorful life is in Umass. Kunlun, David, and Jinchun taught me the how versatile glasses were. True friends are rare, and I am fortunate to have so many.

To my family, I owe a debt I cannot express much less repay. Without fail, they have always been supportive of my academic undertakings; my success has been a family project from cradle through the current stage. Especially, I want to thank my great grant 
father, Shi-gui Yang. He was a wise man and valued education. He sent children to school despite all the difficulties. His third son was the first university graduate in my hometown; my father was the second; and I am becoming the first Ph.D. of the town.

Finally, I would like to thank my wife Xiao Xie. Her love is true blessing having no explanation nor requiring one. Our relationship has become the foundation on which I've stood during the past five years. Her love has made me a better person, and that may be the best result of my time in graduate school. 
ABSTRACT

MIKTOARM STAR BLOCK COPOLYMERS:

EFFECTS OF MOLECULAR ARCHITECTURE ON MORPHOLOGY

FEBRUARY 2002

LIZHANG YANG, B.S., BEIJING UNIVERSITY

M.S., UNIVERSITY OF MASSACHUSETTS AMHERST

PH.D., UNIVERSITY OF MASSACHUSETTS AMHERST

Directed by: Professor Samuel P. Gido

Three aspects of the effects of molecular architecture on block copolymer and block copolymer / homopolymer blends morphological behavior have been investigated. In the Chapter 2 and 3, the morphological behaviors of "Model" graft block copolymer / homopolymer blends was discussed. The morphological behaviors for two distinct types of graft architectures were investigated. The first type, $\mathrm{I}_{2} \mathrm{~S}$ block copolymers, which have 2 low polydispersity (PDI) polyisoprene arms and one low PDI polystyrene arm joint at a single junction point, has an asymmetry, non-linear molecular architecture. The second type, $\mathrm{I}_{2} \mathrm{~S}_{2}$ block copolymers, which have 2 low PDI polyisoprene arms and two low PDI polystyrene arms joint at a single junction point, has a symmetry, non-linear molecular architecture. In the blend study, a slow co-casting procedure was developed to get single crystal structure of Gyroid morphology. The amazing scattering patterns of this sample provide the best evidence for Gyroid morphology observed so far. 
In Chapter 4, morphological behavior of $\mathrm{I}_{5} \mathrm{~S}$ block copolymers was studied to investigate the systematic discrepancies between the theoretical predicated miktoarm star block copolymer morphology and the experimental observations. The current results indicate that geometrical packing constraints prevent the formation of morphologies such as spheres and cylinders in highly asymmetric miktoarm stars in which the minor volume fraction component would need to occupy the matrix phase. Unusual broken chevron tilt grain boundary morphologies were also observed in a lamellar $\mathrm{I}_{5} \mathrm{~S}$ material. We attribute these new structures to the asymmetric energy penalties for interfacial bending which result from the molecular asymmetry of the miktoarm stars.

Finally, irreversible morphology transformation from lamella to cylinder was investigated using selective solvent approach. Selective solvent can preferentially swell one of the components of block copolymers, increase the effective volume fraction of that component during solvent casting and thus affect the resulting block copolymer morphology. During the subsequent annealing, the kinetically trapped unstable morphology will transform to its stable morphology. By controlling the annealing temperature and the length of annealing time, we will be able to tract the detailed information about the morphology transformations. 
ACKNOWLEDGEMENTS

ABSTRACT viii

LIST OF TABLES xii

LIST OF FIGURES xiii

\section{CHAPTER}

1. INTRODUCTION

$1.1 \quad$ Miktoarm Stars ................................................................................ 1

1.2 "Model" Graft Stars block copolymer / homopolymer blends .................... 2

1.3 Block copolymer morphology transformations........................................ 3

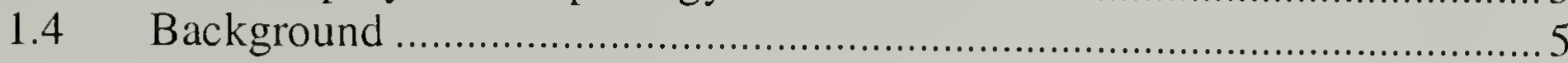

1.4.1 Self-Assembly of Linear Diblock Copolymers ................... 5

1.4.2 The Milner Model ….................................................. 8

2. MORPHOLOGICAL BEHAVIOR OF $\mathrm{I}_{2} \mathrm{~S}$ SINGLE GRAFT BLOCK COPOLYMER / HOMOPOLYMER BLENDS

2.1 Abstract …

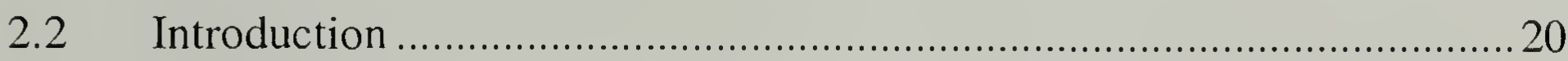

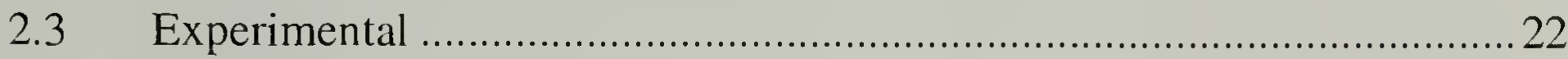

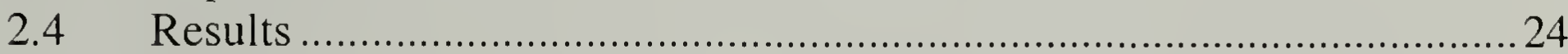

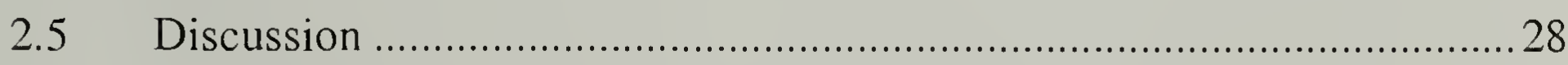

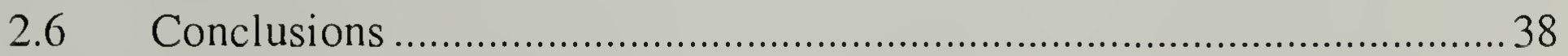

3. MORPHOLOGY BEHAVIOR OF $X$ SHAPED SINGLE GRAPHED BLOCK COPOLYMER / HOMOPOLYMER BLENDS ..............................66

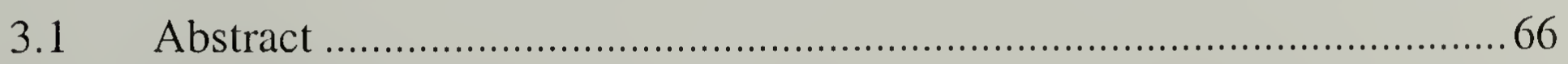

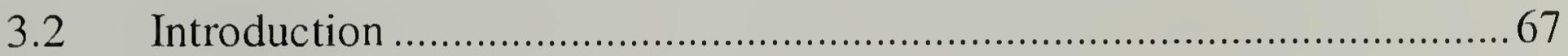

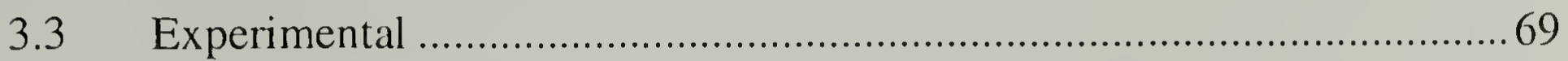

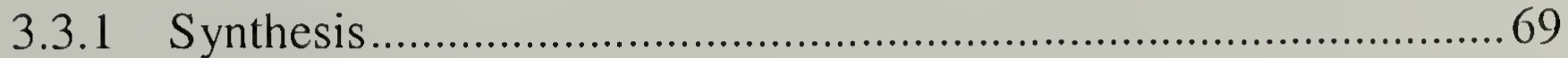

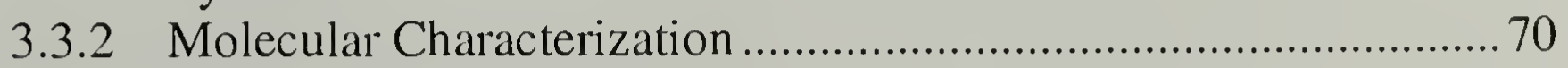

3.3.3 Morphological Characterization ................................................. 71

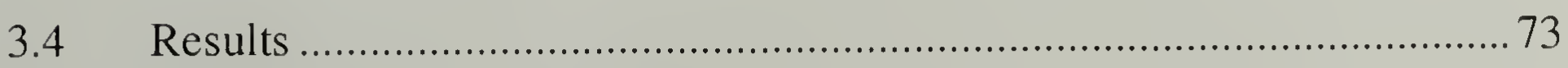

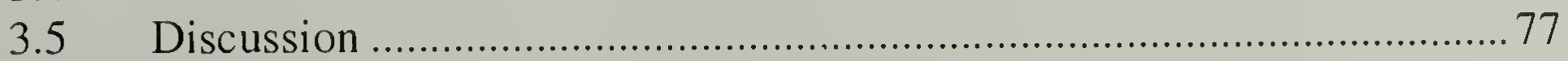


4. MORPHOLOGICAL BEHAVIOR $\mathrm{I}_{5} \mathrm{~S}$ MIKTOARM STAR BLOCK COPOLYMERS:PACKING CONSTRAINTS ON MORPHOLOGY AND DISCONTINUOUS CHEVRON TILT GRAIN BOUNDARIES ...... 106

4.1 Abstract

4.2 Introduction

Experimental

4.5

Results

Discussion

Conclusions

5. MORPHOLOGICAL TRANSFORMATION FROM LAMELLA TO CYLINDER

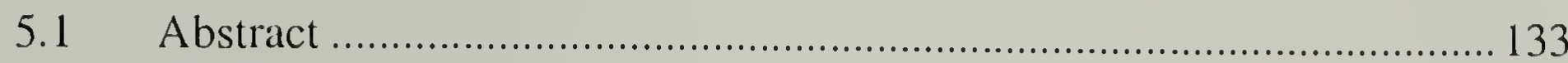

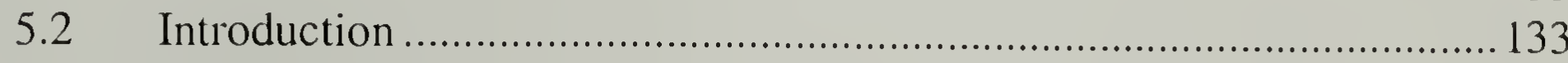

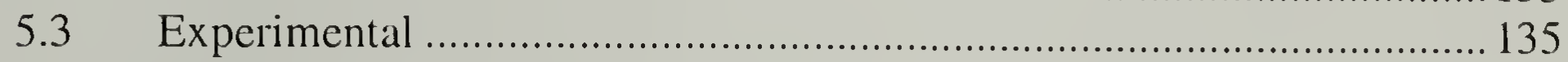

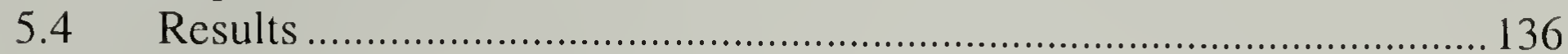

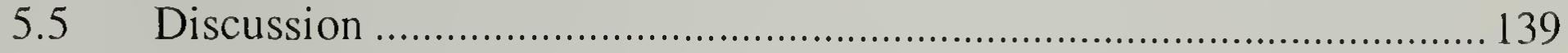

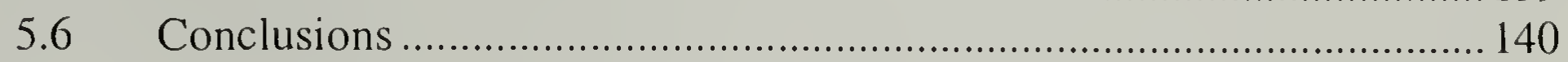

6. CONCLUSIONS AND FUTURE WORK

6.1 Miktoarm Star Block Copolymers: the Milner Model and the broken tilt lamella grain boundaries.

6.1.1 Proposed Research

6.2 Investigation of morphology transformations using selective solvent approach.

6.2.1 Proposed research

6.3 Morphology of Miktoarm star block copolymer / homopolymer blends.

6.3.1 Proposed Research 156 


\section{LIST OF TABLES}

Table

2.1. Molecular characterization of the $\mathrm{I}_{2} \mathrm{~S}$ block copolymer and the PS and PI homopolymers.

2.2. Composition and Morphology of the blends in the study 40

2.3. Table of brush heights and swelling ratios of the blend samples.

3.1. Molecular characterization of the $\mathrm{I}_{2} \mathrm{~S}_{2}$ block copolymer and the PS and PI homopolymers.

3.2. Compositions and Morphologies of the blends in the study

3.3. Observed reflections their intensities.

3.4. The permitted reflections of $I a \overline{3} d$ and observed reflections for IX35-30 .84

4.1. Molecular and morphological characterization information for the three $\mathrm{I}_{5} \mathrm{~S}$ miktoarm stars and the dumbbell shaped $\mathrm{I}_{5}-\mathrm{S}-\mathrm{I}_{5}$ graft block copolymer 


\section{LIST OF FIGURES}

Figure

1.1. Schematic of an $\mathrm{A}_{3} \mathrm{~B}_{2}$ miktoarm star comprised of three arms of polymer $\mathrm{A}$ and two arms of polymer $B$.

1.2. Strong segregation regime morphology diagram generated using the Milner model

1.3. Schematics of morphology transformations.

1.4. Morphology diagram for a linear AB diblock copolymer calculated by Matsen and Bates, spanning from the order-disorder transition into the strong segregation regime.

1.5. Gaussian wedges for lamellar, cylindrical, spherical, and OBDD bicontinuous morphologies used in the Milner model for calculating morphological behavior of miktoarm star block copolymers.

1.6. Strong segregation regime morphology diagram generated using the Milner model.

2.1. Schematic of an $\mathrm{I}_{2} \mathrm{~S}$ single graft block architecture with one PS arms (dashed) and two PI arms (solid).

2.2. Isotropic SAXS patterns of SY62 series blends azimuthally integrated into one-dimensional plots of $\log (\mathrm{I})$ verses $\mathrm{q}$.

2.3. TEM images of blend SY62-82.

2.4. SY62 blend series mapped onto the theoretical morphology diagram.

2.5. S81 and IY81 blend series mapped onto the theoretical morphology diagram....46

2.6. TEM micrographs of blends in the SY81 series.....

2.7. Isotropic SAXS patterns of SY81 series samples azimuthally integrated to give plots of $\log (\mathrm{I})$ verses $\mathrm{q}$.

2.8. TEM micrographs from the IY81 series

2.9. Isotropic SAXS patterns of IY81 series samples azimuthally integrated to give plots of $\log (\mathrm{I})$ verses $\mathrm{q}$.

2.10. TEM image of IY89 series.

2.11. TEM tilt series of IY89-82. 56 
2.12. Vesicle model

2.13. Plot of asymmetry swelling ratio, $\mathrm{K}$, verses homopolymer volume fraction for the miktoarm block copolymer / homopolymer blends of this study and the linear diblock / homopolymer blends studies by Winey, Thomas, and Fetters ${ }^{19}$

2.14. Hexagonally perforated lamella model.

2.15. The schematic and the TEM micrograph when microtome is parallel to $X Y$ plane.

2.16. The schematic and the TEM micrograph when microtome is perpendicular to $\mathrm{XY}$ plane.

2.17. The schematic and the TEM micrograph when microtome direction has an angle with XY plane.

3.1. Illustrations of $\mathrm{A}_{2} \mathrm{~B}_{2}$ miktoarm star block copolymer architecture. 86

3.2. Morphology diagram with $\mathrm{A}_{2} \mathrm{~B}_{2}$ block copolymer sample positions indicated.... 87

3.3. $\log (\mathrm{I})$ vs. q SAXS patterns for the SXI4 blend series 88

3.4. Blend series SX14 mapped onto the theoretical morphology diagram.................89

3.5. $\log (\mathrm{I})$ vs. q SAXS patterns for the SX14 blend series ....................................... 90

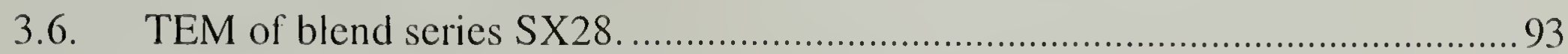

3.7. Blend series SX28 mapped onto the theoretical morphology diagram................94

3.8. $\log (\mathrm{I})$ vs. q SAXS patterns for the IX68 blend series ......................................95

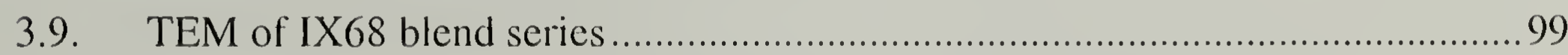

3.10. Blend series IX68 mapped onto the theoretical morphology diagram............... 100

3.11. $\log (\mathrm{I})$ vs. q SAXS patterns for the IX68 blend series .................................... 101

3.12. Two dimensional single crystal SAXS pattern of IX35-30 gyroid structure...... 103

3.13. TEM of IX35-32

3.14. Blend series IX35 mapped onto the theoretical morphology diagram............... 105

4.1. Illustration of the molecular architecture of an $I_{5} \mathrm{~S}$ miktoarm star bleck copolymer and dumbbell shaped $\mathrm{I}_{5}-\mathrm{S}-\mathrm{I}_{5}$ graft block copolymer.... 
4.2. $\log (\mathrm{I})$ vs. q $\mathrm{SAXS}$ data for the $\mathrm{I}_{5} \mathrm{~S}$ and the $\mathrm{I}_{5}-\mathrm{S}-\mathrm{I}_{5}$ samples.

4.3. Two-dimensional SAXS for sample $\mathrm{I}_{5} \mathrm{~S}-46$

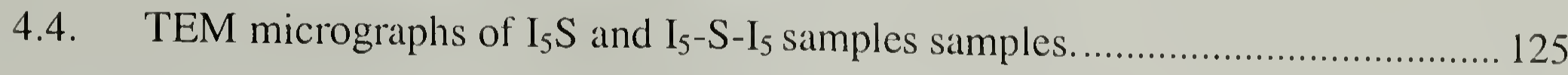

4.5. The morphology diagram generated by the Milner model............................... 126

4.6. Values of $\Delta \varepsilon$ for samples exhibiting morphologies other than those predicted by theory, as a function of $\varepsilon$, for the data shown in Figure 4.5.

4.7. $\mathrm{I}_{5} \mathrm{~S}-58$ Symmetric tilt grain boundaries.

4.8. Schematic of commonly observed chevron tilt boundaries.

4.9. (a) illustration of chevron tilt boundaries for $I_{5} S$ samples, (b) illustration of broken or discontinuous chevron tilt boundaries for $\mathrm{I}_{5} \mathrm{~S}$ samples.

5.1. Illustration of the molecular architecture of an $\mathrm{I}_{3} \mathrm{~S}$ miktoarm star block copolymer. The $\mathrm{I}_{3} \mathrm{~S}$ star is comprised of three PI arms and one PS arm joined at a single junction point

5.2. TEM micrographs of $\mathrm{I}_{3} \mathrm{~S}-86-\mathrm{B}$ and $\mathrm{I}_{3} \mathrm{~S}-86-\mathrm{C}$ :

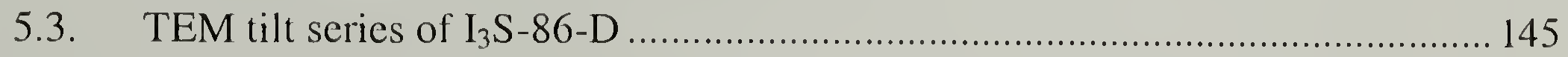

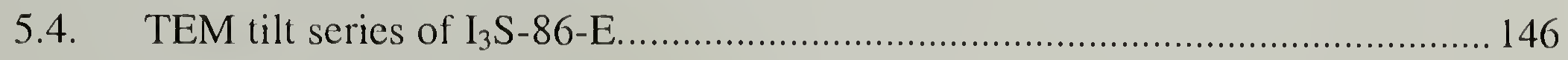

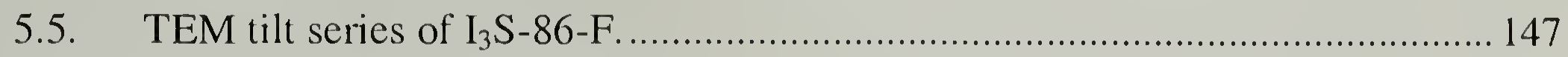

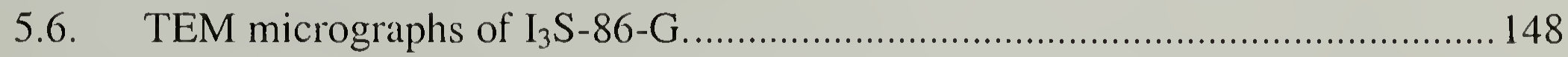

5.7. Morphology transformation sequence schematic for the lamella to cylinder transformation of $\mathrm{I}_{3} \mathrm{~S}-86$.

6.1. Top view and side view of the "roll caster". 


\section{CHAPTER 1}

\section{INTRODUCTION}

\subsection{Miktoarm Stars}

Study of the morphological behavior of block copolymers with non-linear, grafted architectures has been extensive over the last seven years. ${ }^{1-10}$ This work originated in part from an interest in finding new ways to control the formation of block copolymer mesophases other than by variations in temperature and relative volume fractions of the component materials. ${ }^{2}$ Results of an early study examining the morphological behavior of a limited set of $\mathrm{A}_{\mathrm{n}} \mathrm{B}_{\mathrm{m}}$-type stars indicated that molecular architecture could significantly affect morphological behavior. ${ }^{1}$ Figure 1.1 illustrates a model $A_{n} B_{m}$-type block copolymer, in this case having $n=3$ and $m=2$. This work was facilitated by developments in chlorosilane chemistry techniques, which allowed the coupling of blocks of living polymers in new ways. ${ }^{1}$

In 1994, Milner presented a mean-field model predicting the combined effects of architectural asymmetry, a difference in the number of arms of components $\mathrm{A}$ and $\mathrm{B}$, and conformational asymmetry, the difference in space-filling characteristics of components $\mathrm{A}$ and $\mathrm{B}$, on the morphological behavior of $\mathrm{A}_{\mathrm{n}} \mathrm{B}_{\mathrm{m}}$-type stars. ${ }^{11}$ This work provided a guide to experimentally examine the morphological behavior of these materials, named "miktoarm" stars by Hadjichristidis. Miktoarm is a combination of the Greek $\mu \iota \kappa \tau o \sigma$, meaning "mixed", and arm. Both miktoarm star and $\mathrm{A}_{\mathrm{n}} \mathrm{B}_{\mathrm{m}}$-type star will be used through out this work to refer to block copolymers with this architecture. 
The Milner model was relatively successful in predicting the morphological behavior of miktoarm stars with relatively low architecture asymmetry. ${ }^{29,12}$ However, samples with relatively high architecture asymmetry were found to exhibit morphological behaviors differing from the predictions of the Milner model. ${ }^{8}$ These discrepancies are shown in Figure 1.2. These discrepancies suggested that while the model correctly predicted the basic effects of architecture on morphological behavior, the specific details of the miktoarm star molecular architecture and its impact on morphology might not be properly described. The previous experimental studies at high asymmetry parameter did not indicate the morphology transformation volume fractions. Thus, a further study with high molecular asymmetry miktoarm stars to show exact morphology transformation is important, so that we will be able to see the degree of agreement between the theoretically calculated morphology diagram with the experimental data, and find the reasons for the observed discrepancies.

1.2 "Model" Graft Stars block copolymer / homopolymer blends

Linear diblock copolymer / homopolymer blends and linear multi block copolymer / homopolymer blends systems have been extensively studied. ${ }^{13-25}$ Previously reported linear diblock copolymer / homopolymer blend results showed that the OOTs in the blends occur at about the same volume fractions as those of pure linear diblock copolymers. ${ }^{17,18,20}$ However, for architecturally asymmetric graft copolymers, blending homopolymer into the one arm side or into the multi-arm side of the interface may produce different morphological results. For architecturally symmetric yet non-linear 
star shaped block copolymers, blending homopolymer may not produce the same morphological behavior as that of linear block copolymers. In the present study, $\mathrm{I}_{2} \mathrm{~S}$ block copolymers and $\mathrm{I}_{2} \mathrm{~S}_{2}$ block copolymers are blended with homopolymers of polystyrene (PS) or homopolymer polyisoprene (PI) to investigate their morphological behaviors. Blends of graft block copolymers and homopolymers are used widely in industrial applications where graft copolymers are used as compatibilizers to control the microphase separated morphology and thus the properties of blends. ${ }^{26-30}$ The complex graft block copolymers can be viewed as a combination of their structure units, which are usually $\mathrm{A}_{2} \mathrm{~B}_{2}$ and $\mathrm{A}_{2} \mathrm{~B} \cdot{ }^{31}$ Therefore, it important to investigate to blends behavior of "model" $\mathrm{A}_{2} \mathrm{~B}, \mathrm{~A}_{2} \mathrm{~B}_{2}$ star shaped lock copolymer homopolymer blends.

\subsection{Block copolymer morphology transformations}

Despite considerable interest in equilibrium diblock copolymer phase behaviors, the mechanisms of transitions between different morphologies have received comparatively little attention. However, an understanding of morphology transformation processes has implications for practical of morphology control. Both experimental and theoretical data indicate that in the vicinity of the order-disorder transition, phase boundaries acquire significant curvature in the composition (f) -segregation $(\chi N)$ plane, and the system becomes thermotropic due to the temperature dependence of $\chi$. This kind of heat transformation is usually thermal reversible and has been the focus of many recent studies. ${ }^{32-40}$ The schematic of this kind of transformations are shown in Figure 1.3. Since temperature can only affects $\chi \mathrm{N}$ in a certain range, low molecular weight block 
copolymers are ustally used, and these low MW polymers have fast kinetics at elevated temperatures. Temperature affects both morphology and the kinetics, making it difficult to deconvolute these two factors. At high $\chi N$ value, order-order transition phase boundaries become nearly independent of segregation. In this regime, non-cquilibrium morphologies can be prepared though casting from a selective solvent and their relaxation can be followed. The schematic is also shown in Figure 1.3. Selective solvent can preferentially swell one of the components of block copolymers, increase the effective volume fraction of that component cluring solvent casting and thus affect the resulting block copolymer morphology. During the subsequent annealing, the kinetically trapped unstable morphology will transform to its stable morphology. By controlling the annealing temperature and the length of annealing time, the detailed information about the morphology transformations can be tracked. This kind of morphology transformation is not reversible. Comparing with heat transformations, selective solvent approach to study morphology has many advantages: First, higher molecular weight polymers can be used; Second, wider range of molecular composition can be used, since we can adjust the effective volume fraction of the system by choosing different selective solvents; Third, at these high $\chi \mathrm{N}$ range, the order-order transition is nearly unaffected by changes in segregation, and temperature only affects the kinetics, thus deconvolute kinetics and thermodynamics is not necessary; Finally, it is also possible to get accurate information about morphology-property relationships, since different morphologies can be achieved from the same material and high MW polymer, which is a requirement for many mechanical testing, can be used. 
The following sections are offered as a brief introduction to the basic principles applied in this dissertation. The reader is advised to refer to the cited references for a more complete discussion of these topics.

\subsubsection{Self-Assembly of Linear Diblock Copolymers}

The mechanism for self-assembly of linear (AB) diblocks into ordered mesophases derives from the basic tendency of immiscible systems to phase separate when unfavorable enthalpic interactions overcome the entropic benefits of mixing. ${ }^{41}$ In block copolymers, however, the joining of immiscible blocks into one molecule prevents conventional, macroscopic phase separation. Instead, the driving force to minimize unfavorable A-B contacts produces a system that forms mesomorphic structures on the nanoscopic level. ${ }^{42,43}$

A variety of interesting morphologies are formed when block copolymers selfassemble. These include alternating lamellae of $\mathrm{A}$ and $\mathrm{B}$, cylinders of one component in a matrix of the other, and spheres of one component in a matrix of the other. Other morphologies that have been observed include cubic bicontinuous morphologies, perforated lamellae, and undulating lamellae.

A microphase separated, bicontinuous, block copolymer structure with a cubic symmetry was identified as ordered bicontinuous double diamond (OBDD) with Pn3m symmetry by Thomas and coworkers, ${ }^{44}$ and the Hashimoto group,${ }^{45}$ in the mid-1980's. In 
1994, Thomas and co-workers ${ }^{46}$ and Bates and co-workers ${ }^{38}$ amended the structure of the cubic bicontinuous morphology, proposing that it was actually a gyroid structure with $I a \overline{3} d$ symmetry. SAXS evidence for the gyroid structure was provided by powder patterns with up to 13 reflections, ${ }^{38}$ and single crystal scattering patterns with up to 10 reflections. ${ }^{37-39,47}$

Hexagonally perforated lamellae, which resemble the cantenoid-lamellar structure derived by periodic area-minimizing surfaces calculations, ${ }^{48}$ were also observed recently in linear diblock systems $s^{35,37-39,46,49-52}$ in a narrow volume fraction region between HEX and LAM and in block copolymer / homopolymer blends. ${ }^{22,53,54}$ Controversy exists regarding some structure details of the perforated lamella structure and the stability of the morphology. Both $\mathrm{ABC}$ stacking of the hexagonally perforated layers ${ }^{38,52}$ and a combination of an $\mathrm{ABC}$ and $\mathrm{AB}$ stacking ${ }^{39}$ were suggested for the perforated lamellar morphology. The experimental studies by Hajduk et.al. suggest that perforated lamella is an unstable morphology involved in lamella to Gyroid transition. However, in a recent study by Yang, Gido and coworkers, perforated lamella was observed in a solvent cast blend sample of graft block copolymer and homopolymer, and the morphology persisted during thermal annealing. These observations suggest that perforated lamella can be stable or meta-stable. Using the methods of Semenov ${ }^{55}$ to estimate the free energy of perforated lamella in the strong segregation limit, Fredrickson ${ }^{56}$ showed that the perforated lamella is metastable with respect of cylinders and lamella at $\phi=0.35$. Qi and Wang $^{57-59}$ showed that the perforated lamellae appear as a "pseudostable" morphology 
during the lamellar to cylinder transition. However, this prediction has not been proved experimentally.

The selection of morphology is based simply on the free encrgy of the system. The total frec encrgy of a block copolymer system which has microphase separated is a sum of competing effects, the energy requircd to create a surface between microphase separated domains, and the energy resulting from the stretching of the polymer chains away from the newly formed interface.

Figurc 1.4 shows a morphology diagram calculated by Matsen and Bates. ${ }^{60}$ This diagram predicts $\mathrm{AB}$ diblock copolymer morphology as a function of $f$, the relative volume fraction of one component of an $\mathrm{AB}$ diblock copolymer, and the product $\chi \mathrm{N}$, where $\chi$ is the Flory-Huggins binary scgmental interaction paramcter ${ }^{61}$ and $N$ is the total degrec of polymcrization of the block copolymer. Polymers A and B in this case have identical conformational bchavior, lcading to a morphology diagram symmetric about $f=$ 0.5. This morphology diagram reflects the results of nearly 30 ycars of both thcorctical and expcrimental work toward understanding the morphological behavior of block copolymcrs, beginning with a model proposed by Helfand, ${ }^{62-72}$ and with significant refinements by Leibler ${ }^{73}$ and Semenov. ${ }^{55}$ It predicts the formation of the "classical" morphologies, alternating lamellae (L), hexagonally packed cylinders $(\mathrm{H})$, spheres $\left(Q_{1 m \underline{m} m}\right)$ on a body-centered cubic (BCC) lattice, as well as more complicated morphologies such as the "gyroid" cubic bicontinuous morphology $\left(Q_{1, \underline{1} \underline{3} \mathrm{~d}}\right) .{ }^{46,74}$ Below a critical level of $\chi \mathrm{N}=10.5$ for a block copolymer with equal volume fractions of $\mathrm{A}$ and $\mathrm{B}$, a diblock copolymer is predicted to form a homogenous, disordered morphology. 
Three regimes in Figure 1.4 are commonly defined. These are the weak segregation regime (WSR), the intermediate segregation regime (ISR), and the strong segregation regime (SSR). The divisions between these regimes are based on the change in scaling behavior of mesophase period with degree of polymerization. ${ }^{43}$ Typically, the WSR is defined by $\chi \mathrm{N}<15$, the ISR by $15<\chi \mathrm{N}<100$, and the SSR by $\chi \mathrm{N}>100$. For the purposes of most block copolymer morphology studies, samples that fall in the SSR are preferred as their behavior has been most extensively modeled and examined. In the SSR, the interface between the microphase-separated domains is a sharp division between essentially pure domains of components A and B. This condition, utilized by Helfand as the narrow interphase approximation ${ }^{(9)}$ is only possible when the unfavorable enthalpic interactions dominate the free energy of the system, such as occurs at high $\chi \mathrm{N}$.

\subsubsection{The Milner Model}

Microphase separated block copolymers have been successfully represented as polymer brushes grafted to the dividing surface between mesophases ${ }^{11,75-82}$ The model proposed by Milner applies these arguments to construct a self-consistent, mean-field theory for miktoarm star morphological behavior ${ }^{11,83}$ The free energy of a mesophase is calculated for a single molecule confined in a wedge representing the Wigner-Seitz unit cell for each morphology, ${ }^{72}$ and is the sum of the interfacial energy required to create the interface between microphase separated domains of A and B, and the stretching energy resulting from the incompressibility boundary condition for a bulk polymer. 
The shape of the wedge is determined by the mesophase, as illustrated in Figure 1.5. The height of the wedge is the radius of the round unit cell. The location of the dividing surface between microphase separated A and B domains is shown as a shaded plane. Wedge height is determined by a technique developed for polymer brushes, and is limited only by the number of chains confined to the wedge at a given surface density (chains per unit area). Chains are added incrementally until the appropriate number is present on each side of the wedge. The height of the interface determines the surface area of the interface, and is governed by the conformational properties of the constituent materials. Such round unit cell models are recognized as giving non-physical results in certain limits ${ }^{55}$ but are successful in predicting general behavior.

The stretching free energy contribution to the free energy is also determined using models developed for polymer brushes. The chain ends for each arm of the star are assumed to be found at any distance from the dividing surface within the wedge. This assumption justifies use of a quadratic chemical potential function with distance from the interface $e^{76,77}$ To calculate the stretching energy, chemical potential is integrated with respect to interface surface area.

Combining the stretching free energy component with the interfacial free energy term yields a set of equations giving free energy for the four morphologies considered, lamellae, hexagonal cylinders, BCC spheres, and the ordered, bicontinuous doublediamond (OBDD) morphology. ${ }^{44}$ These are given as equations 1,2 , and 3 ,

$$
\frac{f_{\text {lam }}}{f_{0}}=\left[\varepsilon(1-\phi)+\frac{\phi}{\varepsilon}\right]^{1 / 3}
$$




$$
\begin{gathered}
\frac{f_{c y l}}{f_{0}}=\left[\frac{2 \varepsilon \phi\left(1-\phi^{1 / 3}\right)^{3}\left(3+\phi^{1 / 2}\right)}{(1-\phi)^{2}}+\frac{2 \phi}{\varepsilon}\right]^{1 / 3} \\
\frac{f_{s p h}}{f_{0}}=\left[\frac{27 \varepsilon \phi^{4 / 3}\left(1-\phi^{1 / 3}\right)^{3}\left(\phi^{2 / 3}+3 \phi^{1 / 3}+6\right)}{10(1-\phi)^{2}}+\frac{27 \phi}{10 \varepsilon}\right]^{1 / 3}
\end{gathered}
$$

where $f$ is free energy, $f_{0}$ is the free energy of a lamellar morphology for a sample with 50 volume $\%$ of each component, $\phi$ is the volume fraction of the minority component, and $\varepsilon$ is a unified molecular asymmetry parameter describing the architectural and conformational characteristics of the molecule. An analytical solution in terms of $f$ and $\varepsilon$ for the OBDD morphology is not possible; free energy must be calculated numerically in this case.

Milner's model in fact finds the OBDD morphology to be unstable. ${ }^{11,83,84}$ Recent work by Matsen and Bates has verified this result, and attributes the instability of both the OBDD and the gyroid morphologies to packing frustration ${ }^{60,85,86}$ arising from a combination of the confinement of the block copolymer junction point to the interface between $\mathrm{A}$ and $\mathrm{B}$ domains, and the incompressibility boundary condition. The geometries of the gyroid and OBDD morphologies are such that for the polymers to fill space as required, the junction point between blocks must deviate from its preferred position at the interface. This raises the total free energy of the morphology, and makes it unstable when the penalty for this deviation is great (in the SSR). The solution for the OBDD phase in the Milner model is multiplied by a small constant prefactor ( 0.99$)$ to generate a window of stability. 
Figure 1.6 shows the morphology diagram generated using the Milner model, including the unstable OBDD phase. Morphology is given for $\mathrm{A}_{n} \mathrm{~B}_{\mathrm{m}}$ stars characterized by volume fraction, $\phi_{\mathrm{B}}$, and the unified molecular asymmetry, $\varepsilon$. The molecular asymmetry parameter, $\varepsilon$, combines architectural asymmetry, the difference in number of arms of components A and B, with conformational asymmetry, the disparity in the spacefilling characteristics of components A and B. The molecular asymmetry parameter is given as

$$
\varepsilon=\left(\frac{n_{A}}{n_{B}}\right) \cdot\left(\frac{l_{A}}{l_{B}}\right)^{1 / 2}=\left(\frac{n_{A}}{n_{B}}\right) \cdot\left(\frac{\frac{V_{A}}{R_{A}^{2}}}{\frac{V_{B}}{R_{B}{ }^{2}}}\right)^{1 / 2}=\left(\frac{n_{A}}{n_{B}}\right) \cdot\left(\frac{\frac{v_{A}}{b_{A}^{2}}}{\frac{v_{B}}{b_{B}{ }^{2}}}\right)^{1 / 2}
$$

where $n_{\mathrm{i}}$ is the number of arms of component $i$, and $l_{\mathrm{i}}$ is a material parameter describing the conformational behavior of component $i$. This material parameter is defined as $l_{\mathrm{i}}=$ $\mathrm{V}_{\mathrm{i}} /\left(\mathrm{R}_{\mathrm{i}}{ }^{2}\right)$, where $\mathrm{V}_{\mathrm{i}}$ is the volume of component $i$ having radius of gyration, $\mathrm{R}_{\mathrm{i}}$. Both terms are proportional to chain length, $N$, and the equation defining $\varepsilon$ reduces to a function of arm number, $\mathrm{n}_{\mathrm{i}}$, segmental volume, $v_{\mathrm{i}}$, and statistical segment length, $b_{\mathrm{i}}$.

The model predicts that the volume fraction range in which a given morphology is predicted to be observed shift to higher volume fraction with increasing molecular asymmetry. This arises from the effect of confining multiple arms to one side of an interface. To illustrate, consider a conformationally symmetric linear diblock of equal volume fractions $\mathrm{A}$ and $\mathrm{B}\left(\phi_{\mathrm{B}}=0.5\right)$, such as illustrated in Figure 1.7a. If the single arm of $A$ is then replaced by two arms of $A$ exactly $1 / 2$ the length of the original arm of $A$, to maintain a flat interface (lamellar morphology), the two arms must be stretched more 
than the single arm of $\mathrm{A}$ in the $\mathrm{AB}$ diblock (Figure 1.7b). This results in an increase in free energy, which may be partially alleviated by curvature of the interface away from the two A arms (Figure 1.7c). Volume fraction remains unchanged, but morphology has been dramatically altered by architecture. This effect produces the shift to higher volume fractions, with increasing molecular asymmetry, $\varepsilon$, of the volume fraction ranges in which block copolymer morphologies are predicted. As illustrated in Figure 1.6, for a series of $\mathrm{A}_{n} \mathrm{~B}$ stars, as $n$ increases the change in predicted morphological behavior is significant. 


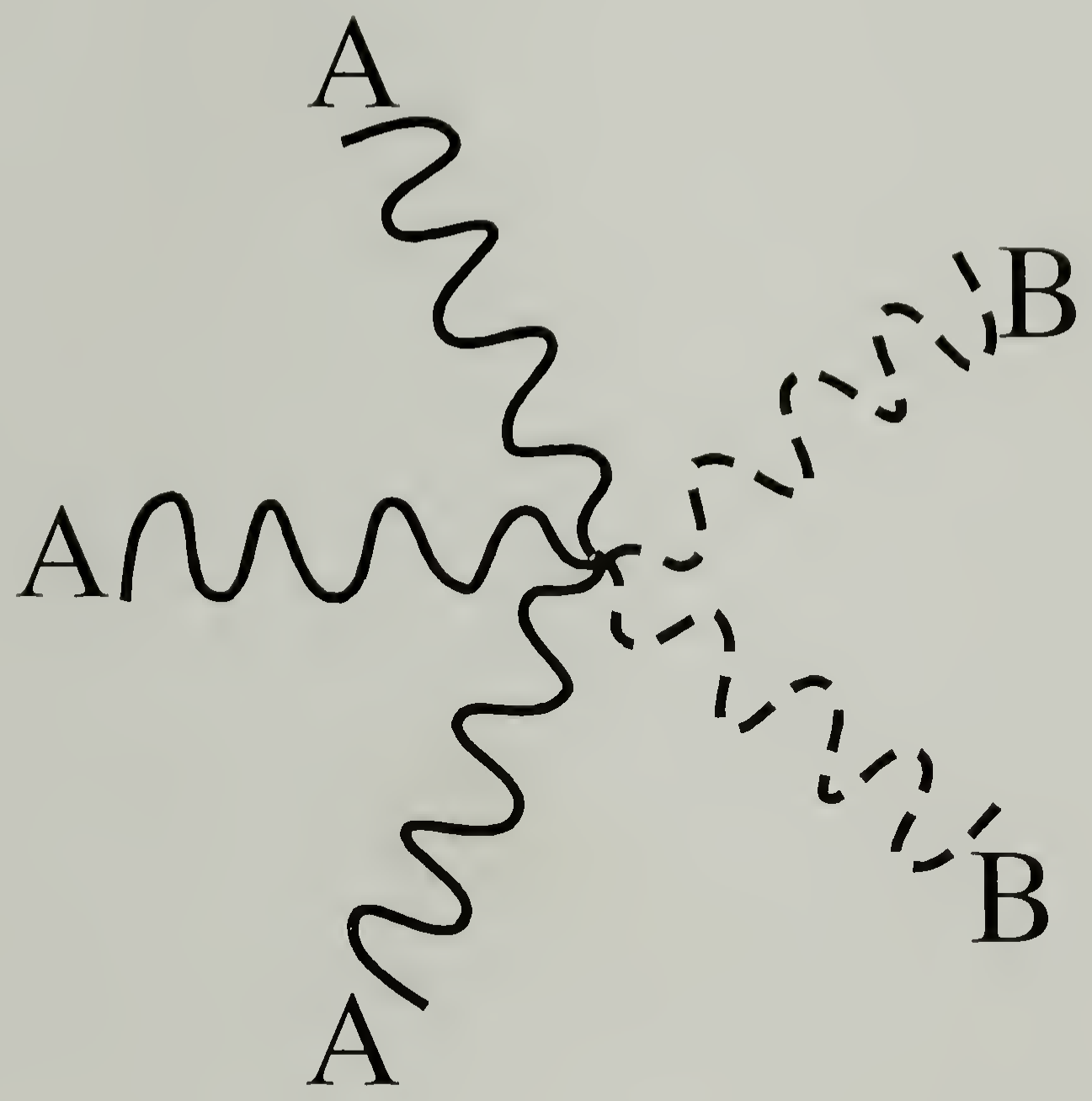

Figure 1.1. Schematic of an $\mathrm{A}_{3} \mathrm{~B}_{2}$ miktoarm star comprised of three arms of polymer $A$ and two arms of polymer $B$. 


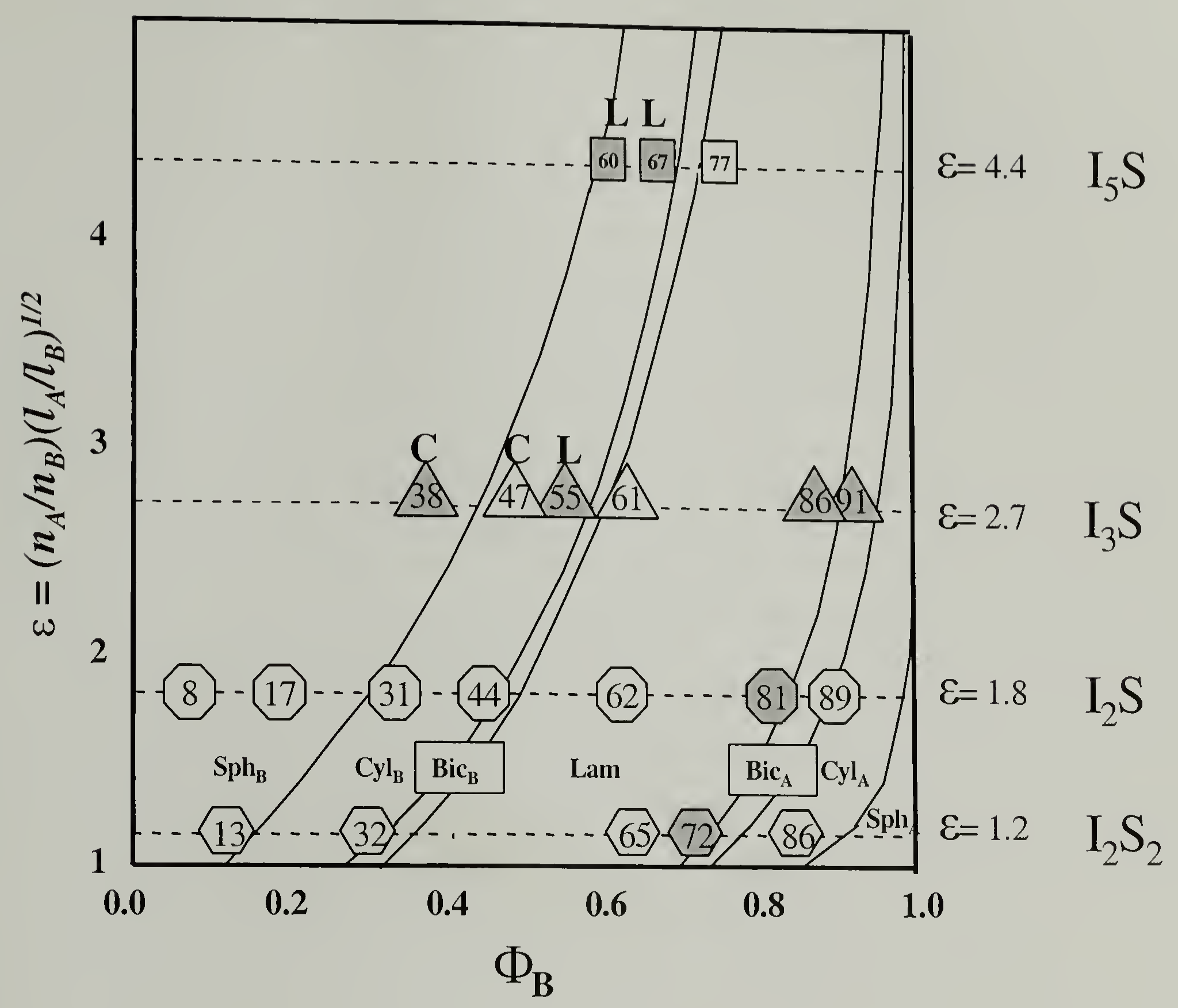

Figure 1.2. Strong segregation regime morphology diagram generated using the Milner model. Miktoarm star morphology is given as a function of volume fraction, $\phi_{\mathrm{B}}$, and molecular asymmetry. Shaded symbols indicate a sample whose morphology disagrees with that predicted by theory. The numbers in the symbols are the volume fraction of these samples. (For $\mathrm{I}_{2} \mathrm{~S}, \mathrm{I}_{3} \mathrm{~S}, \mathrm{I}_{5} \mathrm{~S}$, these numbers are PS volume percentage, for $\mathrm{I}_{2} \mathrm{~S}_{2}$ samples, the numbers are PI volume percentage). 


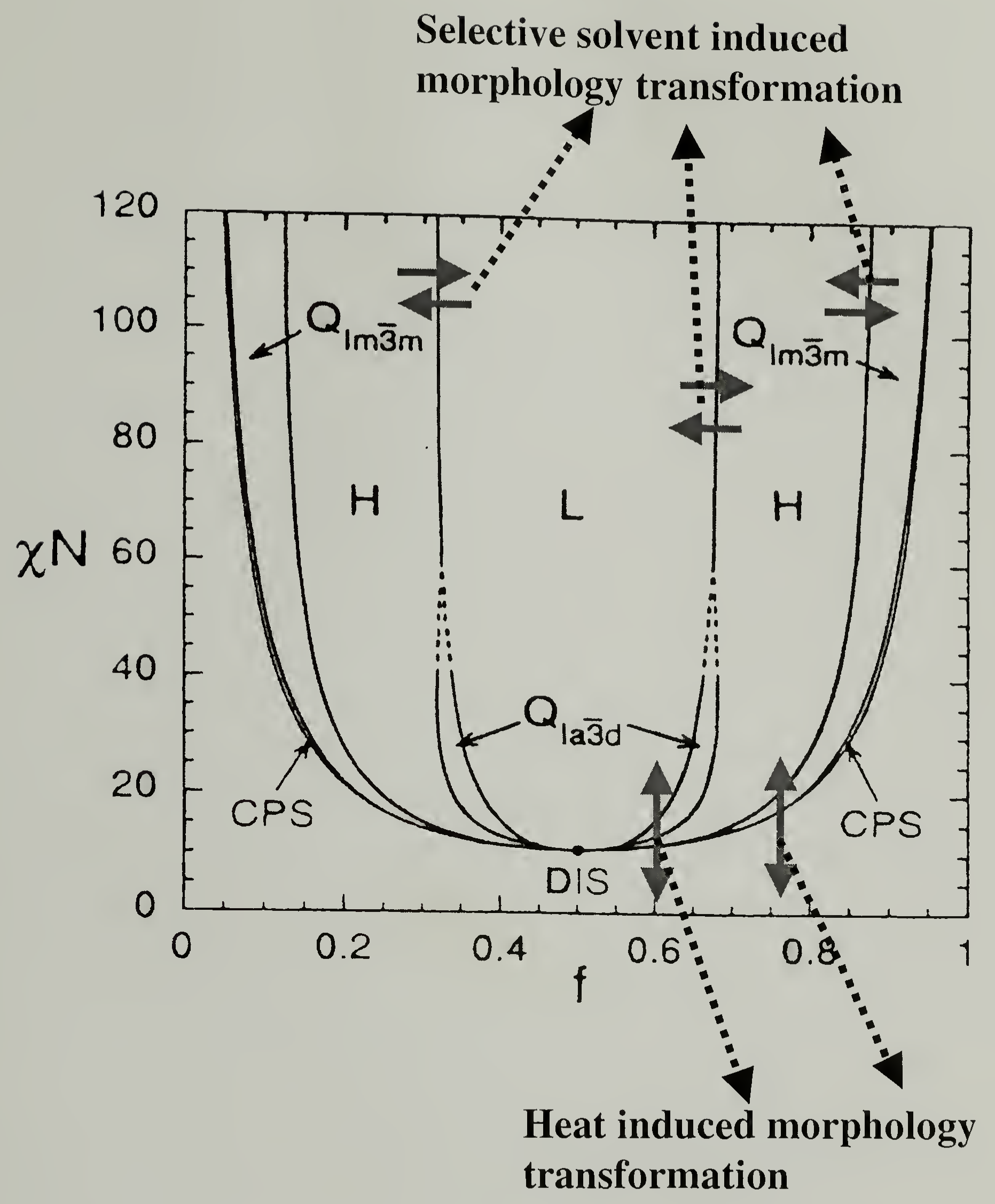

Figure 1.3. Schematics of morphology transformations. The vertical arrows indicate the schematics of reversible heat induced morphology transformation; well the horizontal arrows indicate the schematics of selective solvent induced irreversible morphology transformation. 


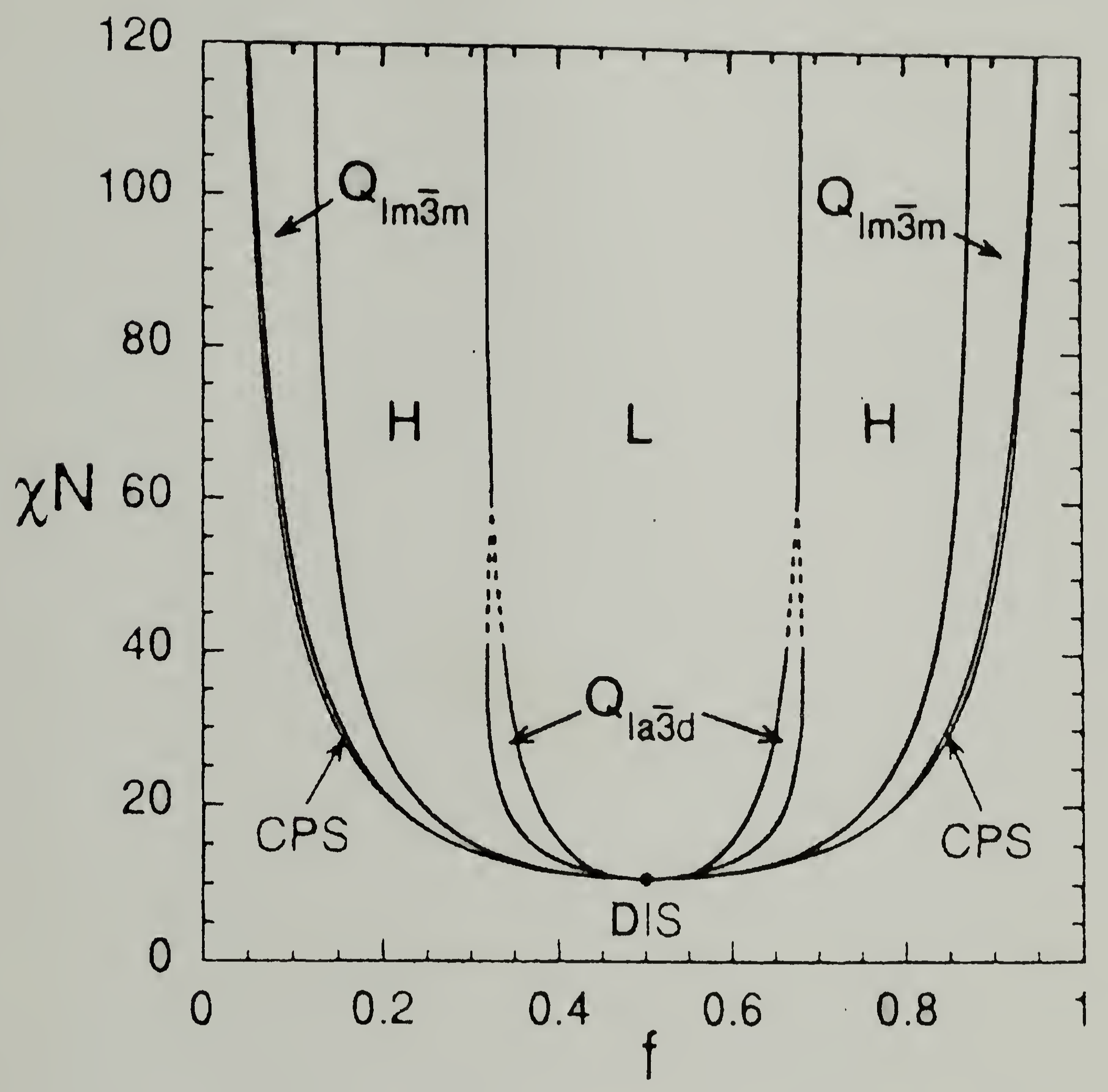

Figure 1.4. Morphology diagram for a linear $\mathrm{AB}$ diblock copolymer calculated by Matsen and Bates, spanning from the order-disorder transition into the strong segregation regime. 

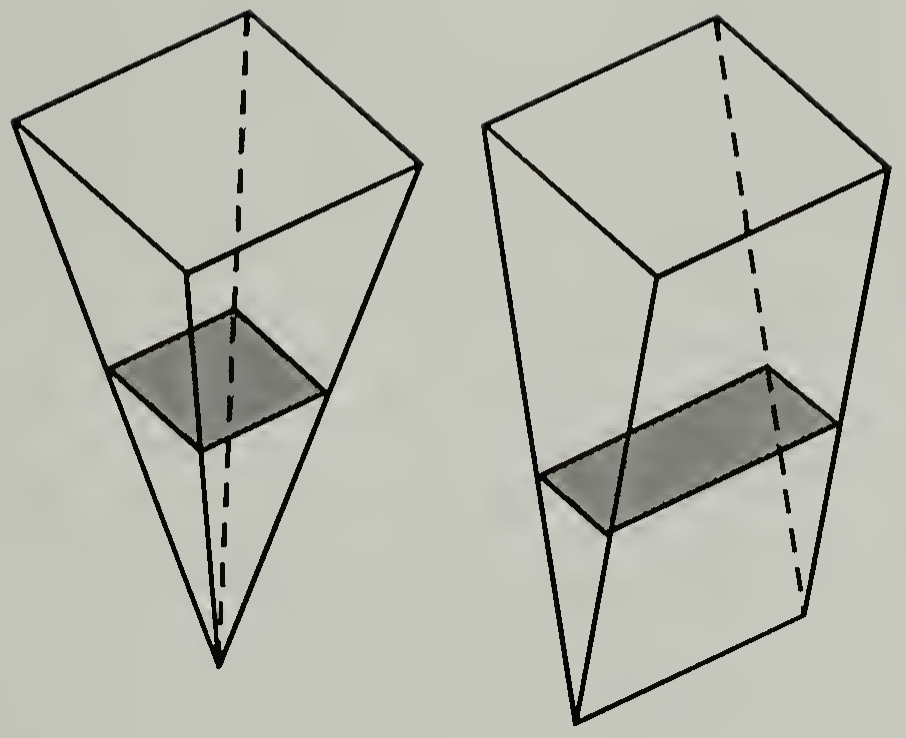

Sphere

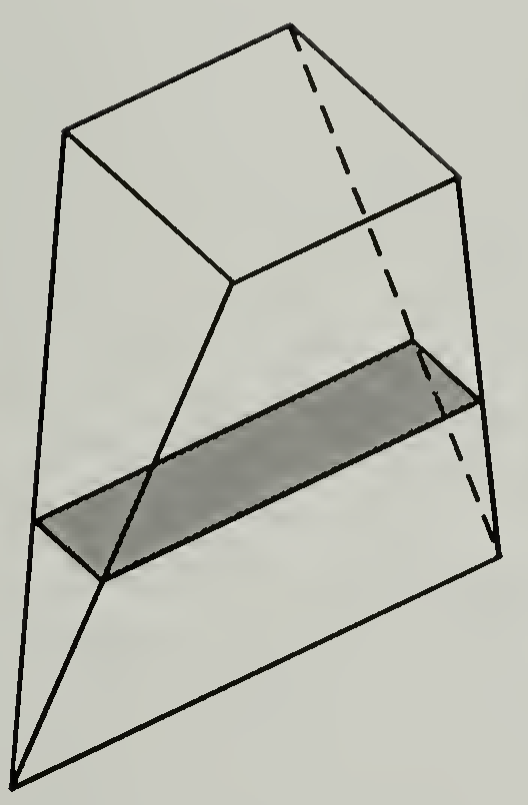

Bicontinuous

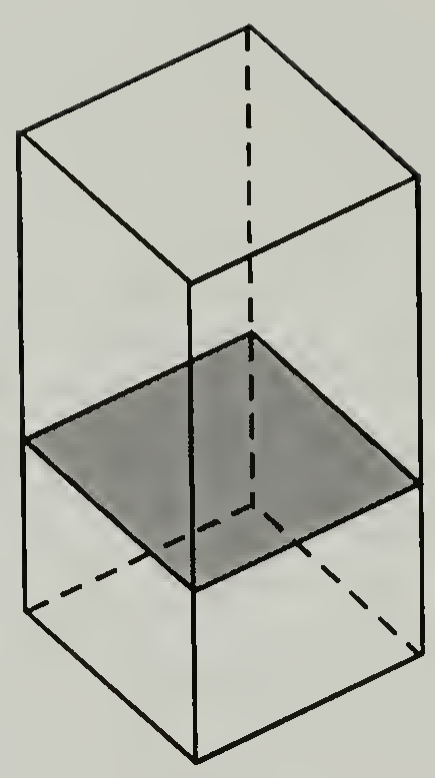

Lamella

Figure 1.5. Gaussian wedges for lamellar, cylindrical, spherical, and OBDD bicontinuous morphologies used in the Milner model for calculating morphological behavior of miktoarm star block copolymers. The shaded planes represent the interface between microphase-separated domains of components $\mathrm{A}$ and $\mathrm{B}$. 


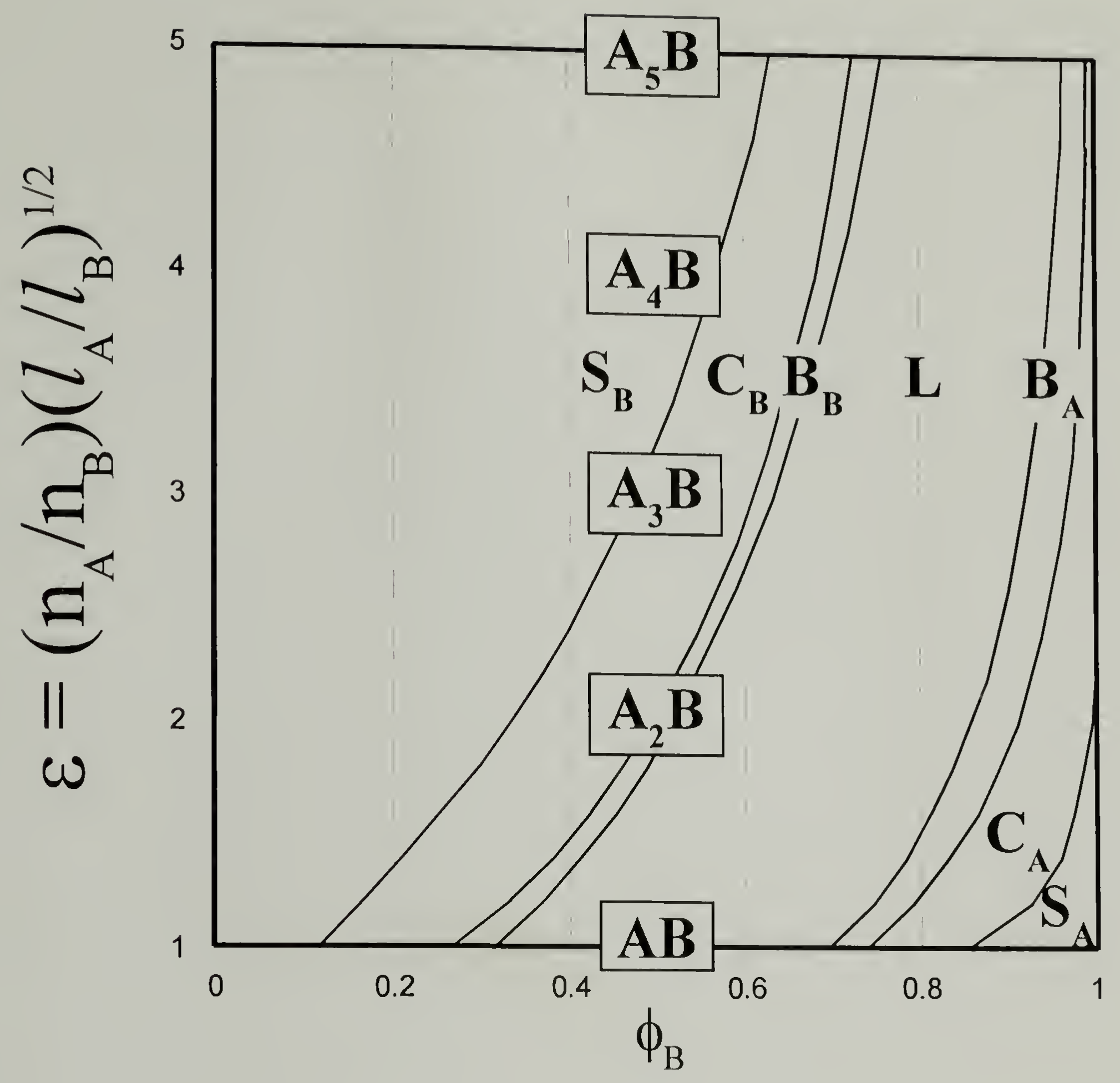

Figure 1.6. Strong segregation regime morphology diagram generated using the Milner model. Miktoarm star morphology is given as a function of volume fraction, $\phi_{\mathrm{B}}$, and molecular asymmetry. Boxes with $\mathrm{A}_{n} \mathrm{~B}$ stars indicate the level of molecular asymmetry for conformationally symmetric $A_{n} B$ miktoarm stars, illustrating the effect of architectural asymmetry on morphological behavior. 


\section{CHAPTER 2}

\section{MORPHOLOGICAL BEHAVIOR OF I $\mathrm{I}_{2}$ S SINGLE GRAFT BLOCK COPOLYMER / HOMOPOLYMER BLENDS}

\subsection{Abstract}

This work is part of an extensive study of model nonlinear block copolymer / homopolymer blends. Effects of graft molecular architecture on the morphology of block copolymer / homopolymer blends have been examined. The single graft $Y$ shaped block copolymers used in the study are $\mathrm{I}_{2} \mathrm{~S}$ block copolymers, which have two low polydispersity (PDI) polyisoprene arms and one low PDI polystyrene arm joint at a single junction point. Previously reported linear diblock copolymer / homopolymer blend systems showed that the order-order transitions (OOTs) occur at about the same volume fractions as in pure linear diblock copolymers. The OOT occurs at the same volume fraction regardless of the direction from which it is approached, i.e. blending homopolymer A with a diblock which forms A cylinders in a B matrix to push it toward lamella or blending B homopolymer with a lamellar diblock to push it back toward cylinders. ${ }^{17,18,20}$ This study shows that when blending a homopolymer with an $\mathrm{I}_{2} \mathrm{~S}$ block copolymer that the OOTs split so that they occur at different volume fractions depending up whether they are approached by blending homopolymer into the two arm or the one arm side of the block copolymer interface. A perforated lamellar morphology is obtained in a blend of homopolystyrene (hPS) and a lamella forming single graft block copolymer, 
and it is found to be stable to thermal annealing. TEM features of perforated lamella are analyzed.

\subsection{Introduction}

Recently, there have been many investigations concerning the effects of graft block copolymer molecular architecture on morphology using well defined, model branched block copolymer materials. ${ }^{2-5,8,10,87-89}$ The present study investigates the effect for molecular asymmetry and graft architecture on blend morphology. The morphologies of neat microphase-separated $\mathrm{A}_{2} \mathrm{~B}$ single graft copolymers have been studied by Pochan, Gido et. al. ${ }^{2,87}$ The results of the experimental study were plotted on a theoretical morphology diagram for asymmetric miktoarm stars, calculated by Milner. ${ }^{11}$ For architecturally and conformationally asymmetric miktoarm stars of type $A_{n} B_{m}$, this theory predicts morphology as a function of $\mathrm{B}$ component volume fraction, $\phi_{\mathrm{B}}$, and a molecular asymmetry parameter, $\varepsilon=\left(n_{\mathrm{A}} / n_{\mathrm{B}}\right)\left(l_{\mathrm{A}} / l_{\mathrm{B}}\right)^{1 / 2}$. Here, $n_{\mathrm{A}}$ and $n_{\mathrm{B}}$ are the numbers of arms of block materials $\mathrm{A}$ and $\mathrm{B}$, and $l_{\mathrm{i}}=\left(\mathrm{V}_{\mathrm{i}} / \mathrm{R}_{\mathrm{i}}{ }^{2}\right)=v_{\mathrm{i}} / \mathrm{b}_{\mathrm{i}}{ }^{2} . \quad \mathrm{V}_{\mathrm{i}}$ and $\mathrm{R}_{\mathrm{i}}$ are volume and radius of gyration of one arm of polymer $i$, while $v_{\mathrm{i}}$ is the segmental volume and $\mathrm{b}_{\mathrm{i}}$ the statistical segment length of component $i$. The morphologies observed for the $\mathrm{A}_{2} \mathrm{~B}$ materials showed general agreement with the shift in composition ranges predicted by the theory. However, for some samples, the Milner calculation slightly overestimated the degree of shift in the order-order transitions. ${ }^{87}$ Some other studies on $\mathrm{S}_{2} \mathrm{I}, \mathrm{I}_{2} \mathrm{~S}$ and $\mathrm{I}_{3} \mathrm{~S}$ also showed qualitative agreement but quantitative disagreement. ${ }^{7-10,89,90}$ The Milner diagram is calculated for pure block copolymer. In this study, we will try to use it as a guide to 
understand the effects of homopolymer blending on the morphological transitions of $\mathrm{I}_{2} \mathrm{~S}$ block copolymer systems.

Linear diblock copolymer / homopolymer blends and linear multi block copolymer / homopolymer blends systems have been extensively studied. ${ }^{13-25}$ Previously reported linear diblock copolymer / homopolymer blend results showed that the OOTs in the blends occur at about the same volume fractions as those of pure linear diblock copolymers. ${ }^{17,18,20}$ However, for architecturally asymmetric graft copolymers, blending homopolymer into the one arm side or into the multi-arm side of the interface may produce different morphological results. In the present study, $\mathrm{I}_{2} \mathrm{~S}$ block copolymers are blended with homopolymers of polystyrene (hPS) or homopolymer polyisoprene (hPI). The schematic of an $\mathrm{I}_{2} \mathrm{~S}$ single graft block architecture with one PS arms and two PI arms is shown in Figure 2.1.

One of the blends in this study is observed to form a hexagonally perforated lamellar structure. Hexagonally perforated lamellae, which resemble the cantenoidlamellar structure derived by periodic area-minimizing surfaces calculations, ${ }^{48}$ were also observed recently in linear diblock systems ${ }^{35,37-39,46,50-52}$ in a narrow volume fraction region between HEX and LAM. Several linear block copolymer / homopolymer blends have been previously shown to form perforated lamellar structures. ${ }^{22,53}$ To date, however, ordered perforated lamellar structures have not been observed in block copolymers or blends involving block copolymers with graft architectures. 
Controversy exists regarding the details of the perforated lamella structure and the stability of the morphology. Both ABC stacking of the hexagonally perforated layers ${ }^{38,52}$ and a combination of an $\mathrm{ABC}$ and $\mathrm{AB}$ stacking ${ }^{39}$ were suggested for the perforated lamellar morphology. Using the methods of Semenov ${ }^{55}$ to estimate the free energy of perforated lamella in the strong segregation limit (SSL), Fredrickson ${ }^{56}$ showed that the perforated lamella is metastable with respect of cylinders and lamella at $\phi=0.35$. Qi and Wang ${ }^{57.59}$ showed that the perforated lamellae appear as a "pseudostable" morphology during the lamellar to cylinder transition.

\subsection{Experimental}

The synthesis of the $I_{2} S$ block copolymers and the subsequent molecular characterization was described in a previous publication. ${ }^{87}$ The materials were produced by anionic polymerization under high vacuum in all glass reactors. The graft architecture was generated using coupling with trichloromethylsilane. The volume fraction and morphology of the neat $\mathrm{I}_{2} \mathrm{~S}$ block copolymers and homopolymers used in this study are listed in Table 2.1. The polystyrene of both the $\mathrm{I}_{2} \mathrm{~S}$ block copolymers and the homopolymer were deuterated. We use low molecular weight (MW) homopolymer in all the blend systems so that the distribution of the homopolymer within the block copolymer domains of like material is relatively uniform. ${ }^{14,19,91}$ This study utilizes blends which have overall high PS composition. Blends with $\mathrm{I}_{2} \mathrm{~S}$ block copolymers having high overall PI composition have been examined separately. ${ }^{92}$ 
Graft copolymer and homopolymer in the proportions to give the desired blend were co-dissolved in toluene, a non-preferential solvent. Solid films approximately $1 \mathrm{~mm}$ thick were slowly cast from these co-solutions at room temperature over the course of 10 to 14 days. Residual toluene was removed by placing the samples under vacuum at ambient temperature for another week. Each sample was thermally annealed in a vacuum oven at $120^{\circ} \mathrm{C}$ for two weeks to further promote the approach to equilibrium. The samples were then cooled under vacuum to room temperature over a period of several hours.

Sample morphologies were characterized using a combination of transmission electron microscopy (TEM) and small-angle X-ray scattering (SAXS). To prepare thin sections for microscopy, a Leica Ultracut UCT microtome equipped with a Leica EM FCS cryogenic sample chamber operated at $-110^{\circ} \mathrm{C}$ was used to cut sections approximately $500 \AA$ in thickness. The sections were collected on TEM grids and stained for four hours in $\mathrm{OsO}_{4}$ vapor. The $\mathrm{PI}$ microdomains are preferentially stained by $\mathrm{OsO}_{4}$, rendering them dark relative to PS microdomains due to mass-thickness contrast in the TEM. A JEOL 100CX TEM, operated at an accelerating voltage of $100 \mathrm{kV}$, was used to image the stained sections. SAXS data were collected at the Advanced Polymers Beamline (X27C), located at the National Synchrotron Light Source at Brookhaven National Labs (BNL), Upton, NY. Two-dimensional scattering patterns were collected on Fujitsu image plates, and then read by a Fujitsu BAS 2000 image plate reader. Custom software at BNL was used to subtract background noise and perform circular averaging. Data were collected for a wavelength of $1.307 \AA$ and a camera length of 1410 $\mathrm{mm}$. 
Four different series of blends are examined. Blends are designated as either IYn$\mathrm{m}$ or SYn-m. The first character, I or S, indicates that the homopolymer used in the blend series is either homopolyisoprene (I) or homopolystyrene (S). The first number $n$ indicates the PS volume fraction of the Y shaped graft copolymer $\left(\mathrm{I}_{2} \mathrm{~S}-89, \mathrm{I}_{2} \mathrm{~S}-81\right.$, or $\mathrm{I}_{2} \mathrm{~S}$ 62 from reference 4) on which the blend series is based. The last number, m, indicates the PS volume fraction of the overall blend after addition of homopolymer. The molecular characteristics of the block copolymers and homopolymers are listed in Table 2.1. The compositions of all the blends studied in this paper are given in Table 2.2.

\subsection{Results}

\section{SY62 series}

The pure single graft block copolymer $\mathrm{I}_{2} \mathrm{~S}-62$ with polystyrene volume fraction $\phi_{\mathrm{PS}}=62 \%$ formed a lamella morphology with a long period of $39.0 \pm 0.5 \mathrm{~nm} .{ }^{87}$ The SAXS data for the SY62 blend series is shown in Figure 2.2. The first three blends, SY62-73, SY62-76 and SY62-79, maintain the lamellar morphology with slightly increasing lamellar spacing (Table 2.2). The fourth blend, SY62-82, forms perforated lamella. The layer spacing of perforated layers is $37.5 \mathrm{~nm}$, which is a decrease from the lamellar spacing of SY62-79 due to the morphology change. The $\sqrt{3} \mathrm{q}^{*}$ reflection results from the ordering of the perforations. ${ }^{39,52}$ This scattering data does not permit the determination of detailed structural information about the stacking of the perforated layers. The perforated lamellar morphology persisted upon annealing at $120^{\circ} \mathrm{C}$ for 2 
weeks. The fact that a perforated lamellar structure is found at this unusual composition is a direct result of the $\mathrm{I}_{2} \mathrm{~S}$ molecular architecture of the block copolymer used in the blend. TEM micrograph of the blend series is shown in Figure 2.3.

As the PS volume fraction further increases, the blends SY62-85 and SY62-89 both form cylindrical morphologies, and the cylinder (100) d-spacing increases as the homopolymer volume fraction increases (Table 2.2). TEM observation (data not shown) confirmed the cylindrical morphologies of SY62-85 and SY62-89. The morphologies of the SY62 blends series are mapped onto the theoretical morphology diagram in Figure 2.4 .

\section{SY81 series}

The pure single graft block copolymer $I_{2} S-81$ with polystyrene volume fraction $\phi_{\mathrm{PS}}=81 \%$ formed a regular cylinder morphology with reported cylinder 100 spacing of $31.0 \pm 0.5 \mathrm{~nm} .{ }^{87}$ The two blend samples of the SY81 series were made by blending low molecular weight homopolystyrene with $\mathrm{I}_{2} \mathrm{~S}-81$. The morphologies of the SY81 blend series are mapped onto the morphology diagram in Figure 2.5. TEM images of SY81-82 and SY81-85 are shown in Figure 2.6. The long-range order of the morphology decreases as hPS concentration increases and essentially vanishes for SY81-85. The SAXS data shown in Figure 2.7 support this conclusion. The (100) spacings of SY81-82 and SY81-85, $31.6 \mathrm{~nm}$ and $30.6 \mathrm{~nm}$ respectively, are nearly the as same as that of pure $\mathrm{I}_{2} \mathrm{~S}-81$. The secondary peak of SY81-85 is broad and weak. We calculated form factor scattering that results from disordered arrangements of domains of spheres and cylinders. Domain sizes are obtained by using the primary peak to get an average center-to-center 
distance between neighboring domains. This spacing along with the known PS and PI volume fraction can be used to calculate sphere and cylinder radii provided that a model for how the domains fill space is assumed. For the purposes of these calculations cylinder radii were obtained assuming a hexagonal packing and sphere radii were determined using both BCC and SC lattice packings. ${ }^{93,94}$ All the form factors for cylinders and spheres (both BCC and SC) fit the data poorly. This suggests that the broad peak is actually from weak and diffuse $\sqrt{3} q^{*}$ and $2 q^{*}$ Bragg reflections, and the sample has a poorly ordered cylindrical morphology. Both TEM and SAXS indicate that the long-range order of the cylinder morphology decreases as more PS homopolymer is added.

\section{IY8I series}

The morphologies of the IY81 blend scries are also mapped onto the morphology diagram in Figure 2.5. In this series, in which hPI is blended into $\mathrm{I}_{2} \mathrm{~S}-81$, samples IY8180, IY81-76, IY81-73, IY81-71 and IY81-66 all form cylindrical morphologies. Figure 2.8a shows a TEM image of one of these cylindrical morphologies (IY81-66), the others are similar. There is little change in long-range order with increasing hPI concentration among these cylindrical samples but all are quite well ordered as compared to the SY81 series. With only one volume percent more PI, blend IY81-65 changed to the lamellar morphology. No intermediate morphology, such as gyroid or perforated lamella, was observed between lamellac and cylinders in this series. Figure 2.9 shows the SAXS data for this series. The cylinder (100) spacings, shown in Table 2.2, increased with increasing amounts of homopolyisoprene in the blends. 


\section{IY89 series}

The pure single graft block copolymer $\mathrm{I}_{2} \mathrm{~S}-89$, with $\phi_{\mathrm{PS}}=0.89$, formed a morphology of disordered, worm-like cylinder domains of PI in a matrix of PS. ${ }^{2,87}$ TEM images of the IY89 series, which are produced by blending increasing amounts of hPI with $\mathrm{I}_{2} \mathrm{~S}-89$, are shown in Figure 2.10. Figure 2.10b reveals that IY89-86 formed a disordered worm-like cylinder morphology, similar to pure $\mathrm{I}_{2} \mathrm{~S}-89$ (Figure 2.10a). Figure $2.10 \mathrm{~b}$ is a TEM image of blend IY89-82 showing that the dark stained PI domains form ring-like structures and short line segments. Blends IY89-80, IY89-77, and IY89-71 with increasing PI volume fractions also formed sheet-like morphologies but in these cases closed vesicles were not favored (although a few isolated vesicles can be observed in the TEM images). The predominant structures were continuous, convoluted sheets which extend over great distances relative to their sheet thickness. A TEM image of this structure in IY89-80 and IY89-71 are shown in Figure 2.10d and Figure 2.10e. Due to the lack of lattice order in the IY89 blend series, SAXS data was not used for structural investigation. Figure 2.11 shows a higher magnification TEM tilt series of blend IY8982 that reveals that some PI domains which appear as short line segments, are actually pieces of sheets. Some PI domains which appear as rings are actually sections through vesicles, and other objects seem to be caps sectioned off the top or bottom of vesicles. The schematic of the vesicle and their TEM projection are illustrated in Figure 2.12. The rings and caps have different projections with the change of viewing angles and microtome positions. The thickness of these PI sheets is about $12 \mathrm{~nm}$. The diameters of the vesicles as well as the lengths of the PI sheets are about 150 500nm. The morphology of IY89-82 is composed of bilayer sheets which tend to form closed vesicies or isolated sheets which seem to be limited in there lateral extent to an upper limit of 
about 40 times the PI layer thickness. The vesicle and sheet structures of the IY89 series are unusual because the spaces inside the vesicles and between vesicles and/or sheets are frequently much larger (up to almost $1 \mu \mathrm{m}$ in size) than the radii of gyration of the PS blocks of the $\mathrm{I}_{2} \mathrm{~S}-89$ block copolymer (PS molecular weight of $87,300 \mathrm{~g} / \mathrm{mol}$ ). The TEM images suggest that the vesicles and sheets grow by formation of organized block copolymer bilayers in a matrix of un-microphase separated material.

\subsection{Discussion}

For the lamella samples in the SY62 series, the polystyrene brush height and the polyisoprene brush height can be calculated by $h_{P S}=(1 / 2) d_{l a m} * \phi_{P S}$ and $h_{P I}=(1 / 2) d_{\text {lam }}{ }^{*} \phi_{P S}$ respectively, where $d_{\text {lam }}$ is the long period of the lamella structure. The area per block copolymer chain junction can be calculated by $A=M_{P I} /\left(d_{l a m}{ }^{*} \phi_{P I} * \rho_{P I} * N_{a v}\right)$, where $M_{P I}$ is the average total molecular weight of the two PI arms of each block copolymer molecule, $\rho_{\mathrm{Pl}}$ is the density of polyisoprene and $\mathrm{N}_{\mathrm{av}}$ is Avagadro's number. After blending in homopolymer, the PS domains swell both parallel and perpendicular to the interface. We can calculate the relative vertical expansion of the PS domain in the blends with respect to the PS brush height of the pure block copolymer $\left(I_{2} S-62\right): \lambda^{\perp}=h / h_{0}$ where $h_{0}$ is the PS brush height of the pure block copolymer $\mathrm{I}_{2} \mathrm{~S}-62$, and $\mathrm{h}$ is the PS brush height of the blend sample. From the area per junction of each blend, the relative expansion ratio in the direction parallel to the interface is calculated by $\lambda^{\prime \prime}=\left(\mathrm{A} / \mathrm{A}_{0}\right)^{0.5}$. Therefore, the total swelling of the PS domains can by expressed by the two expansion ratios: $V / V_{0}=\lambda^{\perp}\left(\lambda^{\prime \prime}\right)^{2}$. If the domain expansion is uniform in all directions, $V / \mathrm{V}_{0}=\lambda^{3}$, with $\lambda=\lambda^{\perp}=\lambda^{\prime \prime}$; if the 
expansion is anisotripic then $\lambda^{\perp}$ and $\lambda^{\prime \prime}$ will not be equal and we define their ratio as the expansion asymmetry coefficient: $\mathrm{K}=\lambda^{\perp} / \lambda^{\prime \prime}$. All the calculated results are listed in Table 2.3 and the asymmetry coefficient for lamella forming SY62 series blends verses homopolymer volume fraction is plotted in Figure 2.13, from which it is apparent that $\mathrm{K} \approx 1$ and changes little with increasing hPS concentration.

The cylinder core radii $\mathrm{R}$ of the samples in the IY81 and SY81 blend series were calculated from the (100) interplanar spacing determined by SAXS and the volume fraction of PI $\left(\mathrm{R}_{\mathrm{cyl}}=\mathrm{d}_{\text {nex }(100)}\left(\frac{\phi_{\mathrm{Pl}}}{\pi} \operatorname{Sin} \frac{\pi}{3}\right)^{\frac{1}{2}}\right)$. The area per block copolymer chain junction can be calculated by $A=\left(2 M_{P I} /\left(\rho_{P I} R_{c y l} N_{a v}\right)\right.$. For the IY81 series, $M_{P I}$ is the effective total polyisoprene molecular weight per junction point, which includes the PI content of a single block copolymer molecule as well as the total amount of hPI per block copolymer molecule. This is calculated by dividing the total number of hPI molecules in the system by the total number of block copolymer molecules. We use the cylinder core radius $\mathrm{R}_{\text {cyl }}$ as the average PI brush height; the polystyrene brush height can be calculated by hps $=\left(\sqrt{3} d_{\text {hex(100) }}^{2} / \pi\right)^{1 / 2}-R_{\text {cyl }}$. From these values, $\lambda^{\prime \prime}, \lambda^{\perp}$ and $K$ are calculated for the IY81 and the SY81 series and are listed in Table 2.3. The asymmetric swelling coefficient $\mathrm{K}$ is plotted verses homopolymer volume fraction in Figure 2.13 for cylinder forming blends in both the SY81 and the IY81 series.

Figure 2.13 shows that expansion coefficients K of the SY62 series and the SY81 series are close to one and change little with increasing $\phi_{\mathrm{PS}}$. Thus $\lambda^{\perp} \approx \lambda^{\prime \prime}$, i.e. in both 
series, the homopolystyrene swells the PS domains homogeneously. Because the PS and PI domains share the same interface, the component of PS domain swelling parallel to the interface in the SY81 series leads, through conservation of volume, to a decrease of the brush height in the PI domains. Consequently, the overall structural periodicity, the sum of the PS and PI domain thickness, changes very little (Table 2.2). This behavior is considerably different from the IY81 series in which swelling perpendicular to the interface is favored. This can be seen in Figure 2.13 where $\mathrm{K}$ increases with increasing hPI content. As hPI is added, the PI layer thickness increases much more strongly than the interfacial area per junction. In this case the overall structural periodicity was found to increase rather strongly with the addition of hPI as indicated in Table 2.2.

Previously published studies on the swelling behavior of block copolymer / homopolymer blends, indicate that the degree to which homopolymer penetrates into the brush of the corresponding block of the copolymer depends on the ratio, $\alpha$, of the homopolymer molecular weight to the molecular weight of the block of the same type $^{14,19,91,95}$ In both the SY62 and SY81 series $\alpha=0.01$, and in the IY81 series $\alpha=0.09$. Therefore, a comparison of swelling behavior on the two arm PI side of the interface to that on the one arm PS side of the interface is potentially complicated by a difference in $\alpha$. However, comparison to data of Winey, Thomas and Fetters ${ }^{19}$ on blends of linear diblocks with homopolymer, also plotted in Figure 2.13, suggest that the molecular architecture effect on $\mathrm{I}_{2} \mathrm{~S}$ containing blends is significant enough to be distinguishable even with the $\alpha$ difference. 
The linear diblock swelling data in Figure 2.13 indicates that with $\alpha=0.1,2600$ $\mathrm{g} / \mathrm{mol}$ hPS swells the PS domain of PS-b-PI (PS 27,000 g/mol, PI 22,000 $\mathrm{g} / \mathrm{mol}$ ) relatively homogeneously. The SY62 and SY81 series, with $\alpha=0.01$, both show similarly isotropic swelling behavior. This observation is consistent with theoretical calculations of Matsen 95 and Shull and Winey ${ }^{91}$, which indicate that at $\alpha$ below 0.125 to 0.10 that homopolymer homogeneously swells the like block of a linear diblock. This suggests that in the IY81 blend series with $\alpha=0.09$ that the effect of miktoarm star architecture plays a key role in the asymmetric swelling behavior. At the very least, the fact that there are two PI blocks per molecule in the PI block copolymer brush, reduces the value of $\alpha$ for which swelling becomes homogenous to something smaller than the value of 0.1 found for linear diblock containing blends. The IY81 series and the Winey $2600 \mathrm{~g} / \mathrm{mol}$ hPS / linear diblock blend series have similar $\alpha$ values of 0.09 and 0.1 respectively. However, the IY81 series in which the homopolymer is blended into the two arm side of the block copolymer interface shows dramatic swelling anisotropy, while the linear diblock containing blend series shows isotropic behavior.

The miktoarm star molecular architecture of the block copolymer contributes to the differences in homopolymer swelling behavior on the two sides of the interface between the SY81 and SY62 series, and the IY81 series. The higher crowding of the grafted block copolymer brush on the PI side of the interface, due to the fact that there are two PI blocks per $\mathrm{I}_{2} \mathrm{~S}$ molecule, ${ }^{10,96}$ may make it more difficult for the hPI to penetrate into the PI brush than for the hPS to penetrate the PS brush on the other side of the interface. In the IY81 series, this results in more hPI adding to the center of the PI 
domains rather than penetrating to the interface, thus increasing the domain thickness faster than the interfacial area as more homopolymer is added. On the other side of the interface, with one PS arm per block copolymer molecule, the hPS in the SY81 and SY62 series penetrates more easily into the brush resulting in more isotropic swelling behavior.

In diblock copolymer / low molecular weight homopolymer blends, the OOTs generally occur at the about the same overall volume fractions as those of pure diblock copolymer. ${ }^{17,18,20}$ Additionally, an OOT occurs at the same volume fraction regardless of the direction from which it is approached, i.e. blending homopolymer A with a diblock that forms A cylinders in a B matrix to push it toward lamella, or blending B homopolymer with a lamellar diblock to push it back toward A cylinders. ${ }^{17,18,20}$ In the present study, blending homopolymer into each sides of the interface in systems based on the asymmetric $\mathrm{I}_{2} \mathrm{~S}$ molecular architecture results in a splitting of the OOT location such that it occurs at different volume fractions depending on the direction from which it is approached. In the IY81 series, blending hPI with cylinder forming $\mathrm{I}_{2} \mathrm{~S}-81$ results in a transition to lamella at $\phi_{\mathrm{PS}}=0.65$ to 0.66 . This is a substantial shift from the cylinder to lamellar transition predicted by the Milner morphology diagram (around $\phi_{\mathrm{PS}} \approx 0.80$ ), and is close to the PS volume fraction at the lamellar-cylinder OOT in linear diblocks. Figure 2.4 indicates that the OOTs of the SY62 series, in which a lamellar system is pushed toward cylinders by blending with hPS, occur at a $\phi_{\mathrm{PS}}$ value closer to that predicted by the Milner theory for architecturally asymmetric $\mathrm{I}_{2} \mathrm{~S}$ molecules. The transition from lamella to perforated lamella occurs at a PS volume fraction between 0.79 and 0.82 , and the transition from perforated lamella to cylinders occurs at a PS volume fraction between 
0.82 and 0.85 . In the IY81 blends series, neither perforated lamella nor Gyroid morphology was observed. The splitting of the OOTs in the SY62 and IY81 series most likely results from the asymmetry in the ability of the homopolymer to penetrate the PI and PS brushes due to the difference in chain crowding on the two sides of the interface in the $\mathrm{I}_{2} \mathrm{~S}$ material. The homopolymer must be able to effectively penetrate this brush in order to drive the changes in interfacial curvature, which accompany an OOT. ${ }^{17,18,20}$

The SAXS data for the perforated lamellar sample (SY62-82) indicates that the layer repeat distance of the perforated lamella and the distance between adjacent perforations are both about $40 \mathrm{~nm}$. Measured from the TEM image, the PI layers are about $10 \mathrm{~nm}$ thick and the PS layers are about $30 \mathrm{~nm}$ thick. The distance between adjacent PS perforations is also about $40 \mathrm{~nm}$. From the SAXS and TEM data, we cannot distinguish between $\mathrm{ABC}$ and $\mathrm{AB}$ stacking of the perforated layers. SAXS scattering pattern of perforated lamella has been studied by several groups. . $35,37-39,46,50-52^{\text {Some }}$ analysis of these projections has been given previously by Bates and coworkers. ${ }^{38}$ However, detailed analysis of the interesting TEM projections of perforated lamella has not been reported.

Figure 2.14 illustrates the model for hexagonally perforated lamella. The upper model was proposed by Bates group. It illustrates the alternating black and white layers with the majority component (the black component in the model) perforate though the minority component (the white component) hexagonally. In this model, the perforations were drawn as solid cylinder shape. In reality, the sharp $90^{\circ}$ angles between the vertical cylinder perforations and the flat layers has high energy and cannot exist in nature. The 
perforations actually adopt catanoid shape, as shown in the lower model. Different TEM projections are possible depending upon the direction of projection within the layer of perforations and on the way in which the thin section intersects the three-dimensional structure. In general these projections reveal the layering of the structure as well as a side view of the perforations. When microtome parallel to the XY plane and viewing along $\mathrm{Z}$ axis in electron microscope, as shown in Figure 2.15, a projection of white spots in dark background is observed. The white spots are the PS perforations, and the dark background is the stained PI layer. The projection appears like a hexagonal honeycomb mesh of dark PI perforated by holes of light PS. This image is striking for its illustration of the relatively thin mesh-like structure in the PI layer that results from the low PI volume fraction of 0.18 in this blend. Since the perforations are not straight cylinder shape, the contrast between the white perforation and the dark PI layer is not sharp. The projection also cannot reflect the alternating PI PS layer structure, as the observation direction is perpendicular to $\mathrm{XY}$ plane.

Figure 2.16 illustrates the TEM images obtained when the microtoming cuts perpendicular to the layered structure and thus the TEM projection occurs parallel to the layers. The electron beam is perpendicular to the film surface and will go though the film. Since the observation direction is parallels the XY plane, the TEM projections reflect the alternating PS PI layers, which is what we observed. The PS perforations in the PI plane become white spot in the projections. However, the appearance of these perforations can appear differently depending on the microtome direction within the $\mathrm{XY}$ plane. Shown in Figure 2.16, case a, the microtomed film contains one row of PS perforations or two row of PS perforation stacking exactly over each other. They have 
the same electron transmission intensity profile, as shown in Figure 2.16, where the maximums are at the center of the PS perforations, which correspond to the white area in the schematic and TEM micrograph of Figure $2.16 \mathrm{al}$, and the distance between the adjacent maximums is the distance between PS perforations. These features are supported by the TEM micrograph a. Measured from TEM, the average distance between adjacent white spots is $40 \mathrm{~nm}$; the distance measured from SAXS is $37 \mathrm{~nm}$. Considering the accuracy of TEM measurement, this two distance agrees with each other very well. Figure 2.16, Case b show another extreme case where PS perforations of one row are positioned exactly in the middle of two PS perforations of the other row. The electron transmission intensity profile of case b shows intensity minimums at the center of the PS perforations. The height of the intensity maximums is low. And the distance between the adjacent maximums is half of the distance of the adjacent PS perforations. Reflected in the TEM projection schematics in Figure $2.16 \mathrm{~b}$, the contrast of the black spots and the gray PI layer is low; the black spots are small and near each other. These features are supported in the TEM micrograph $b$.

Figure 2.17 discusses shows TEM projections when the cutting direction is tilted. Since the grain direction of perforated lamella is usually random and constantly change inside a bulk sample. Most TEM observation reflects the microtome directions neither perpendicular to XY plane nor parallel to XY plane. Shown in Figure 2.17, as the tilting angle $\theta$ decrease from $90^{\circ}$ to $0^{\circ}$, the TEM projections change from that of Figure 2.16 to that of Figure 2.15. The dark PI layers will appear thicker and thicker as the tilting angle $\theta$ decreases. When the tilting angle is appropriate, the TEM will show regular 
projections. For example, when $\tan \theta=\mathrm{a} / \mathrm{b}$, the microtomed PI layer will contain one bright PS perforations within each dark PI layer. In the projected image, the position of the PS perforation inside the PI layer can change, as illustrated in Figure 2.17, schematic B. the PS perforation appears to be two half with spots in the both sides of a dark PI layer. Further decrease the tilting angle $\theta$, the black PI layer band will appears thicker and the white PS layer band in the projection will appears thinner in the projected image. And the differences in thickness will increase with the decreasing of tilting angle $\theta$. The projection in TEM will feature more multi-perforation in PI layers. When $d / \cos \theta=2 \mathrm{a} / \mathrm{b}$, the projection will contain 2 adjacent bright PS perforations in PI layer; when the tilting angle is even smaller $(2 \mathrm{a} / \mathrm{b}<\mathrm{d} / \cos \theta)$, the PI layer will contain more than 2 PS perforations in the projection. In reality, due to the constant changing of the grain direction, the TEM image of Figure 2.17 shows a combination of those features. As marked on the TEM micrograph, position $\mathrm{A}, \mathrm{B}, \mathrm{C}$, and $\mathrm{D}$ reflects the projection of schematic $\mathrm{A}, \mathrm{B}, \mathrm{C}$, and $\mathrm{D}$ respectively. In 3 dimension, the microtome direction can actually tilt in $\mathrm{YZ}$ other direction as well and the combination of these two tilting will lead to more complex projections. As the result, the TEM images of perforated lamella contain the regular ones mention above and more irregular projection, all of them reflect the structure features of alternating layers and the perforation of the majority component perforated though the minority hexagonally.

In previously reported vesicle forming block copolymer / homopolymer blend systems, employing a relatively high molecular weight homopolymer, the homopolymer is the major component and the block copolymer forms a bilayer in the homopolymer 
matrix. ${ }^{97}$ That structure is analogous to the classical vesicle structures in surfactant systems. Although the morphology of the IY89-82 blend (Figure 2.11) has a vesicle-like appearance, its structure is inverted from the standard vesicle structure. The block copolymer amphiphile forms the matrix, both inside and outside of the vesicles, while the minor component homopolymer resides in the double layer formed by the short PI blocks.

With the addition of increasing amounts of hPI in the IY89 series samples IY8980 to IY89-71, the PI sheets forming the closed vesicles in IY89-82 become connected in a network of randomly ordered sheets that appear to form sample spanning structures, at least on the scale of our TEM observation. We speculate that the transition from a structure of predominantly closed vesicles and isolated sheets in IY89-82 to a structure dominated by continuous sheets at higher hPI content may be a percolation phenomenon. During microphase separation of the sheet-like PI structure there will be an energetic driving force to avoid free sheet edges. This can be accomplished by either forming closed vesicles or by producing a semi-infinite network of sheets. During the microphase separation process at lower overall PI content (IY89-82) embryonic pieces of microphase separating sheet structure do not find enough neighboring sheet material to form a network and thus tend to close on themselves to produce vesicles. At slightly higher overall PI content, the percolation threshold is apparently reached and the microphase separating sheets "find" enough like structure in the system to link up into a continuous network. Above the percolation threshold, there is no obvious morphology change from IY89-80 to IY89-71. 
In blends of asymmetric $\mathrm{I}_{2} \mathrm{~S}$ block copolymer and homopolymers of either PS or PI, the order-order transitions were found to occur at different volume fractions depending on which type of homopolymer was used in the blends. Measurements of domain spacings showed that the PI homopolymer does not penetrate as well into the two-arm per molecule PI brush of the $\mathrm{I}_{2} \mathrm{~S}$ as does PS homopolymer into the one-arm per molecule PS brush on the PS side of the interface. This asymmetry of homopolymer brush penetration brought about by the asymmetric architecture of the graft copolymer leads to the splitting of the OOTs. Some of the blends produced interesting and unusual morphologies. For instance sample SY62-82 formed well ordered perforated lamella in which the perforated layers comprised only 18 volume percent of the structure. This perforated lamellar morphology was found to be stable to thermal annealing, which indicated that perforated lamella could be stable at certain composition. Unusual vesicle and continuous folded sheet morphologies were found in other blends. A transition been closed vesicles and continuous sheet structures was observed as a function of the overall concentration of the PI, which forms the vesicle or sheet walls. This may result from percolation effects. When the PI concentration is lower, there is not enough material to form a continuous network of sheets, and isolated vesicles form instead. At higher PI concentrations a percolation threshold is reached and the sheet structures can link up into a network. 
Table 2.1. Molecular characterization of the $\mathrm{I}_{2} \mathrm{~S}$ block copolymer and the PS and PI homopolymers.

a membrane osmometry; b SEC; c Vol\% calculated utilizing density of (d-PS) $=1.14$ $\mathrm{g} / \mathrm{ml}$ at $250 \mathrm{C}$ and density of PI $=0.91 \mathrm{~g} / \mathrm{ml}$ at $25 \mathrm{oC}^{98}$ along with the weight percent compositions from SEC-UV.

\begin{tabular}{|c|c|c|c|c|c|c|}
\hline Sample Name & $\begin{array}{c}{ }^{\mathrm{a}} \mathrm{M}_{\mathrm{n}}(\mathrm{PS} \text { arm }) \\
\left(\times 10^{-3}\right)\end{array}$ & $\begin{array}{c}{ }^{\mathrm{a}} \mathrm{M}_{\mathrm{n}}(\mathrm{PI} \text { arm }) \\
\left(\times 10^{-3}\right)\end{array}$ & $\begin{array}{c}{ }^{\mathrm{a}} \mathrm{M}_{\mathrm{n}} \\
(\text { total })\end{array}$ & ${ }^{\mathrm{b}} \mathrm{M}_{\mathrm{w}} / \mathrm{M}_{\mathrm{n}}$ & $\begin{array}{c}{ }^{\mathrm{c}} \mathrm{Vol}_{\%} \\
\mathrm{~d}-\mathrm{PS}\end{array}$ & $\begin{array}{c}{ }^{\mathrm{d}} 2 \pi / \mathrm{q}^{*} \\
( \pm \\
0.5 \mathrm{~nm})\end{array}$ \\
\hline $\mathrm{I}_{2} \mathrm{~S}-89$ & 87.3 & $4.6^{\mathrm{e}}$ & $97.1 \times 10^{3}$ & 1.04 & 89 & \\
\hline $\mathrm{I}_{2} \mathrm{~S}-81$ & 79.1 & 9.6 & $89.4 \times 10^{3}$ & 1.04 & 81 & 31.0 \\
\hline $\mathrm{I}_{2} \mathrm{~S}-62$ & 61.2 & 14.8 & $83.0 \times 10^{3}$ & 1.04 & 62 & 39.0 \\
\hline $\mathrm{hPS}$ & & & 870 & 1.03 & & \\
\hline $\mathrm{hPI}$ & & & 872 & 1.17 & & \\
\hline
\end{tabular}


Table 2.2. Composition and Morphology of the blends in the study.

${ }^{a}$ Calculated utilizing density of $(\mathrm{d}-\mathrm{PS})=1.14 \mathrm{~g} / \mathrm{ml}$ at $25^{\circ} \mathrm{C}$ and density of PI $=0.91$ $\mathrm{g} / \mathrm{ml}$ at $25^{\circ} \mathrm{C}^{98} \mathrm{~b}^{*} \mathrm{q}^{*}=4 \pi / \lambda\left(\sin \theta_{1}\right)$ and $2 \theta_{1}$ is the scattering angle for the lowest angle Bragg peak; corresponds to $d_{(100)}$ for hexagonal packed cylinders, $d_{(001)}$ for lamellae, respectively.

\begin{tabular}{|c|c|c|c|c|c|}
\hline $\begin{array}{c}\text { Sample } \\
\text { Name }\end{array}$ & $\begin{array}{c}\text { Weight } \\
\text { Fraction of } \\
\text { Homopolymer }\end{array}$ & $\begin{array}{c}\text { Overall Weight } \\
\text { Fraction of PS }\end{array}$ & $\begin{array}{c}{ }^{\mathrm{a}} \text { Overall } \\
\text { Volume } \\
\text { Fraction of PS }\end{array}$ & $\begin{array}{c}\text { Morphology } \\
( \pm 0.5 \mathrm{~nm})\end{array}$ \\
\hline IY89-86 & 0.032 & 0.88 & 0.86 & Worm & \\
\hline IY89-82 & 0.068 & 0.85 & 0.82 & Vesicle and Sheet & \\
\hline IY89-80 & 0.081 & 0.83 & 0.80 & $\begin{array}{c}\text { Disordered layer and } \\
\text { Vesicle }\end{array}$ & \\
\hline IY89-77 & 0.12 & 0.80 & 0.77 & $\begin{array}{c}\text { Disordered layer and } \\
\text { Vesicle }\end{array}$ & \\
\hline IY89-71 & 0.17 & 0.75 & 0.71 & Disordered layer and & Vesicle \\
\hline IY81-80 & 0.013 & 0.83 & 0.80 & Cylinder & 33.3 \\
\hline IY81-76 & 0.045 & 0.80 & 0.76 & Cylinder & 33.3 \\
\hline IY81-73 & 0.083 & 0.77 & 0.73 & Cylinder & 34.5 \\
\hline IY81-71 & 0.12 & 0.75 & 0.71 & Cylinder & 35.2 \\
\hline IY81-66 & 0.16 & 0.71 & 0.66 & Cylinder & 37.5 \\
\hline IY81-65 & 0.17 & 0.70 & 0.65 & Lamella & 40.4 \\
\hline SY81-82 & 0.062 & 0.85 & 0.82 & Cylinder & 31.6 \\
\hline SY81-85 & 0.25 & 0.88 & 0.85 & Disordered Cylinder & 30.6 \\
\hline SY62-73 & 0.30 & 0.77 & 0.73 & lamella & 38.5 \\
\hline SY62-76 & 0.39 & 0.80 & 0.76 & lamella & 39.4 \\
\hline SY62-79 & 0.48 & 0.83 & 0.79 & lamella & 40.0 \\
\hline SY62-82 & 0.54 & 0.85 & 0.82 & Perforated Lamella & 37.5 \\
\hline SY62-85 & 0.64 & 0.88 & 0.85 & Cylinder & 35.2 \\
\hline SY62-89 & 0.72 & 0.91 & 0.89 & Cylinder & 36.2 \\
\hline
\end{tabular}


Table 2.3. Table of brush heights and swelling ratios of the blend samples.

\begin{tabular}{|c|c|c|c|c|c|c|}
\hline & $H_{\text {PI }}(\AA)$ & $H_{P S}(\AA)$ & $\lambda^{\prime \prime}$ & $\lambda^{\perp}$ swollen phase & $\lambda^{\perp}$ unswollen phase & $K$ \\
\hline $\mathrm{I}_{2} \mathrm{~S}-81$ & 81.9 & 106.0 & 1.00 & 1.00 & 1.00 & 1.00 \\
\hline $\mathrm{IY} 81-80$ & 90.3 & 111.6 & 0.99 & 1.10 & 1.06 & 1.11 \\
\hline $\mathrm{IY} 81-76$ & 98.9 & 103.0 & 1.04 & 1.21 & 1.00 & 1.17 \\
\hline $\mathrm{IY} 81-73$ & 108.7 & 100.5 & 1.09 & 1.33 & 0.99 & 1.22 \\
\hline IY81-71 & 114.9 & 98.5 & 1.11 & 1.40 & 0.99 & 1.26 \\
\hline IY81-66 & 132.6 & 94.8 & 1.16 & 1.62 & 0.98 & 1.39 \\
\hline \multicolumn{7}{|l|}{} \\
\hline $\mathrm{I}_{2} \mathrm{~S}-81$ & 81.9 & 106.0 & 1.00 & 1 & 1.00 & 1.00 \\
\hline SY81-82 & 81.3 & 153.4 & 1.00 & 1.03 & 0.99 & 1.03 \\
\hline SY81-85 & 71.9 & 155.4 & 1.07 & 1.05 & 0.88 & 0.98 \\
\hline \multicolumn{7}{|l|}{} \\
\hline $\mathrm{I}_{2} \mathrm{~S}-62$ & 74.1 & 120.9 & 1.00 & 1.00 & 1.00 & 1.00 \\
\hline SY62-73 & 52.0 & 140.6 & 1.19 & 1.16 & 0.70 & 0.97 \\
\hline SY62-76 & 47.2 & 149.6 & 1.25 & 1.24 & 0.64 & 0.99 \\
\hline SY62-79 & 42.1 & 158.2 & 1.33 & 1.31 & 0.57 & 0.99 \\
\hline
\end{tabular}




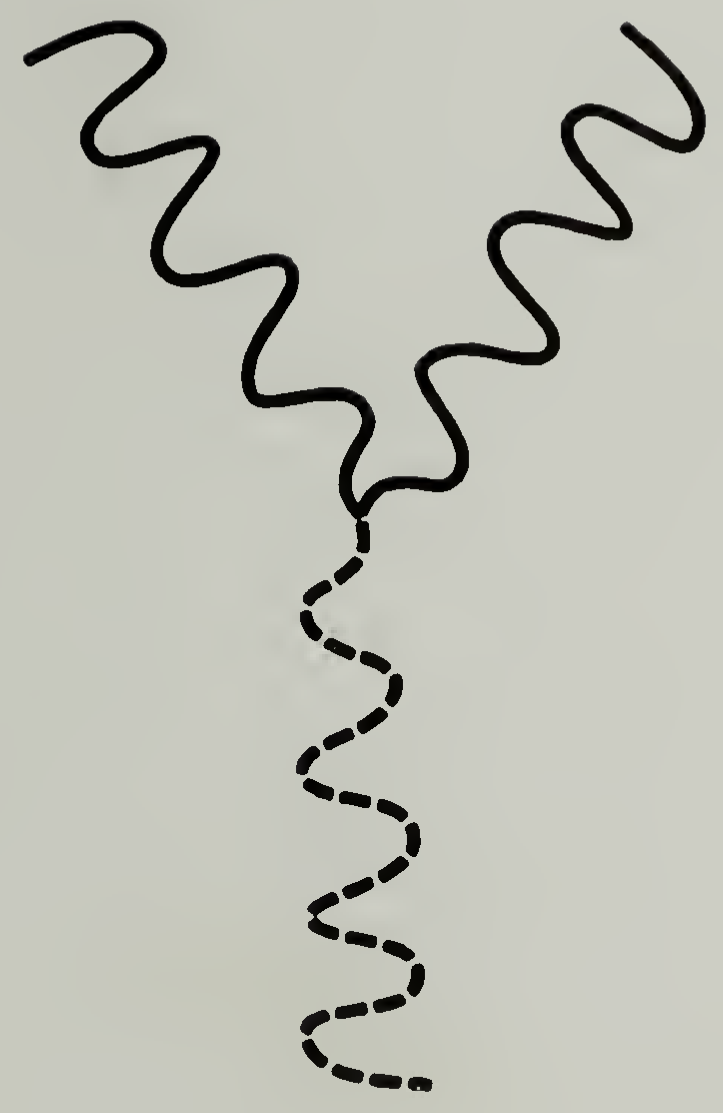

Figure 2.1. Schematic of an $\mathrm{I}_{2} \mathrm{~S}$ single graft block architecture with one PS arms (dashed) and two PI arms (solid). 


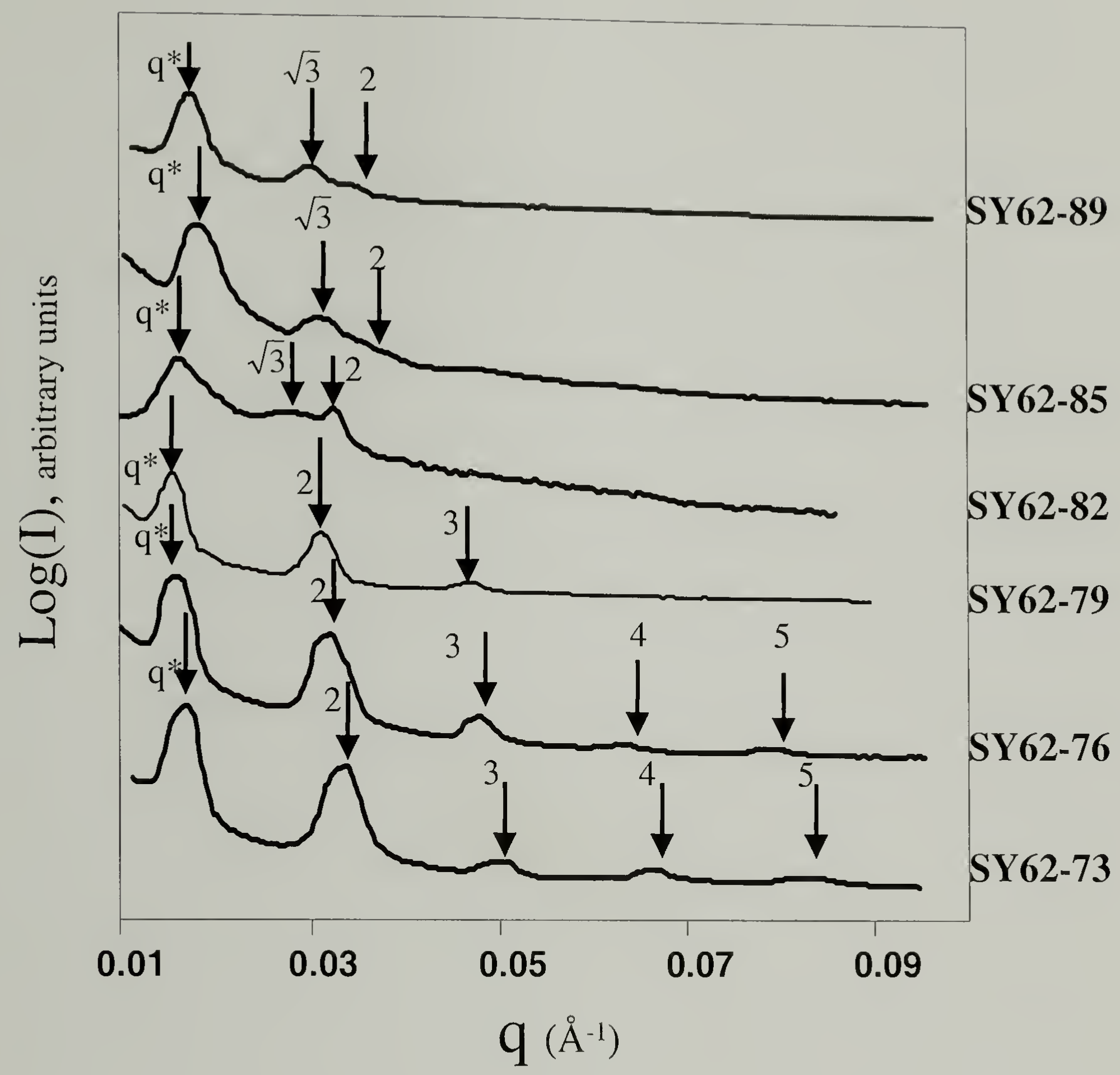

Figure 2.2. Isotropic SAXS patterns of SY62 series blends azimuthally integrated into one-dimensional plots of $\log (\mathrm{I})$ verses $\mathrm{q}$ 


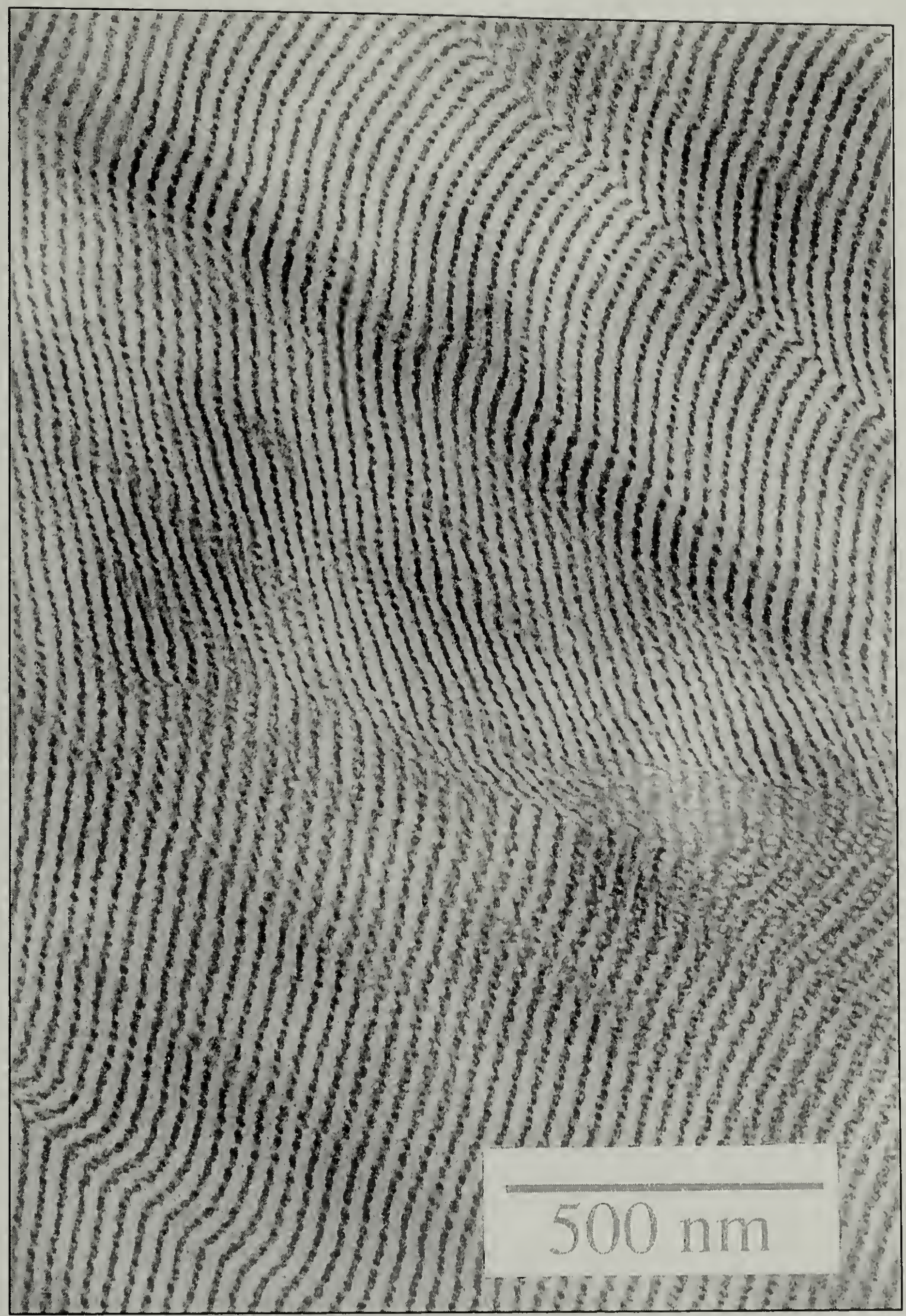

Figure 2.3. TEM images of blend SY62-82. 


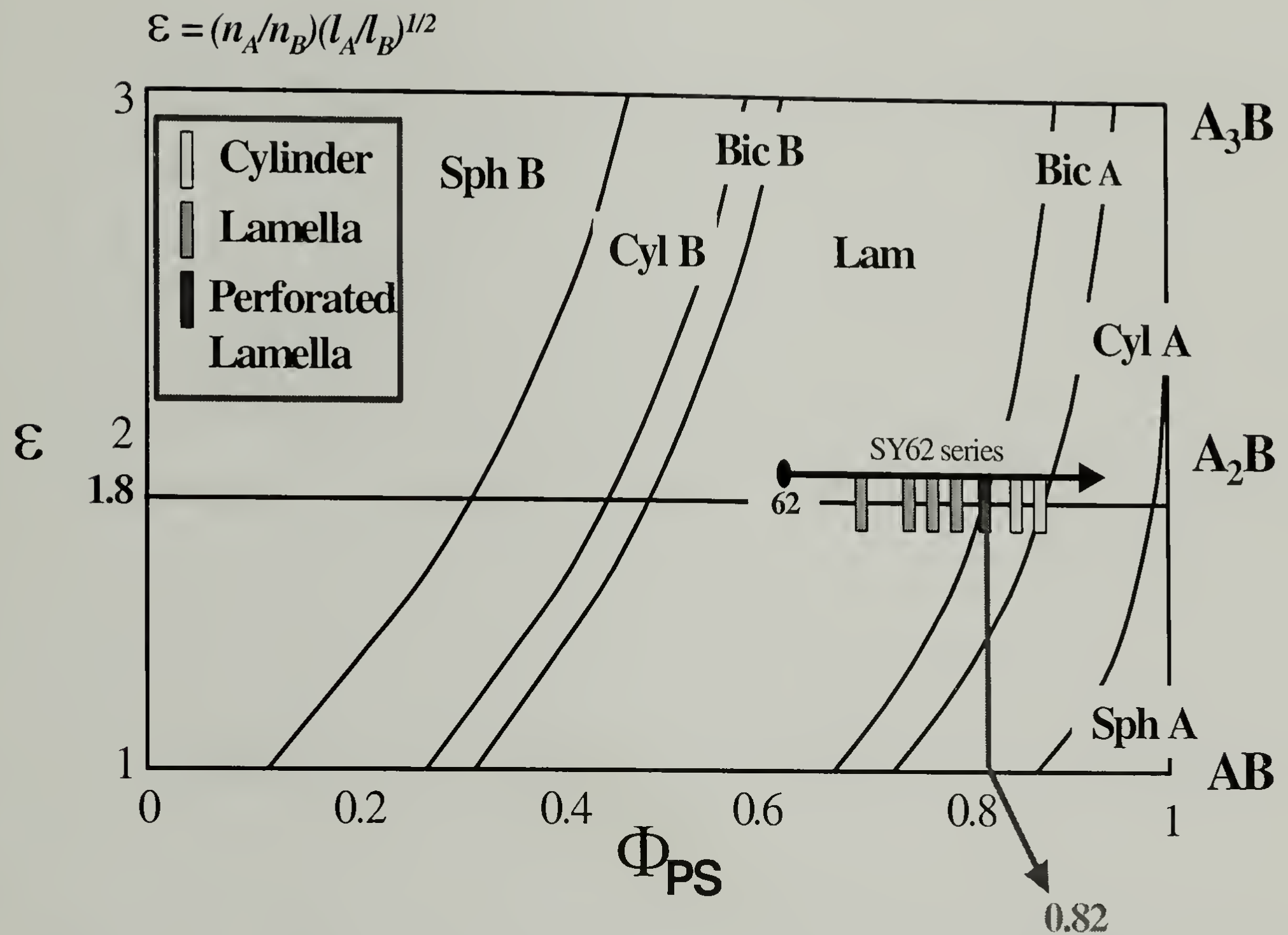

Figure 2.4. SY62 blend series mapped onto the theoretical morphology diagram. The " 62 " symbol along the solid at $\varepsilon=1.8$ indicates the volume fraction of the pure $\mathrm{I}_{2} \mathrm{~S}-62$ sample upon which the blends are based. The small rectangular boxes indicate the volume fraction of each blend in the series. The shading of the boxes indicates the morphology. 


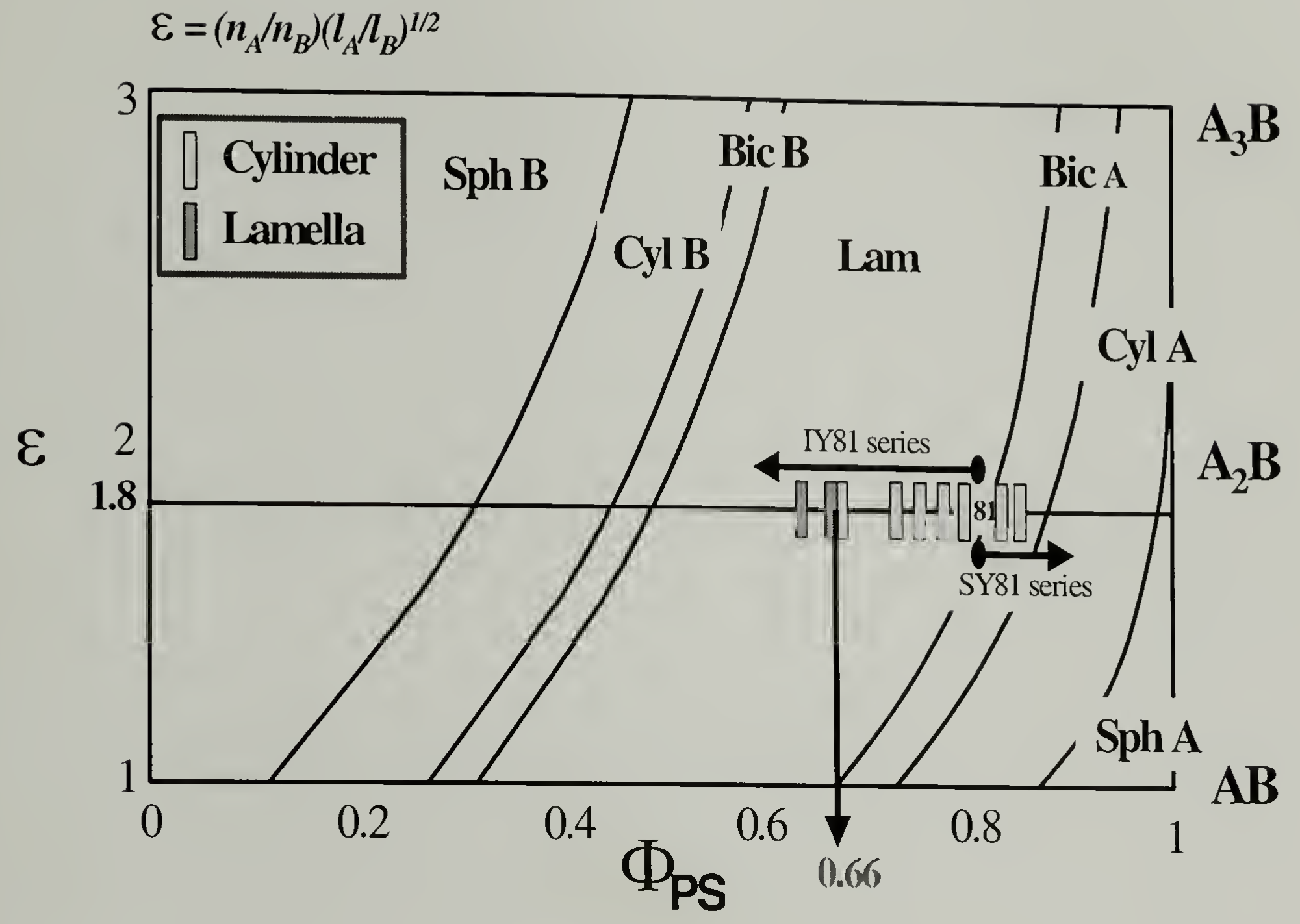

Figure 2.5. $\quad$ S81 and IY81 blend series mapped onto the theoretical morphology diagram. The " 81 " symbol along the solid at $\varepsilon=1.8$ indicates the volume fraction of the pure $\mathrm{I}_{2} \mathrm{~S}-81$ sample upon which the blends are based. The small rectangular boxes indicate the volume fractions of each blend. The shading of the boxes indicates the morphology. 


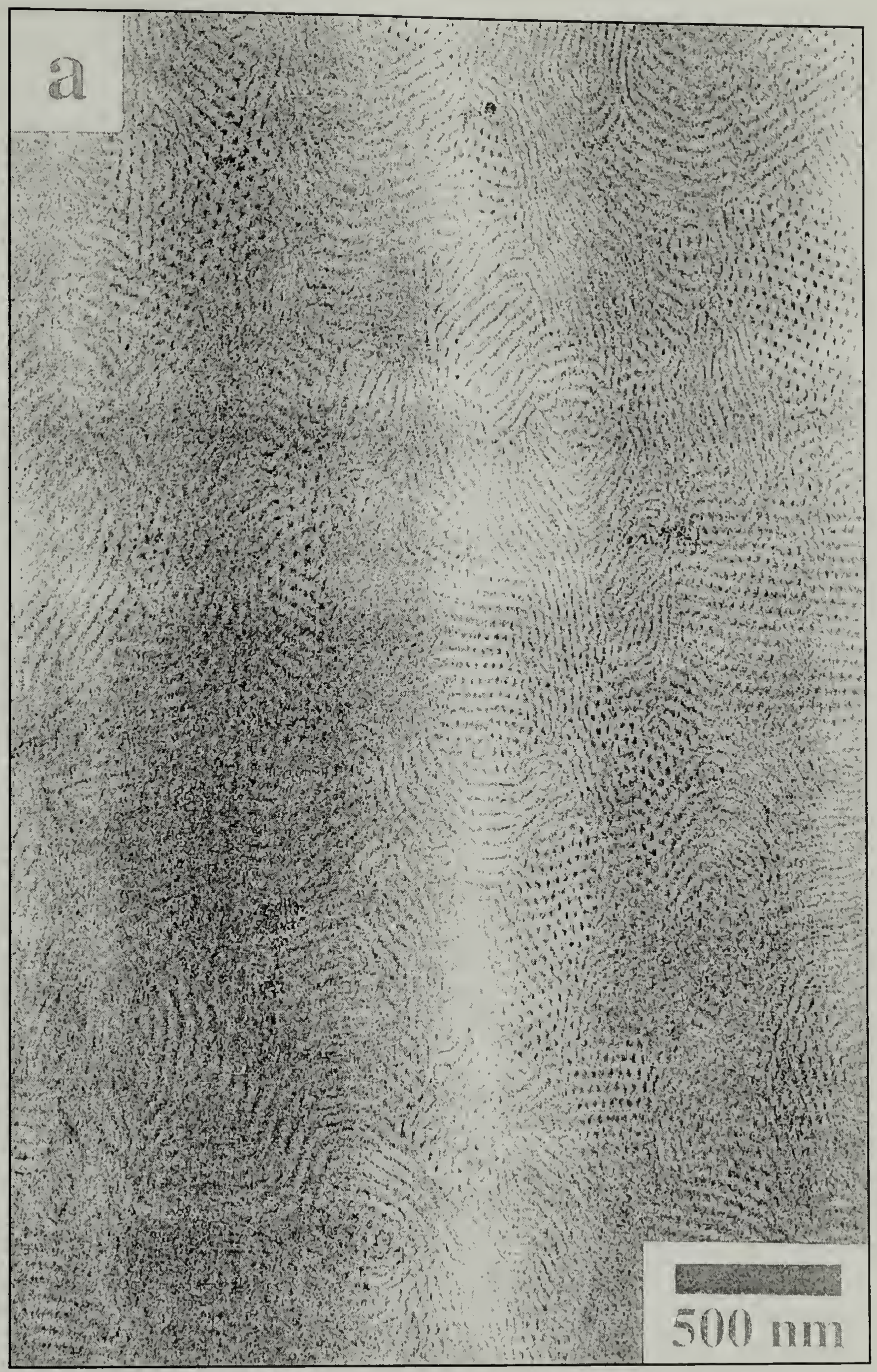




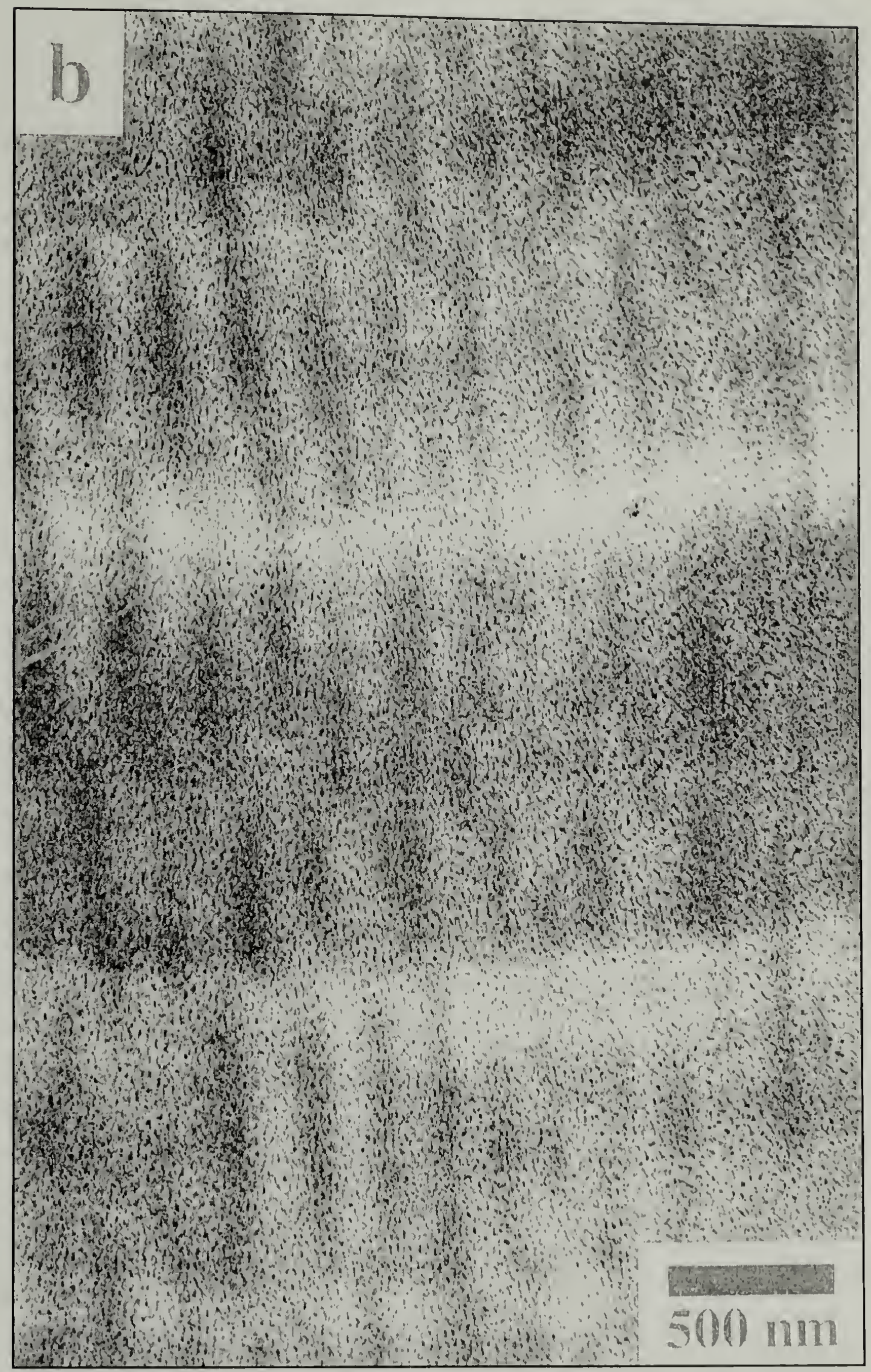

Figure 2.6. TEM micrographs of blends in the SY81 series. (a) SY81-82 showing projections both parallel and perpendicular to the PI cylinders. (b) SY81-85 showing microphase separated but not well ordered morphology. 


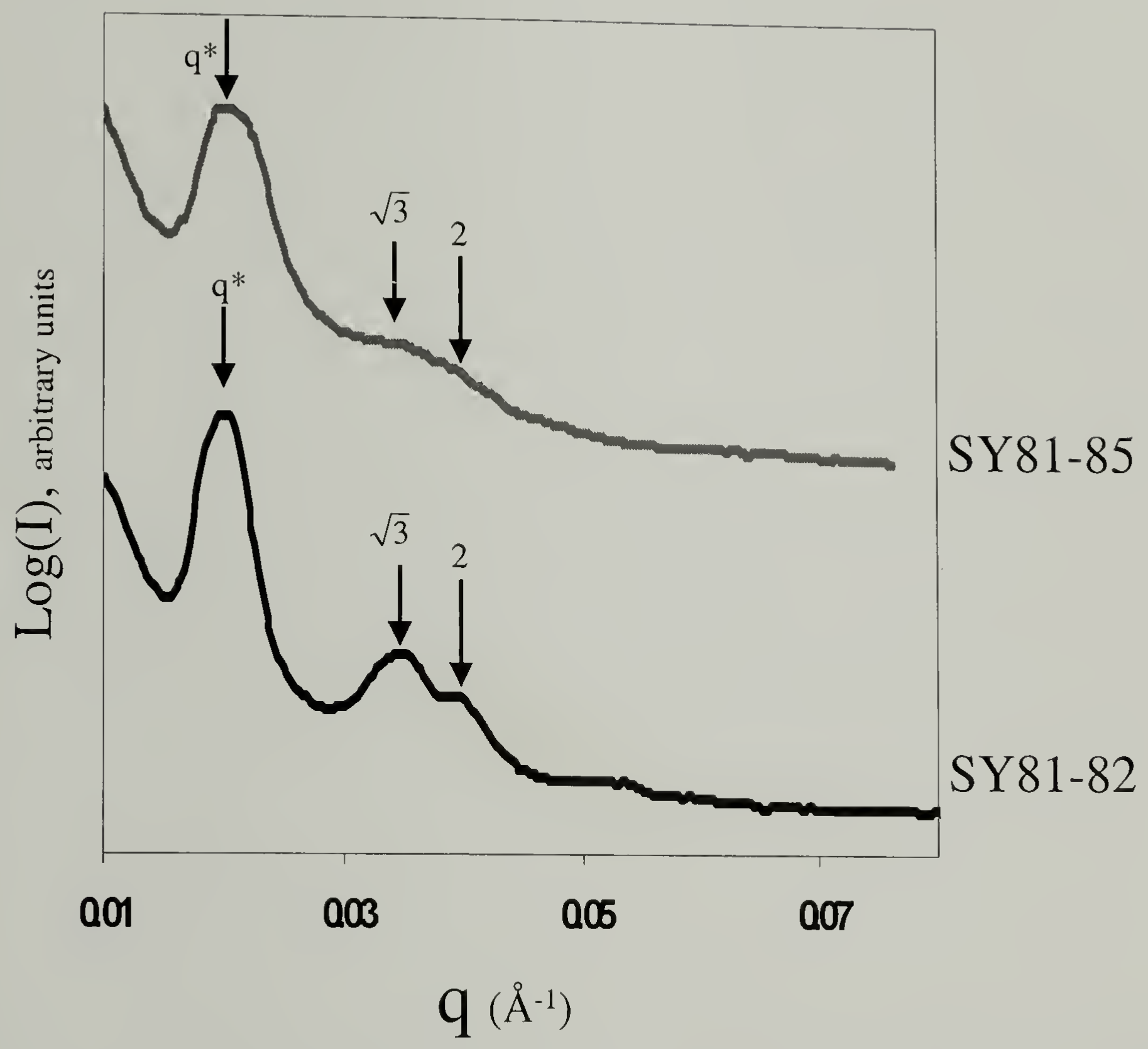

Figure 2.7. Isotropic SAXS patterns of SY81 series samples azimuthally integrated to give plots of $\log (\mathrm{I})$ verses $\mathrm{q}$. 


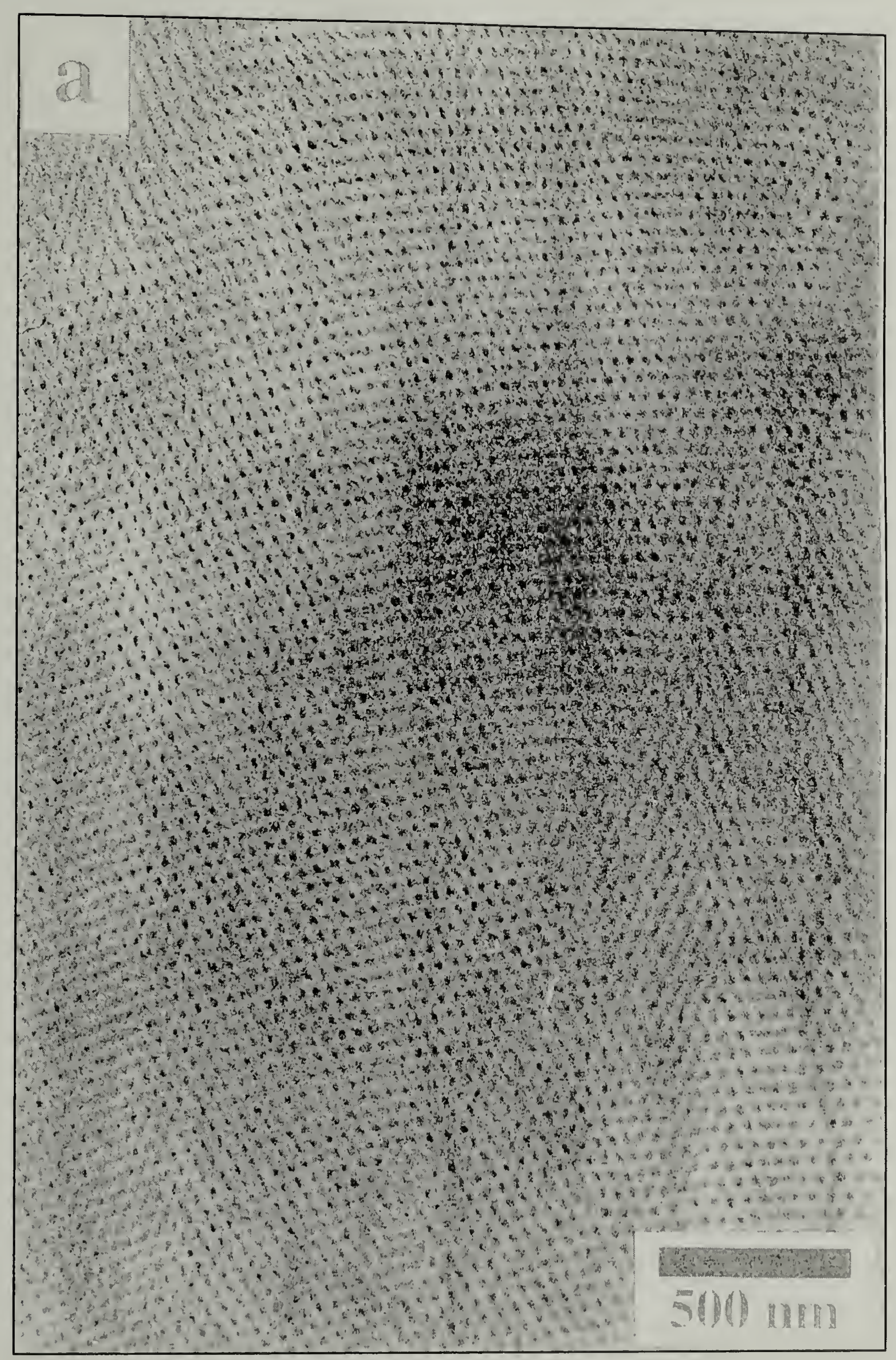




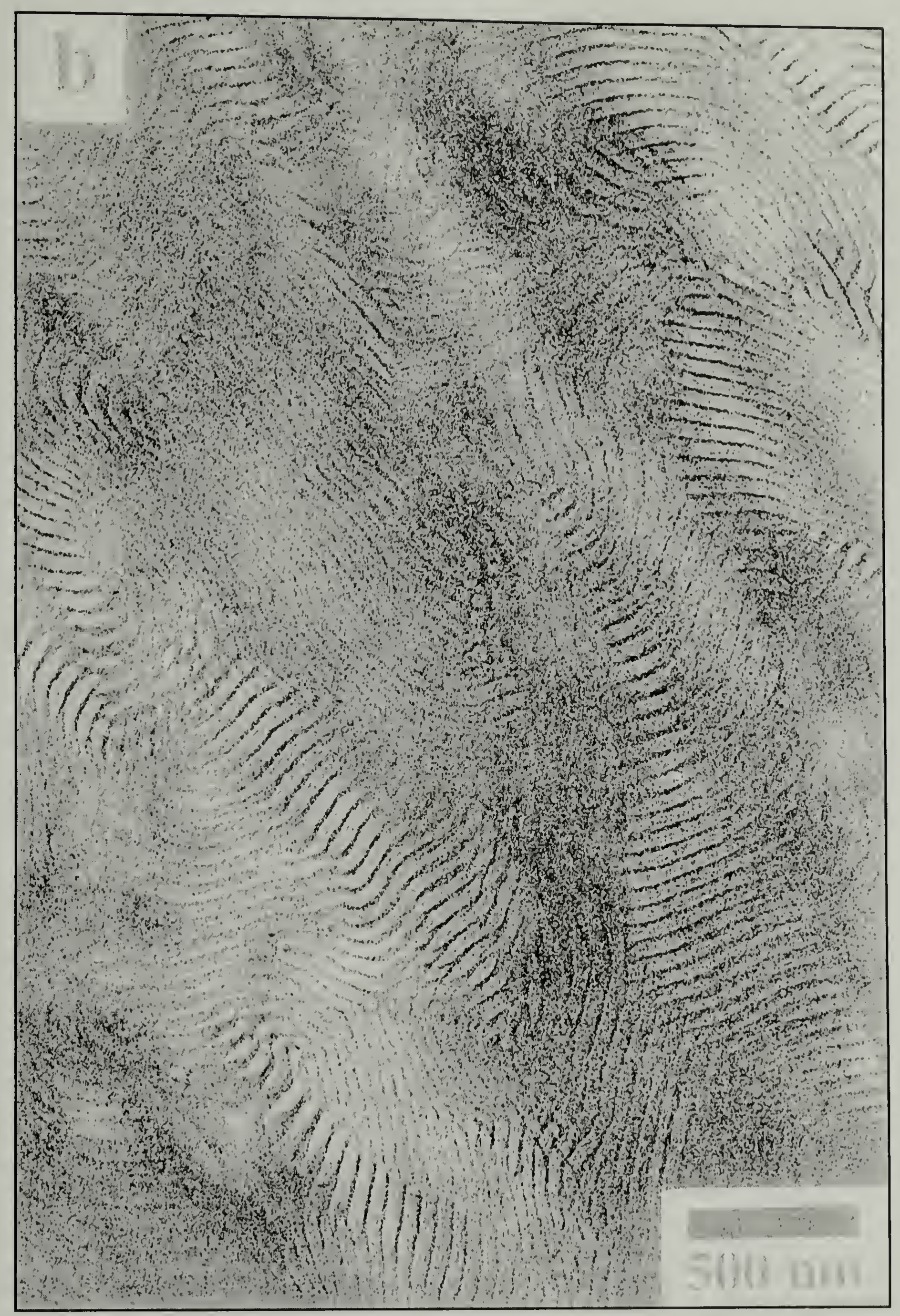

Figure 2.8. TEM micrographs from the IY81 series. (a) IY81-66 showing a projection perpendicular to PI cylinders. (b) IY81-65 showing a lamellar structure. 


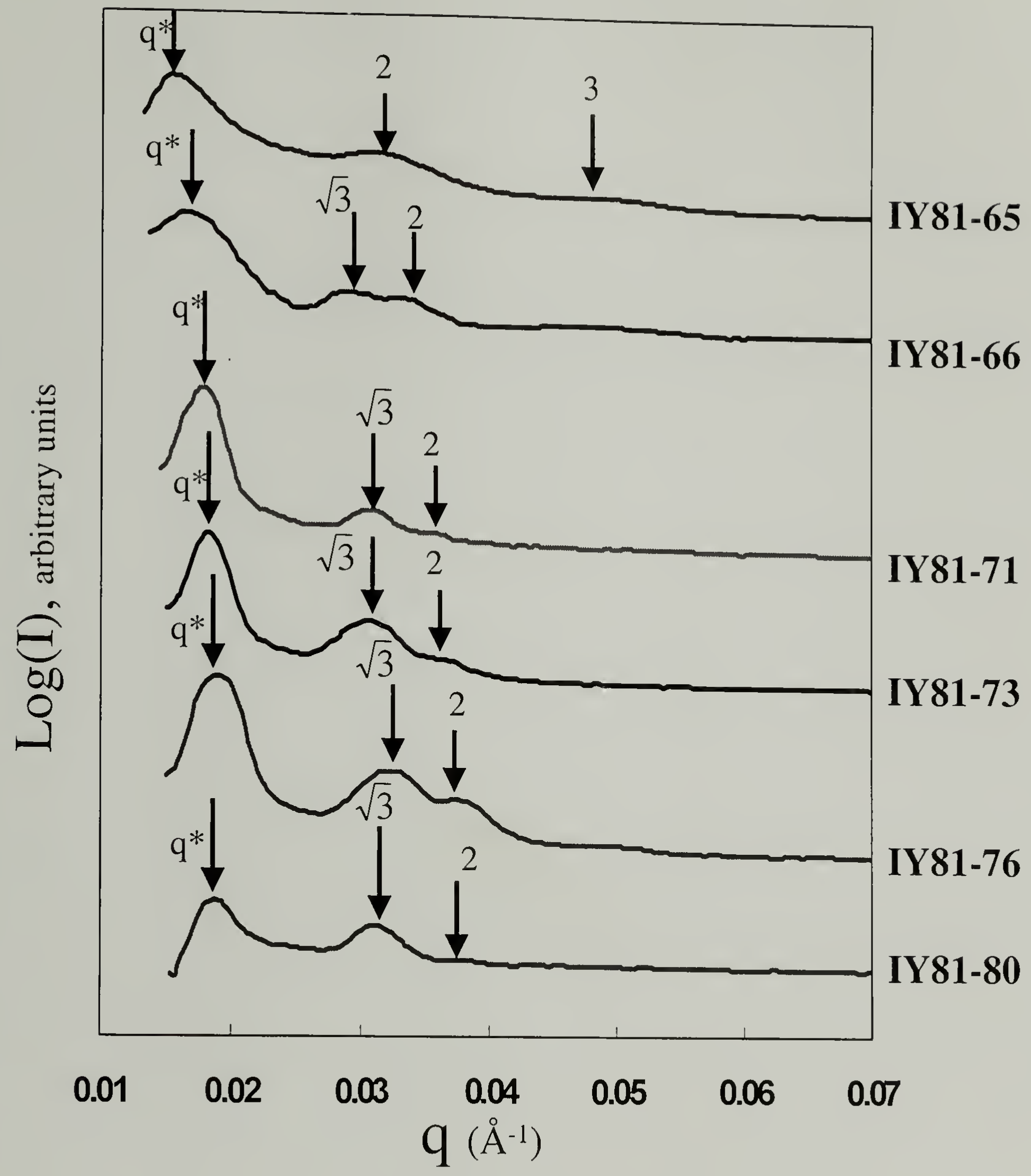

Figure 2.9. Isotropic SAXS patterns of IY81 series samples azimuthally integrated to give plots of $\log (\mathrm{I})$ verses $\mathrm{q}$. 

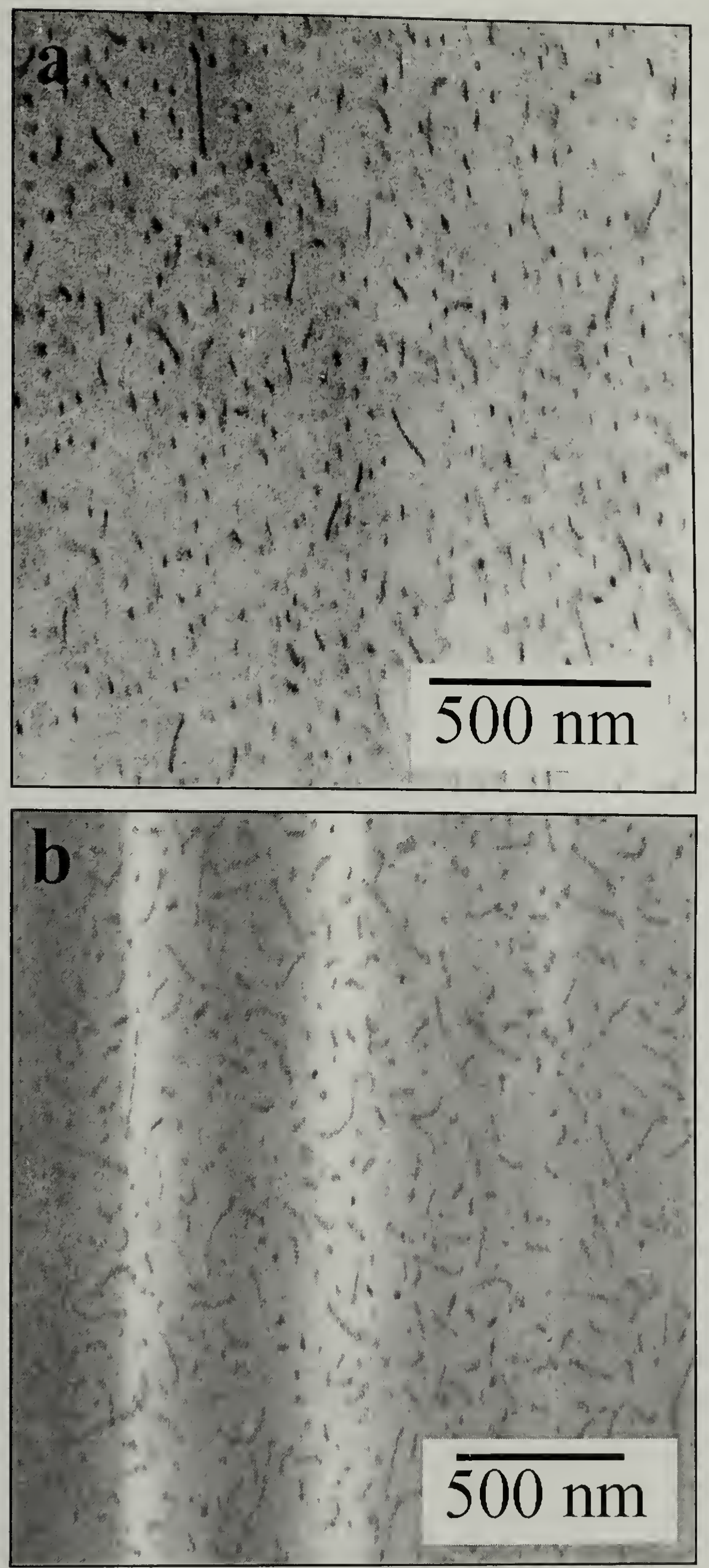

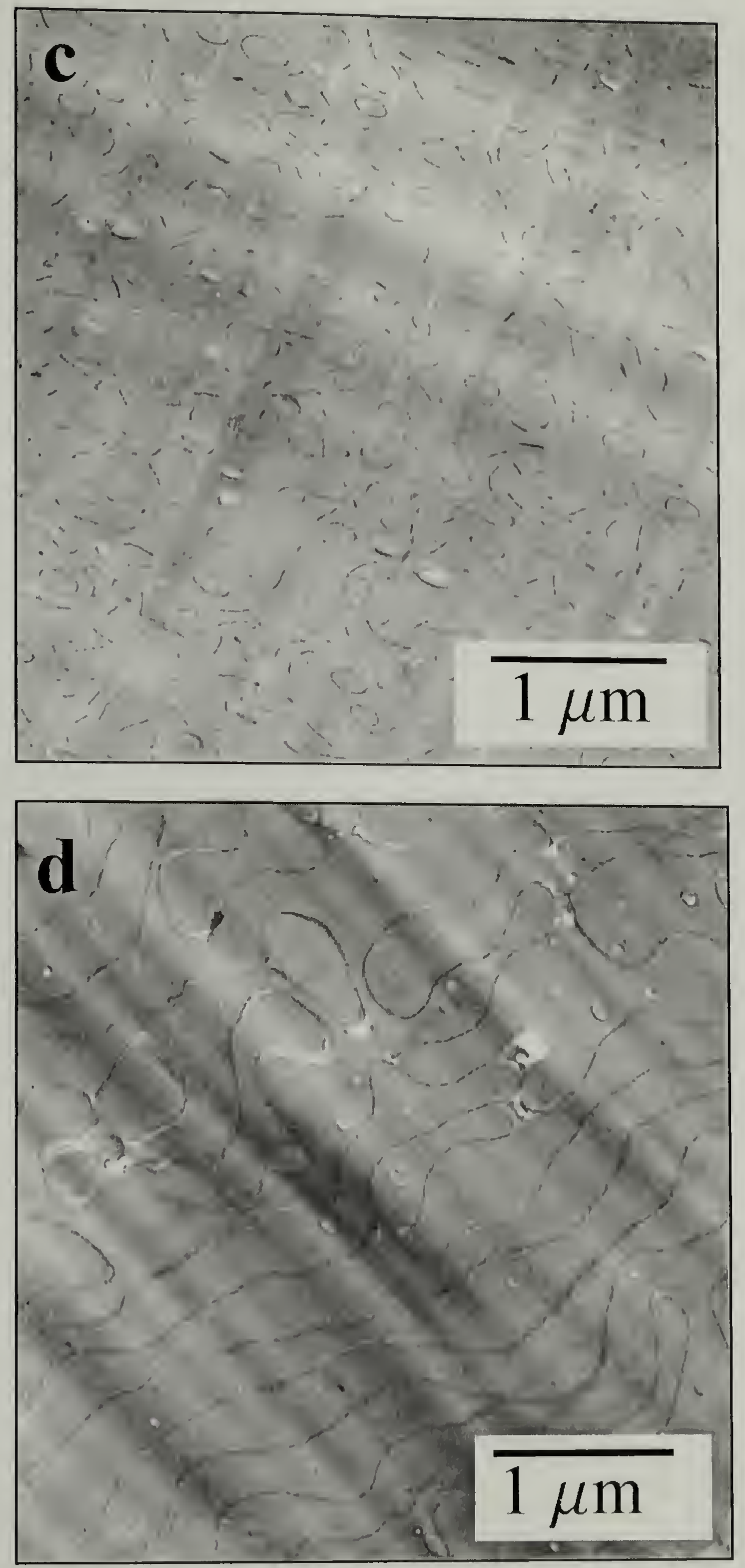


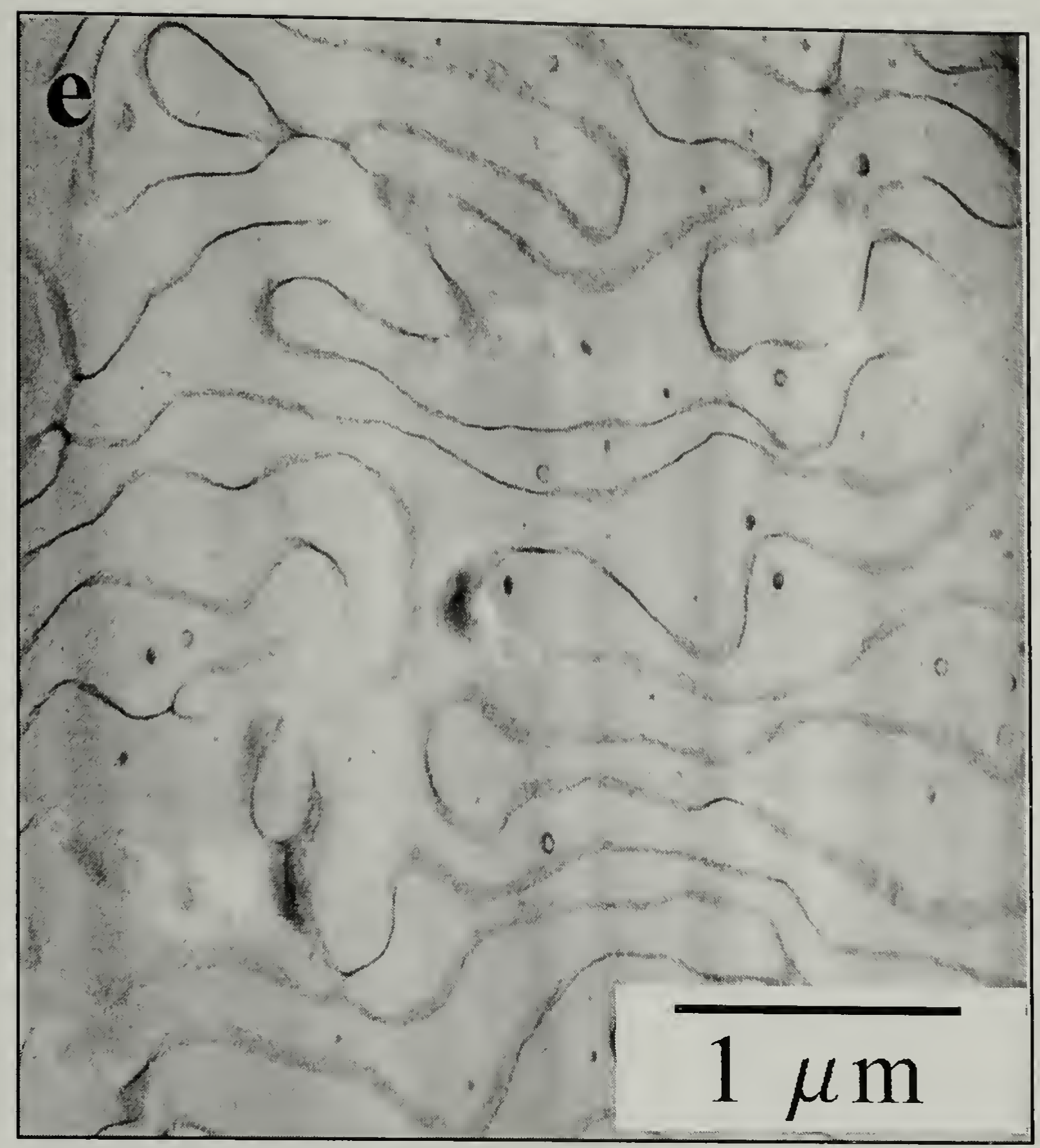

Figure 2.10. TEM image of IY89 series. a) $\mathrm{I}_{2} \mathrm{~S}-89$, b) IY89-86, C) IY89-82, d) IY8980, e) IY $89-71$ 

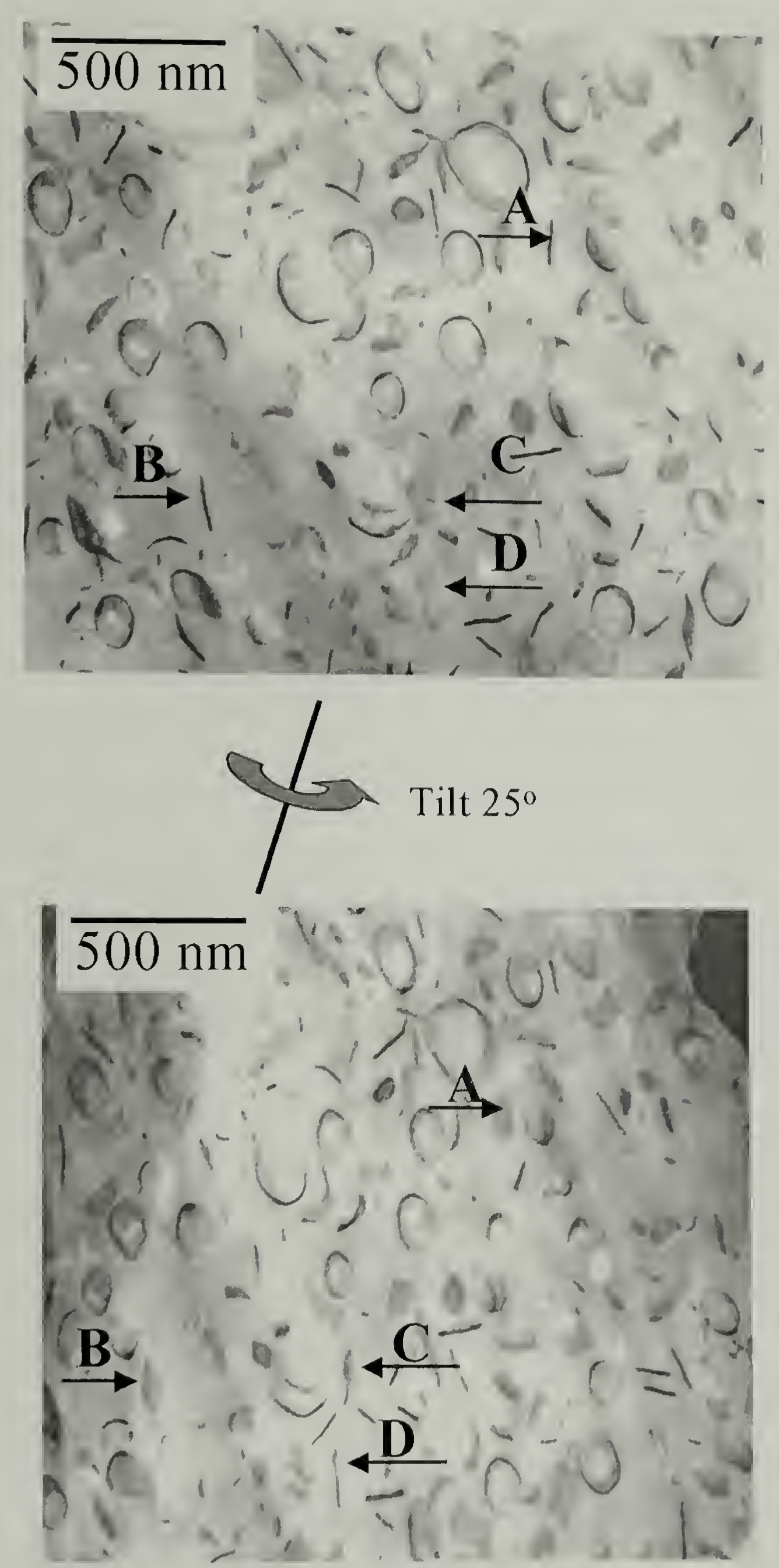

Figure 2.11. TEM tilt series of IY89-82. (a) $0^{\circ}$ tilt. (b) $25^{\circ}$ about axis indicated. After tilting, the black PI line segments A and B in (a) become PI sheets in (b). The PI sheets $C$ and $D$ in (a) become line segments in (b). 


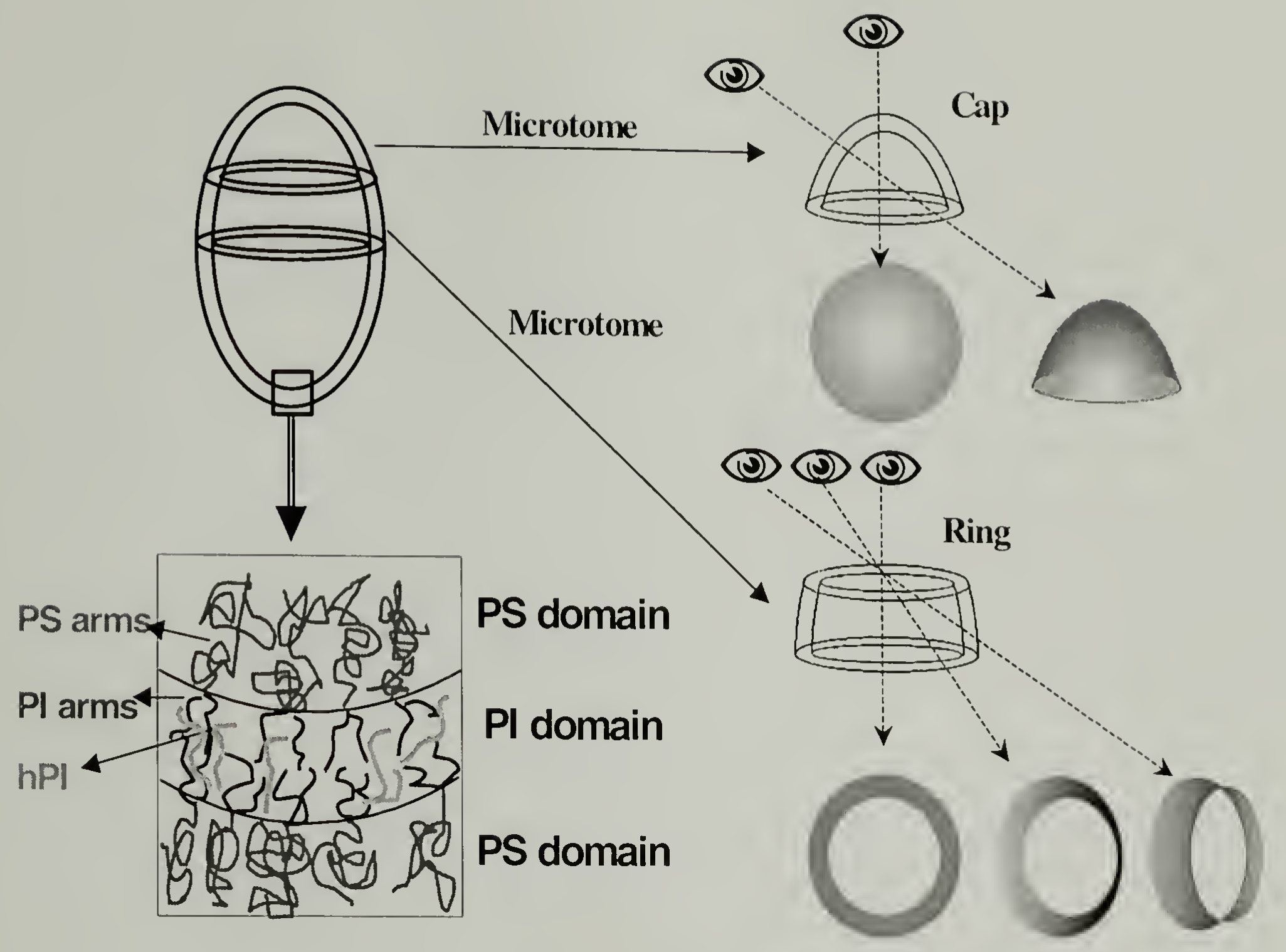

Figure 2.12. Vesicle model. After microtoming, the tops and bottoms of vesicles will appear like caps in TEM projection; the middle parts of vesicles will appear like rings. The rings and caps have different projections with the change of viewing angles and microtome positions. 


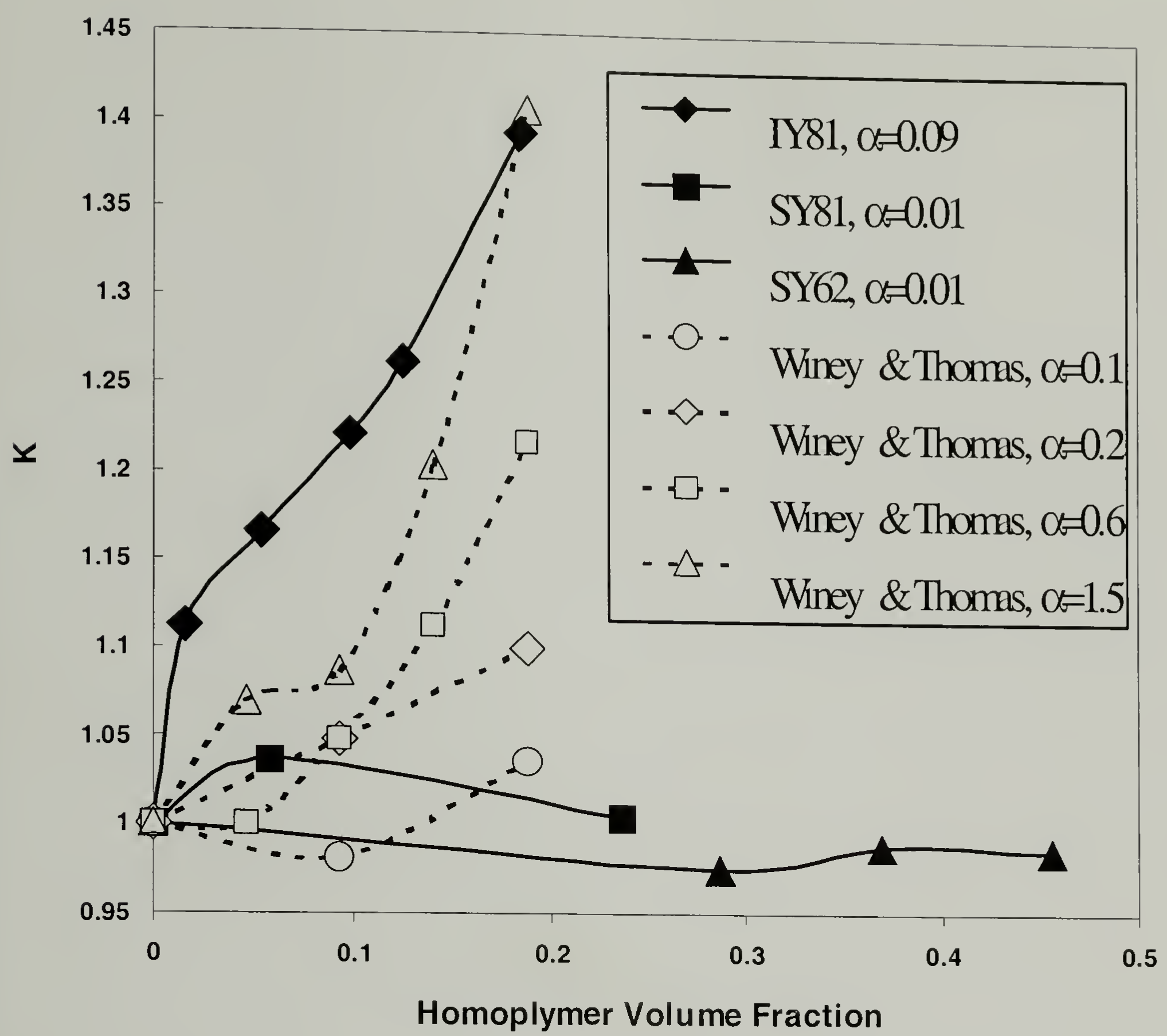

Figure 2.13. Plot of asymmetry swelling ratio, K, verses homopolymer volume fraction for the miktoarm block copolymer / homopolymer blends of this study (filled symbols) and the linear diblock / homopolymer blends studies by Winey, Thomas, and Fetters $^{19}$ (open symbols). 


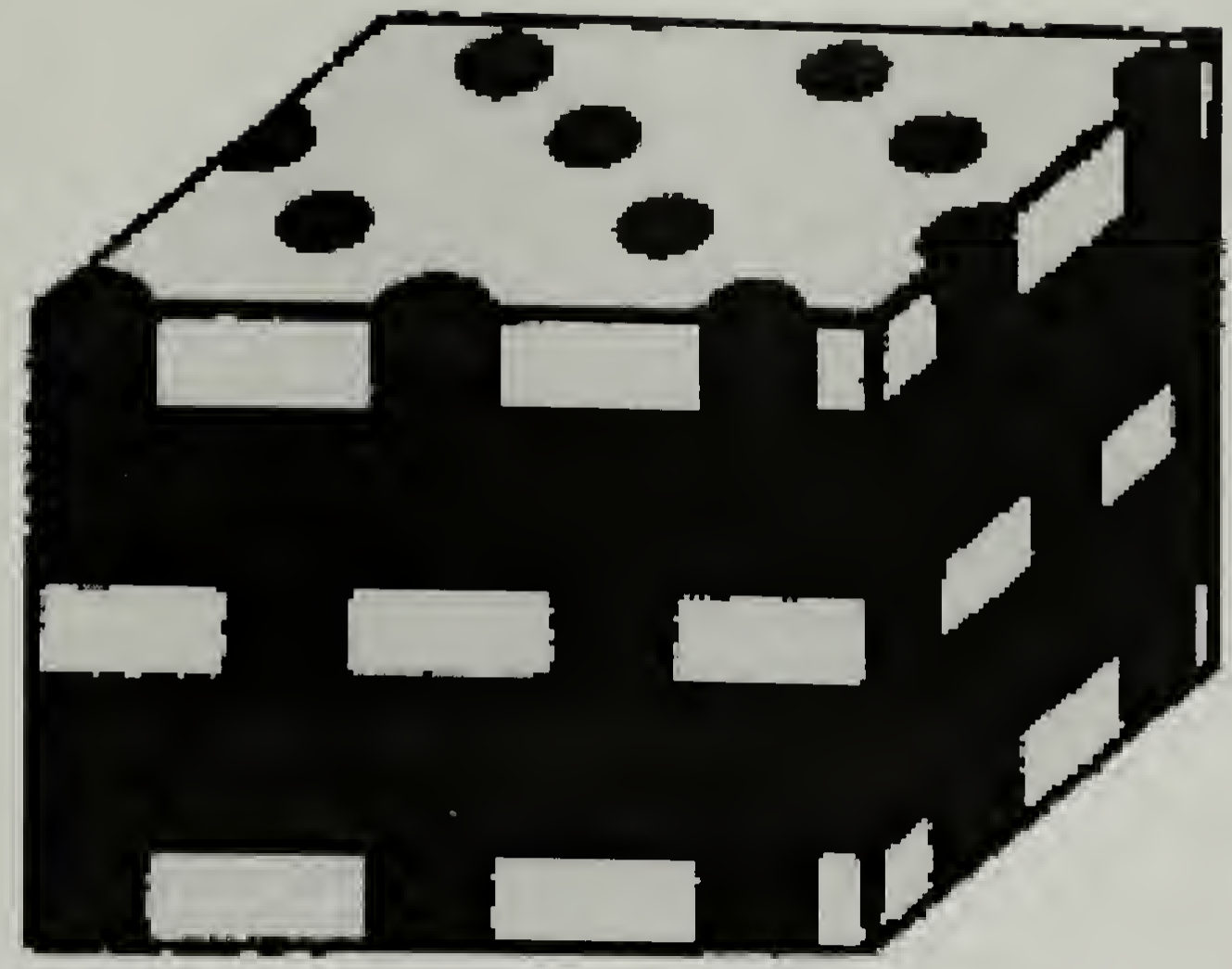

A model suggested by Forster et al . (Macromolecule, 1994. 27 6922)

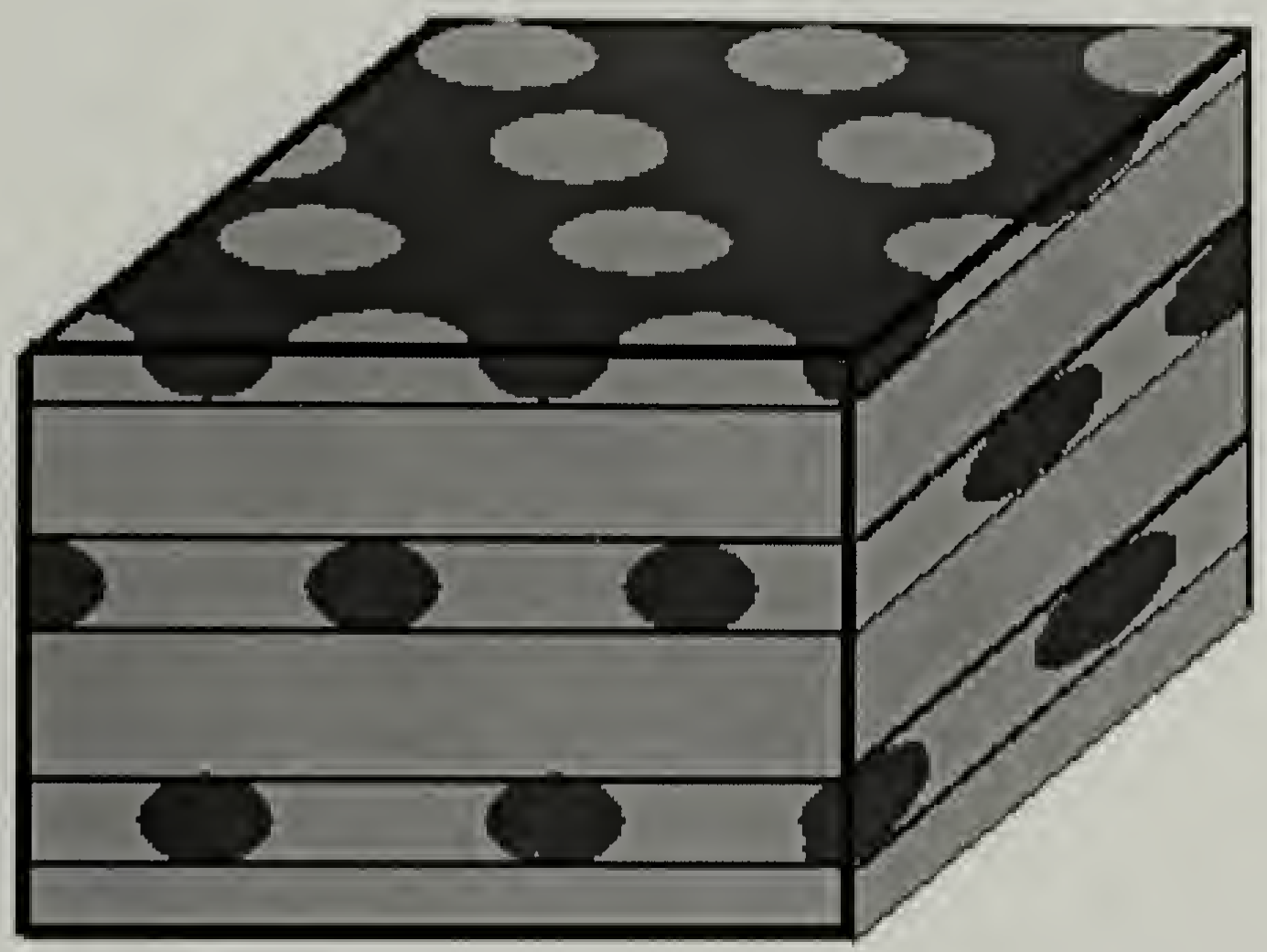

Figure 2.14. Hexagonally perforated lamella model. 

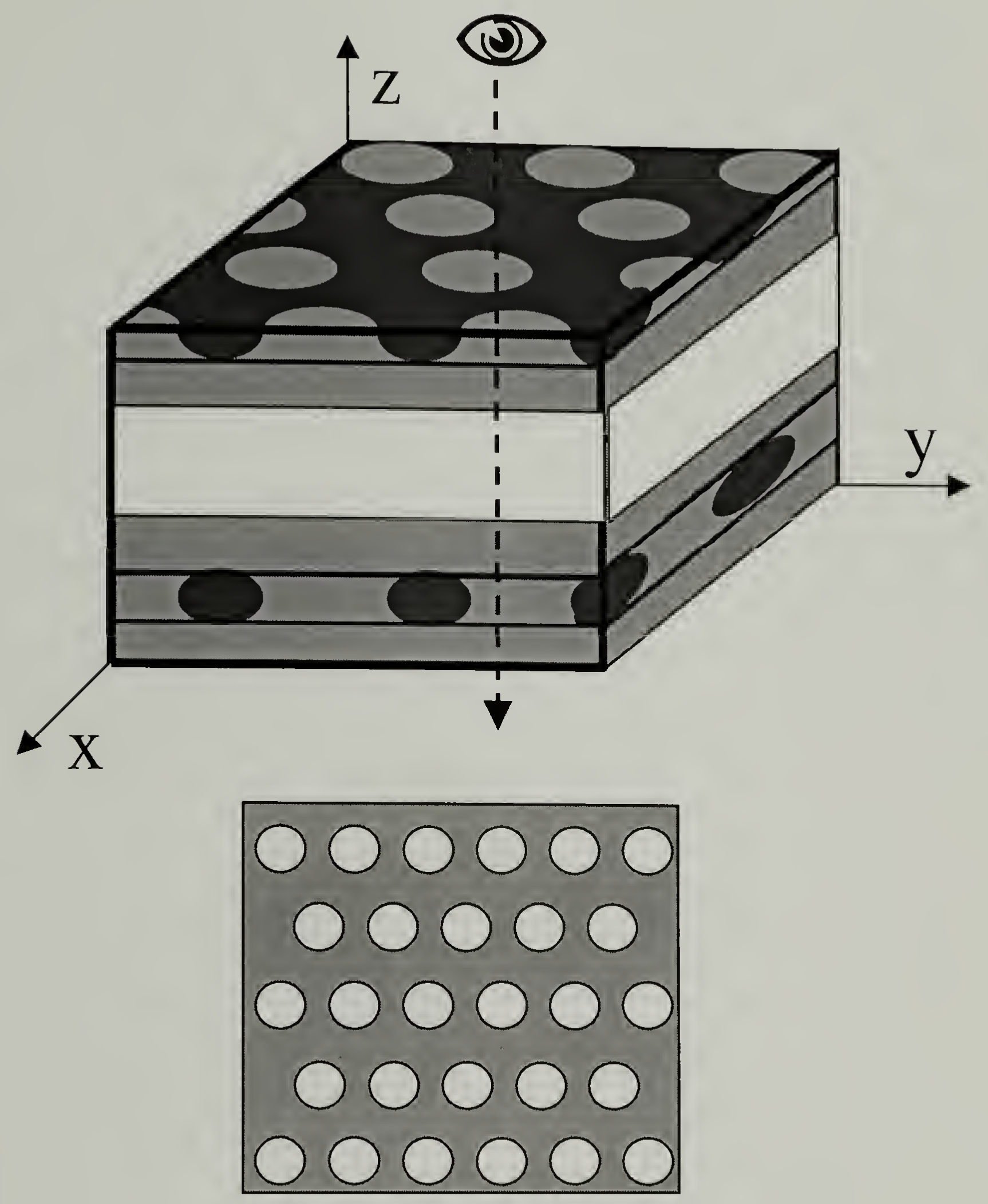


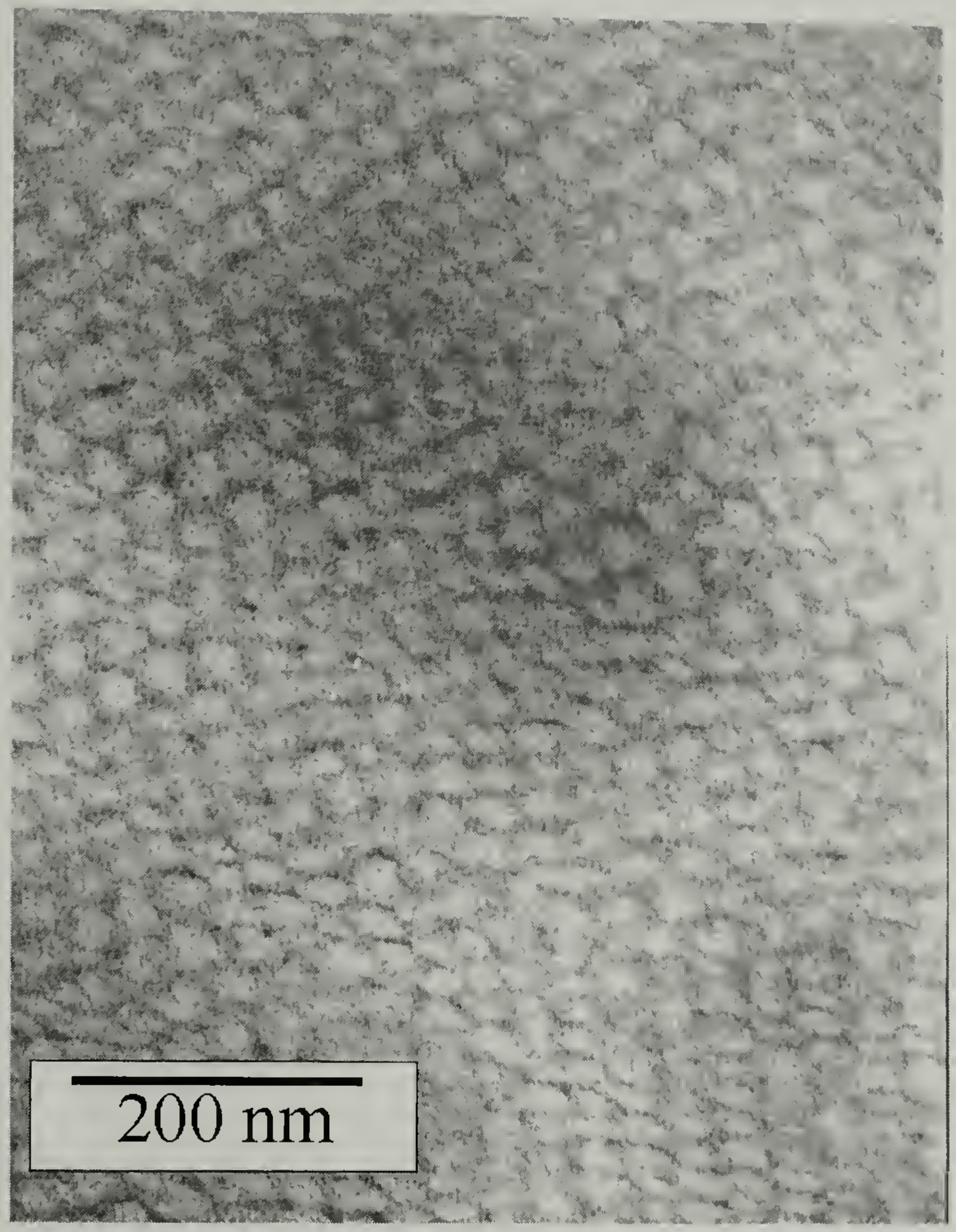

Figure 2.15. The schematic and the TEM micrograph when microtome is parallel to $\mathrm{XY}$ plane. 


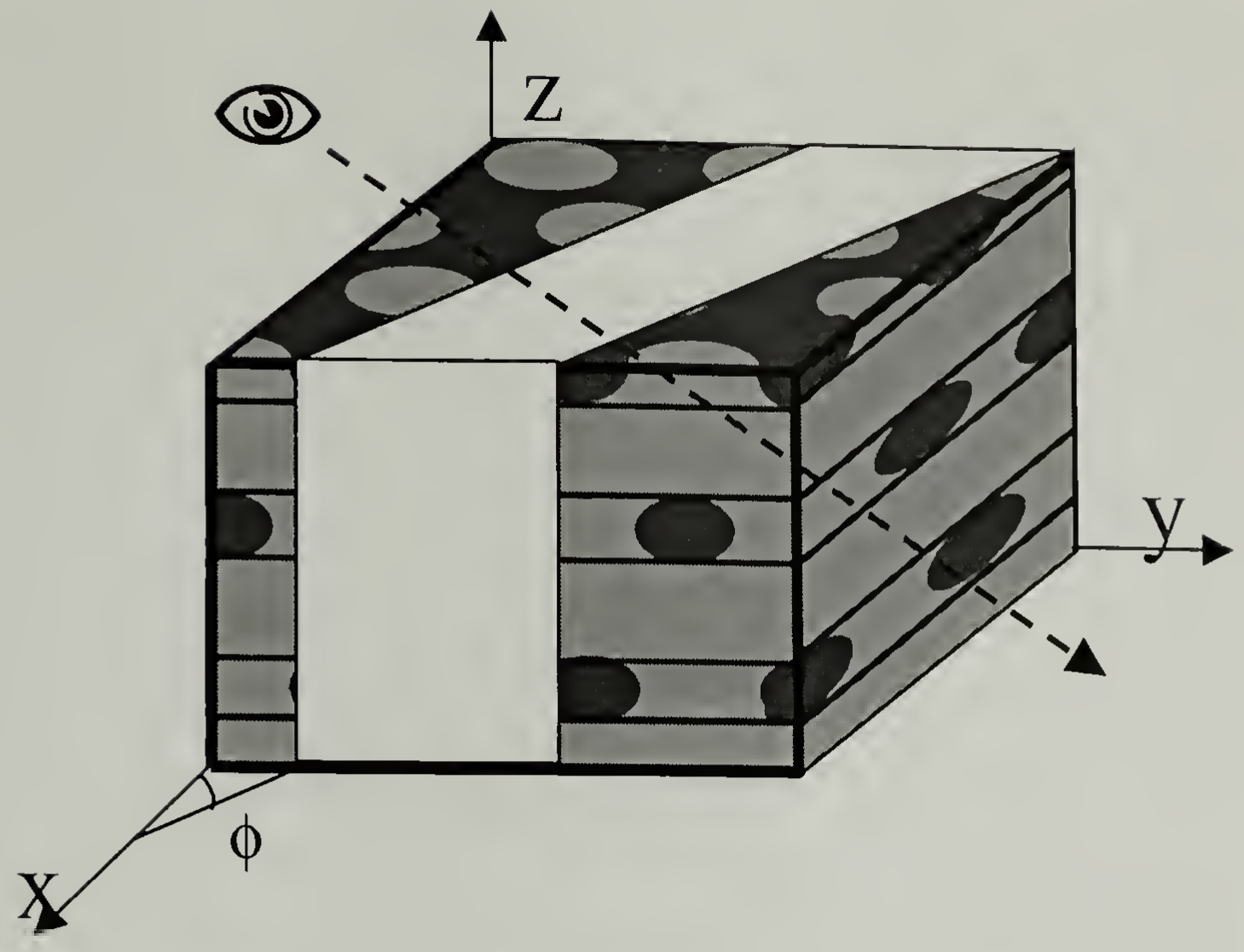

(D)

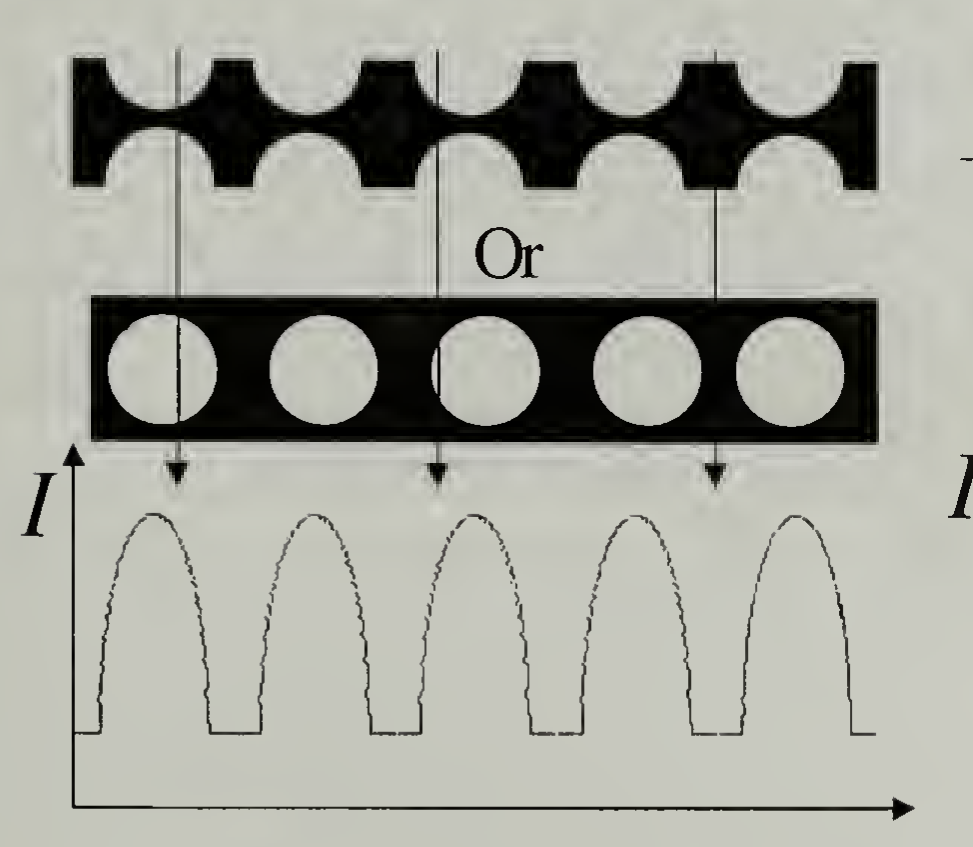

(D)
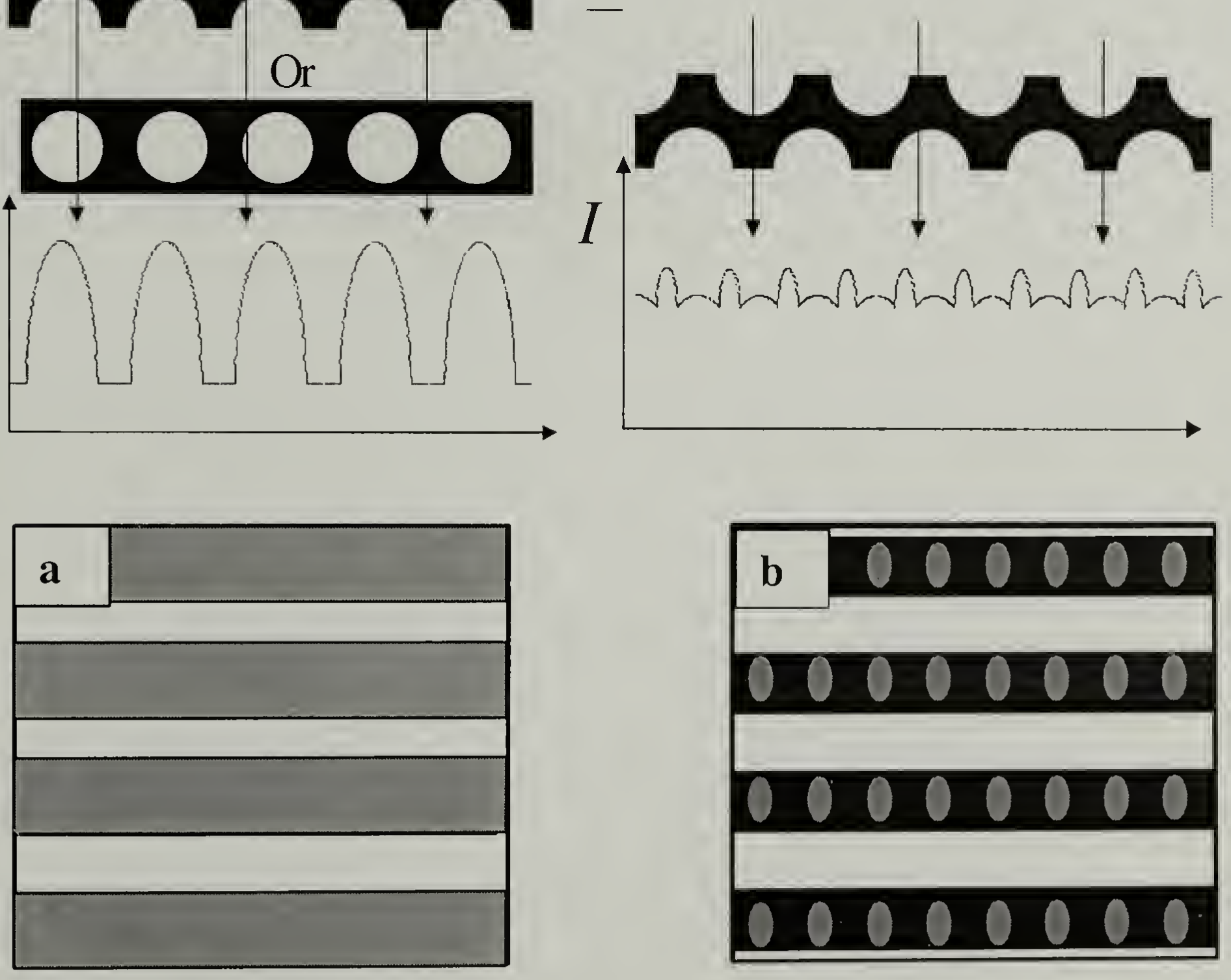

\begin{tabular}{|l|l|llllll|}
\hline$b$ & 0 & 0 & 0 & 0 & 0 & 0 \\
\hline 0 & 0 & 0 & 0 & 0 & 0 & 0 & 0 \\
\hline 0 & 0 & 0 & 0 & 0 & 0 & 0 & 0 \\
\hline 0 & 0 & 0 & 0 & 0 & 0 & 0 & 0 \\
\hline
\end{tabular}



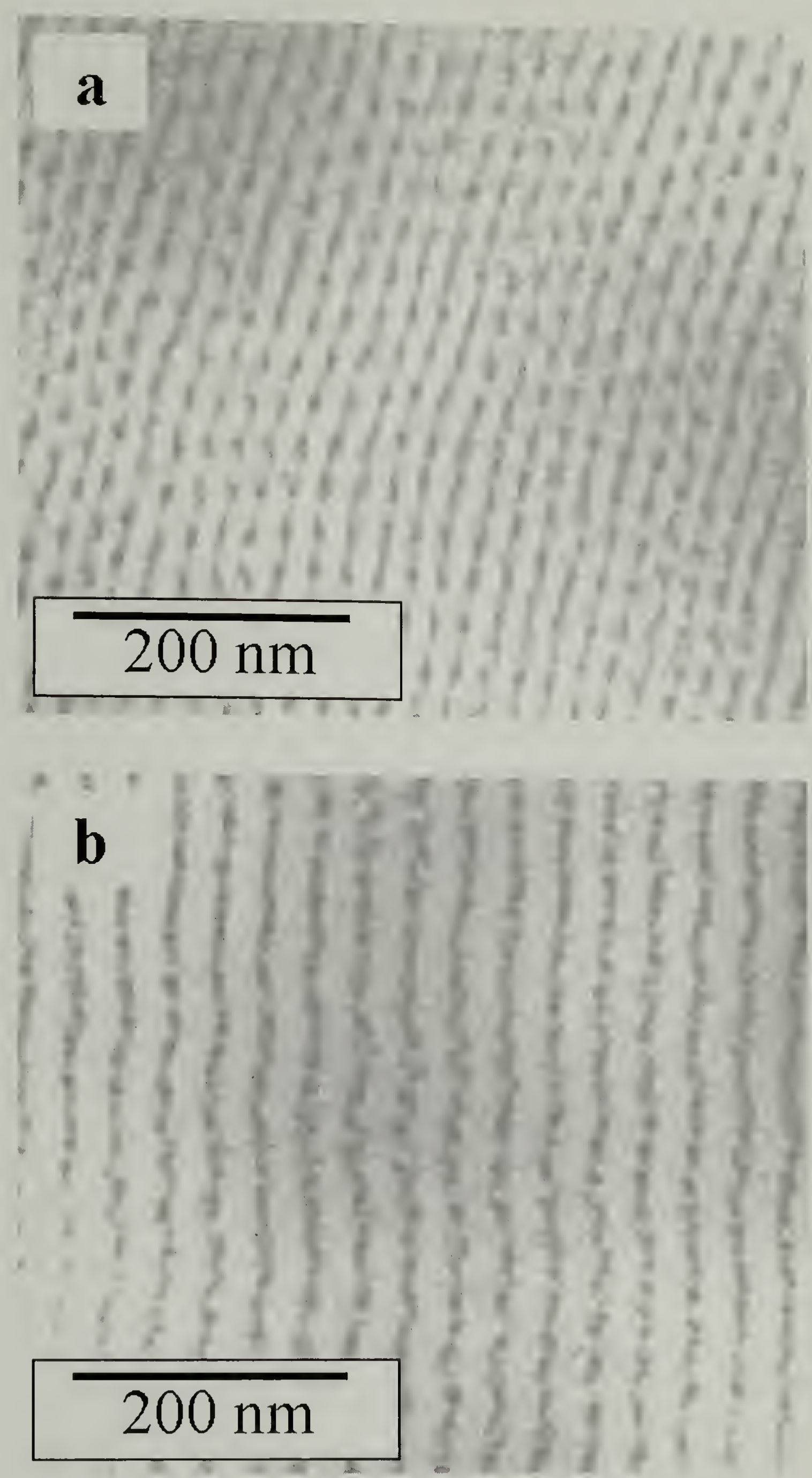

Figure 2.16. The schematic and the TEM micrograph when microtome is perpendicular to XY plane. 


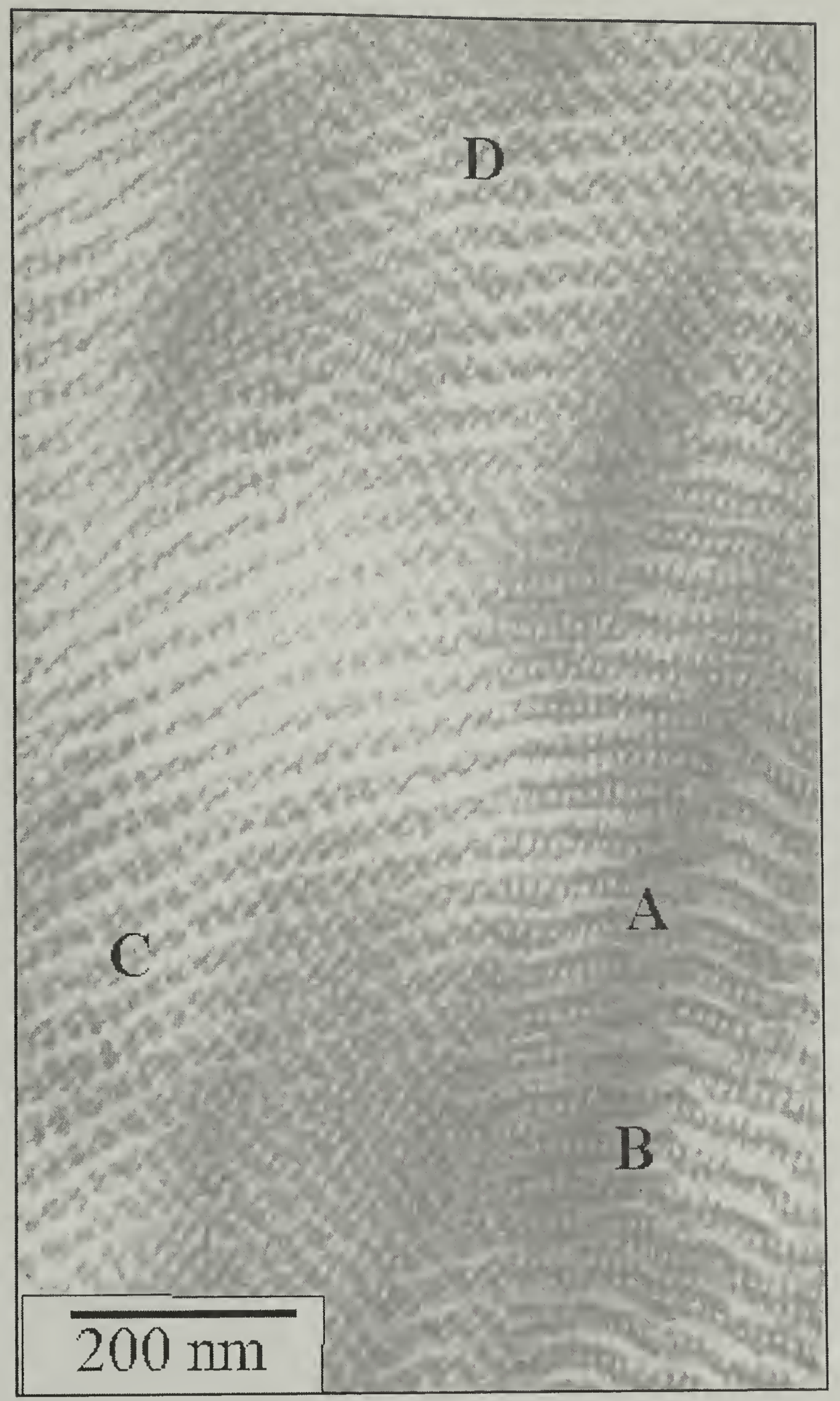

Figure 2.17. The schematic and the TEM micrograph when microtome direction has an angle with XY plane. 


\section{CHAPTER 3}

\section{MORPHOLOGY BEHAVIOR OF X SHAPED SINGLE GRAPHED BLOCK \\ COPOLYMER / HOMOPOLYMER BLENDS}

\subsection{Abstract}

The morphological behavior of blends of architecturally symmetric, yet nonlinear, $\mathrm{I}_{2} \mathrm{~S}_{2}$ miktoarm star block copolymers and homopolymers was investigated using transmission electron microscopy and small angle X-ray scattering. The $\mathrm{I}_{2} \mathrm{~S}_{2}$ block copolymers have two low polydispersity polyisoprene (PI) arms and two low polydispersity polystyrene (PS) arms joined at a common junction point. Previously reported studies of linear diblock copolymer / homopolymer blends showed that the morphology transitions occur at about the same volume fractions as in pure linear diblock copolymers. However, the $\mathrm{I}_{2} \mathrm{~S}_{2}$ block copolymer / homopolymer blends in this study displayed strong shifts in the volume fractions of morphology transitions away from the compositions in which these transitions occur in the pure block copolymer. One of the blend samples formed extremely large and well ordered single crystals of the gyroid morphology. SAXS from these single crystals produces as many as 132 diffraction peaks in a single diffraction pattern. Analysis of this data provides the best evidence to date of the $I a \overline{3} d$ symmetry and Gyroid structure. 


\subsection{Introduction}

The effects of non-linear block copolymer molecular architecture on morphology has been studied by various groups using well defined, model materials. ${ }^{2-}$ 5,8-10,12,44,87-90,99-101 The present study investigates the effects of non-linear symmetric molecular architecture on the morphology of block copolymer / homopolymer blends using $\mathrm{I}_{2} \mathrm{~S}_{2}$ miktoarm star block copolymers. As illustrated in Figure 3.1, $\mathrm{I}_{2} \mathrm{~S}_{2}$ miktoarm stars have two polyisoprene (PI) arms and two polystyrene (PS) arms linked at a common junction point. The morphologies of neat microphase-separated $\mathrm{I}_{2} \mathrm{~S}_{2}$ block copolymers have been studied by Beyer, Gido et. al. ${ }^{9}$ The results of this previous study are plotted in Figure 3.2, on a theoretical morphology diagram for miktoarm stars, calculated by Milner. ${ }^{11}$ For miktoarm stars of type $\mathrm{A}_{\mathrm{n}} \mathrm{B}_{\mathrm{m}}$, this theory predicts morphology as a function of $\mathrm{B}$ component volume fraction, $\phi_{\mathrm{B}}$, and a molecular asymmetry parameter, $\varepsilon=\left(n_{\mathrm{A}} / n_{\mathrm{B}}\right)\left(l_{\mathrm{A}} / l_{\mathrm{B}}\right)^{1 / 2}$. Here, $n_{\mathrm{A}}$ and $n_{\mathrm{B}}$ are the numbers of arms of block materials $\mathrm{A}$ and $\mathrm{B}$, and $l_{\mathrm{i}}=\left(\mathrm{V}_{\mathrm{i}} / \mathrm{R}_{\mathrm{i}}{ }^{2}\right)=v_{\mathrm{i}} / \mathrm{b}_{\mathrm{i}}{ }^{2} . \mathrm{V}_{\mathrm{i}}$ and $\mathrm{R}_{\mathrm{i}}$ are volume and radius of gyration of one arm of polymer $i$, while $v_{\mathrm{i}}$ is the segmental volume and $\mathrm{b}_{\mathrm{i}}$ the statistical segment length of component $i$. The morphologies observed for the $\mathrm{A}_{2} \mathrm{~B}_{2}$ materials showed general agreement with the composition ranges predicted by the theory. However, for some architecturally asymmetric samples, the theory overestimated the degree of shift in the order-order transitions. Studies on $\mathrm{S}_{2} \mathrm{I}, \mathrm{I}_{2} \mathrm{~S}, \mathrm{I}_{3} \mathrm{~S}$, and $\mathrm{I}_{5} \mathrm{~S}$ showed qualitative agreement but quantitative disagreement. ${ }^{7-9,87,89,90,102}$ Since the $\mathrm{I}_{2} \mathrm{~S}_{2}$ samples have a symmetric molecular architecture, the conformational asymmetry between PI (the A component) and PS (the B component) is the only contributor to the molecular asymmetry, resulting in an asymmctry value of $\varepsilon \approx 0.85$. Sincc the $\varepsilon$ value is smaller 
than 1 , we have extended the morphology diagram to include $\varepsilon$ values from $1 / 2$ to 1 . The morphology diagram is calculated for pure miktoarm star block copolymers. In this study, we will use it as a guide to thinking about the effects of homopolymer blending on the morphological transitions of $\mathrm{I}_{2} \mathrm{~S}_{2}$ block copolymer systems.

Blends of linear diblock copolymers with homopolymer as well as blends of linear multi block copolymers with homopolymer have been extensively studied. ${ }^{13-25}$ Previously reported results for linear diblock copolymer / homopolymer blends showed that the order-order transitions (OOTs) occur at about the same volume fractions as in pure linear diblock copolymers. The OOT occurs at the same volume fraction regardless of the direction from which it is approached, i.e. blending homopolymer A with a diblock which forms A cylinders in a B matrix to push it toward lamella or blending B homopolymer with a lamellar diblock to push it back toward cylinders. ${ }^{17,18,20}$ However, for architecturally asymmetric graft copolymers, blending homopolymer into the one arm side or into the multi-arm side of the interface may produce different morphological results. In a recent study by Yang, Gido et.al. ${ }^{54} \mathrm{~A}$ shift in OOT volume fraction is observed when blending homopolymer with the twoarm side of an architecturally asymmetric $\mathrm{I}_{2} \mathrm{~S}$ block copolymer. The current study involves block copolymer materials that, although architecturally symmetric, also have nonlinear molecular structures.

In the present investigation, low molecular weight homopolymers of polystyrene (hPS) or homopolymer polyisoprene (hPI) are blended with $\mathrm{I}_{2} \mathrm{~S}_{2}$ block copolymers to push the morphology transitions. Table 3.1 lists the molecular 
characteristics (molecular weights, volume fractions, polydispersities) and morphologies of the neat $\mathrm{I}_{2} \mathrm{~S}_{2}$ block copolymers used in this study as well as the molecular weights and polydispersites of the homopolymers. We use low molecular weight (MW) homopolymer in all the blend systems so that the distribution of the homopolymer within the block copolymer domains of like material is relatively uniform. ${ }^{14,19,91,95}$

A microphase separated, bicontinuous, block copolymer structure with a cubic symmetry was identified as ordered bicontinuous double diamond (OBDD) with Pn3m symmetry by Thomas and coworkers, ${ }^{44}$ and the Hashimoto group,${ }^{45}$ in the mid-1980's. In 1994, Thomas and co-workers ${ }^{46}$ and Bates and co-workers ${ }^{38}$ amended the structure of the cubic bicontinuous morphology, proposing that it was actually a gyroid structure with $I a \overline{3} d$ symmetry. SAXS evidence for the gyroid structure was provided by powder patterns with up to 13 reflections, ${ }^{38}$ and single crystal scattering patterns with up to 10 reflections. ${ }^{37-39,47}$ In this study, we obtained a gyroid sample with such an amazing long range order that up to 132 unique reflections were observed in a single SAXS pattern, and 152 reflections were observed overall. These scattering patterns provide the best evidence for gyroid morphology observed so far.

\subsection{Experimental}

\subsubsection{Synthesis}

A series of five $A_{2} B_{2}$ miktoarm stars was synthesized using methods similar to those reported by Iatrou and Hadjichristidis. ${ }^{101}$ In the present work, however, 
stoichiometric quantities of tetrachlorosilane were used, eliminating the need to remove excess silane. All manipulations were performed under high vacuum in all-glass, $n$-butyllithium-washed, benzene-rinsed reactors with breakseals for introduction of reactants and constriction seal-offs for sampling of intermediate products. Polystyryllithium and polyisoprenyllithium were separately prepared in benzene using sec-butyllithium initiator. In the linking reactor, tetrachlorosilane in benzene was chilled in an ice bath for ten minutes. Polystyryllithium was then added to the chlorosilane from a side flask at room temperature; more than one equivalent was added quickly with stirring and then the content was stirred at $0^{\circ} \mathrm{C}$ for twenty minutes. During this time the reduced vapor pressure in the reactor prevented transfer of the volatile silane to the side flask. The reactor was then warmed to room temperature and small incremental additions of polystyryllithium were made from the same side flask until essentially pure coupled product, as determined by size-exclusion chromatography (SEC) on sampled aliquots, was obtained. Steric effects and the slow addition of polystyryllithium prevented the formation of detectable amounts of three or four arm PS stars. Addition of more than twofold polyisoprenyllithium and subsequent fractionation yielded the $\mathrm{A}_{2} \mathrm{~B}_{2}$ copolymer samples.

\subsubsection{Molecular Characterization}

The molecular characteristics of the arms and fractionated miktoarm stars are reported in Table 3.1. SEC was performed in tetrahydrofuran (THF) at $30^{\circ} \mathrm{C}$ using a Waters Model 510 pump, Waters Model 410 differential refractometer, and ultrastyragel columns with a continuous porosity range from $10^{6}$ to $10^{3} \AA$. For calculation of $M_{n}$ values, calibration with absolute standards was performed. 
Membrane osmometry (MO) using a Jupiter Model 231 recording membrane osmometer was condueted in toluene distilled from $\mathrm{CaH}_{2}$. Low-angle light scattering (LALLS) was performed in THF distilled from sodium, using a Chromatix KMX-6 instrument operating at $633 \mathrm{~nm}$. Matrix-assisted laser desorption/ionization time-offlight mass spectroscopy (MALDI/TOF/MS) was performed in trans-retinoic acid with silver trifluoroacetate using a Perseptive Biosystems Voyager Elite DE instrument. Proton nuclear magnetic resonance ( $\left.{ }^{1} \mathrm{H}-\mathrm{NMR}\right)$ was earried out in $\mathrm{CDCl}_{3}$ using a $\mathrm{Bruker}$ $300 \mathrm{MHz}$ instrument. Ultraviolet spectroseopy (UV) was conducted in THF using a Waters Alliance 2690 separations module and Waters 996 photodiode array detector.

\subsubsection{Morphological Characterization}

The blends were produced by eo-dissolving $\mathrm{I}_{2} \mathrm{~S}_{2}$ block copolymer and homopolymer, in the appropriate proportions, in toluene, a non-preferential solvent. Solid film approximately $1 \mathrm{~mm}$ thick was slowly east at room temperature from these toluene solutions over a period of 4 weeks. Solvent evaporation was slowed by covering the samples with a erystallizing dish in order to promote the formation of well-ordered struetures. After solidification of the samples, residual toluene was removed by placing the samples under vacuum at ambient temperature for another week. The samples were then annealed for one week under high vacuum at $150^{\circ} \mathrm{C}$ in order to further promote ordering. Finally, these blend samples were cooled under vacuum to room temperature over a period of several hours.

Sample morphologies were characterized using a combination of transmission electron microscopy (TEM) and small-angle X-ray scattering (SAXS). To prepare thin 
sections for microscopy, a Leica Ultracut UCT microtome equipped with a Leica EM FCS cryogenic sample chamber operated at $-110^{\circ} \mathrm{C}$ was used to cut sections approximately $500 \AA$ in thickness. The sections were collected on TEM grids and stained for four hours in $\mathrm{OsO}_{4}$ vapor. The PI microdomains are preferentially stained by $\mathrm{OsO}_{4}$, rendering them dark relative to PS microdomains due to mass-thickness contrast in the TEM. A JEOL 100CX TEM, operated at an accelerating voltage of 100 $\mathrm{kV}$, was used to image the stained sections. SAXS data were collected at the Advanced Polymers Beamline (X27C), located at the National Synchrotron Light Source at Brookhaven National Labs (BNL), Upton, NY. Two-dimensional scattering patterns were collected on Fujitsu image plates, then read by a Fujitsu BAS 2000 image plate reader. Custom software at BNL was used to subtract background noise and perform circular averaging. Data were collected for a wavelength of $1.307 \AA$ and a camera length of $1410 \mathrm{~mm}$.

Four different series of blends were prepared and their morphologies were examined. Blends are designated as either IXn-m or SXn-m. The first character, I or $S$, indicates that the homopolymer used in the blend series is homopolyisoprene (I) or homopolystyrene (S). The second character, $\mathrm{X}$, indicates that the block copolymer used is an $\mathrm{X}$ shaped $\mathrm{I}_{2} \mathrm{~S}_{2}$ miktoarm star block copolymer. The first number $\mathrm{n}$ indicates the PS volume fraction of the $\mathrm{I}_{2} \mathrm{~S}_{2}$ graft copolymer on which the blend series is based. The last number, $\mathrm{m}$, indicates the PS volume fraction of the overall blend after addition of homopolymer. The compositions of all the blends studied in this paper are given in Table2. 
SX14 series:

The pure $\mathrm{I}_{2} \mathrm{~S}_{2}-14$, with polystyrene volume fraction $\Phi_{\mathrm{PS}}=14 \%$, forms hexagonally packed PS cylinders with reported 100 spacing of $27.7 \pm 0.5 \mathrm{~nm}{ }^{9}$ The SAXS data for the SX14 blend series are shown in Figure 3.3. The series of $q_{n} / q^{*}$ ratios are the scattering vectors of all the Bragg peaks observed divided by the scattering vector of the primary reflection. The $q_{n} / q^{*}$ ratios of $1, \sqrt{3}, \sqrt{4}$ and $\sqrt{7}$ indicate that SX14-20, SX14-25, SX14-29, SX14-34, and SX14-38 form cylindrical morphologies. The cylinder (100) spacing initially decreases from $\mathrm{I}_{2} \mathrm{~S}_{2}-14$ to SX14-20; and then increases with increasing homopolymer concentration from SX14-20 to SX1438. This behavior is similar to previous published results on linear block copolymer / homopolymer blends. ${ }^{19}$ The scattering vectors of the peaks occurring at integral multiples of $q^{*}$ indicates that SX14-44 forms a lamellar morphology. TEM observation (data not shown) confirmed the morphologies observed in SAXS. Therefore, as the homopolymer concentration increases, the morphology of the blends changes from cylinder to lamella at an overall PS volume fraction between 0.38 and 0.44 . No gyroid morphology was observed in this blend series. The morphologies of the SX14 series are mapped onto the morphology diagram in Figure 3.4.

\section{SX28 series}

The pure four-arm star $\mathrm{I}_{2} \mathrm{~S}_{2}-28$, with polystyrene volume fraction $\Phi_{\mathrm{PS}}=28 \%$, forms hexagonally packed PS cylinders with a reported cylinder spacing of $31.9 \pm 0.5$ 
nm. ${ }^{9}$ The SAXS data for the SX28 blend series are shown in Figure 3.5. The SAXS data indicate that the first blends sample, SX28-36, which has $q_{1} / q^{*}$ ratio of $1, \sqrt{3}, \sqrt{4}$, $\sqrt{7}, \sqrt{9}$, and $\sqrt{12}$, forms a cylindrical morphology. The next three blends, SX28-39, SX28-43, and SX28-46 form gyroid morphologies. For SX28-39 and SX28-43, $\mathrm{q}_{\mathrm{n}} / \mathrm{q}^{*}=$ $1, \frac{\sqrt{8}}{\sqrt{6}}, \frac{\sqrt{14}}{\sqrt{6}}, \frac{\sqrt{20}}{\sqrt{6}}, \frac{\sqrt{24}}{\sqrt{6}}, \frac{\sqrt{26}}{\sqrt{6}}, \frac{\sqrt{32}}{\sqrt{6}}, \frac{\sqrt{38}}{\sqrt{6}}, \frac{\sqrt{42}}{\sqrt{6}}, \frac{\sqrt{50}}{\sqrt{6}}$; for $\mathrm{SX} 28-46, \mathrm{q}_{\mathrm{n}} / \mathrm{q}^{*}=1$, $\frac{\sqrt{8}}{\sqrt{6}}, \frac{\sqrt{14}}{\sqrt{6}}, \frac{\sqrt{20}}{\sqrt{6}}, \frac{\sqrt{24}}{\sqrt{6}}, \frac{\sqrt{38}}{\sqrt{6}}, \frac{\sqrt{46}}{\sqrt{6}} \cdot \frac{\sqrt{52}}{\sqrt{6}}$, The last blend in the series SX28-49, which has integral $q_{11} / q^{*}$ ratios, forms lamella. From $\mathrm{I}_{2} \mathrm{~S}_{2}-28$ to SX28-36 in the cylinder morphology and from SX28-39 to SX28-47 in the gyroid morphology the domain spacing is found to increase with increasing homopolymer concentration. As shown in Figure 3.6, the TEM images confirm the morphologies observed in SAXS. In the SX28 blend series, the cylinder to gyroid morphology transition occurs at the PS overall volume fraction between $36 \%$ to $39 \%$, and the gyroid to lamella morphology transition occurs at the PS overall volume fraction between $46 \%$ to $49 \%$. The morphologies of the SX28 series are mapped onto the theoretical morphology diagram in Figure 3.7.

\section{IX68 series}

The pure four-arm star $\mathrm{I}_{2} \mathrm{~S}_{2}-68$, with a polystyrene volume fraction $\Phi_{\mathrm{PS}}=68 \%$, forms hexagonally packed PI cylinders with reported 100 spacing of $32.5 \pm 0.5 \mathrm{~nm}$. . The SAXS data of this blend series are shown in Figure 3.8. The $q_{n} / q^{*}$ ratios of $1, \sqrt{3}$, $\sqrt{4}, \sqrt{7}, \sqrt{9}$, and $\sqrt{12} \ldots$ indicate that IX68-65 and IX68-61 form cylindrical 
morphologies with PI in the cores of the cylinders. The $q_{n} / q^{*}$ ratios of $1, \frac{\sqrt{8}}{\sqrt{6}}, \frac{\sqrt{14}}{\sqrt{6}}$, $\frac{\sqrt{20}}{\sqrt{6}}, \frac{\sqrt{24}}{\sqrt{6}}, \frac{\sqrt{32}}{\sqrt{6}}, \frac{\sqrt{40}}{\sqrt{6}}$ and $\frac{\sqrt{52}}{\sqrt{6}}$ indicate that IX68-58 forms a gyroid morphology; and the integral $q_{n} / q^{*}$ ratios indicate that IX68-53, IX68-49, IX68-41, and IX68-41 form lamellar morphologies. The morphologies observed in SAXS were confirmed by TEM, images are shown in Figure 3.9. Interestingly, the last blend in this series, IX6837, forms a cylindrical morphology with PS in the cylinder cores, i.e. the inverse of the pure $\mathrm{I}_{2} \mathrm{~S}_{2}-68$. The observed morphologies in the IX68 series are mapped onto the theoretical calculated morphology diagram in Figure 3.10. In these blends, the transition from PI cylinders to lamella occurs at an overall PS volume fraction between 0.61 and 0.58 . The transition from gyroid to lamella occurs at an overall PS volume fraction between 0.58 and 0.53 ; and the transition from lamella to PS cylinders occurs at an overall PS volume fraction between 0.41 and 0.37 . Within the same morphology, i.e. from pure $\mathrm{I}_{2} \mathrm{~S}_{2}-68$ to IX68-61 and from IX68-53 to IX68-41, the domain spacing increases with increasing homopolymer concentration

\section{IX35 series}

The pure four-arm star block copolymer $\mathrm{I}_{2} \mathrm{~S}_{2}-35$, with polystyrene volume fraction $\Phi_{\mathrm{PS}}=35 \%$, forms a lamellar morphology with a reported lamella spacing of $36.3 \pm 0.5 \mathrm{~nm} .{ }^{9}$ The SAXS data from the IX35 blend series are shown in Figure 3.11. Blend sample IX35-33, which has integral $q_{n} / q^{*}$ ratios, form lamellar morphology. The 
$q_{n} / q^{*}$ ratios of $1, \frac{\sqrt{8}}{\sqrt{6}}, \frac{\sqrt{20}}{\sqrt{6}}, \frac{\sqrt{22}}{\sqrt{6}}, \frac{\sqrt{32}}{\sqrt{6}}, \frac{\sqrt{38}}{\sqrt{6}}, \frac{\sqrt{46}}{\sqrt{6}} \frac{\sqrt{48}}{\sqrt{6}}, \frac{\sqrt{50}}{\sqrt{6}}, \frac{\sqrt{62}}{\sqrt{6}}, \frac{\sqrt{72}}{\sqrt{6}}$, $\frac{\sqrt{86}}{\sqrt{6}} \ldots$ indicate that IX35-30 forms the gyroid morphology. The $q_{n} / q^{*}$ ratios of $1, \sqrt{3}$, $\sqrt{4}$, and $\sqrt{7} \ldots$ indicate that IX35-27, IX35-24, IX35-20, and IX35-17 all form cylindrical morphologies. In the IX35 blend series, the lamella to gyroid morphology transition occurs at an overall PS volume fraction between $33 \%$ and $30 \%$. The gyroid to cylinder transition occurs at an overall PS volume fraction between $30 \%$ and $27 \%$. The cylinder spacing increases with increasing homopolymer concentration from IX3527 to IX35-17. IX35-31 formed an extremely well ordered single crystal like gyroid structure. A two-dimensional SAXS pattern of this single crystal is shown in Figure 3.12a. The X-ray beam is perpendicular to the surface of the $2 \mathrm{~mm}$ thick IX35-3 I sample. Figure $3.12 \mathrm{~b}$ gives the indexing of this pattern. From this single SAXS pattern, 132 unique reflections are observed. The relative intensities of these reflections are listed in Table 3. Combined with another SAXS pattern from a different zone, I60 total unique diffraction peaks were observed. The observed reflections are listed in Table 4 to compare with the permitted reflections for $I a \overline{3} d$ symmetry. All the observed reflections are permitted for $I a \overline{3} d$ symmetry; and most of the permitted reflections for $I a \overline{3} d$ are observed. The near perfect long-range order of this sample is also supported by TEM observations. A TEM micrograph of IX35-3I is shown in Figure 3.13. Other blends in the IX35 series were also studied by TEM. The TEM observation (image not shown here) supports the morphologies observed though SAXS. 
The morphologies of IX35 series are mapped onto the morphology diagram in Figure 3.14 .

\subsection{Discussion}

In linear diblock copolymer / low molecular weight homopolymer blends the morphology transitions occur at roughly the same volume fraction regions as those of pure diblock copolymers. ${ }^{17,18,20}$ The morphology transitions in the $\mathrm{I}_{2} \mathrm{~S}_{2}$ block copolymer / homopolymer blends exhibit complex behaviors. The gyroid to lamella transition of linear diblock copolymers occurs at volume fraction of about 0.33 and 0.66. According to the morphology diagram, architecturally symmetric $\mathrm{A}_{2} \mathrm{~B}_{2}$ should be morphologically similar to linear $\mathrm{AB}$ diblocks. Considering the conformational asymmetry between PS and PI in the $\mathrm{I}_{2} \mathrm{~S}_{2}$ systems, the gyroid to lamella transitions for pure $\mathrm{I}_{2} \mathrm{~S}_{2}$ block copolymers occurs at PS volume fractions around 0.28 and 0.63 . In the IX35 blend series, based on the lamella forming $\mathrm{I}_{2} \mathrm{~S}_{2}-35$, a transition to Gyroid and then to PS cylinders occurs over a volume fraction range $(0.33-0.27)$ which is similar to that predicted for these transitions in pure $\mathrm{I}_{2} \mathrm{~S}_{2}$. This behavior is similar to that of linear diblock copolymer / homopolymer blends. However, in the SX14 and SX28 series, the transitions from cylinder to lamella (SX14) and from gyroid to lamella (SX28) occur at PS volume fractions between 0.38 and 0.44 and between 0.46 and 0.49 , respectively. These are strong shifts from the $28 \%$ predicted for pure $\mathrm{I}_{2} \mathrm{~S}_{2}$ block copolymers. Thus there is a splitting of the cylinder-gyroid-lamellar transitions depending upon whether one is blending in PS homopolymer (SX14 and SX28 series) or PI homopolymer (IX35 series). Similar splitting was previously observed in blends of homopolymer with 
asymmetric $\mathrm{I}_{2} \mathrm{~S}$ miktoarm star block copolymers where it was attributed to the effect of molecular asymmetry on the differing ability of homopolymer to penetrate the grafted brushes of block copolymer arms on the two arm vs. one arm side of the interface. ${ }^{54}$ In the current study, splitting of OOTs is observed even though the $\mathrm{I}_{2} \mathrm{~S}_{2}$ miktoarm stars have two arms per molecule on both sides of the interface. Due to the shifts in OOT volume fractions, morphologies can occurs at quite different volume fraction ranges as that of linear diblock systems. For example, the SX28-43, SX28-46, IX68-58 was observed to form gyroid morphologies at $43 \%, 46 \%$, and $58 \%$ polystyrene volume fractions, which are well into the lamella region in linear diblock system; SX14-38 and IX68-61 from cylinder morphology at 38\% and 61\% PS volume fraction, which are also in the lamella region in linear diblock systems. This provides another parameter to tailor the morphologies block copolymer systems.

Inverted phases bring up by solvents was demonstrated by Sadron and Gallot for a cylindrical diblock copolymer and recently by Lodge and coworkers for a gyroid diblock copolymer. ${ }^{103}$ However, morphology inversions that result from homopolymers have not been reported. Inside-out or inverted morphologies require the short block of the copolymer to swell to such an extent that its effective volume is larger than that of the unswollen long block of the copolymer. The swelling of the short block is promoted by the favorable entropy of mixing. In reported linear diblock copolymer / homopolymer blends, starting from cylinder forming diblock and blend homopolymer with the minority component, Winey, Thomas and Fetters ${ }^{17}$ showed that as the homopolymer concentration increase, the cylinder morphology transform into lamella and then into macrophase scparation. In that study, the homopolymer is PS, the 
molecular weight of the hPS is $5.900 \mathrm{~g} / \mathrm{mol}$ and the PS block molecular weights of the block copolymers are around 13,000 $\mathrm{g} / \mathrm{mol}$, which is about 2 times that MW of homopolymer. Blend sample IX68-37, which contains 44\% homopolyisoprene and $56 \% \mathrm{I}_{2} \mathrm{~S}_{2}-68$, forms cylinder morphology with PS form the core of the cylinders, which is the inverse of pure $\mathrm{I}_{2} \mathrm{~S}_{2}-68$. Even with $44 \%$ low $\mathrm{MW}$ homopolymer present in the matrix (PI), the PS cylinders still form good long-range order, as indicated by the SAXS data. The molecular weight of the hPI is $1,200 \mathrm{~g} / \mathrm{mol}$ and the PI arm of $\mathrm{I}_{2} \mathrm{~S}_{2}-68$ $12,900 \mathrm{~g} / \mathrm{mol}$, which is about 10 times of the MW of homopolymer. These results indicates a greater solubility range for selective solvent molecules and for low molecular weight homopolymer than for higher MW homopolymers, and lower MW homopolymer maintains the long range order of the system.

IX35-30 and IX68-58 form extremely well ordered gyroid morphology. The single crystal SAXS pattern indicates the degree of ordering of this sample. Perfect single crystals are usually observed in atom and molecular level self-assemblies with each consisting of materials perfectly the same. In nano-scale self-assemblies, the repeat unit usually consists of hundreds to tens of thousands of atoms and the component molecules are not necessarily identical. Fluctuations and defects lead to imperfections and prevent the formation of large single crystal. All the cylinder or lamella blend samples are processed at the same conditions, but none of them have nearly the same degree of ordering as the Gyroid samples. This observation implies the defects structure in the interpenetration network of Gyroid have relatively higher energies or kinetically easier to eliminate than that of lamella and cylinder morphologies. These experimental results showed an example that the non-perfect 
block copolymer systems can form near-perfect structures in a large area at certain compositions and process conditions.

\subsection{Conclusions}

Unlike linear diblock copolymer / homopolymer blend systems, shifts in OOT volume fraction is observed in the architecturally symmetry yet non-linear $\mathrm{A}_{2} \mathrm{~B}_{2}$ block copolymer / homopolymer blends series. In IX68 blend series, morphology inversion is observed at high homopolymer concentration. "Gyroid Single crystal" was obtained though slow solvent casting and annealing. The SAXS of the sample provides the best evidence for gyroid morphology so far. 

Table 3.1. Molecular characterization of the $\mathrm{I}_{2} \mathrm{~S}_{2}$ block copolymer and the PS and
PI homopolymers.
${ }^{a}$ from SEC
${ }^{b}$ from membrane osmometry

'Data from reference.

\begin{tabular}{|l|c|c|c|c|c|c|c|}
\hline $\begin{array}{l}\text { Sample } \\
\text { Name }\end{array}$ & $\begin{array}{c}{ }^{a} \mathrm{M}_{\mathrm{n}}(\mathrm{PS} \\
\mathrm{arm}) \\
(\mathrm{g} / \mathrm{mol})\end{array}$ & $\begin{array}{c}{ }^{\mathrm{a}} \mathrm{M}_{\mathrm{n}}(\mathrm{PI} \\
\mathrm{arm}) \\
(\mathrm{g} / \mathrm{mol})\end{array}$ & $\begin{array}{c}{ }^{\mathrm{b}} \mathrm{M}_{\mathrm{n}} \\
(\text { total })\end{array}$ & ${ }^{\mathrm{a}} \mathrm{M}_{\mathrm{w}} / \mathrm{M}_{\mathrm{n}}$ & $\begin{array}{c}{ }^{\mathrm{c}} \mathrm{V}_{\mathrm{ol}} \% \\
\mathrm{PS}\end{array}$ & ${ }^{\mathrm{c}} \mathrm{q}^{*}\left(\AA^{-1}\right)$ & ${ }^{\mathrm{c}}$ Morphology \\
\hline $\mathrm{I}_{2} \mathrm{~S}_{2}-14$ & 9,080 & 41,000 & 106,000 & 1.01 & 14 & 0.0227 & cylinder \\
\hline $\mathrm{I}_{2} \mathrm{~S}_{2}-28$ & 10,400 & 33,000 & 104,000 & 1.01 & 28 & 0.0197 & cylinder \\
\hline $\mathrm{I}_{2} \mathrm{~S}_{2}-35$ & 20,500 & 30,800 & 99,000 & 1.03 & 35 & 0.0173 & lamella \\
\hline $\mathrm{I}_{2} \mathrm{~S}_{2}-68$ & 37,300 & 12,900 & 104,000 & 1.01 & 68 & 0.0193 & cylinder \\
\hline $\mathrm{hPS}$ & & & 1,050 & 1.13 & & & \\
\hline $\mathrm{hPI}$ & & & 1,020 & 1.17 & & & \\
\hline
\end{tabular}


Table 3.2. Compositions and Morphologies of the blends in the study.

${ }^{\mathrm{a}}$ Calculated utilizing density of PS $=1.04 \mathrm{~g} / \mathrm{ml}$ at $25^{\circ} \mathrm{C}$ and density of PI $=0.91 \mathrm{~g} / \mathrm{ml}$ at $25^{\circ} \mathrm{C}$.

${ }^{\mathrm{b}} \mathrm{q}^{*}=4 \pi / \lambda\left(\sin \theta_{1}\right)$ and $2 \theta_{1}$ is the scattering angle for the lowest angle Bragg peak; corresponds to $\mathrm{d}_{(100)}$ for hexagonal packed cylinders, $\mathrm{d}_{(001)}$ for lamellae, respectively.

\begin{tabular}{|c|c|c|c|c|c|}
\hline $\begin{array}{c}\text { Sample } \\
\text { Name }\end{array}$ & $\begin{array}{c}\text { Weight Fraction } \\
\text { of Homopolymer }\end{array}$ & $\begin{array}{c}\text { Overall Weight } \\
\text { Fraction of PS }\end{array}$ & $\begin{array}{c}\text { a Overall Volume } \\
\text { Fraction of PS }\end{array}$ & Morphology & $\begin{array}{c}\mathrm{b} 2 \pi / \mathrm{q}^{*} \\
( \pm 0.5 \mathrm{~nm})\end{array}$ \\
\hline SX14-20 & 0.07 & 0.22 & 0.20 & Cylinder & 25.5 \\
\hline SX14-25 & 0.13 & 0.27 & 0.25 & Cylinder & 27.9 \\
\hline SX14-29 & 0.18 & 0.32 & 0.29 & Cylinder & 28.3 \\
\hline SX14-34 & 0.25 & 0.37 & 0.34 & Cylinder & 28.7 \\
\hline SX14-38 & 0.30 & 0.41 & 0.38 & Cylinder & 30.4 \\
\hline SX14-44 & 0.37 & 0.47 & 0.44 & Lamella & 31.8 \\
\hline SX28-36 & 0.12 & 0.39 & 0.36 & Cylinder & 33.2 \\
\hline SX28-39 & 0.16 & 0.42 & 0.39 & Gyroid & 32.3 \\
\hline SX28-43 & 0.22 & 0.46 & 0.43 & Gyroid & 33.7 \\
\hline SX28-46 & 0.27 & 0.49 & 0.46 & Gyroid & 35.2 \\
\hline SX28-49 & 0.32 & 0.52 & 0.49 & Lamella & 35.0 \\
\hline IX68-65 & 0.05 & 0.68 & 0.65 & Cylinder & 33.8 \\
\hline IX68-61 & 0.10 & 0.64 & 0.61 & Cylinder & 33.9 \\
\hline IX68-58 & 0.14 & 0.61 & 0.58 & Gyroid & 32.4 \\
\hline IX68-53 & 0.21 & 0.56 & 0.53 & Lamella & 35.2 \\
\hline IX68-49 & 0.26 & 0.53 & 0.49 & Lamella & 36.0 \\
\hline IX68-45 & 0.32 & 0.49 & 0.45 & Lamella & 39.1 \\
\hline IX68-41 & 0.39 & 0.44 & 0.41 & Lamella & 40.3 \\
\hline IX68-37 & 0.44 & 0.40 & 0.37 & Cylinder & 36.0 \\
\hline IX35-33 & 0.07 & 0.36 & 0.33 & Lamella & 39.2 \\
\hline IX35-30 & 0.16 & 0.32 & 0.30 & Gyroid & 37.5 \\
\hline IX35-27 & 0.24 & 0.29 & 0.27 & Cylinder & 37.8 \\
\hline IX35-24 & 0.31 & 0.26 & 0.24 & Cylinder & 38.1 \\
\hline IX35-20 & 0.33 & 0.23 & 0.20 & Cylinder & 38.4 \\
\hline IX35-17 & 0.41 & 0.19 & 0.17 & Cylinder & 38.6 \\
\hline
\end{tabular}


Table 3.3. Observed reflections their intensities. (The intensity of the brightest peak
was set as 1000)

\begin{tabular}{|c|c|c|c|c|c|c|c|}
\hline Index & Intensity & Index & intensity & Index & Intensity & Index & Intensity \\
\hline$(2,2,0)$ & 1000.0 & & & $(-4,-8,2)$ & 106.0 & $(4,8,-2)$ & 11.6 \\
\hline$(4,4,0)$ & 896.6 & & & $(3,-2,3)$ & 280.9 & $(-3,2,-3)$ & 18.7 \\
\hline$(6,6,0)$ & 505.1 & & & $(6,1,3)$ & 31.5 & $(-6,-1,-3)$ & 1.1 \\
\hline$(-2,-2,0)$ & 945.9 & & & $(8,2,2)$ & 22.0 & $(-8,-2,-2)$ & 7.1 \\
\hline$(-4,-4,0)$ & 904.9 & & & $(3,-3,2)$ & 162.7 & $(-3,3,-2)$ & 23.2 \\
\hline$(-6,-6,0)$ & 457.2 & & & $(2,-3,3)$ & 190.7 & $(-2,3,-3)$ & 18.3 \\
\hline$(2,1,1)$ & 112.5 & $(-2,-1,-1)$ & 27.1 & $(-1,-6,3)$ & 80.7 & $(1,6,-3)$ & 6.8 \\
\hline$(4.3 .1)$ & 30.3 & $(-4,-3,-1)$ & 2.4 & $(-2,-8,2)$ & 36.3 & $(2,8,-2)$ & 14.1 \\
\hline$(5,4,1)$ & 113.8 & $(-5,-4,-1)$ & 4.2 & $(5,-2,3)$ & 432.6 & $(-5,2,-3)$ & 46.9 \\
\hline$(6,5,1)$ & 35.1 & $(-6,-5,-1)$ & 3.1 & $(6,-1,3)$ & 279.1 & $(-6,1,-3)$ & 36.4 \\
\hline$(7,6,1)$ & 56.7 & $(-7,-6,-1)$ & 5.5 & $(8,1,3)$ & 28.3 & $(-8,-1,-3)$ & 4.8 \\
\hline$(-1,-2,1)$ & 315.0 & $(1,2,-1)$ & 33.9 & $(2,-5,3)$ & 370.0 & $(-2,5,-3)$ & 45.9 \\
\hline$(-3,-4,1)$ & 145.1 & $(3,4,-1)$ & 8.9 & $(1,-6,3)$ & 218.5 & $(-1,6,-3)$ & 25.9 \\
\hline$(-4,-5,1)$ & 238.4 & $(4,5,-1)$ & 9.6 & $(-1,-8,3)$ & 10.1 & $(1,8,-3)$ & 2.0 \\
\hline$(-5,-6,1)$ & 74.1 & $(5,6,-1)$ & 4.3 & $(4,-4,4)$ & 1052.1 & $(-4,4,-4)$ & 100.7 \\
\hline$(-6,-7,1)$ & 68.6 & $(6,7,-1)$ & 1.7 & $(5,-3,4)$ & 667.2 & $(-5,3,-4)$ & 63.8 \\
\hline$(2,-1,1)$ & 242.2 & $(-2,1,-1)$ & 42.1 & $(6,-2,4)$ & 70.0 & $(-6,2,-4)$ & 5.5 \\
\hline$(5,2,1)$ & 223.7 & $(-5,-2,-1)$ & 23.9 & $(8,0,4)$ & 285.2 & $(-8,0,-4)$ & 24.3 \\
\hline$(6,3,1)$ & 121.4 & $(-6,-3,-1)$ & 19.6 & $(3,-5,4)$ & 644.5 & $(-3,5,-4)$ & 55.9 \\
\hline$(7,4,1)$ & 8.4 & $(-7,-4,-1)$ & 1.1 & $(2,-6,4)$ & 63.7 & $(-2,6,-4)$ & 7.3 \\
\hline$(8,5,1)$ & 32.1 & $(-8,-5,-1)$ & 8.4 & $(0,-8,4)$ & 209.9 & $(0,8,-4)$ & 19.8 \\
\hline$(1,-2,1)$ & 227.7 & $(-1,2,-1)$ & 45.2 & $(7,-3,4)$ & 49.7 & $(-7,3,-4)$ & 3.2 \\
\hline$(-2,-5,1)$ & 191.1 & $(2,5,-1)$ & 29.5 & $(8,-2,4)$ & 90.4 & $(-8,2,-4)$ & 12.5 \\
\hline$(-3,-6,1)$ & 141.7 & $(3,6,-1)$ & 17.4 & $(3,-7,4)$ & 100.1 & $(-3,7,-4)$ & 10.8 \\
\hline$(-4,-7,1)$ & 29.7 & $(4,7,-1)$ & 4.9 & $(2,-8,4)$ & 90.2 & $(-2,8,-4)$ & 14.6 \\
\hline$(-5,-8,1)$ & 64.9 & $(5,8,-1)$ & 9.5 & $(6,-5,5)$ & 544.9 & $(-6,5,-5)$ & 53.0 \\
\hline$(3,-1,2)$ & 17.7 & $(-3,1,-2)$ & 1.1 & $(7,-4,5)$ & 257.1 & $(-7,4,-5)$ & 28.2 \\
\hline$(4,0,2)$ & 883.5 & $(-4,0,-2)$ & 4.9 & $(5,-6,5)$ & 527.7 & $(-5,6,-5)$ & 44.4 \\
\hline$(5,1,2)$ & 392.0 & $(-5,-1,-2)$ & 43.3 & $(4,-7,5)$ & 188.2 & $(-4,7,-5)$ & 22.2 \\
\hline$(7,3,2)$ & 200.3 & $(-7,-3,-2)$ & 9.8 & $(8,-6,6)$ & 13.5 & $(-8,6,-6)$ & 11.2 \\
\hline$(8,4,2)$ & 135.8 & $(-8,-4,-2)$ & 11.4 & $(7,-7,6)$ & 92.3 & $(-7,7,-6)$ & 7.6 \\
\hline$(1,-3,2)$ & 29.2 & $(-1,3,-2)$ & 2.5 & $(6,-8,6)$ & 62.0 & $(-6,8,-6)$ & 5.7 \\
\hline$(0,-4,2)$ & 927.8 & $(0,4,-2)$ & 86.9 & $(9,-8,7)$ & 78.4 & $(-9,8,-7)$ & 4.9 \\
\hline$(-1,-5,2)$ & 366.7 & $(1,5,-2)$ & 38.3 & $(8,-9,7)$ & 63.8 & $(-8,9,-7)$ & 3.5 \\
\hline$(-3,-7,2)$ & 140.6 & $(3,7,-2)$ & 10.3 & & & & \\
\hline
\end{tabular}


Table 3.4. The permitted reflections of la $\overline{3} d$ and observed reflections for IX35-30. Listed h, k, 1, are permilted reflection for Cubic Aspects. + indicales presence of reflection. - indicates absence of reflection. $s=h^{2}+k^{2}+l^{2}$. Reflections with $s \leq 100$ are listed. Table from reference ${ }^{104}$.

\begin{tabular}{|c|c|c|c|c|c|c|c|c|c|c|c|}
\hline s & h & k & l & la $3 l$ & observed & s & h & k & l & Ia $3 l$ & observed \\
\hline l & 1 & 0 & 0 & - & - & 30 & 5 & 2 & l & + & + \\
\hline 2 & 1 & 1 & 0 & - & - & 32 & 4 & 4 & 0 & + & + \\
\hline 3 & 1 & 1 & 1 & - & - & 33 & 5 & 2 & 2 & - & - \\
\hline 4 & 2 & 0 & 0 & - & - & 33 & 4 & 4 & 1 & - & - \\
\hline 5 & 2 & 1 & 0 & - & - & 34 & 5 & 3 & 0 & - & - \\
\hline 6 & 2 & 1 & l & + & + & 34 & 4 & 3 & 3 & - & - \\
\hline 8 & 2 & 2 & 0 & + & + & 35 & 5 & 3 & 1 & - & - \\
\hline 9 & 3 & 0 & 0 & - & - & 36 & 6 & 0 & 0 & - & - \\
\hline 9 & 2 & 2 & 1 & - & - & 36 & 4 & 4 & 2 & - & - \\
\hline 10 & 3 & 1 & 0 & - & - & 37 & 6 & 1 & 0 & - & - \\
\hline 11 & 3 & 1 & 1 & - & - & 38 & 6 & 1 & 1 & + & + \\
\hline 12 & 2 & 2 & 2 & - & - & 38 & 5 & 3 & 2 & + & + \\
\hline 13 & 3 & 2 & 0 & - & - & 40 & 6 & 2 & 0 & + & + \\
\hline 14 & 3 & 2 & 1 & + & + & 41 & 6 & 2 & 1 & - & - \\
\hline 16 & 4 & 0 & 0 & + & - & 41 & 5 & 4 & 0 & - & - \\
\hline 17 & 4 & 1 & 0 & - & - & 41 & 4 & 4 & 3 & - & - \\
\hline 17 & 3 & 2 & 2 & - & - & 42 & 5 & 4 & 1 & + & + \\
\hline 18 & 4 & 1 & 1 & - & - & 43 & 5 & 3 & 3 & - & - \\
\hline 18 & 3 & 3 & 0 & - & - & 44 & 6 & 2 & 2 & - & - \\
\hline 19 & 3 & 3 & 1 & - & - & 45 & 6 & 3 & 0 & - & - \\
\hline 20 & 4 & 2 & 0 & + & + & 45 & 5 & 4 & 2 & - & - \\
\hline 21 & 4 & 2 & 1 & - & - & 46 & 6 & 3 & 1 & + & + \\
\hline 22 & 3 & 3 & 2 & + & + & 48 & 4 & 4 & 4 & + & + \\
\hline 24 & 4 & 2 & 2 & + & + & 49 & 7 & 0 & 0 & - & - \\
\hline 25 & 5 & 0 & 0 & - & - & 49 & 6 & 3 & 2 & - & - \\
\hline 25 & 4 & 3 & 0 & - & - & 50 & 7 & 1 & 0 & - & - \\
\hline 26 & 5 & 1 & 0 & - & - & 50 & 5 & 5 & 0 & - & - \\
\hline 26 & 4 & 3 & 1 & + & + & 50 & 5 & 4 & 3 & + & + \\
\hline 27 & 5 & 1 & 1 & - & - & 51 & 7 & 1 & 1 & - & - \\
\hline 27 & 3 & 3 & 3 & - & - & 51 & 5 & 5 & 1 & - & - \\
\hline 29 & 5 & 2 & 0 & - & - & 52 & 6 & 4 & 0 & + & + \\
\hline 29 & 4 & 3 & 2 & - & - & 53 & 7 & 2 & 0 & - & - \\
\hline & & & & & & & & & \\
\hline
\end{tabular}




\begin{tabular}{|c|c|c|c|c|c|c|c|c|c|c|c|}
\hline $\mathrm{s}$ & $\mathrm{h}$ & $\mathrm{k}$ & $\mathrm{l}$ & Ia 3 d & observed & $\mathrm{s}$ & $\mathrm{h}$ & $\mathrm{k}$ & $\mathrm{l}$ & Ia $\overline{3} d$ & observed \\
\hline 53 & 6 & 4 & 1 & - & - & 78 & 7 & 5 & 2 & + & - \\
\hline 54 & 7 & 2 & 1 & + & - & 80 & 8 & 4 & 0 & + & + \\
\hline 54 & 6 & 3 & 3 & + & + & 81 & 9 & 0 & 0 & - & - \\
\hline 54 & 5 & 5 & 2 & + & - & 81 & 8 & 4 & 1 & - & - \\
\hline 56 & 6 & 4 & 2 & + & + & 81 & 7 & 4 & 4 & - & - \\
\hline 57 & 7 & 2 & 2 & - & - & 81 & 6 & 6 & 3 & - & - \\
\hline 57 & 5 & 4 & 4 & - & - & 82 & 9 & 1 & 0 & - & - \\
\hline 58 & 7 & 3 & 0 & - & - & 82 & 8 & 3 & 3 & - & - \\
\hline 59 & 7 & 3 & 1 & - & - & 83 & 9 & 1 & 1 & - & - \\
\hline 59 & 5 & 5 & 3 & - & - & 83 & 7 & 5 & 3 & - & - \\
\hline 61 & 6 & 5 & 0 & - & - & 84 & 8 & 4 & 2 & + & + \\
\hline 61 & 6 & 4 & 3 & - & - & 85 & 9 & 2 & 0 & - & - \\
\hline 62 & 7 & 3 & 2 & + & + & 85 & 7 & 6 & 0 & - & - \\
\hline 62 & 6 & 5 & 1 & + & + & 86 & 9 & 2 & 1 & + & - \\
\hline 64 & 8 & 0 & 0 & + & - & 86 & 7 & 6 & 1 & + & - \\
\hline 65 & 8 & 1 & 0 & - & - & 86 & 6 & 5 & 5 & + & + \\
\hline 65 & 7 & 4 & 0 & - & - & 88 & 6 & 6 & 4 & + & + \\
\hline 65 & 6 & 5 & 2 & - & - & 89 & 9 & 2 & 2 & - & - \\
\hline 66 & 8 & 1 & 1 & - & - & 89 & 8 & 5 & 0 & - & - \\
\hline 66 & 7 & 4 & 1 & + & - & 89 & 8 & 4 & 3 & - & - \\
\hline 66 & 5 & 5 & 4 & - & - & 89 & 7 & 6 & 2 & - & - \\
\hline 67 & 7 & 3 & 3 & - & - & 90 & 9 & 3 & 0 & - & - \\
\hline 68 & 8 & 2 & 0 & + & + & 90 & 8 & 5 & 1 & + & + \\
\hline 68 & 6 & 4 & 4 & - & - & 90 & 7 & 5 & 4 & + & + \\
\hline 69 & 8 & 2 & 1 & - & - & 91 & 9 & 3 & 1 & - & - \\
\hline 69 & 7 & 4 & 2 & - & - & 93 & 8 & 5 & 2 & - & - \\
\hline 70 & 6 & 5 & 3 & + & + & 94 & 9 & 3 & 2 & + & + \\
\hline 72 & 8 & 2 & 2 & + & + & 94 & 7 & 6 & 3 & + & - \\
\hline 72 & 6 & 6 & 0 & + & + & 96 & 8 & 4 & 4 & + & - \\
\hline 73 & 8 & 3 & 0 & - & - & 97 & 9 & 4 & 0 & - & - \\
\hline 73 & 6 & 6 & 1 & - & - & 97 & 6 & 6 & 5 & - & - \\
\hline 74 & 8 & 3 & 1 & + & - & 98 & 9 & 4 & 1 & + & + \\
\hline 74 & 7 & 5 & 0 & - & - & 98 & 8 & 5 & 3 & + & + \\
\hline 74 & 7 & 4 & 3 & + & + & 98 & 7 & 7 & 0 & - & - \\
\hline 75 & 7 & 5 & 1 & - & - & 99 & 9 & 3 & 3 & - & - \\
\hline 75 & 5 & 5 & 5 & - & - & 99 & 7 & 7 & 1 & - & - \\
\hline 76 & 6 & 6 & 2 & - & - & 99 & 7 & 5 & 5 & - & - \\
\hline 77 & 8 & 3 & 2 & - & - & 100 & 10 & 0 & 0 & - & - \\
\hline 77 & 6 & 5 & 4 & - & - & 100 & 8 & 6 & 0 & + & + \\
\hline
\end{tabular}




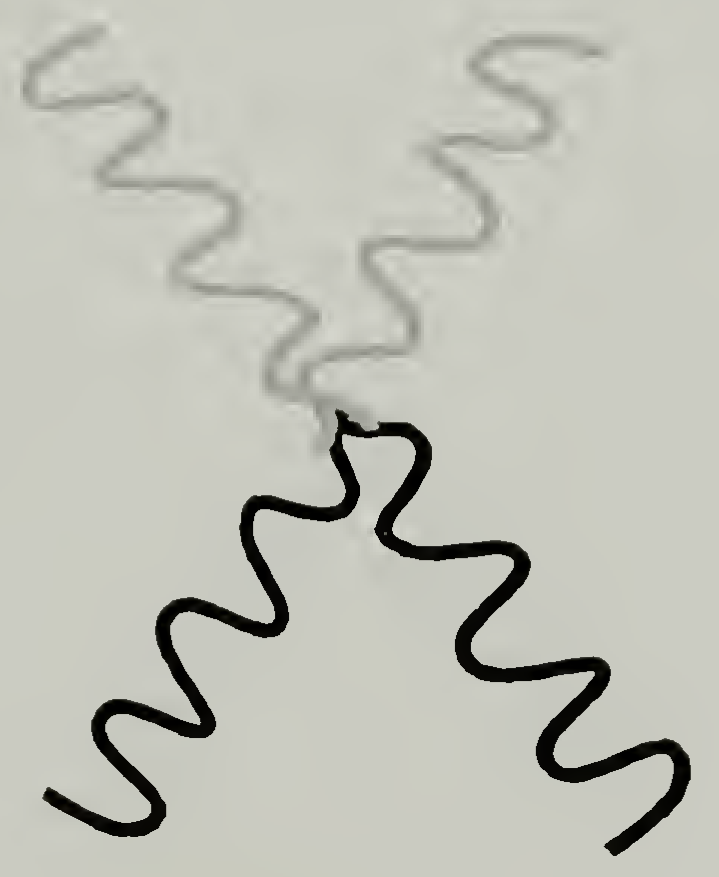

Figure 3.1. Illustrations of $\mathrm{A}_{2} \mathrm{~B}_{2}$ miktoarm star block copolymer architecture. 


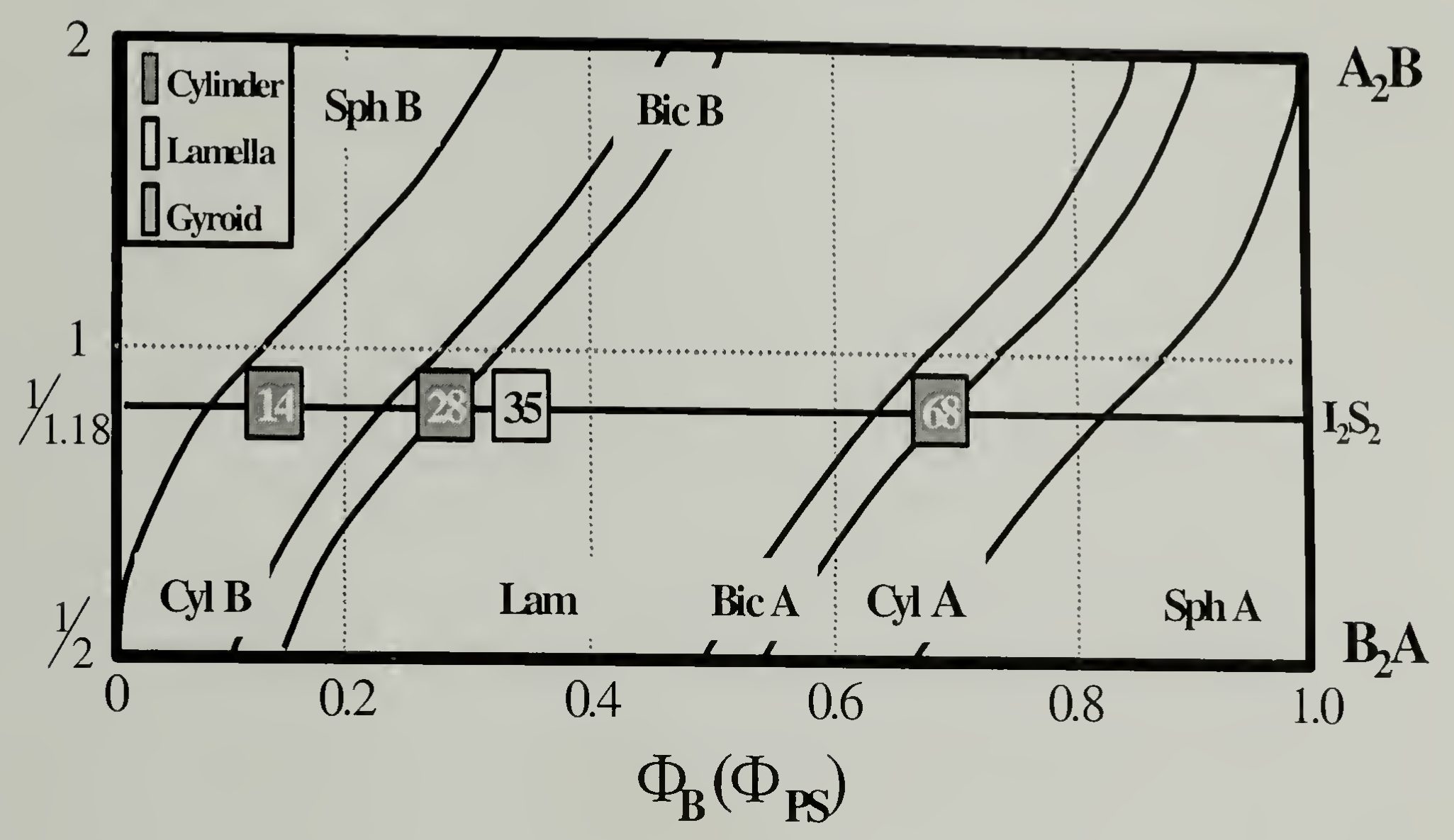

Figure 3.2. Morphology diagram with $\mathrm{A}_{2} \mathrm{~B}_{2}$ block copolymer sample positions indicated. The numbers in the symbols correspond to the sample numbers given in Table 3.1. 


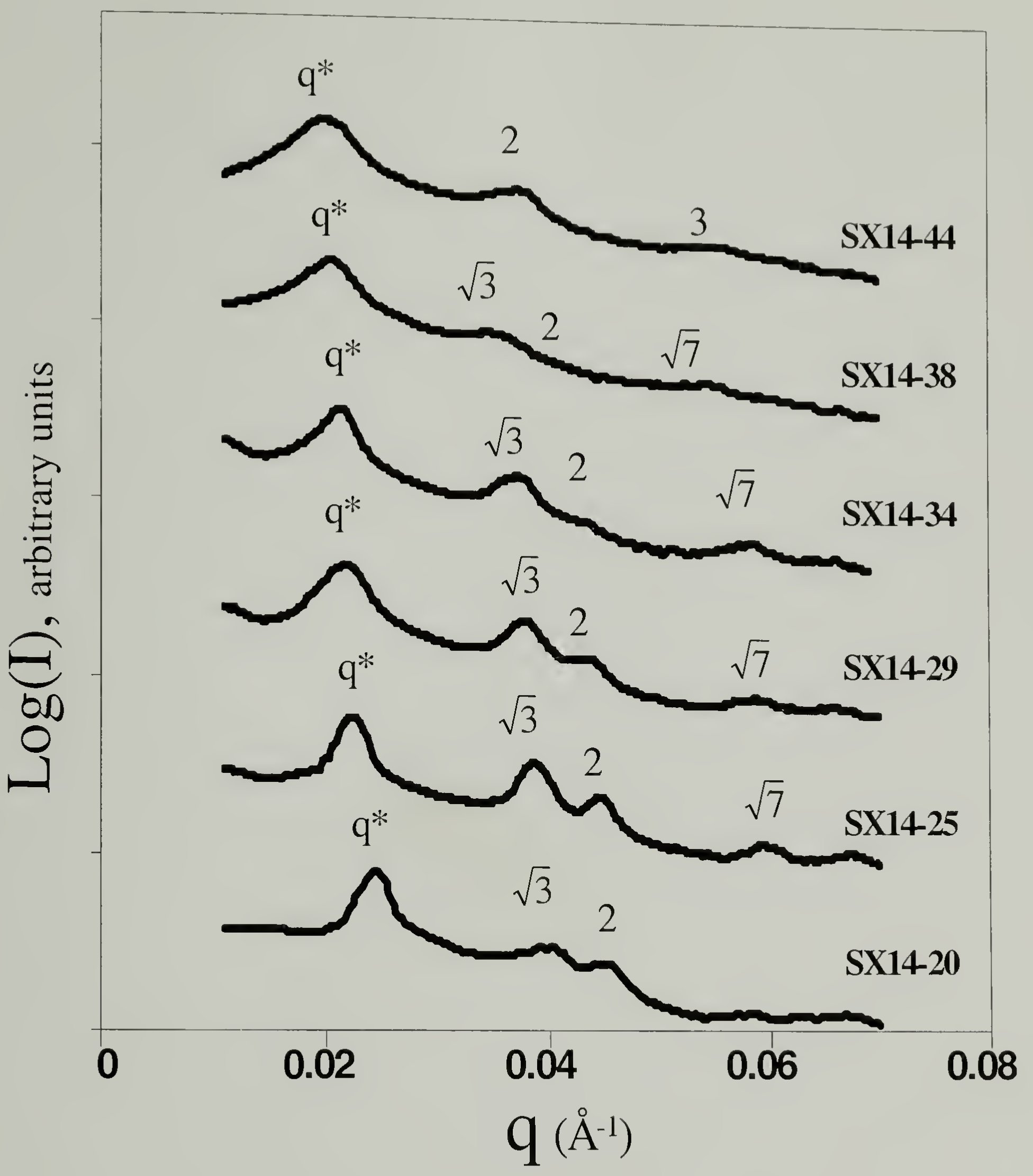

Figure 3.3. $\log (\mathrm{I})$ vs. q SAXS patterns for the SX14 blend series. The ratios $\mathrm{q}_{\mathrm{n}} / \mathrm{q}^{*}$ of the scattering vector of each peak to the scattering vector of the primary peak are indicated. 


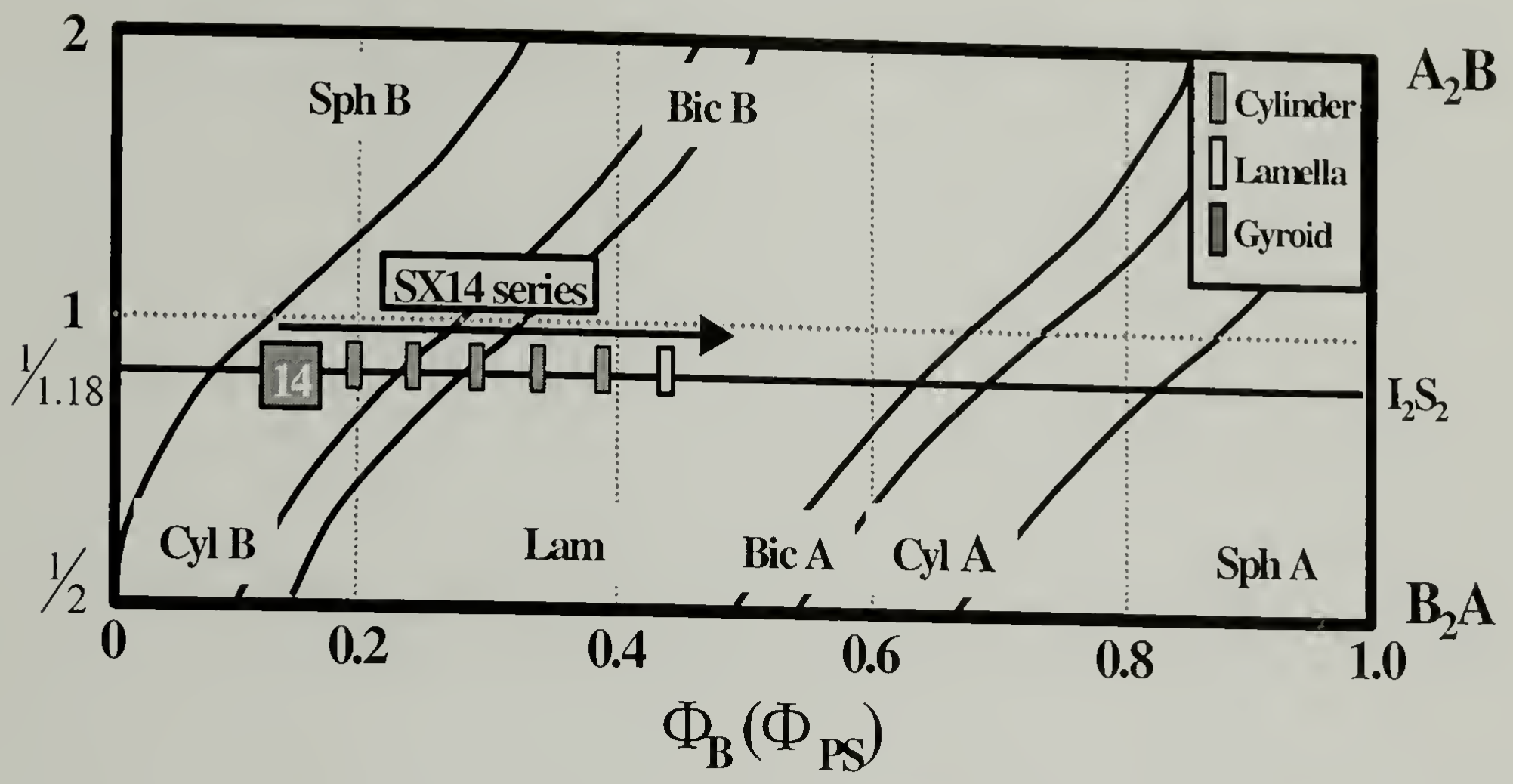

Figure 3.4. Blend series SX14 mapped onto the theoretical morphology diagram. The numbered symbols along the solid line at $\varepsilon=1 / 1.18$ indicate the volume fractions of the pure $I_{2} S_{2}$ samples upon which the blends are based. The locations on the diagrams of small rectangular boxes indicate the volume fractions of the blends in the series. The shading of the boxes indicates the morphology. 


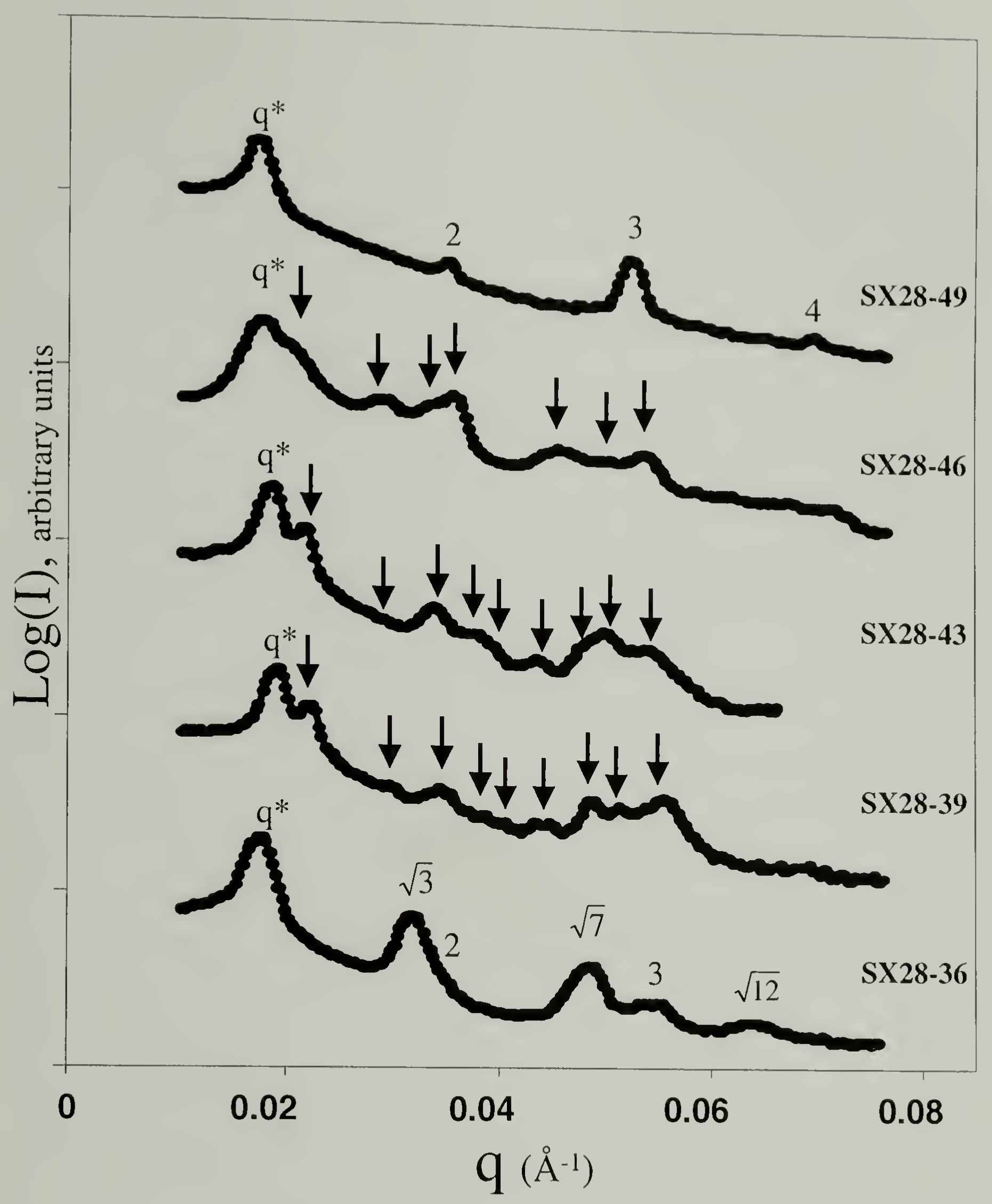

Figure 3.5. $\log (\mathrm{I})$ vs. q SAXS patterns for the SX14 blend series. The ratios $\mathrm{q}_{\mathrm{n}} / \mathrm{q}^{*}$ of the scattering vector of each peak to the scattering vector of the primary peak are indicated. 


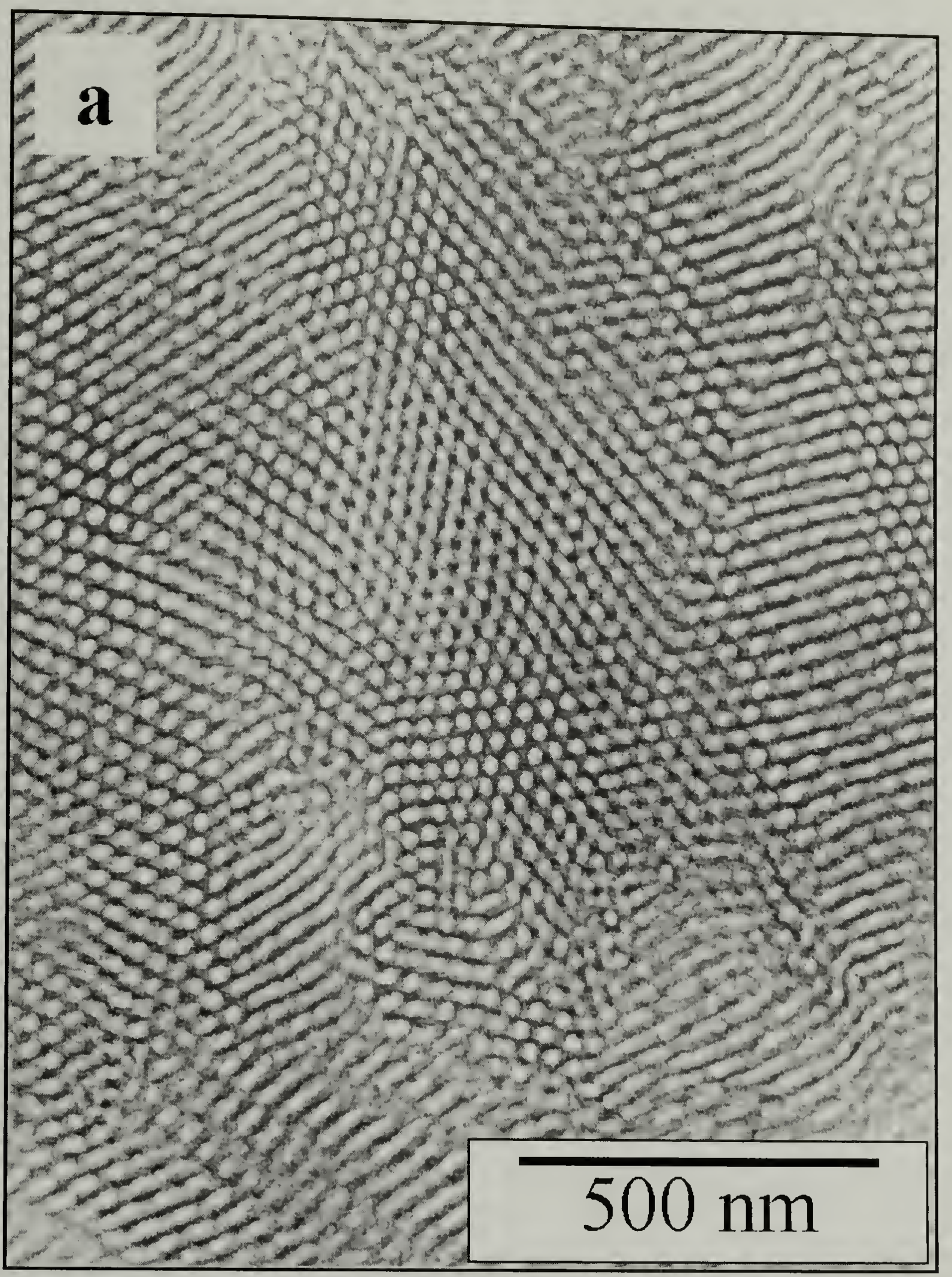




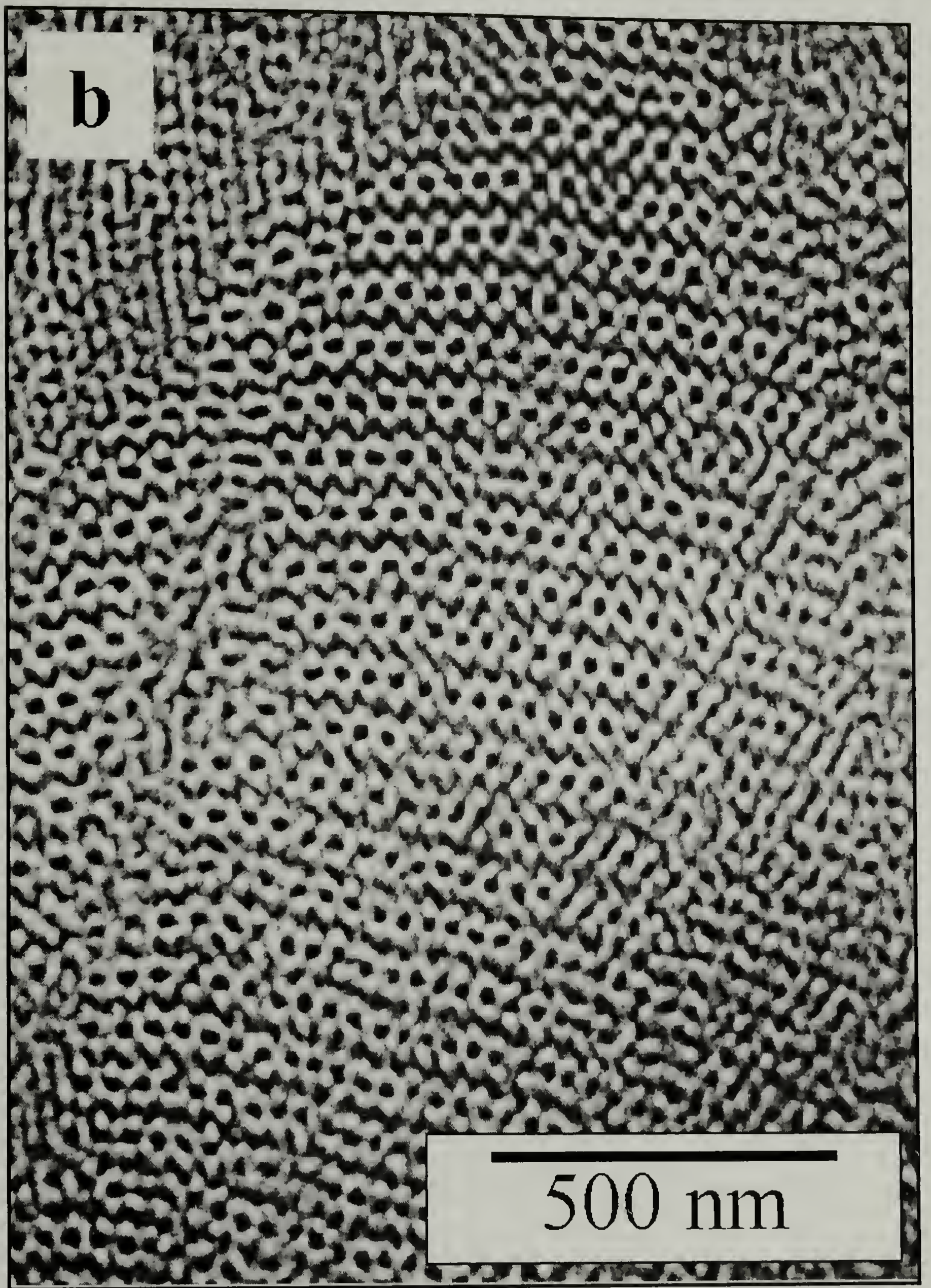




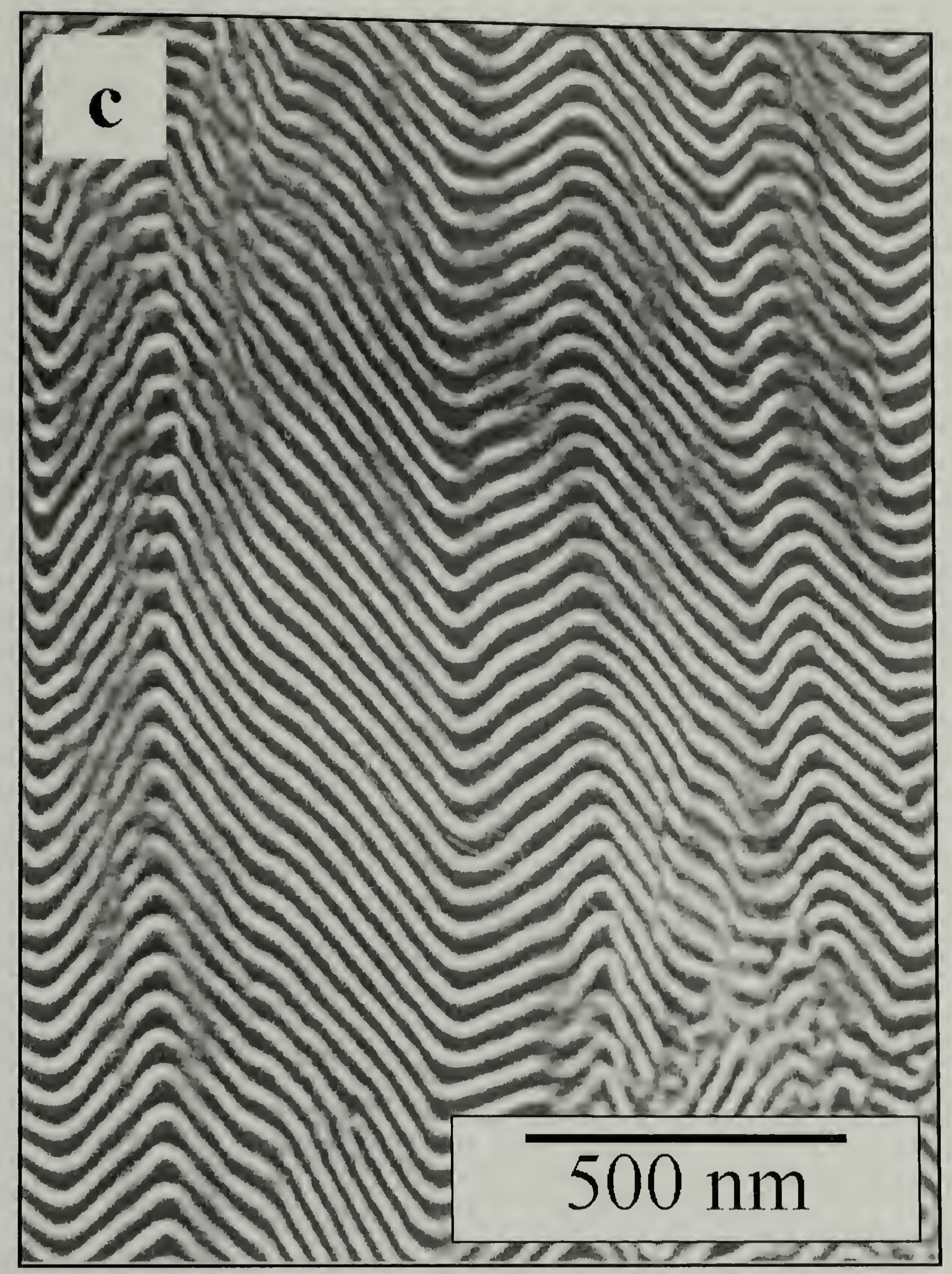

Figure 3.6. TEM of blend series SX28. a) SX28-36; b) SX28-46; c) SX28-49. 


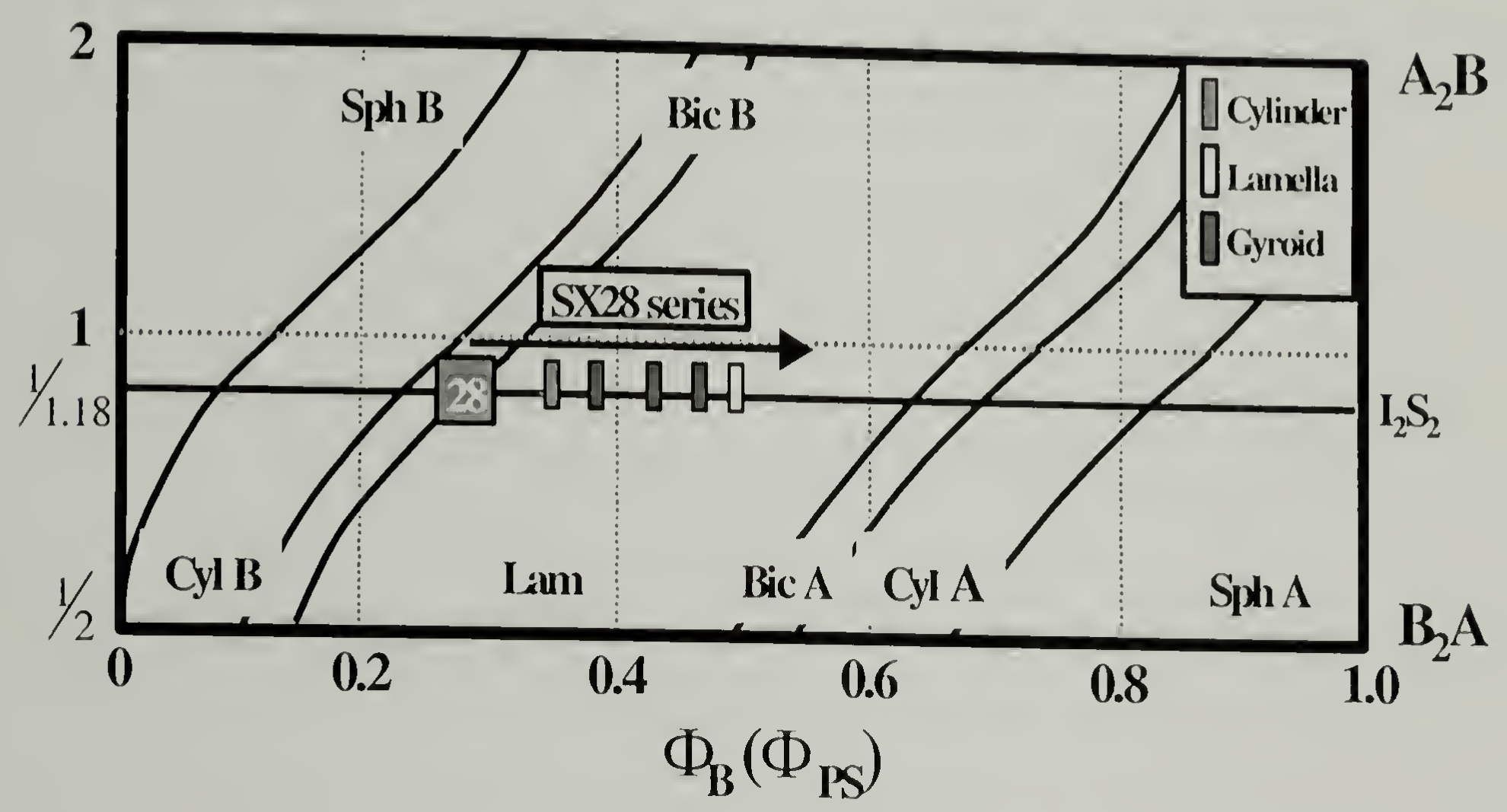

Figure 3.7. Blend series SX28 mapped onto the theoretical morphology diagram. The numbered symbols along the solid line at $\varepsilon=1 / 1.18$ indicate the volume fractions of the pure $\mathrm{I}_{2} \mathrm{~S}_{2}$ samples upon which the blends are based. The locations on the diagrams of small rectangular boxes indicate the volume fractions of the blends in the series. The shading of the boxes indicates the morphology. 


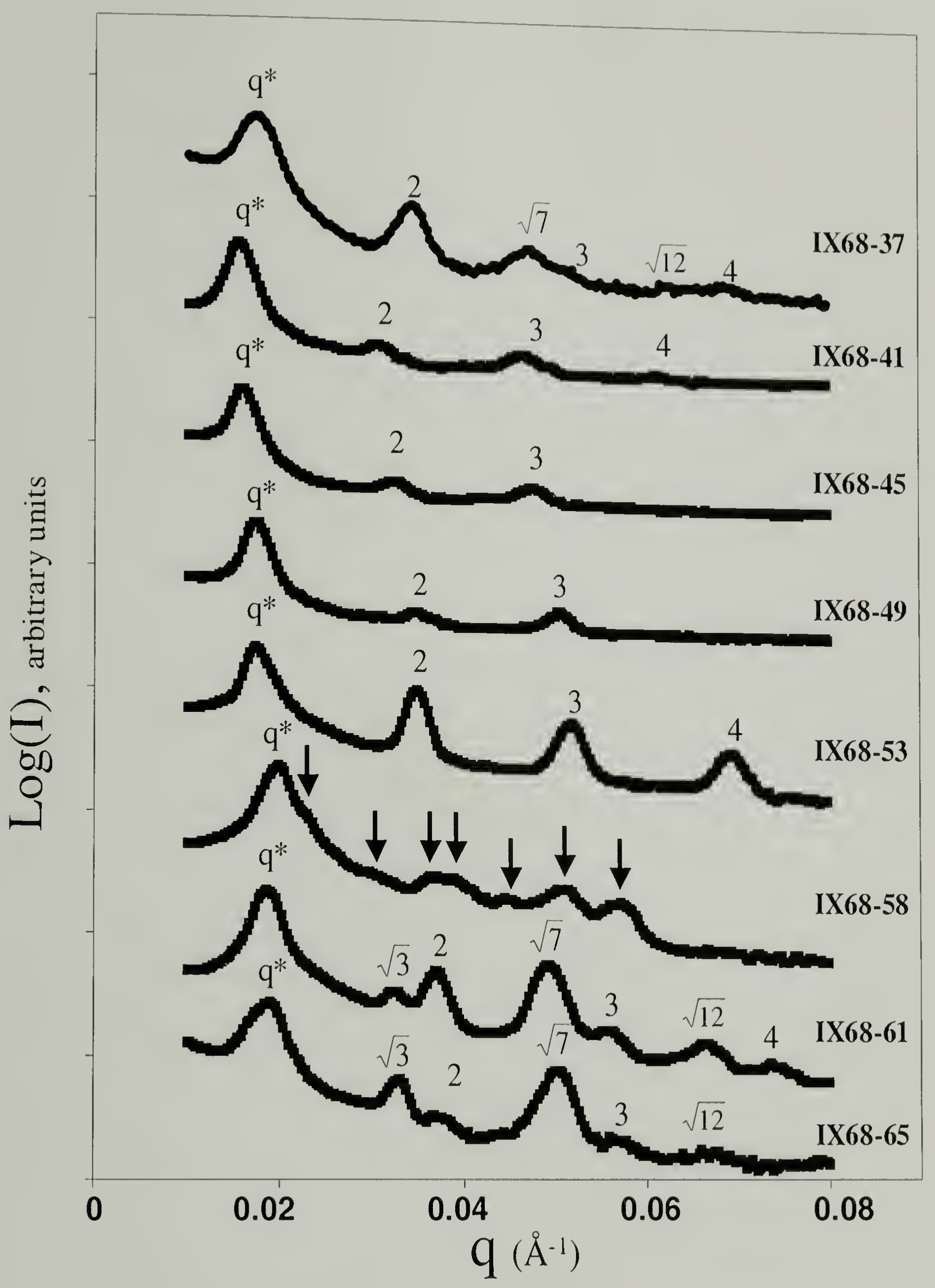

Figure 3.8. $\log (\mathrm{I})$ vs. q SAXS patterns for the IX68 blend series. The ratios $\mathrm{q}_{\mathrm{n}} / \mathrm{q}^{*}$ of the scattering vector of each peak to the scattering vector of the primary peak are indicated. 


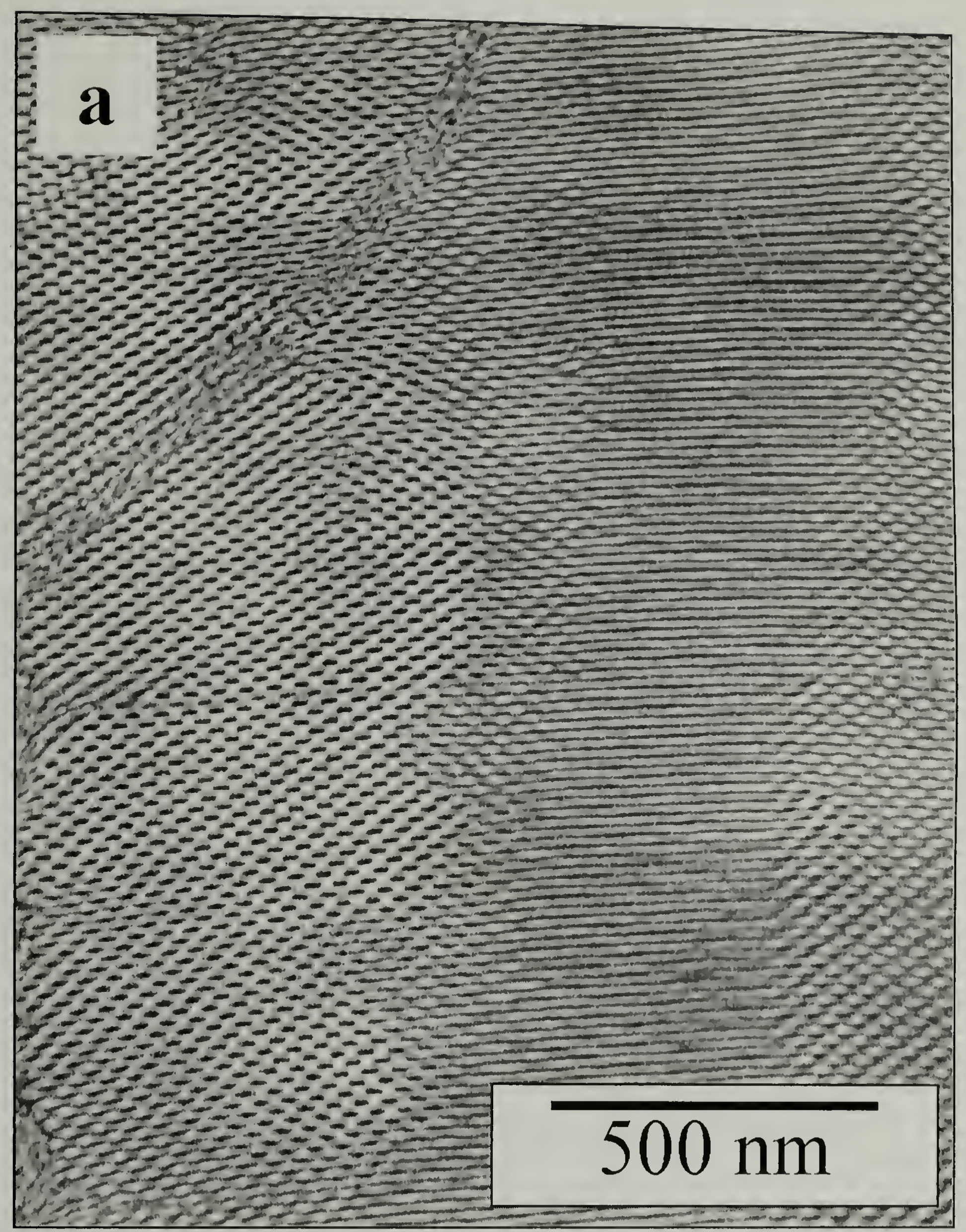




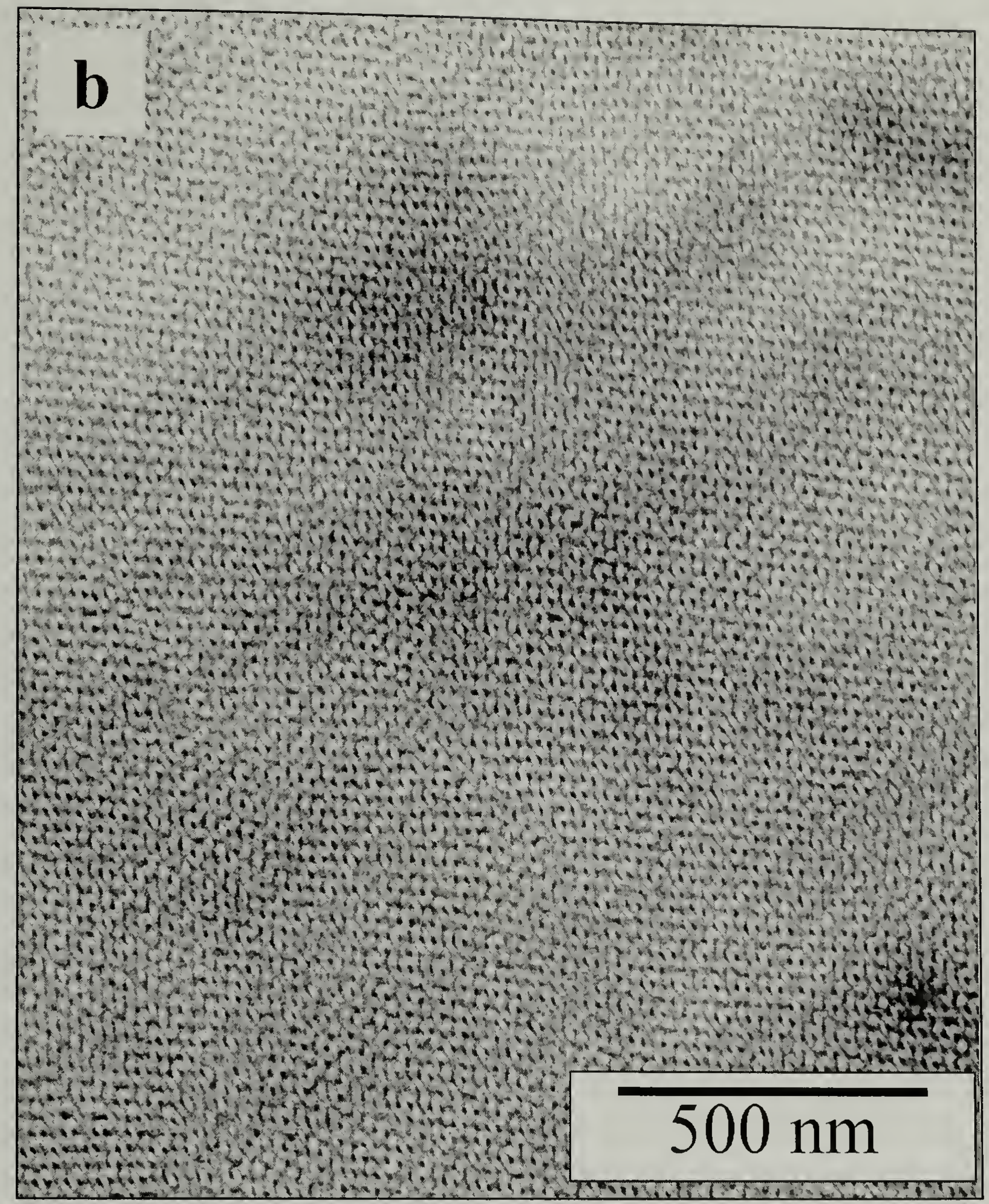




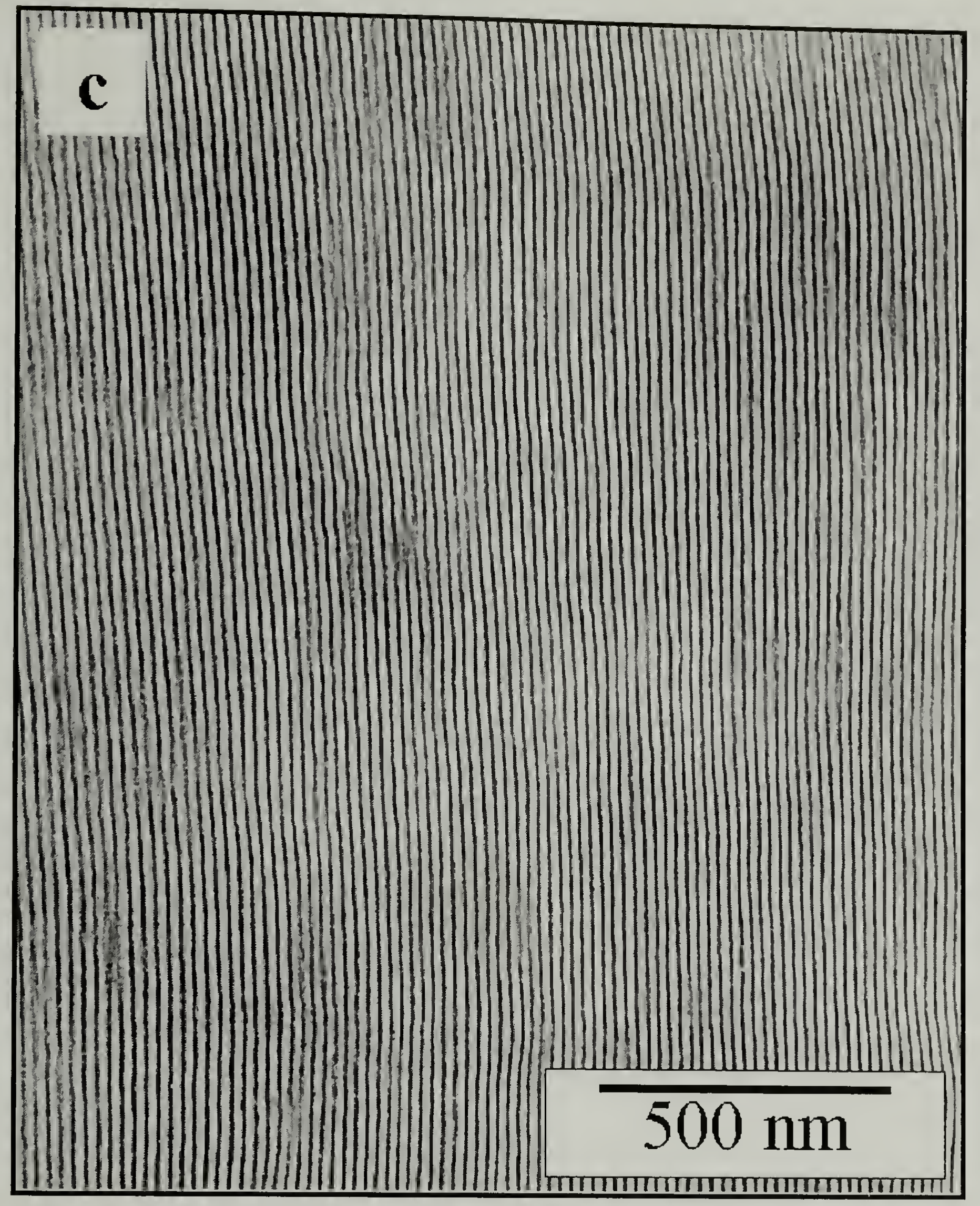




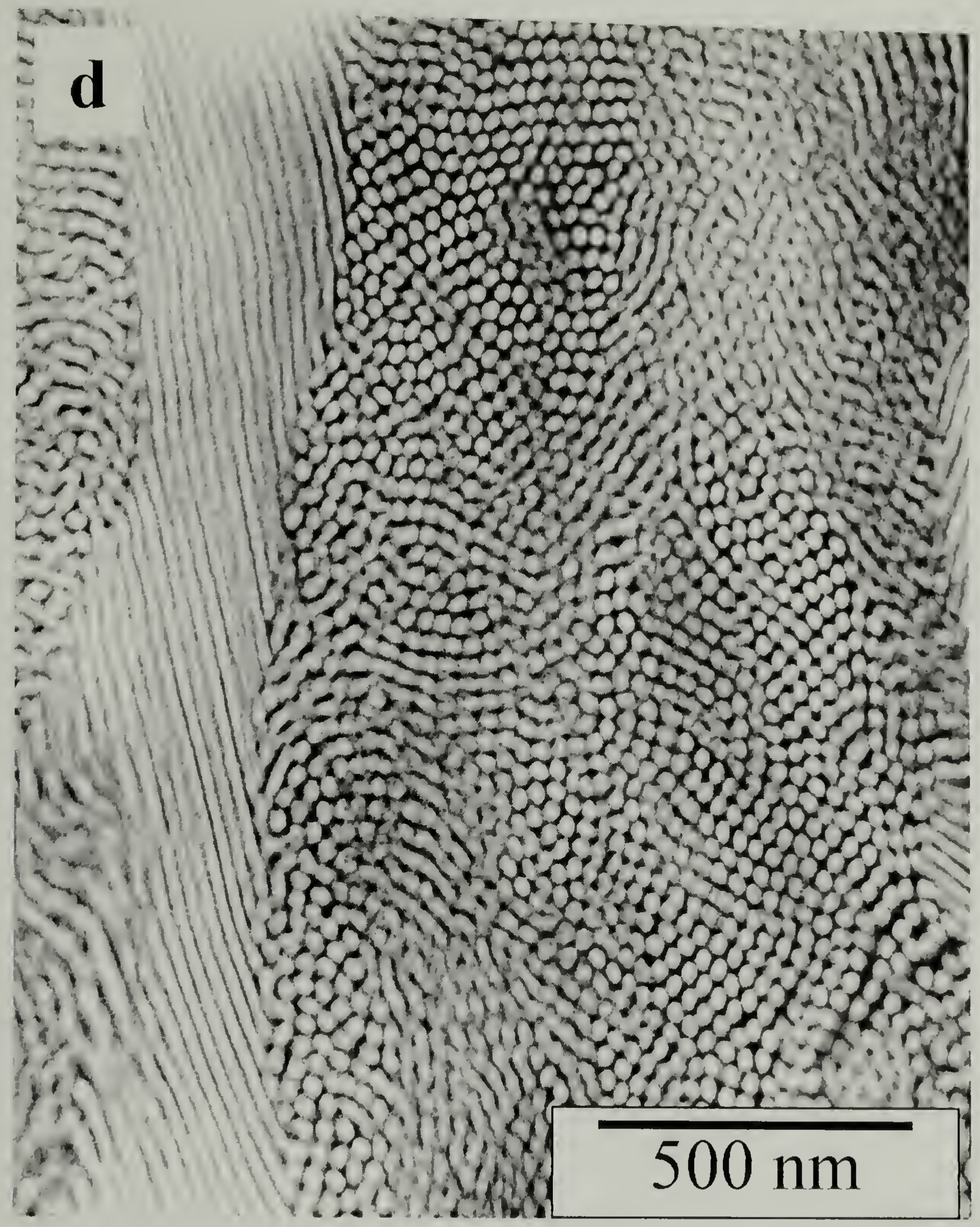

Figure 3.9. TEM of IX68 blend series. a) IX68-65; b) IX68-58; c) IX68-45; d) IX68-37. 


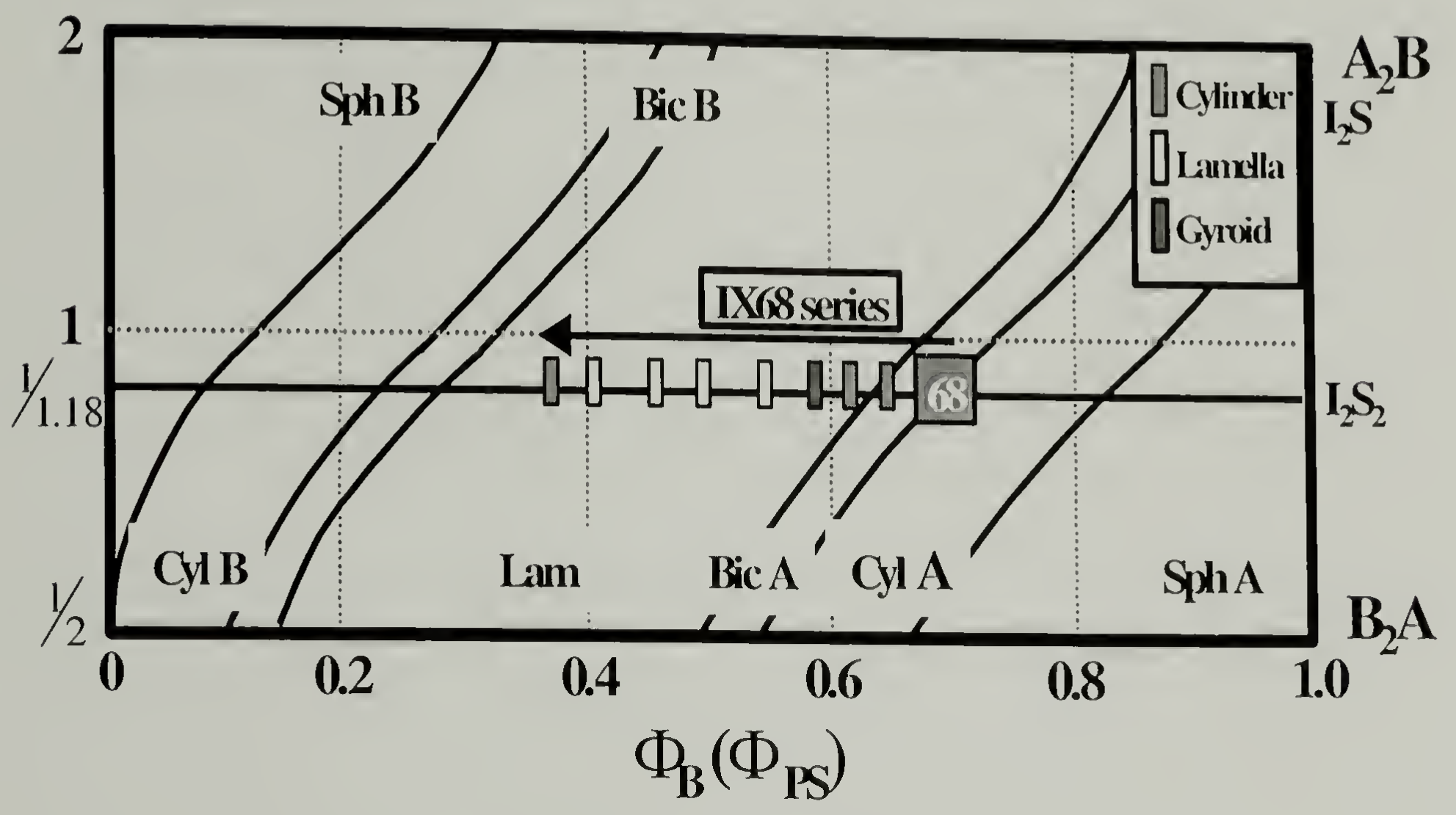

Figure 3.10. Blend series IX68 mapped onto the theoretical morphology diagram. 


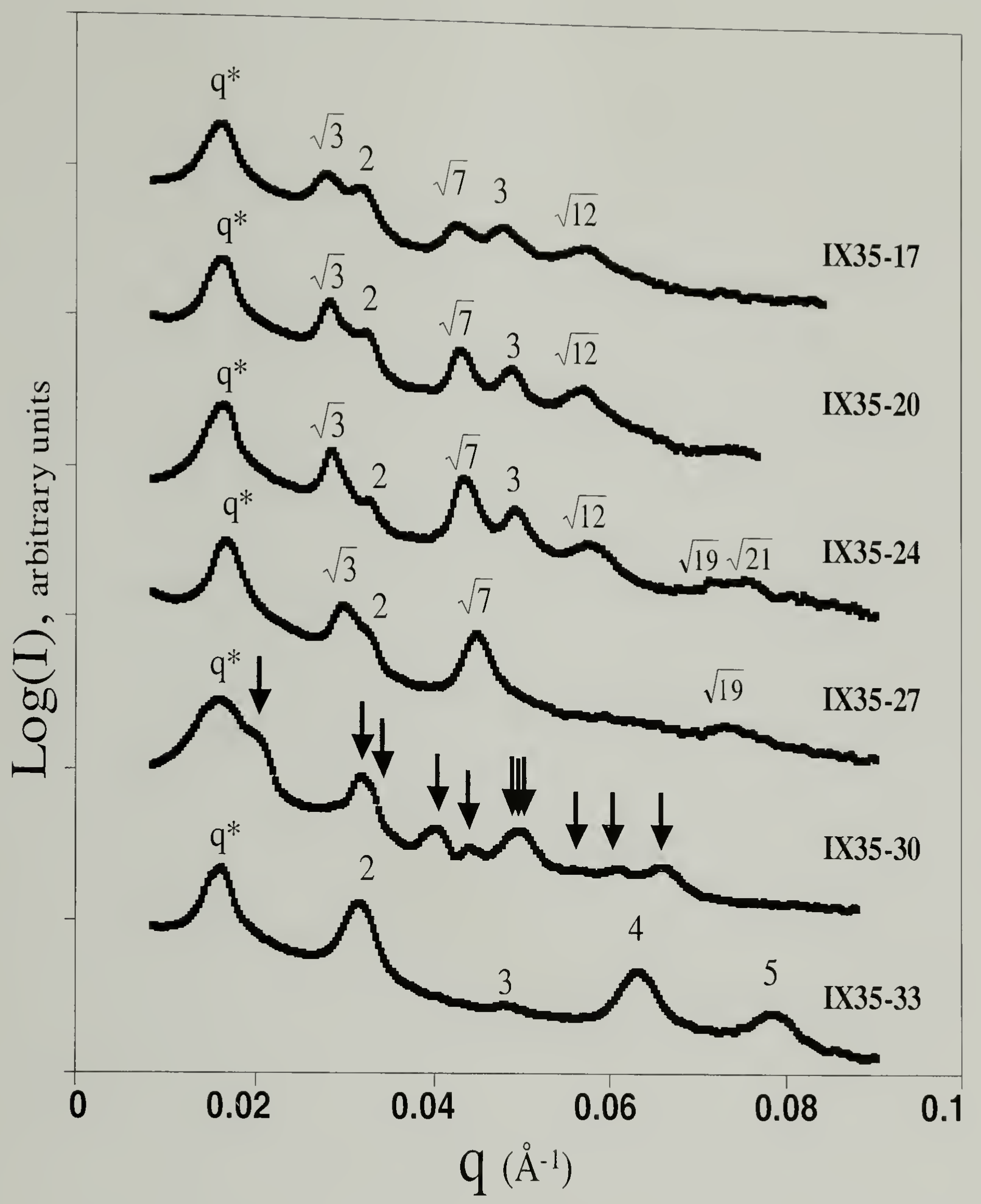

Figure 3.11. $\log (\mathrm{I})$ vs. q SAXS patterns for the IX68 blend series. The ratios $\mathrm{q}_{\mathrm{n}} / \mathrm{q}^{*}$ of the scattering vector of each peak to the scattering vector of the primary peak are indicated. 
a
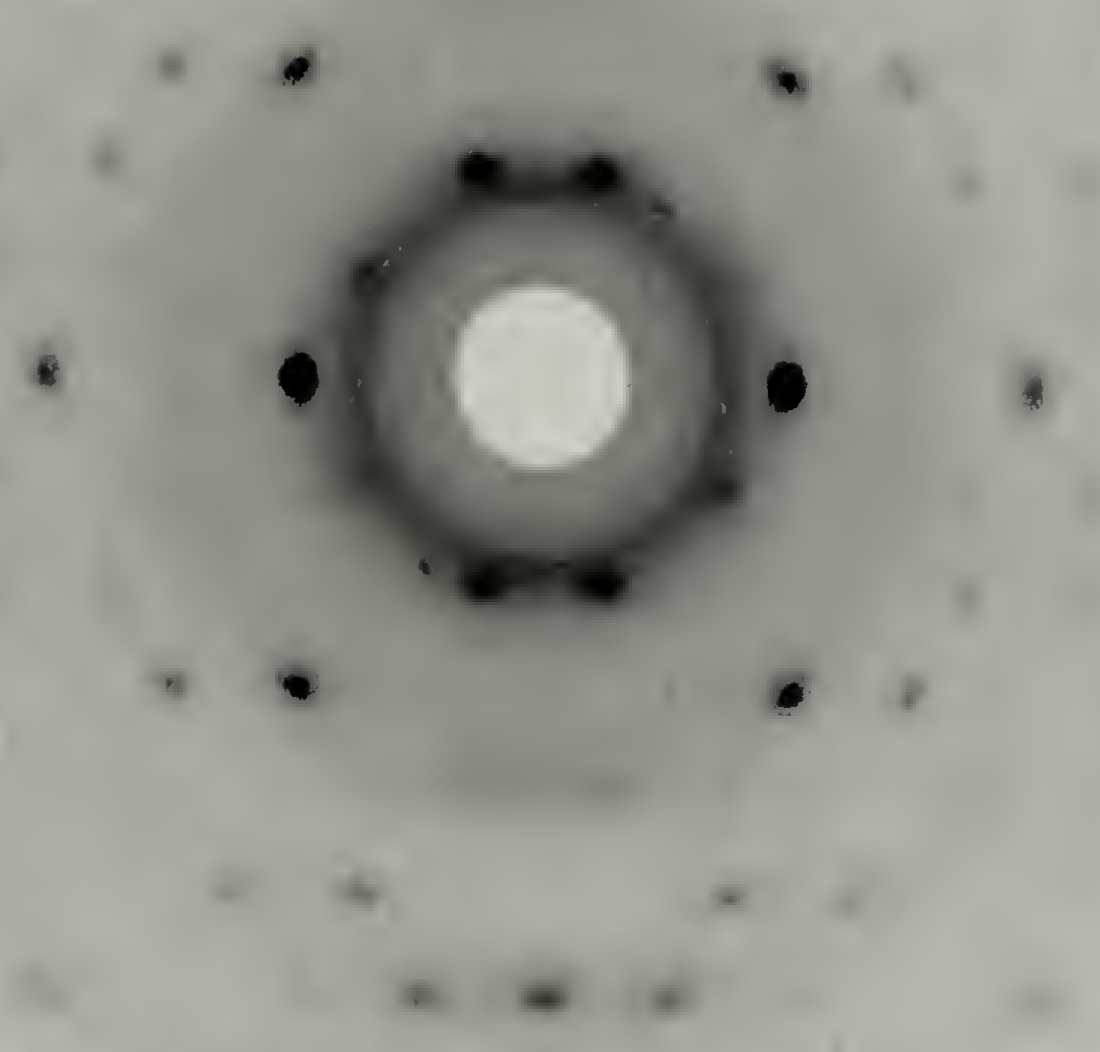
b

$897 \quad 987$
$-\quad 0$

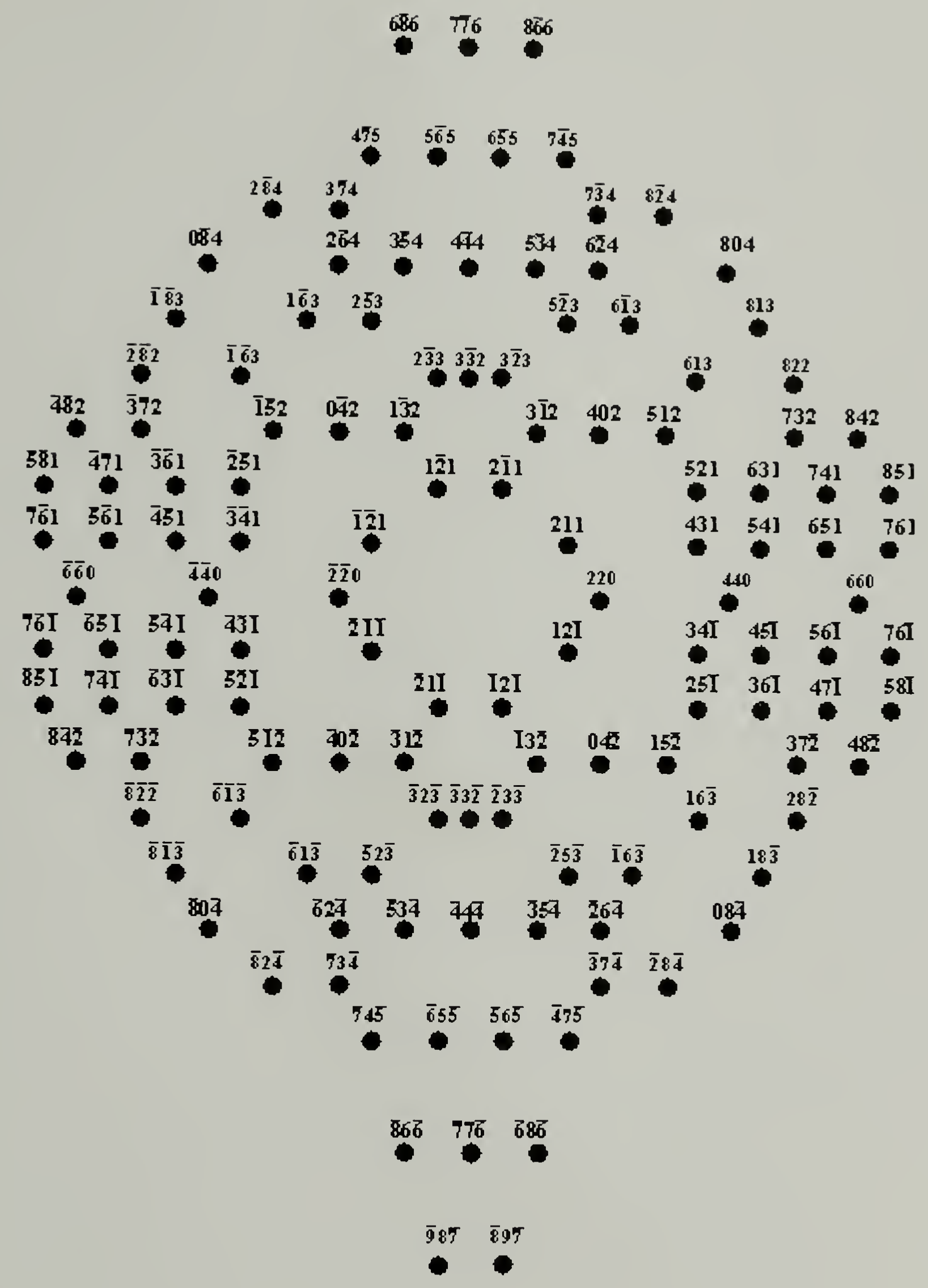

Figure 3.12. Two dimensional single crystal SAXS pattern of IX35-30 gyroid structure (a) pattern (b) indexed schematic. 


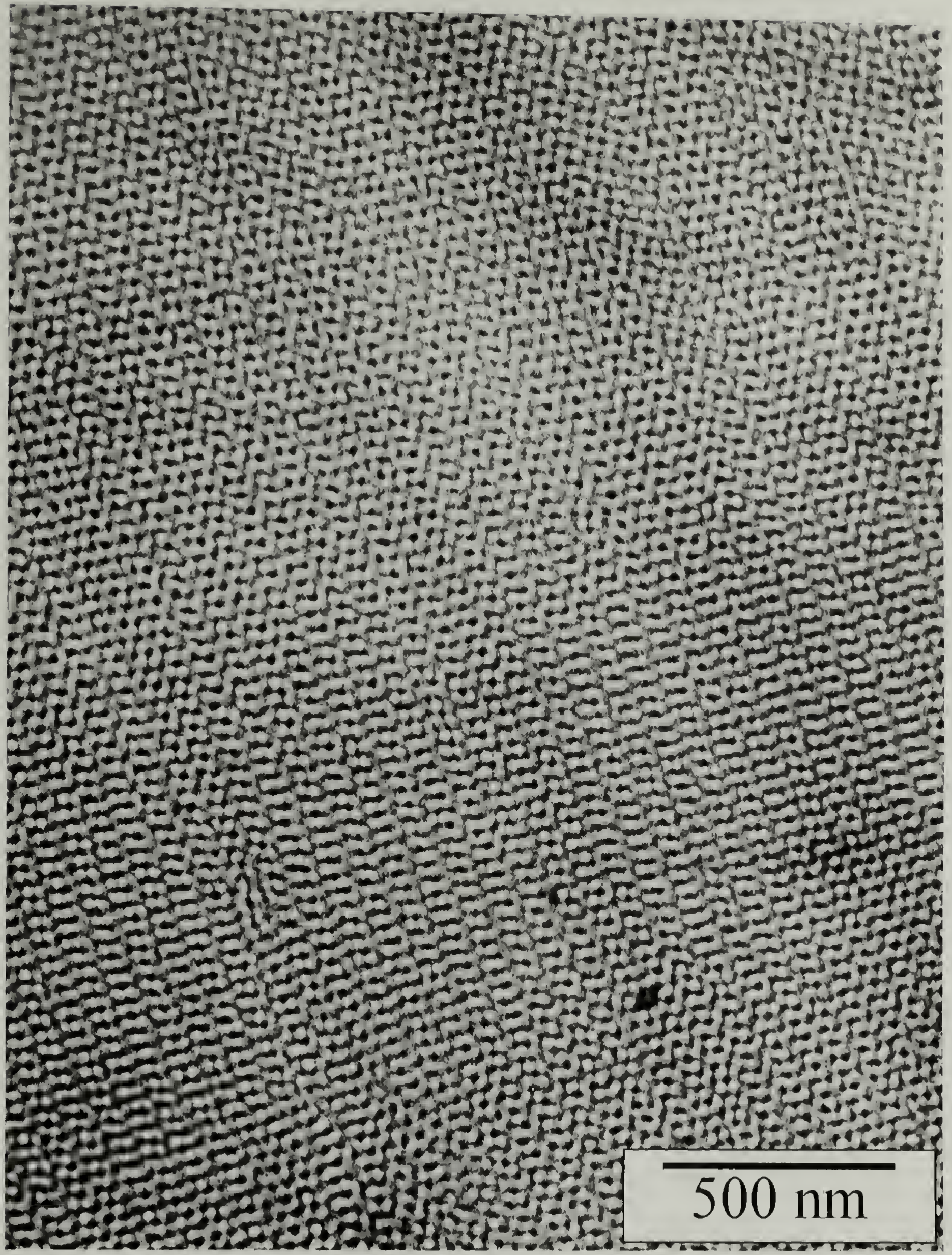

Figure 3.13. TEM of IX35-32. 


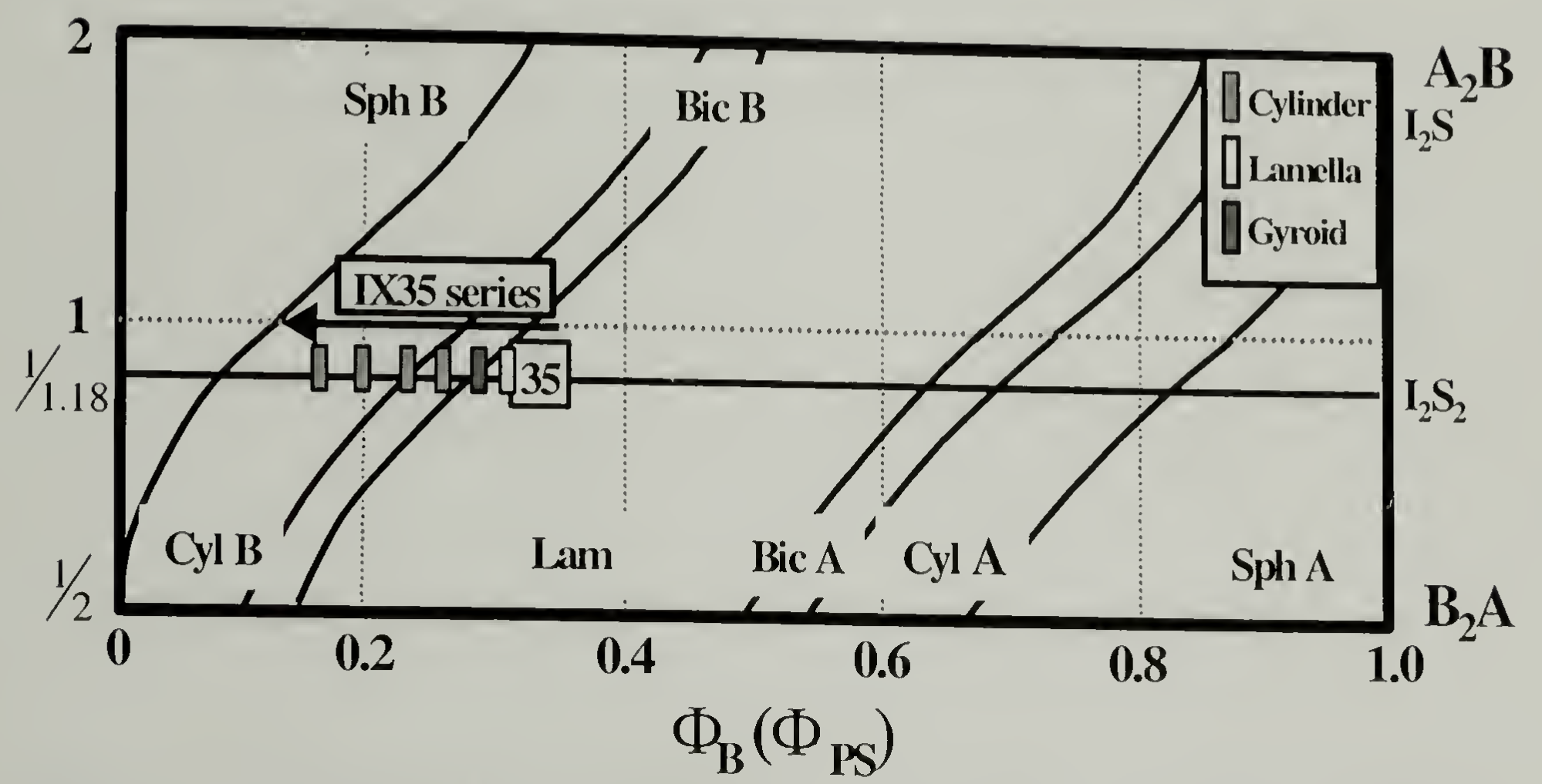

Figure 3.14. Blend series IX35 mapped onto the theoretical morphology diagram. 


\section{CHAPTER 4}

\section{MORPHOLOGICAL BEHAVIOR I 5 S MIKTOARM STAR BLOCK \\ COPOLYMERS:PACKING CONSTRAINTS ON MORPHOLOGY AND \\ DISCONTINUOUS CHEVRON TILT GRAIN BOUNDARIES}

\subsection{Abstract}

A morphological study of three $I_{5} S$ six-arm miktoarm star block copolymers and one $\mathrm{I}_{5}-\mathrm{S}-\mathrm{I}_{5}$ dumbbell shaped graft block copolymer is presented. The miktoarm stars are comprised of five arms of polyisoprene (PI) and one arm of polystyrene (PS) joined together at a single junction point; the dumbbell shaped graft block copolymer is comprised of five arms of PI on each end of a single connecting block of PS. The strong segregation limit theory for the morphological behavior of miktoarm stars, predicts that these materials should form spherical morphologies, but only lamellar and cylindrical morphologies were observed by TEM and SAXS. These results are similar to previously reported discrepancies between experimentally observed morphological behaviors of miktoarm stars and the predictions of the theory. Previous work has attributed the discrepancies to the neglect of the effect of the multifunctional junction points on calculated free energies. The current results suggest that in addition to this, geometrical packing constraints prevent the formation of morphologies such as spheres and cylinders in highly asymmetric miktoarm stars in which the minor volume fraction component would need to occupy the matrix phase. Finally, unusual broken chevron tilt grain boundary morphologies were observed in a lamellar $\mathrm{I}_{5} \mathrm{~S}$ material. We attribute 
these new structures to the asymmetric energy penalties for interfacial bending which result from the molecular asymmetry of the miktoarm stars.

\subsection{Introduction}

Branched and grafted molecular architectures have been shown to be an additional factor (in addition to volume fraction and degree of segregation) which affects the morphological behavior of block copolymers. Guided by the predictions of a mean field theory derived by Milner ${ }^{11,83}$ for the morphological behavior of $A_{n} B_{m^{-}}$ type, miktoarm star block copolymers, work has focused on molecules with an array of architectures including $\mathrm{A}_{2} \mathrm{~B}$ stars, ${ }^{1,7,87-89} \mathrm{~A}_{3} \mathrm{~B}$ stars, ${ }^{90} \mathrm{~A}_{\mathrm{n}} \mathrm{B}_{\mathrm{n}}$ stars, ${ }^{9,10,105} \mathrm{~A}_{5} \mathrm{~B}$ stars ${ }^{8}$ and multigraft architectures, which may be considered to be linear combinations of miktoarm stars. ${ }^{3,106,107}$ These studies have borne out the predictions of the model in general, but have revealed systematic discrepancies. In the current study we further examine $\mathrm{I}_{5} \mathrm{~S}$ miktoarm star block copolymers, and an $\mathrm{I}_{5}-\mathrm{S}-\mathrm{I}_{5}$ material; the molecular architectures of these materials are illustrated in Figure 4.1. $I_{5} \mathrm{~S}$ stars have five arms of PI and one arm of PS per molecule, joined to each other at a single junction point. The dumbbell shaped $\mathrm{I}_{5}-\mathrm{S}-\mathrm{I}_{5}$ molecule is comprised of five arms of PI on each end of a connecting block of PS. These materials equal the highest architectural asymmetries investigated thus far. ${ }^{8}$

The morphology of $I_{5} S$ miktoarm stars is predicted by the Milner theory, which is strictly applicable only in the strong segregation limit. For architecturally and conformationally asymmetric block copolymer stars of type $A_{n} B_{m}$, the theory predicts 
morphology as a function of $\mathrm{B}$ component volume fraction, $\phi_{\mathrm{B}}$, and a molecular asymmetry parameter, $\varepsilon=\left(n_{\mathrm{A}} / n_{\mathrm{B}}\right)\left(l_{\mathrm{A}} / l_{\mathrm{B}}\right)^{1 / 2}$. Here, $n_{\mathrm{A}}$ and $n_{\mathrm{B}}$ are the numbers of arms of block materials $\mathrm{A}$ and $\mathrm{B}$, and $l_{\mathrm{i}}=\left(\mathrm{V}_{\mathrm{i}} / \mathrm{R}_{\mathrm{i}}{ }^{2}\right)=v_{\mathrm{i}} / \mathrm{b}_{\mathrm{i}}{ }^{2} . \mathrm{V}_{\mathrm{i}}$ and $\mathrm{R}_{\mathrm{i}}$ are the volume and radius of gyration of one arm of polymer $i$, while $v_{\mathrm{i}}$ is the segmental volume and $b_{\mathrm{i}}$ the statistical segment length of component $i$. Using segmental volumes of $132 \AA^{3}$ (PI) and $176 \AA^{3}$ (PS) and statistical segment lengths of $6.8 \AA$ (PI) and $6.9 \AA$ (PS), an $\varepsilon$ of 4.4 is calculated for these materials. ${ }^{108,109}$ The samples characterized have PS volume fractions of $0.58\left(\mathrm{I}_{5} \mathrm{~S}-58\right), 0.46\left(\mathrm{I}_{5} \mathrm{~S}-46\right), 0.37\left(\mathrm{I}_{5} \mathrm{~S}-37\right)$, and $0.66\left(\mathrm{I}_{5}-\mathrm{S}-\mathrm{I}_{5}-66\right)$.

\subsection{Experimental}

Three $I_{5} S$ miktoarm stars of PI and PS were synthesized using anionic polymerization and controlled chlorosilane chemistry. The synthesis of the $\mathrm{A}_{5} \mathrm{~B}$ and $\mathrm{A}_{5}-\mathrm{B}-\mathrm{A}_{5}$ type nonlinear block copolymers has been described in detail elsewhere. ${ }^{110}$ All manipulations were performed in glass reactors under high vacuum. The reactors were previously washed with benzene solution of $n$-butyllithium and rinsed with benzene. Benzene was the solvent for all polymerizations and linking reactions. The PS and PI arms were synthesized separately using sec-butyllithium as initiator and then linked together with the hexafunctional chlorosilane 1,2-bis-trichlorosilylethane $\left(\mathrm{Si}_{2} \mathrm{Cl}_{6}\right)$. The key synthetic step for $\mathrm{I}_{5} \mathrm{~S}$ was the preparation of PS-Si ${ }_{2} \mathrm{Cl}_{5}$, from PS-Li and $\mathrm{Si}_{2} \mathrm{Cl}_{6}$. For $\mathrm{I}_{5}-\mathrm{S}-\mathrm{I}_{5}$ the critical step is the preparation of $(\mathrm{PI})_{5}-\mathrm{Si}_{2} \mathrm{Cl}$, from PI-Li and $\mathrm{Si}_{2} \mathrm{Cl}_{6}$. The $\mathrm{I}_{5} \mathrm{~S}$ and $\mathrm{I}_{5}-\mathrm{S}-\mathrm{I}_{5}$ architectures were obtained by reaction of PI-Li with PS$\mathrm{Si}_{2} \mathrm{Cl}_{5}$, and of $\alpha, \omega$-dilithium PS with $(\mathrm{PI})_{5}-\mathrm{Si}_{2} \mathrm{Cl}$ respectively. 
Table 4.1 lists the molecular characteristics of each sample. Membrane osmometry (MO) was performed in toluene at $35^{\circ} \mathrm{C}$. Size-exclusion chromatography (SEC) with both refractive index and UV detectors $(\lambda=262 \mathrm{~nm})$ was performed in tetrahydrofuran at $30^{\circ} \mathrm{C}$ on individual PI and PS arms prior to silane coupling as well as on the overall miktoarm architectures. Vapor pressure osmometry (VPO) was performed in toluene at $50^{\circ} \mathrm{C}$. Proton nuclear magnetic resonance $\left({ }^{1} \mathrm{H}-\mathrm{NMR}\right)$ was used to determine the weight fraction of PS in each sample. Finally, the weight fraction of PS in each sample was calculated by dividing the PS $\operatorname{arm} M_{n}$, as measured by SEC, by the total $M_{n}$ of the star, $M_{n}(\mu)=5 \cdot\left(M_{n}(P I)\right)+M_{n}(P S)$. PS volume fraction was calculated for each sample using the mass fraction of PS measured by ${ }^{1} \mathrm{H}-\mathrm{NMR}$ and bulk densities. ${ }^{111}$

Bulk films were cast from 4 weight percent solutions in toluene, a nonselective solvent for PI and PS. ${ }^{112}$ Films approximately $2 \mathrm{~mm}$ thick were formed by allowing the solvent to evaporate slowly over a period of two weeks. The films were let stand at room temperature and atmospheric pressure for an additional week, then placed under vacuum at room temperature for one week to remove any residual solvent from the bulk material. The samples were subsequently annealed under vacuum for one week at $120^{\circ} \mathrm{C}$. The samples were then cooled under vacuum to room temperature over a period of several hours.

Sample morphology was characterized using a combination of transmission electron microscopy (TEM) and small-angle X-ray scattering (SAXS). To prepare thin sections for microscopy, a Leica Ultracut UCT microtome equipped with a Leica EM 
FCS cryogenic sample chamber operated at $-110^{\circ} \mathrm{C}$ was used to cut sections approximately $500 \AA$ in thickness. The sections were collected on TEM grids and stained four hours in $\mathrm{OsO}_{4}$ vapor. A JEOL 100CX TEM, operated at an accelerating voltage of $100 \mathrm{kV}$, was used to image the stained sections. SAXS data were collected at the Advanced Polymers Beamline (X27C), located at the National Synchrotron Light Source at Brookhaven National Labs (BNL), Upton, NY. Two-dimensional scattering patterns were collected on Fujitsu image plates, then read by a Fujitsu BAS 2000 image plate reader. Custom software at BNL was used to subtract background noise and perform circular averaging. Data were collected for a wavelength of $1.307 \AA$ and a camera length of $1410 \mathrm{~mm}$.

\subsection{Results}

The results of the morphological characterization for the three $\mathrm{I}_{5} \mathrm{~S}$ materials are listed in Table 4.1. Circularly averaged, $I$ vs. $q$ SAXS data for all three $\mathrm{I}_{5} \mathrm{~S}$ samples as well as the $\mathrm{I}_{5}-\mathrm{S}-\mathrm{I}_{5}$ sample are shown in Figure 4.2. The excellent long-range order exhibited by these samples is indicated by multiple higher order reflections. The $q_{n} / q^{*}$ ratio of $1, \sqrt{3}, \sqrt{4}, \sqrt{7}, \sqrt{9}, \sqrt{12}, \sqrt{19} \ldots$ indicate that $\mathrm{I}_{5} \mathrm{~S}-37$ and $\mathrm{I}_{5} \mathrm{~S}-46$ form hexagonal morphologies. Here $q^{*}$ is the scattering vector of the lowest angle primary scattering peak and $q_{n}$ is the series of all scattering vectors for which peaks are observed. For $\mathrm{I}_{5} \mathrm{~S}$ 58 and $\mathrm{I}_{5}-\mathrm{S}-\mathrm{I}_{5}-66$ the scattering vectors of the peaks occurring at integral multiples of $q^{*}$ indicating a lamellar morphology. A two-dimensional SAXS pattern collected for sample $\mathrm{I}_{5} \mathrm{~S}-46$ is shown in Figure 4.3. The remarkable long-range order of this sample results in up to 9 orders of single crystal-like reflections. Usually, single crystal-like 
long-range order is achieved by melt state shear alignment ${ }^{113,114}$ or roll-cast ${ }^{115,116}$ and is seldom achieved simply by solvent casting. From the SAXS data, the lamellar long periods of $I_{5} S-58$ and $I_{5}-S-I_{5}-66$ were found to be $49.7 \pm 0.5 \mathrm{~nm}$ and $45.2 \pm 0.5 \mathrm{~nm}$, respectively. The scattering vector ratios found from the SAXS on $I_{5} S-46$ and $I_{5} S-37$ were consistent with cylinders on a hexagonal lattice with (100) spacing of $52.1 \pm 0.5$ $\mathrm{nm}$ and $47.0 \pm 0.5 \mathrm{~nm}$, respectively. Shown in Figure 4.4 shows representative TEM micrographs, which confirm that $\mathrm{I}_{5} \mathrm{~S}-58$ and $\mathrm{I}_{5}-\mathrm{S}-\mathrm{I}_{5}-66$ form lamellar morphologies, and $\mathrm{I}_{5} \mathrm{~S}-46$, and $\mathrm{I}_{5} \mathrm{~S}-37$ form cylindrical morphologies..

\subsection{Discussion}

If we considered the dumbbell shaped $\mathrm{I}_{5}-\mathrm{S}-\mathrm{I}_{5}$ as a combination of two $\mathrm{I}_{5} \mathrm{~S}$ miktoarm stars, an approach used by several studies of multigraft block copolymer architectures, ${ }^{3,106,107}$ the $\mathrm{I}_{5}-\mathrm{S}-\mathrm{I}_{5}-66$ should have similar morphology to that of an $\mathrm{I}_{5} \mathrm{~S}$ with polystyrene volume fraction of $66 \%$, and an overall molecular weight of 101,500 $\mathrm{g} / \mathrm{mol}$. According to the previous study of Beyer, Gido, and co-workers ${ }^{8} \mathrm{I}_{5} \mathrm{~S}$ star block copolymers with $66 \%$ PS volume fraction should form a lamella morphology, in agreement with the observed lamella morphology of $\mathrm{I}_{5}-\mathrm{S}-\mathrm{I}_{5}-66$. The previous study reported a lamellar spacing of $42.7 \pm 0.5 \mathrm{~nm}$ for an $\mathrm{I}_{5} \mathrm{~S}-60$ block copolymer sample with 0.60 PS volume fraction and a molecular weight of $128,000 \mathrm{~g} / \mathrm{mol}$ (PS arm molecular weight of $76,000 \mathrm{~g} / \mathrm{mol}$ and PI molecular weight of $10,400 \mathrm{~g} / \mathrm{mol}$ per arm). The $I_{5} \mathrm{~S}$ unit of the $\mathrm{I}_{5}-\mathrm{S}-\mathrm{I}_{5}$ sample has a PS arm molecular weight of $54,000 \mathrm{~g} / \mathrm{mol}$ and PI arms molecular weight of $7,400 \mathrm{~g} / \mathrm{mol}$, both are smaller than that of $\mathrm{I}_{5} \mathrm{~S}-60$. Interestingly, the 
$\mathrm{I}_{5}-\mathrm{S}-\mathrm{I}_{5}-66$ sample has a lamellar spacing of $45.2 \pm 0.5 \mathrm{~nm}$, which is larger than that of $\mathrm{I}_{5} \mathrm{~S}-60$. The previous study ${ }^{8}$ also reported the lamellar spacing of $\mathrm{I}_{5} \mathrm{~S}-67$ with total molecular weight of $152,000 \mathrm{~g} / \mathrm{mol}(106,000 \mathrm{~g} / \mathrm{mol}$ for the PS arm and $9,700 \mathrm{~g} / \mathrm{mol}$ for each PI arm) to be $47.9 \pm 0.5 \mathrm{~nm}$. At basically the same composition the $\mathrm{I}_{5} \mathrm{~S}-67$ sample has $50 \%$ greater molecular weight than the corresponding half of the $I_{5}-S-I_{5}-66$ architecture, yet its lamellar spacing is only $6 \%$ larger. These comparisons indicate that the two junction point structure of $\mathrm{I}_{5}-\mathrm{S}-\mathrm{I}_{5}-66$ results in a larger domain spacing than its respective $\mathrm{I}_{5} \mathrm{~S}$ structural unit.

The three $\mathrm{I}_{5} \mathrm{~S}$ samples characterized in this paper are plotted on the morphology diagram in Figure 4.5 along with data from samples from four previous works involving $\mathrm{A}_{2} \mathrm{~B}, \mathrm{~A}_{3} \mathrm{~B}, \mathrm{~A}_{2} \mathrm{~B}_{2}$ and $\mathrm{A}_{5} \mathrm{~B}$ miktoarm star block copolymers ${ }^{8,9,87,90}$. Those samples found to have morphological behavior differing from their theoretically predicted morphologies are shaded. This figure reveals an increase in the frequency of discrepancies between predicted and observed behaviors with increasing molecular asymmetry. As indicated in Figure 4.5, the theory predicts that the three $\mathrm{I}_{5} \mathrm{~S}$ samples of this study should form spheres. Sample $\mathrm{I}_{5} \mathrm{~S}-58$ is found experimentally to form a lamellar morphology and both samples $\mathrm{I}_{5} \mathrm{~S}-46$ and $\mathrm{I}_{5} \mathrm{~S}-37$ are found to form cylinders of PS. This discrepancy is in agreement with the general trend observed in prior studies.

As with previous studies, samples that disagree with theoretical predictions exhibit morphologies that should occur at lower $\varepsilon$ for a given $\phi_{\mathrm{B}}$, or at a higher $\phi_{\mathrm{B}}$ for a given $\varepsilon$. For example, Beyer, Gido and co-workers ${ }^{8}$ found that their $\mathrm{I}_{5} \mathrm{~S}-60$ sample formed lamellae instead of the predicted cylinder of PS. While $\varepsilon$ for $\mathrm{I}_{5} \mathrm{~S}-60$ was 
calculated to be approximately 4.4 , for the same volume fraction, lamellae occur at a maximum of $\varepsilon \approx 3.0$. Alternately, for $\varepsilon=4.4$, cylinders are predicted to occur for $\phi_{13}$ greater than 0.59 , but are observed at $\phi_{\mathrm{B}}$ of 0.37 . If similar comparisons are made for all the samples that disagree with the theory, one finds that the overestimation of morphological shift increases with asymmetry; slight discrepancies at low values of $\varepsilon$ become greater as asymmetry increases. This is represented graphically in Figure 4.5, which shows a plot of $\Delta \varepsilon$ as a function of increasing $\varepsilon$, where $\Delta \varepsilon$ is the negative shift in $\varepsilon$ required to bring a sample into agreement with the diagram. The $\mathrm{I}_{5} \mathrm{~S}-37$ sample, for example, would have $\Delta \varepsilon$ of $4.4-2.5=1.9$. Plotted as a function of $\varepsilon$, the maximum of $\Delta \varepsilon$ increases with $\varepsilon$. These trends can only be represented approximately given the limited experimental data available, i.e. the data points available do not necessarily represent the limits of the discrepancy between theory and experiment at each value of $\varepsilon$.

Recently, Gido and co-workers ${ }^{9,106}$ and others ${ }^{117,118}$ have postulated that discrepancies between the Milner theory and experimental results, as illustrated in Figures 4.5 and 4.6, are a result of additional chain stretching inherent in the miktoarm star architecture due to crowding of multiple arms linked to a single junction point. Our current data suggests that, as architectural asymmetry (arm number asymmetry) becomes large, geometric packing constraints also exert an important influence on morphology. Milner's calculation was based on the assumption of a rounded WignerSeitz cell surrounding each domain. " In order for cylindrical and spherical domains to fill space they must occupy true, space filling polyhedral Wigner-Seitz cells and deform 
to fill the corners of these cells. ${ }^{83,119}$ Therefore, the energies calculated with the rounded cell approximation (upon which Figure 4.5 is based) represent lower bounds. When the volume fraction of the component, which forms the core of the cylinder or sphere, is small (as in diblock copolymers), packing problems are not very important. However, as the molecule becomes more and more asymmetric, the volume fraction of the multi-arm component on the convex side of the interface may decrease to the point where it approaches the volume fraction of the interstitial space in a close packing of spheres. For example, at $\varepsilon=5$ and $\phi=0.6$, the morphology diagram predicts a spherical morphology with the 0.6 volume fraction component inside the spheres and only 0.4 volume fraction in the matrix between spheres. However, the maximum volume fraction that solid spheres in a bec packing can occupy is only 0.65. Real spherical block copolymer domains will not be able to achieve or even closely approach the solid contact limit for packing density of the domain cores, due to the severe conformational distortions which would be required for the corona blocks. Thus we expect that geometric packing limits will prevent increasing shifts to higher $\phi$ of the true experimentally determined order-order transition lines in Figure 4.5 as $\varepsilon$ becomes large. A similar geometric packing limit will also apply to the cylindrical morphology. At these limits the order-order transitions will become vertical lines on the morphology diagram. On Figure 4.5, we have sketched the approximate positions, based on experimental data, of geometrical packing limits for spherical and cylindrical morphologies. 
In the lamella forming sample, $\mathrm{I}_{5} \mathrm{~S}-58$, unusual chevron tilt grain boundary morphologies were observed, as shown in Figure 4.6. Previous experimental ${ }^{120-123}$ and theoretical studies $^{124,125}$ of symmetric tilt grain boundaries in diblock copolymers have reported chevron tilt boundaries, as illustrated in Figure 4.7, in which lamellar layers retain their continuity while bending cooperatively in a narrowly defined boundary region. Figure 4.6 shows what we will call broken chevrons that occur in the $\mathrm{I}_{5} \mathrm{~S}$ material. In Figure 4.6a, the lamellac on both sides of the boundary terminate at the boundary in rows of semicylindrical end calps similat to those observed in T-junction tilt boundaries. ${ }^{120,126}$ In Figure 4.6b, some lamellate terminate at the boundary in semicylindrical end caps and additionally individual complete cylinders occupy the symmetry plane of the chevron boundary. We will call this type of boundary a broken chevron with cylinders. Previous work ${ }^{120,124,125}$ showed that at high tilt angles the lamella forming a chevron boundary develop protrusions parallel to the plane of the boundary resulting in $\Omega$ and extended- $\Omega$ variants of the chevron. The protrusions are necessary in order to fill space to constant density in the plane of the chevron grain boundary at high tilt angles. Figure $4.6 \mathrm{c}$ shows that the $\mathrm{I}_{5} \mathrm{~S}$ material forms a broken form of the $\Omega$-boundary with additional cylindrical domains in the plane of the boundary. Recently Qiaro and Winey ${ }^{123}$ have published TEM images of $\Omega$-boundaries in diblock copolymers formed in sheared samples which also contain some cylindrical domains localized in the plane of the boundaries. Unlike Figure 4.6c, the lamellac involved in Winey's chevrons remain continuous across the grain boundaries. 
We postulate that the discontinuous nature of chevron tilt grain boundaries in $\mathrm{I}_{5} \mathrm{~S}-58$ results from an asymmetry in the energy penalties for interfacial bending due to the asymmetry inherent in the $\mathrm{I}_{5} \mathrm{~S}$ molecular architecture. At 0.58 PS volume fraction this material forms flat lamellar interfaces at equilibrium. However, bending of these interfaces is necessary to form a chevron tilt grain boundary. As shown in Figure 4.9a, the standard chevron boundary involves equal degrees of interfacial curvature both toward and away from the five-arm PI side of the interface. Due to crowding inherent in packing five arms of PI on the same side of the interface and the fact that curving the interface toward the PI side will exacerbate chain stretching resulting from this crowding, it may be energetically more costly to bend the interfaces toward the PI domains than toward the PS domains in an $\mathrm{I}_{5} \mathrm{~S}$ architecture. By replacing the standard cheveron structure with a broken or discontinuous structure as illustrated in Figure $4.9 \mathrm{~b}$, it is possible to achieve the required tilt reorientation using only interfacial curvatures which put the five PI arms on the convex side of the interface. In order to do this, however, it is necessary to produce higher interfacial curvatures capable of generating semicylindrical end-caps on lamellae and isolated cylinders in the grain boundary plane. For this reason the broken chevron structures are only observed at relatively high tilt angles. For instance the tilt angles of the broken chevrons in Figure $4.7 \mathrm{a}$ and $\mathrm{b}$ are about $90^{\circ}$. Presumably, as tilt angle increases a transition occurs from continuous to discontinuous chevron structure at the point where the energy penalty for the highly unfavorable bending toward the five PI arms (Figure 4.9a) first exceeds the energy to form cylinders and cylindrical caps with the five PI arms on the convex side of the interfaces (Figure 4.9b). 


\subsection{Conclusions}

As seen in prior studies, the Milner theory for the morphology of miktoarm star block copolymers exhibits a systematic tendency to overestimate the effect of architectural asymmetry on morphology. This tendency becomes exaggerated with increasing asymmetry as in the $I_{5} S$ samples of this study. Here we report a vertical straightening of the order-order transition lines in the morphology diagram at high $\varepsilon$ due to packing constraints inherent in placing a relatively small volume fraction material in the matrix around microphase separated domains. New discontinuous chevron tilt grain boundary morphologies were observed in a lamella forming $\mathrm{I}_{5} \mathrm{~S}$ material. The discontinuity of these boundaries is thought to result from asymmetric energy penalties for bending of the lamellar interfaces. It may be more energetically costly to curve interface toward the five arm per molecule PI side of the interface than toward the single arm per molecule PS side of the interface. 
Table 4.1. Molecular and morphological characterization information for the three $\mathrm{I}_{5} \mathrm{~S}$ miktoarm stars and the dumbbell shaped $\mathrm{I}_{5}-\mathrm{S}-\mathrm{I}_{5}$ graft block copolymer.

\begin{tabular}{|c|c|c|c|c|c|c|}
\hline & & & $\mathrm{I}_{5}-\mathrm{S}-\mathrm{I}_{5}-66$ & $\mathrm{I}_{5} \mathrm{~S}-58$ & $\mathrm{I}_{5} \mathrm{~S}-46$ & $\mathrm{I}_{5} \mathrm{~S}-37$ \\
\hline \multirow{4}{*}{ PS arm } & $\mathrm{Mn}(\mathrm{g} / \mathrm{mol})$ & $\mathrm{MO}$ & 118000 & 105000 & 78000 & 53000 \\
\cline { 2 - 7 } & $\mathrm{M}_{\mathrm{w}} / \mathrm{M}_{\mathrm{n}}$ & $\mathrm{SEC}$ & 1.07 & 1.03 & 1.04 & 1.03 \\
\hline \multirow{5}{*}{ I arm } & $\mathrm{Mn}(\mathrm{g} / \mathrm{mol})$ & $\mathrm{MO}$ & 7400 & 16300 & 16300 & 16300 \\
\cline { 2 - 7 } & $\mathrm{M}_{\mathrm{w}} / \mathrm{M}_{\mathrm{n}}$ & $\mathrm{SEC}$ & 1.04 & 1.03 & 1.03 & 1.03 \\
\hline & $\mathrm{Mn}(\mathrm{g} / \mathrm{mol})$ & $\mathrm{MO}$ & 203000 & 180000 & 162000 & 133000 \\
\cline { 2 - 7 } & $\mathrm{Mw}(\mathrm{g} / \mathrm{mol})$ & LALLS & 223000 & 193000 & 173000 & 141000 \\
\cline { 2 - 7 } & $\mathrm{M}_{\mathrm{w}} / \mathrm{M}_{\mathrm{n}}$ & $\mathrm{SEC}$ & 1.09 & 1.01 .07 & 1.07 & 1.06 \\
\cline { 2 - 7 } & Mass \% PS & ${ }^{1} \mathrm{H}-\mathrm{NMR}$ & 69 & 61 & 49 & 40 \\
\cline { 2 - 7 } & Mass \% PS & UV/SEC & 67 & 60 & 47 & 37 \\
\cline { 2 - 7 } & Mass \% PS & calc & 65 & 58 & 48 & 40 \\
\cline { 2 - 7 } & Vol \% PS & & 66.1 & 57.8 & 45.6 & 36.8 \\
\cline { 2 - 7 } & $\begin{array}{c}2 \pi / q^{*} \\
( \pm 0.5 \mathrm{~nm})\end{array}$ & & 45.2 & 49.7 & 52.1 & 47.0 \\
\cline { 2 - 7 } & $\begin{array}{c}\text { Predicted } \\
\text { Morphology }\end{array}$ & & Lamella & Sphere & Sphere & Sphere \\
\cline { 2 - 7 } & $\begin{array}{c}\text { Observed } \\
\text { Morpholody }\end{array}$ & & Lamella & Lamella & Cylinder & Cylinder \\
\hline
\end{tabular}



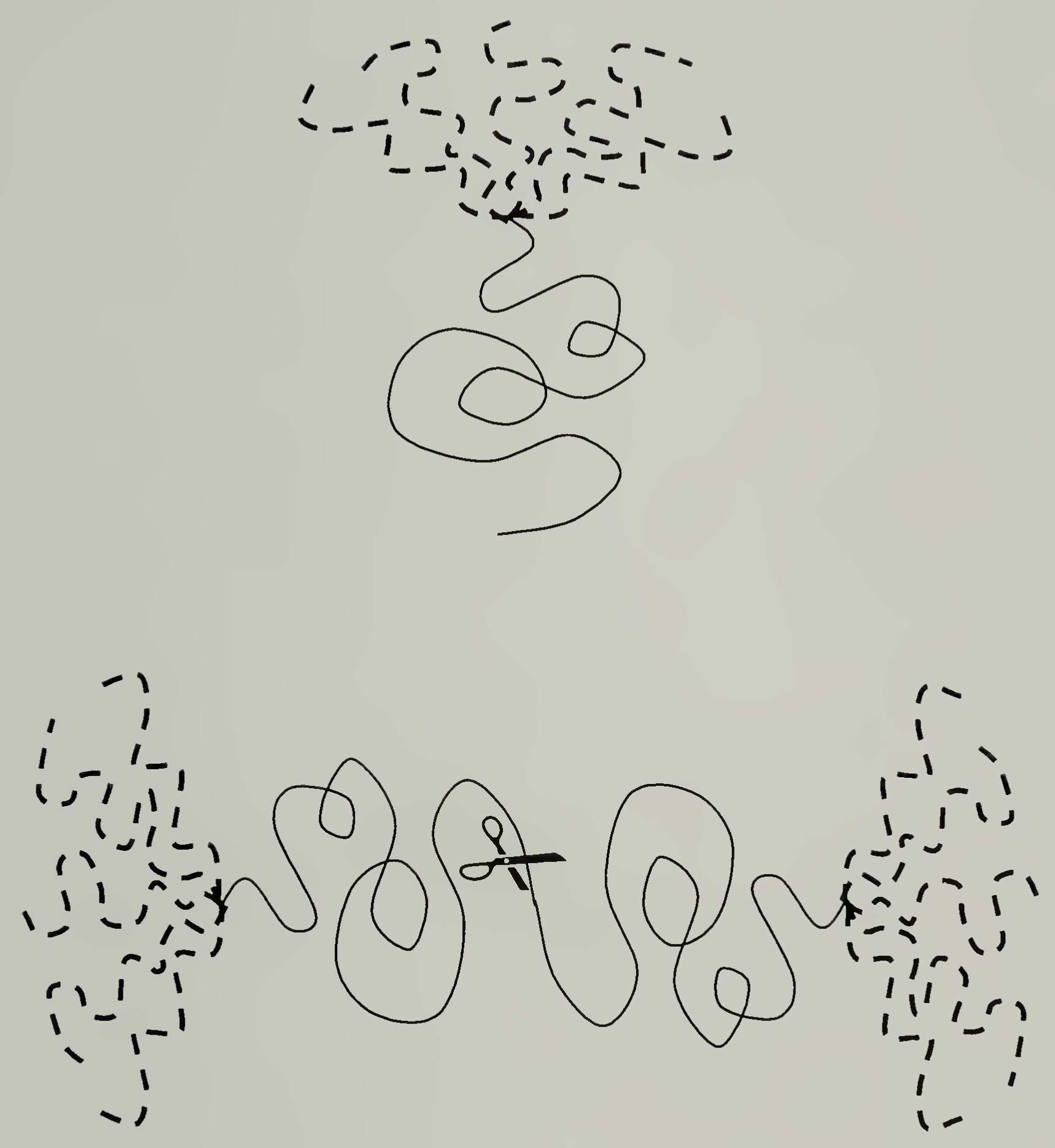

Figure 4.1. Illustration of the molecular architecture of an $\mathrm{I}_{5} \mathrm{~S}$ miktoarm star block copolymer and dumbbell shaped $\mathrm{I}_{5}-\mathrm{S}-\mathrm{I}_{5}$ graft block copolymer. 


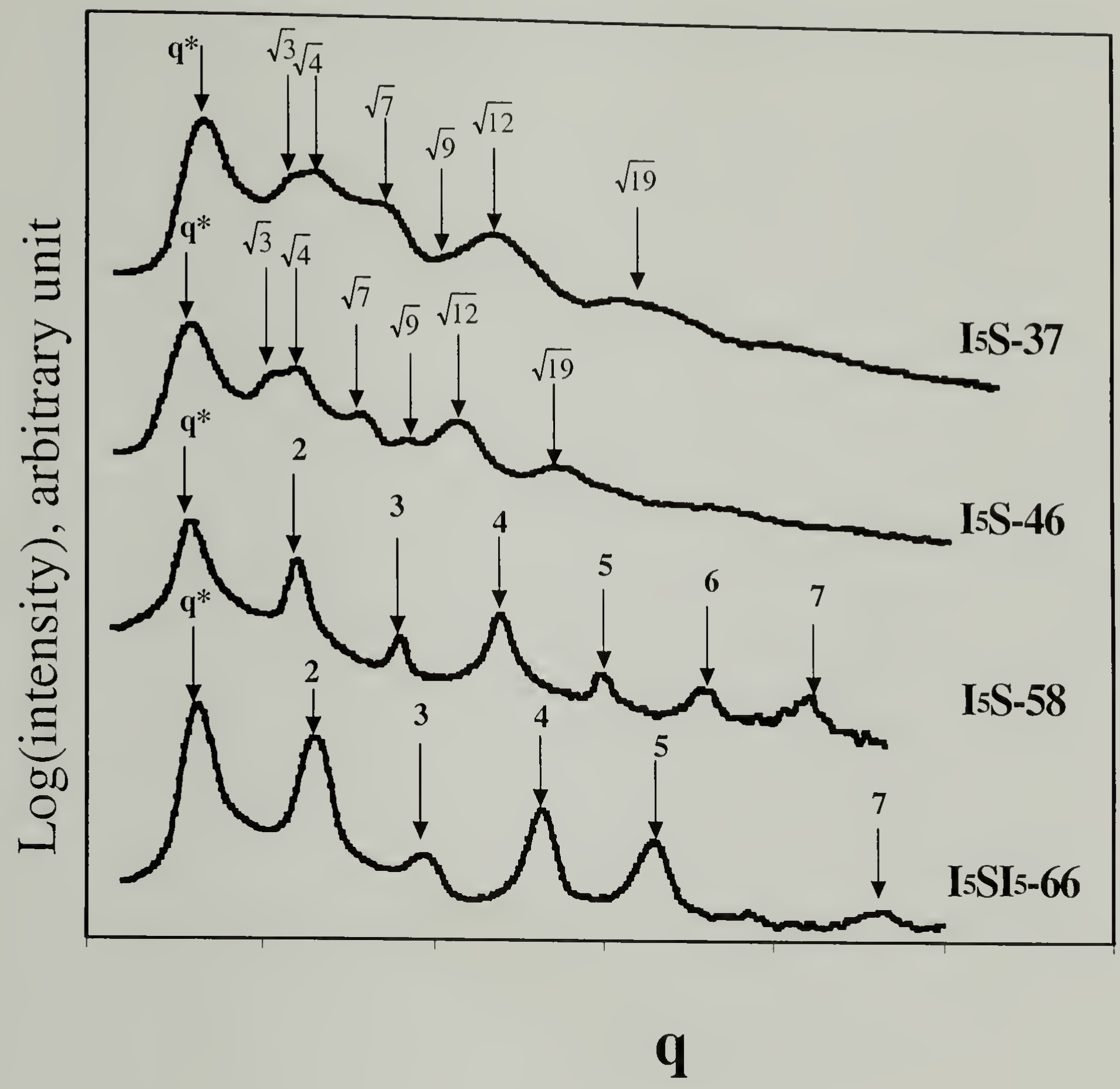

Figure 4.2. $\log (\mathrm{I})$ vs. q SAXS data for the $\mathrm{I}_{5} \mathrm{~S}$ and the $\mathrm{I}_{5}-\mathrm{S}-\mathrm{I}_{5}$ samples. 


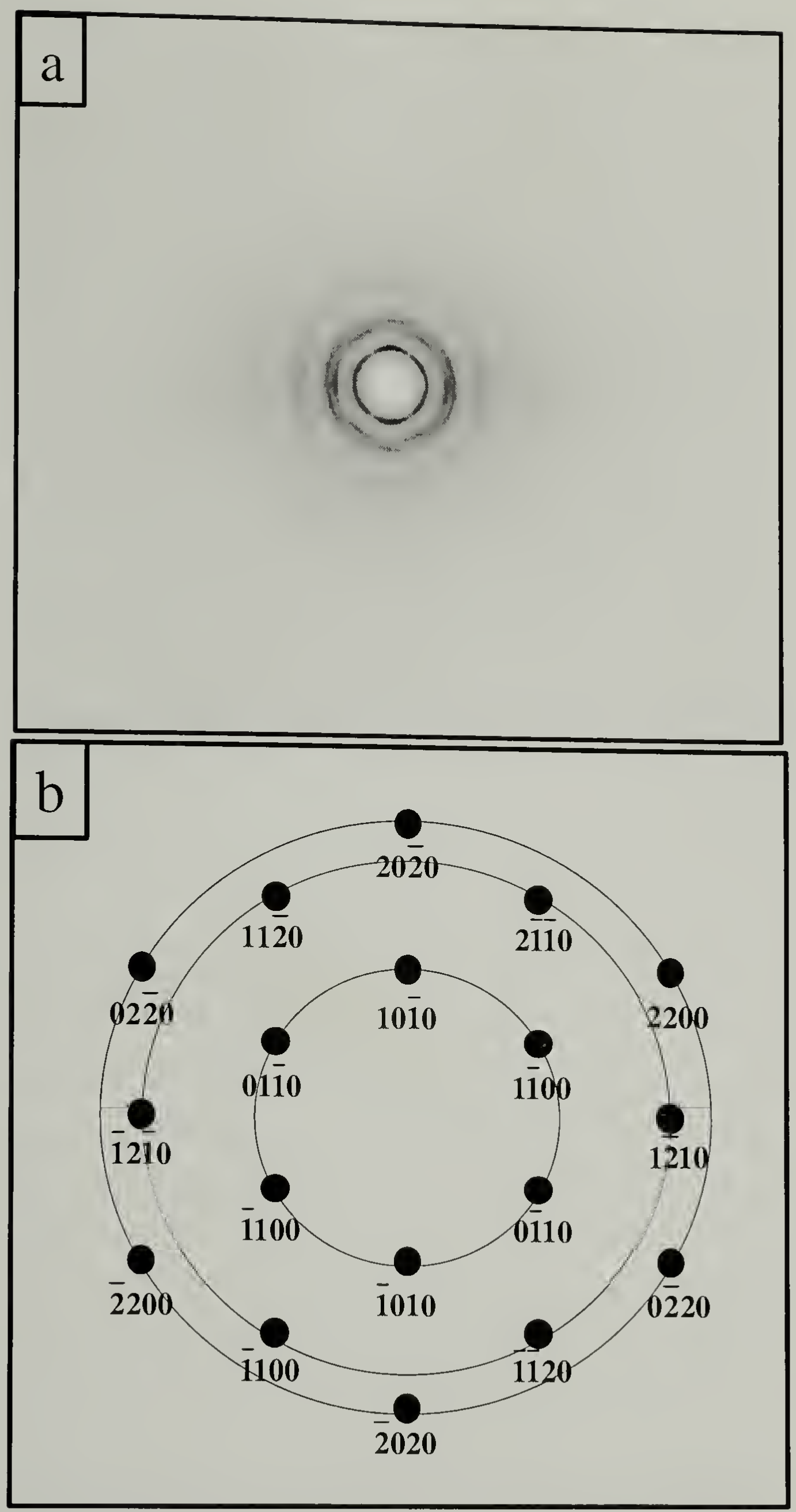

Figure 4.3. Two-dimensional SAXS for sample $\mathrm{I}_{5} \mathrm{~S}-46$ : (a) data, (b) indexed schematic. 


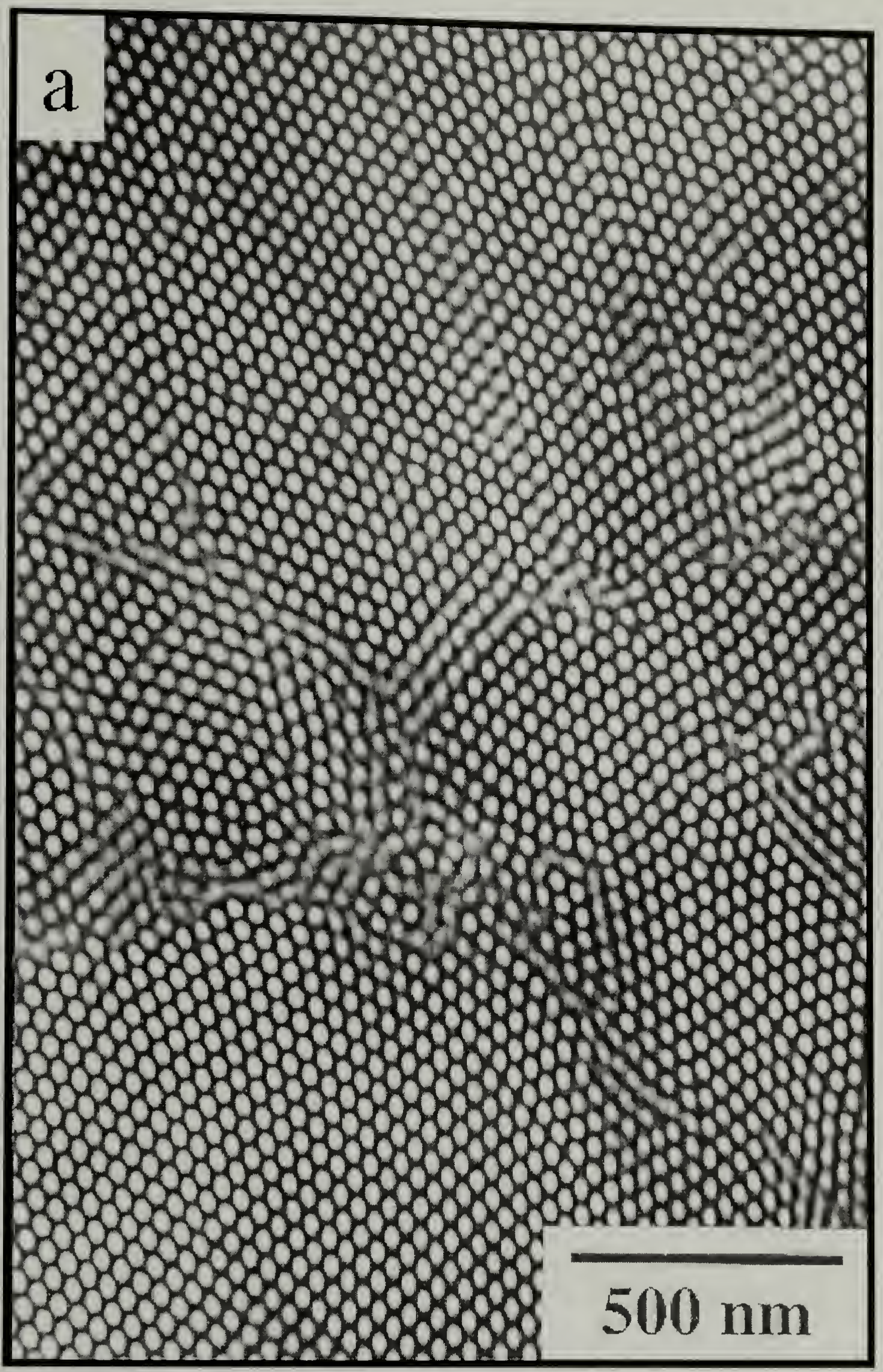




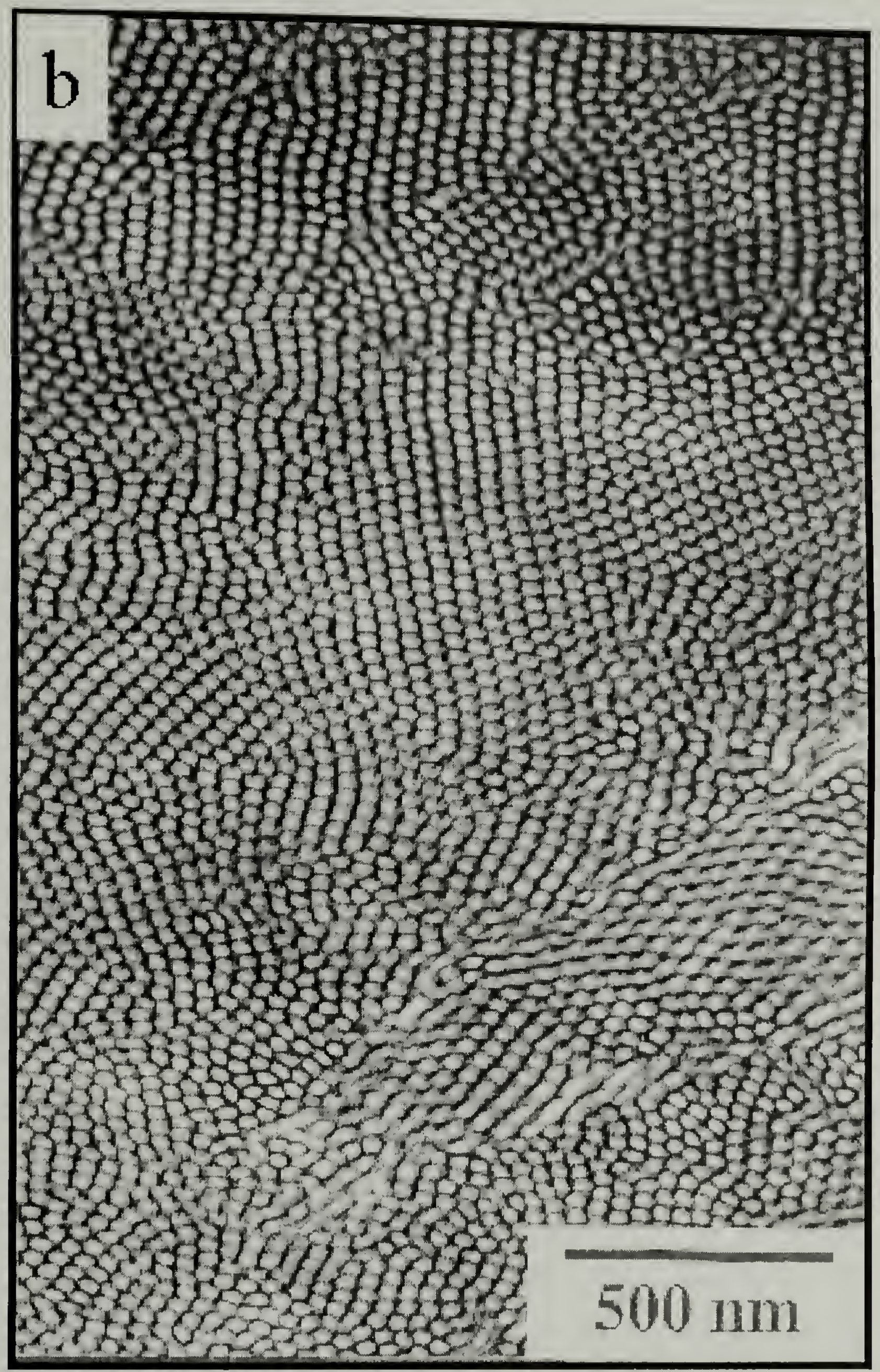




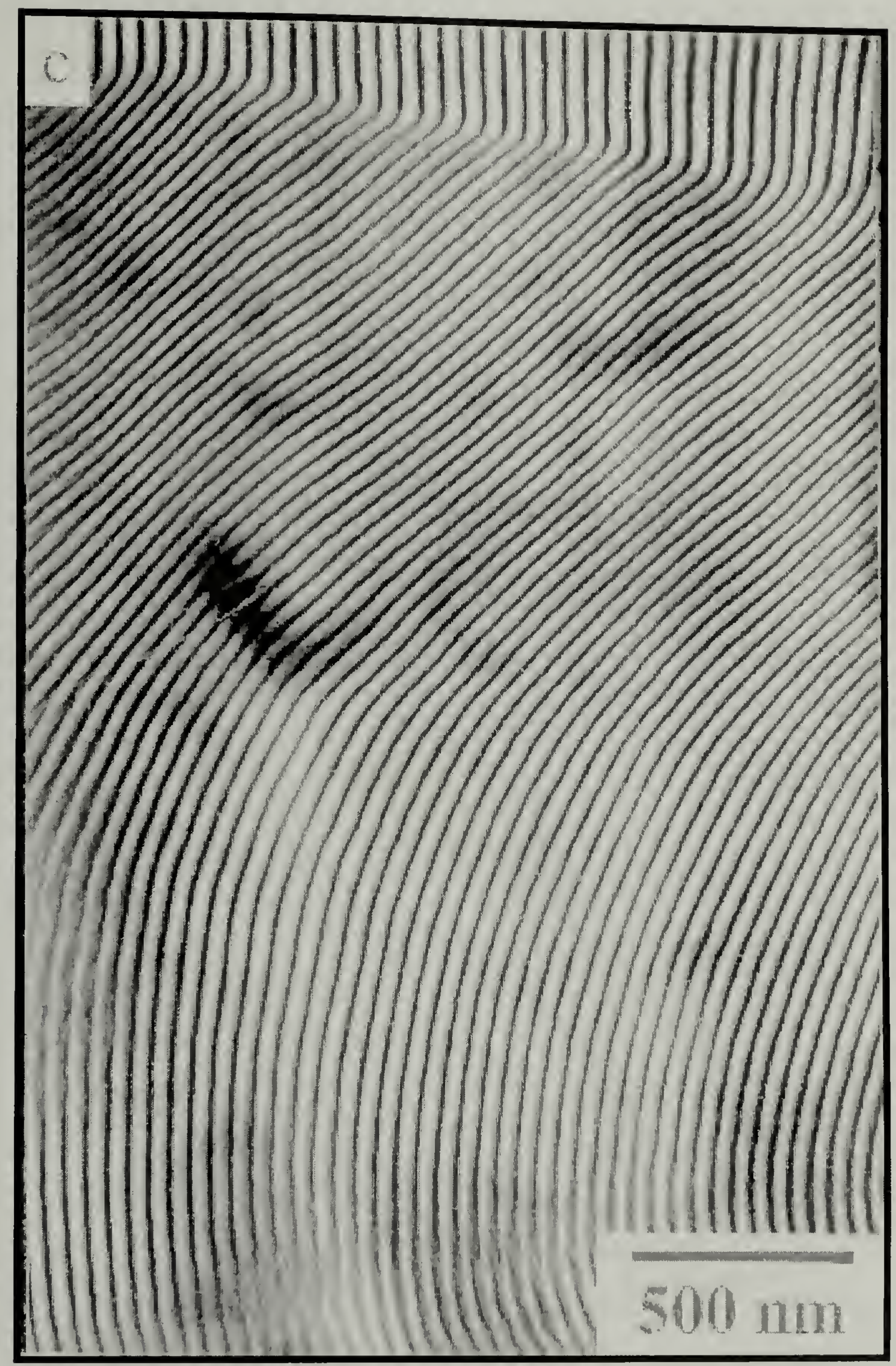




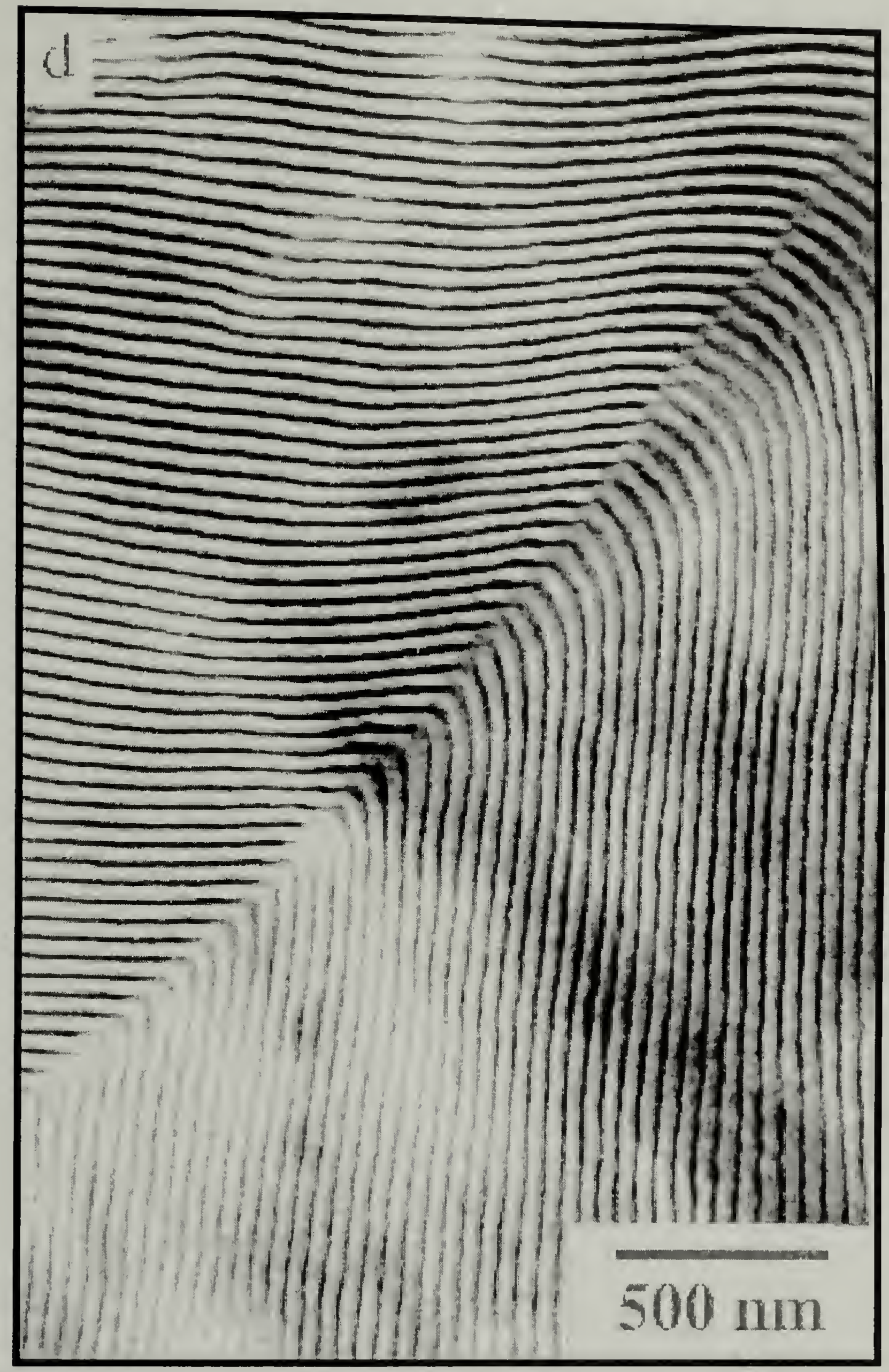

Figure 4.4. TEM micrographs of $\mathrm{I}_{5} \mathrm{~S}$ and $\mathrm{I}_{5}-\mathrm{S}-\mathrm{I}_{5}$ samples (a) $\mathrm{I}_{5} \mathrm{~S}-46$, (b) $\mathrm{I}_{5} \mathrm{~S}-37$, (c) $\mathrm{I}_{5} \mathrm{~S}-58$ and (d) $\mathrm{I}_{5}-\mathrm{S}-\mathrm{I}_{5}-66$. 


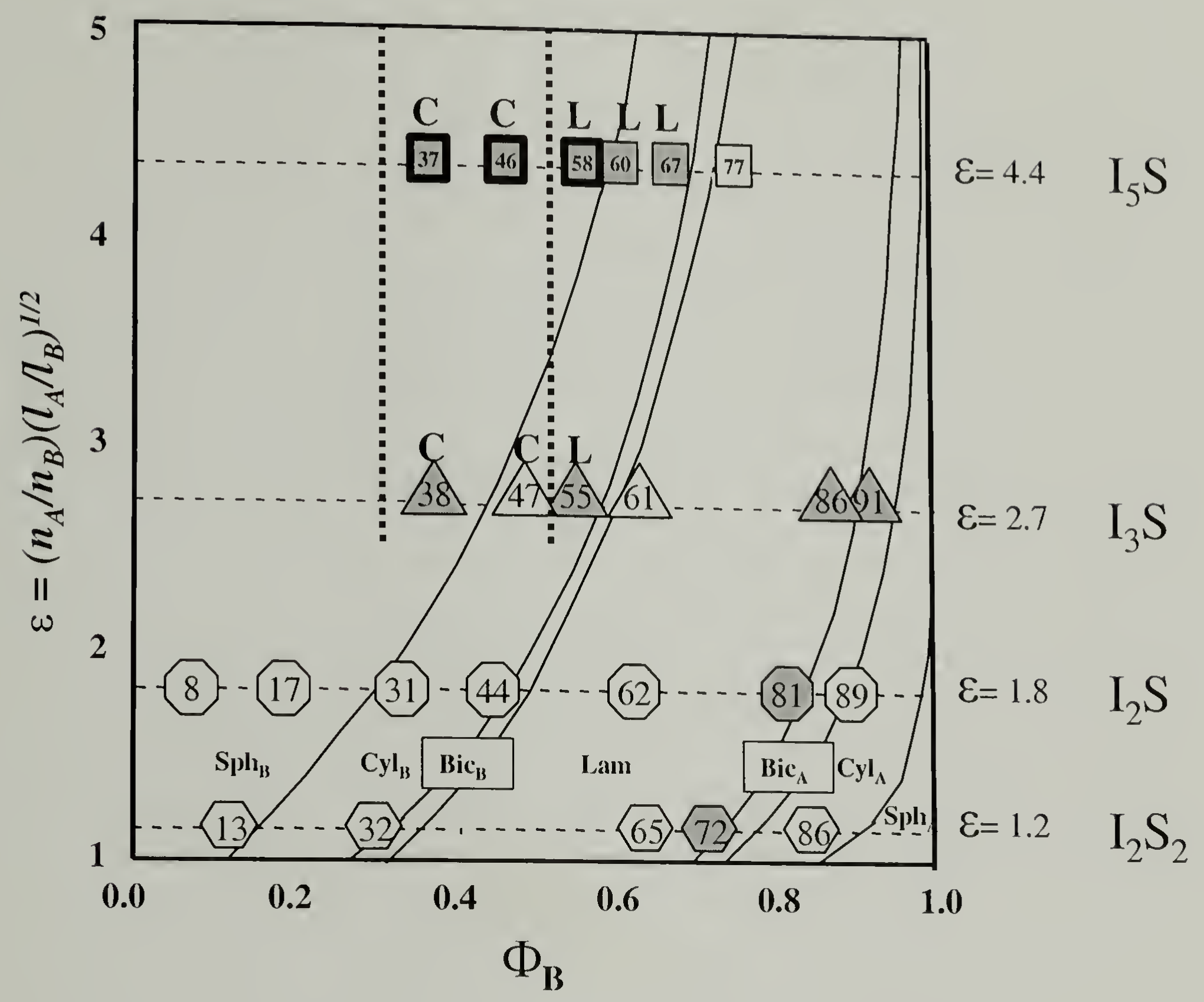

Figure 4.5. The morphology diagram generated by the Milner model. Morphology is given for the volume fraction of the $B$ component, $\phi_{B}$, and molecular asymmetry, $\varepsilon$. The $A_{5} B$ stars are represented by squares while $A_{2} B$ stars are represented by circles, $\mathrm{A}_{3} \mathrm{~B}$ stars by triangles, and $\mathrm{A}_{2} \mathrm{~B}_{2}$ stars by pentagons. Shaded symbols indicate samples whose morphologies disagree with that predicted by theory. Boldly outlined symbols indicate samples characterized in this study. 


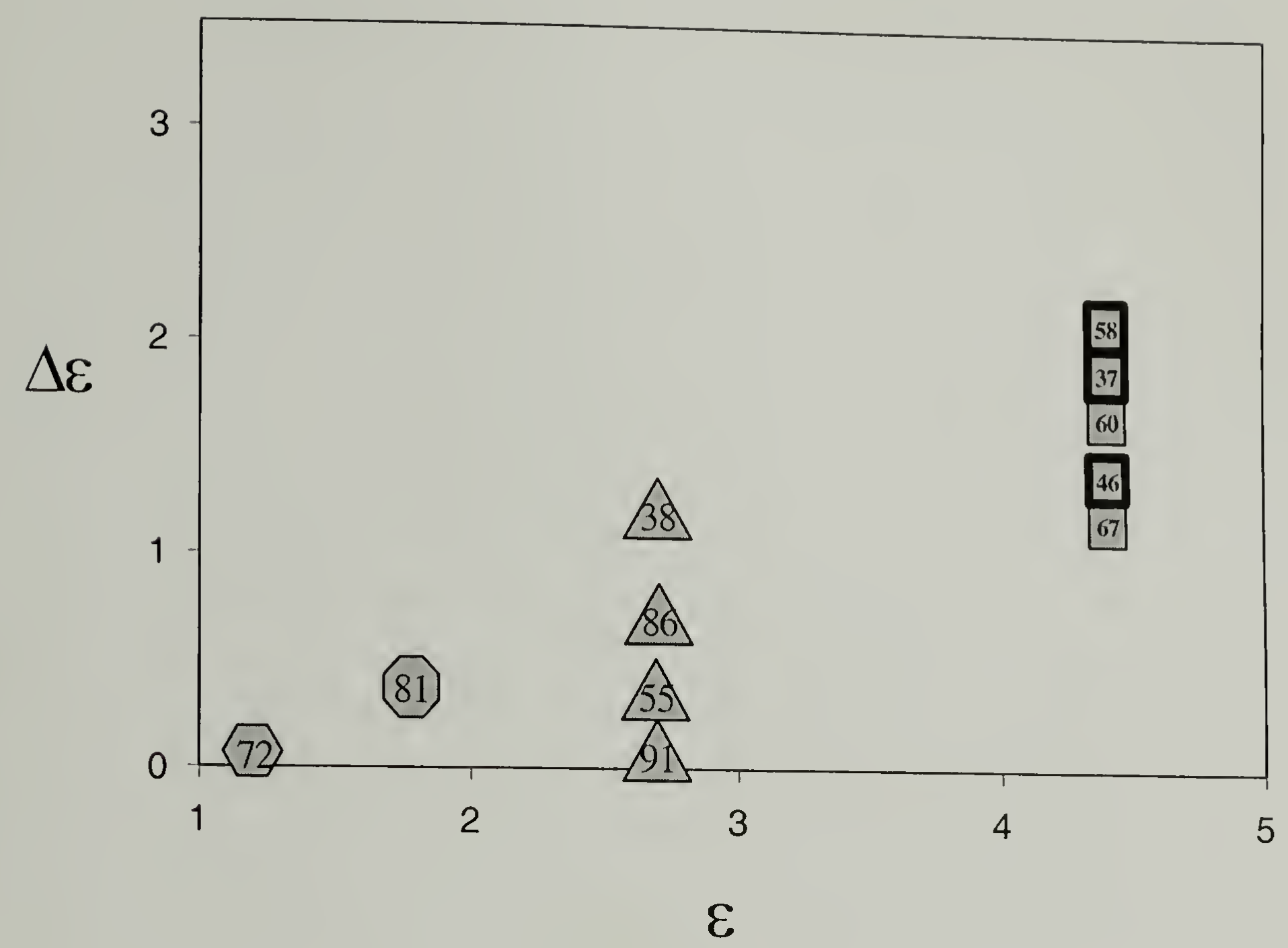

Figure 4.6. Values of $\Delta \varepsilon$ for samples exhibiting morphologies other than those predicted by theory, as a function of $\varepsilon$, for the data shown in Figure 4.5. Boldly outlined symbols indicate samples characterized in this study. 


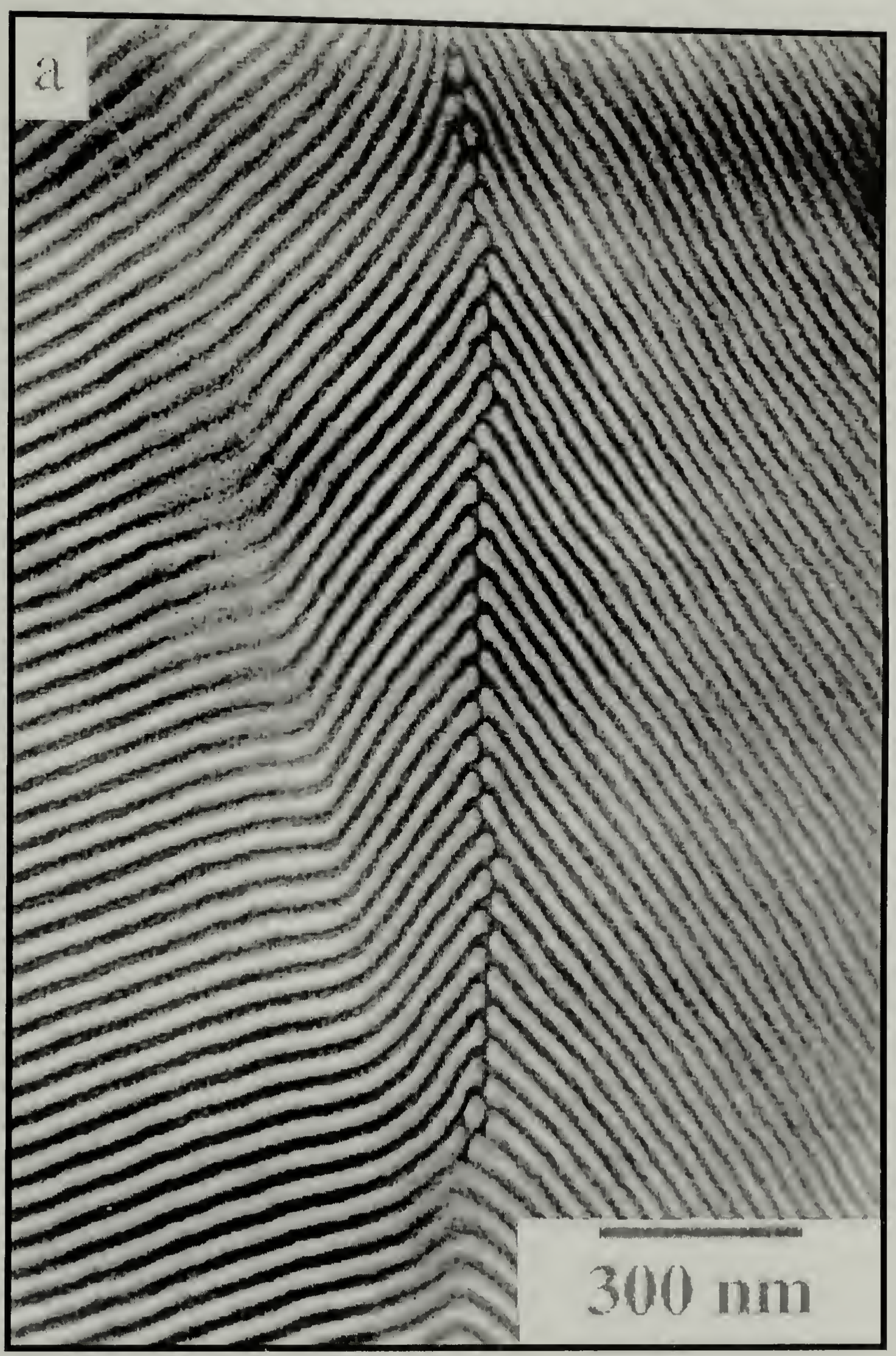




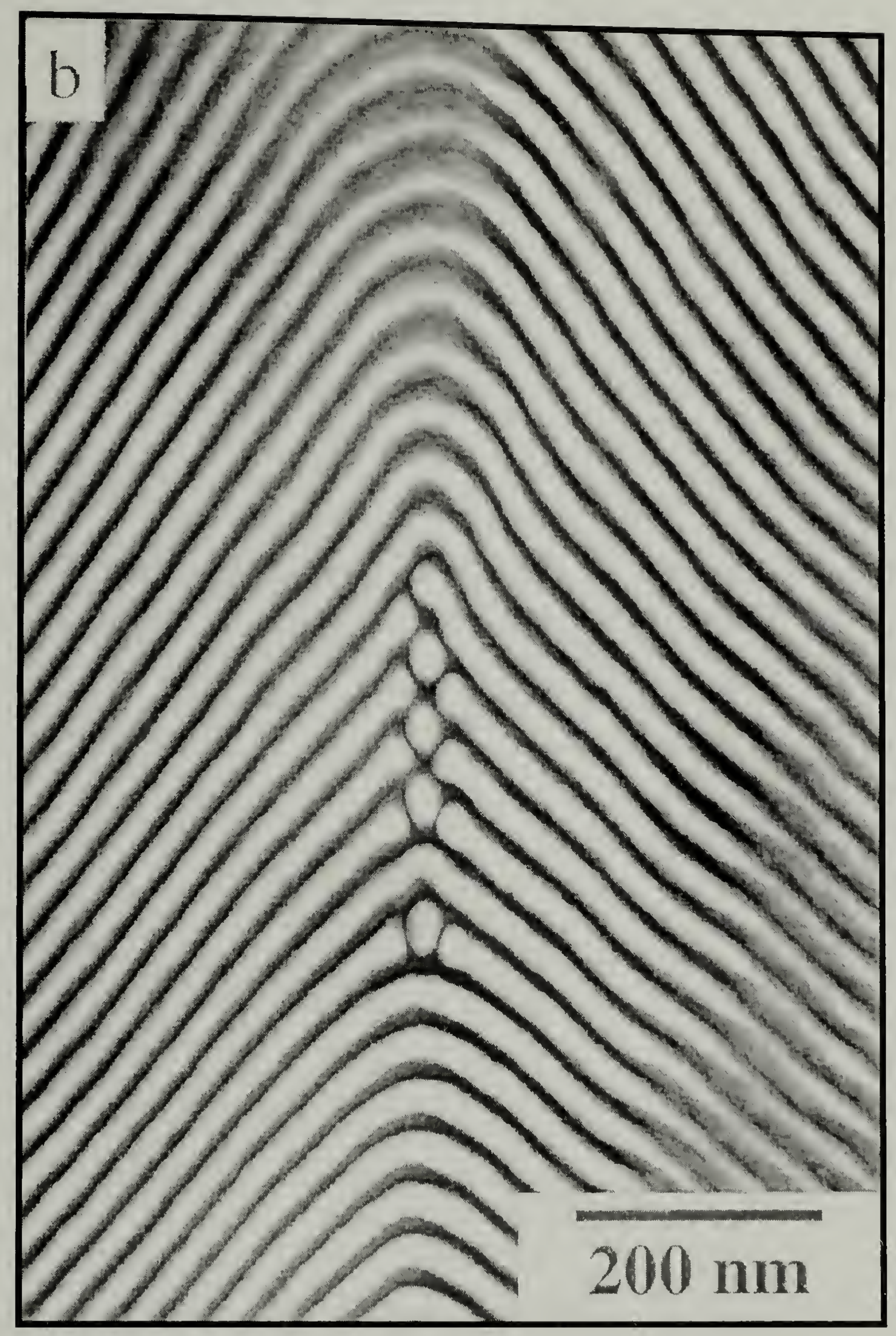




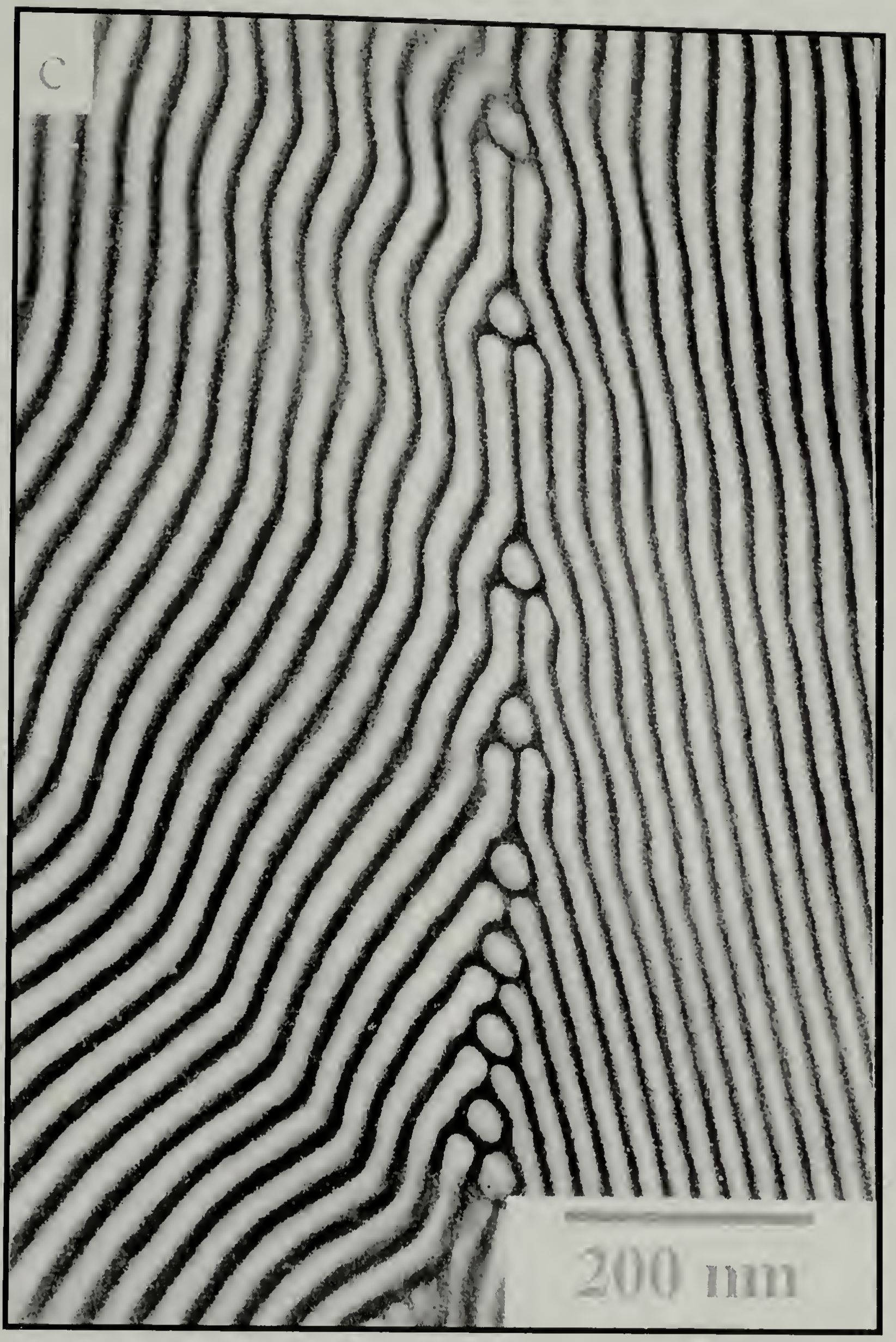

Figure 4.7. $\mathrm{I}_{5} \mathrm{~S}-58$ Symmetric tilt grain boundaries (a) broken chevron, (b) broken chevron with cylinders, (c) broken $\Omega$ with cylinder. 


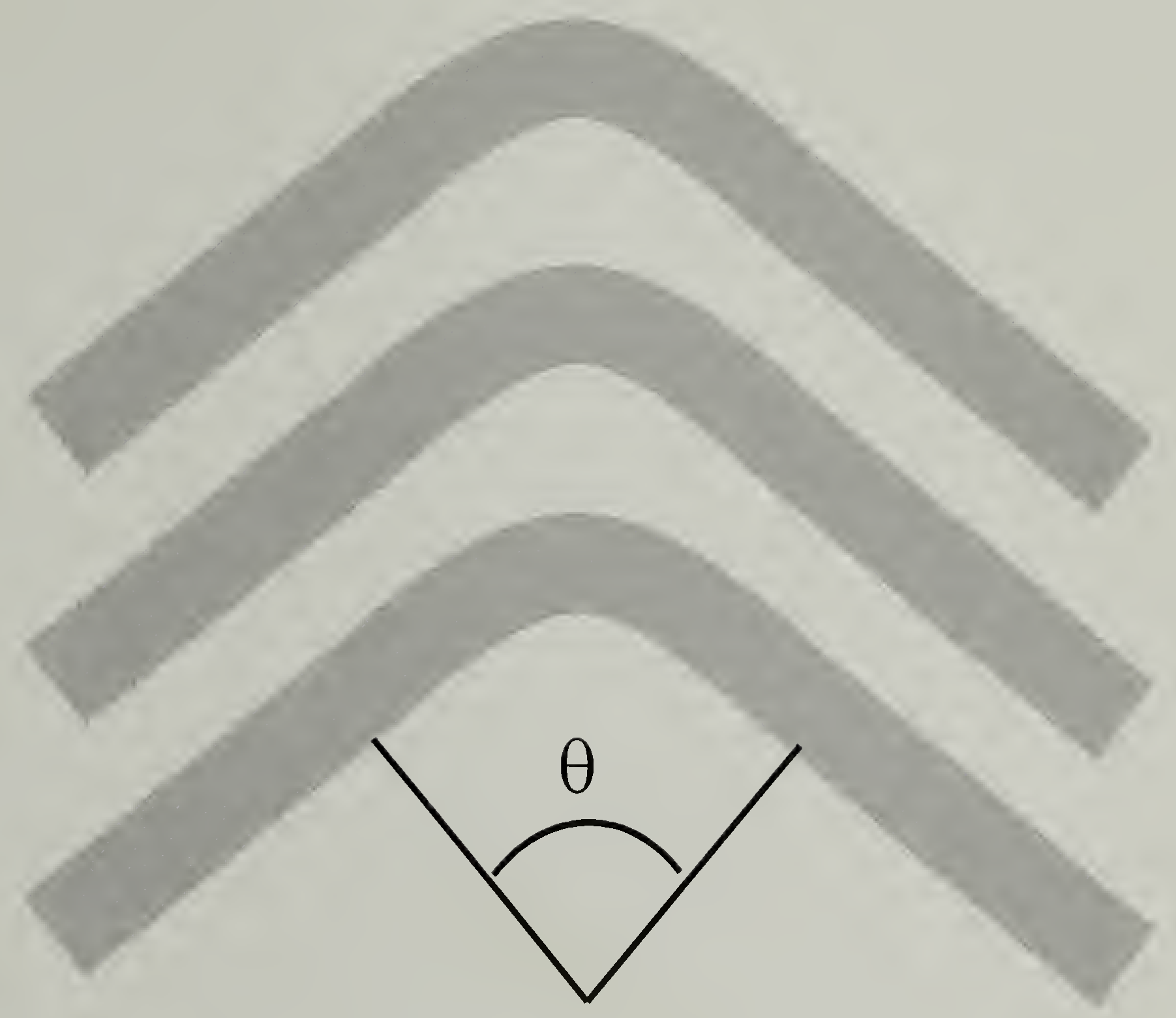

Figure 4.8. Schematic of commonly observed chevron tilt boundaries. 

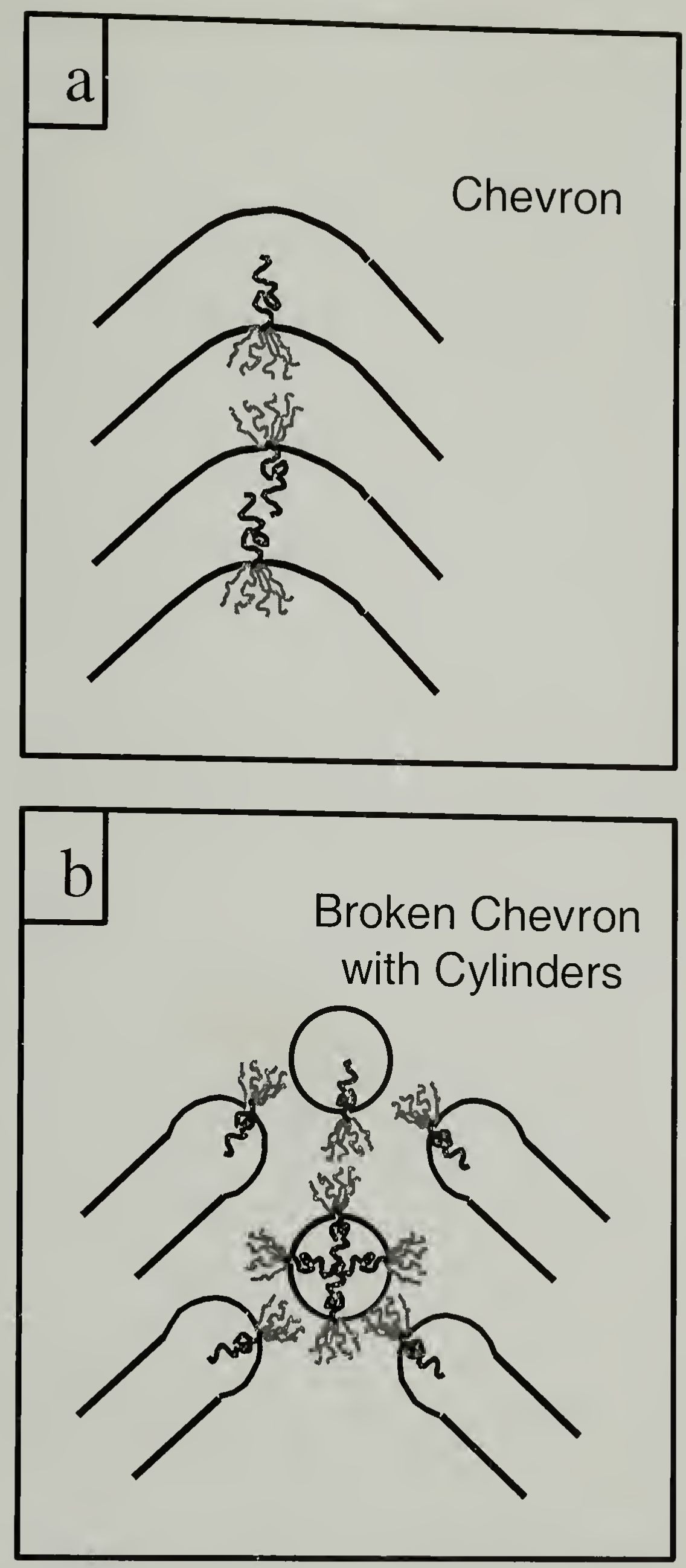

Figure 4.9. (a) illustration of chevron tilt boundaries for $\mathrm{I}_{5} \mathrm{~S}$ samples, (b) illustration of broken or discontinuous chevron tilt boundaries for $\mathrm{I}_{5} \mathrm{~S}$ samples. 


\section{CHAPTER 5}

MORPHOLOGICAL TRANSFORMATION FROM LAMELLA TO CYLINDER

\subsection{Abstract}

Casting from selective solvent cyclohexane, miktoarm star block copolymer $\mathrm{I}_{3} \mathrm{~S}$ 86 forms kinetically trapped, quasi-stable lamella morphology. $\mathrm{I}_{3} \mathrm{~S}-86$ has three polyisoprene arms and one polystyrene arm connected at a single junction point. Heated above $\mathrm{Tg}$, the selective solvent cast $\mathrm{I}_{3} \mathrm{~S}-86$ transforms from the kinetically trapped lamella morphology towards its stable cylinder morphology. The slow dynamics of the high molecular weight, non-linear $\mathrm{I}_{3} \mathrm{~S}-86$ block copolymer enable us to study the irreversible morphology transformation from lamella to cylinder at different intermediate stages. TEM analysis of these stages indicates that the perforated lamella morphology is an intermediate stage during lamella to cylinder transformation, and that the series of transformations from lamella to perforated lamella then to disordered worm structure occur within the original lamella planes.

\subsection{Introduction}

Selective solvent can be used to control block copolymers' morphologies. ${ }^{2,32,44,103}$ Thermal annealing of these kinetically trapped morphologies can provide us information about the path of transformations among these morphologies. Branched and grafted molecular architectures also have been shown to be an additional 
factor (in addition to volume fraction and degree of segregation) which affects the morphological behavior of block copolymers. Guided by the predictions of a mean field theory derived by Milner ${ }^{11,83}$ for the morphological behavior of $A_{n} B_{m}$-type, miktoarm star block copolymers, work has focused on molecules with an array of molecular architectures including $\mathrm{A}_{2} \mathrm{~B}$ stars and their homopolymer blends, ${ }^{1,754,87-90} \mathrm{~A}_{3} \mathrm{~B}$ stars, ${ }^{90}$ $\mathrm{A}_{\mathrm{n}} \mathrm{B}_{\mathrm{n}}$ stars and their blends with homopolymers, ${ }^{9,10,105,127} \mathrm{~A}_{5} \mathrm{~B}$ stars, ${ }^{8,102}$ multigraft architectures. $^{3,106,107}$ Branched architecture also slow down the dynamics of polymers. Both kinetic and thermodynamics play an important role on the morphologies of block copolymer systems. In this study, selective solvent and the slow dynamic of an $\mathrm{I}_{3} \mathrm{~S}$ miktoarm star block copolymer enable us to study the details of lamella to cylinder transformation.

The $\mathrm{I}_{3} \mathrm{~S}$ miktoarm star block copolymer used in the study consists of three equal length arms of polyisoprene (PI), and one arm of polystyrene (PS), linked at a common junction point. The molecular architectures of this material are illustrated in Figure 5.1. The $M_{n}$ of PI arm is $7,600 \mathrm{~g} / \mathrm{mol}$; the $M_{n}$ of PS arm is $180,000 \mathrm{~g} / \mathrm{mol}$; the total $M_{n}$ of the $\mathrm{I}_{3} \mathrm{~S}$ molecule is $204,000 \mathrm{~g} / \mathrm{mol}$. This particular $\mathrm{I}_{3} \mathrm{~S}$ material was calculated to have $86 \%$ PS in volume and is referred to as $\mathrm{I}_{3} \mathrm{~S}-86$. Milner's model predicted this sample to form a lamella morphology, however, a previous experimental study found the sample to form PI cylinder morphology. ${ }^{90}$

Hexagonally perforated lamellae, which resemble the cantenoid-lamellar structure derived by periodic area-minimizing surfaces calculations, ${ }^{48}$ were also observed recently in linear diblock systems $3^{35,37-39,46,49-52}$ in a narrow volume fraction 
region between HEX and LAM and in block copolymer / homopolymer blends. ${ }^{22.53,54}$ Controversy exists regarding some structure details of the perforated lamella structure and the stability of the morphology. Both $\mathrm{ABC}$ stacking of the hexagonally perforated layers $^{38,52}$ and a combination of an $\mathrm{ABC}$ and $\mathrm{AB}$ stacking ${ }^{39}$ were suggested for the perforated lamellar morphology. Regarding the stability of the morphology. The experimental studies by Hajduk et.al. suggest that perforated lamella is an unstable morphology involved in lamella to Gyroid transition. However, in a recent study by Yang, Gido and coworkers, perforated lamella was observed in a solvent cast blend sample of graft block copolymer and homopolymer, and the morphology persisted during thermal annealing. These observations suggest that perforated lamella can be stable or meta-stable. Using the methods of Semenov ${ }^{55}$ to estimate the free energy of perforated lamella in the strong segregation limit, Fredrickson ${ }^{56}$ showed that the perforated lamella is metastable with respect of cylinders and lamella at $\phi=0.35$. Qi and Wang $^{57-59}$ showed that the perforated lamellae appear as a "pseudostable" morphology during the lamellar to cylinder transition. However, this prediction has not been proved experimentally.

\subsection{Experimental}

The detailed synthesis and molecular characterization of the sample was reported previously. ${ }^{90}$ Bulk samples were prepared by dissolving the block copolymer in solution (5 weight percent) and then slowly evaporating the solvent over two weeks. Two solvents with different affinities for the two block materials were used: toluene, a non-selective solvent; cyclohexane, a selective solvent for PI. After getting solid cast 
films with approximately $1 \mathrm{~mm}$ thickness, residuc solvent was further removed from these films by holding them at room temperature in a vacuum oven for two weeks. Then, the samples were annealed in a vacuum oven at various temperatures and for various lengths of time.

The morphologies were characterized by transmission electron microscopy (TEM). To preparc thin sections for microscopy, a Leica Ultracut UCT microtome equipped with a Leica EM FCS cryogenic sample chamber operated at $-110^{\circ} \mathrm{C}$ was used to cut sections approximately $500 \AA$ in thickness. The sections were collected on TEM grids and stained four hours in $\mathrm{OsO}_{4}$ vapor. The PI microdomains are preferentially stained by $\mathrm{OsO}_{4}$, rendering them dark relative to PS microdomains due to mass-thickness contrast in the TEM. A JEOL 100CX TEM, operated at an accelerating voltage of $100 \mathrm{kV}$, was used to image the stained sections.

\section{$5.4 \quad$ Results}

I 3 S-86-A: Sample Cast From Toluene and anncaled at $120^{\circ} \mathrm{C}$ for two weeks.

After cast from toluene, a neutral solvent, $\mathrm{I}_{3} \mathrm{~S}-86$ forms ordered cylinder morphology (TEM micrograph no shown). This morphology persists during thermal annealing at $130^{\circ} \mathrm{C}$ for 2 weeks (TEM micrograph no shown). This observation agrees with a previous study by Hadjichristidis and coworkers. ${ }^{90}$

I 3 S-86-B: Sample Cast From Cyclohexane. 
When cast from cyclohexane, a selective solvent for polyisoprene, $\mathrm{I}_{3} \mathrm{~S}-86$ forms lamella morphology. A TEM micrograph of the lamella morphology is shown in Figure 5.2a. Because cyclohexane is selective for polyisoprene, it increases the effective volume fraction of polyisoprene and the block copolymer system form lamella morphology in concentrated solution. As the solvent evaporates, the $\mathrm{I}_{3} \mathrm{~S}$ material reaches its $\mathrm{Tg}$ and lost the mobility to rearrange itself into its stable morphologies, and the lamella morphology is kinetically trapped.

$\mathrm{I}_{3} \mathrm{~S}-86-\mathrm{C}$ : Sample $\mathrm{I}_{2} \mathrm{~S}-89-\mathrm{B}$ annealed at $110^{\circ} \mathrm{C}$ for 3 days.

After annealing $I_{2} S-89-B$ at $110{ }^{\circ} \mathrm{C}$ for 3 days the morphology of cyclohexane cast $\mathrm{I}_{3} \mathrm{~S}-86$ showed some interesting changes. A typical TEM micrograph is shown in Figure 5.2b. In the image, we can still sec the structure feature of alternating PS PI layer. However, the dark PI layers were perforated by PS materials as indicated by the white "holes" in the dark PI layers.

$\mathrm{I}_{3}$ S-86-D: Sample $\mathrm{I}_{3} \mathrm{~S}-86-\mathrm{C}$ further annealed at $120^{\circ} \mathrm{C}$ for 7 days.

After annealing the sample $I_{3} S-86-C$ at $120^{\circ} \mathrm{C}$ for a week, further structural evolution was observed. A TEM Tilting scries of this sample is shown in Figure 5.3. From the TEM micrographs, we can see, after tilting 20 degree around the indicated tilting axis, the perforated structures near the center of Figure 5.3a becomes a regular side projection of perforated lamella, which is an indication that the basic alternating PS PI layer structure is still maintained, the perforation process continues in the PI layers. The TEM micrographs also showed that the general sizes of the PS perforations in the PI layers of $I_{3} S-86-D$ are bigger than that of the $I_{3} S-86-C$. Due to the high PS 
volume fraction of 0.86 , the PI material adopts a mesh-like structure surrounding PS perforations. The PS perforation is generally hexagonally distributed in the PI layers.

$\mathrm{I}_{3} \mathrm{~S}-86$-E: Sample $\mathrm{I}_{3} \mathrm{~S}-86-\mathrm{D}$ further annealed at $140^{\circ} \mathrm{C}$ for one week.

The PI mesh structure further changed during the annealing. A TEM Tilting series of this sample is shown in Figure 5.4. Figure 5.4a is a typical TEM micrograph of $\mathrm{I}_{3} \mathrm{~S}$-86- E. It shows an irregular PI mesh structure. That indicates that the relative regular PI mesh structure in $I_{3} S-86-D$ is partially broken during the annealing. However, after tilting the area in microscope along the tilt axis for 40 degrees, we still observe that all the dark material (PI) still occurs within a plane. The basic structure of alternating PI PS layers is still maintained, although the PI material in "PI layer" is a actually disordered mesh, perforated by PS material. This indicates the breakup of the PI mesh structure is still in plane.

$\mathrm{I}_{3} \mathrm{~S}$-86-F: Sample $\mathrm{I}_{3} \mathrm{~S}-86-\mathrm{E}$ further annealed at $160^{\circ} \mathrm{C}$ for 3 days.

After annealing $I_{3} S-86-E$ at $160{ }^{\circ} \mathrm{C}$ for 3 days, the PI mesh structures further break up. A TEM Tilting series of this sample is shown in Figure 5.5. Figure 5.5a is a typical TEM micrograph of $\mathrm{I}_{3} \mathrm{~S}-86-\mathrm{F}$. It shows that most polyisoprene material form disordered and worm-like structures. Some worms connect with each other. The interconnected disordered mesh network observed in $I_{3} S-86-F$ further breaks up during the annealing at $160^{\circ} \mathrm{C}$. Figure $5.5 \mathrm{~b}$ is the TEM micrograph after we tile the area in Figure 5.5a along the indicated tilting axis for 30 degrees in microscope. It still shows the dark PI material occurs in lines in the image, which indicates that the worm-like 
structure formed by the PI materiat is still in-plane. However, the transformation is not clear cut, TEM projections similar to that of $I_{33} S-86-E$ are also observed in microscope.

I 3 S-86-G: Sample $\mathrm{I}_{3} \mathrm{~S}-86-\mathrm{F}$ further annealed at $180^{\circ} \mathrm{C}$ for 3 days.

After annealing $I_{3} S-86-E$ at $180{ }^{\circ} \mathrm{C}$ for 3 days, the material forms ordered cylinder morphology. A TEM micrograph of this sample is shown in Figure 5.6. This morphology is as same as the morphology of $I_{3,} S-86-A$.

\subsection{Discussion}

In melt entangled state, the branched architecture slows down the dynamic of Star-branched polymer dramatically. ${ }^{128}$ In this experimental study, the slow kinetics of the high molecular weight non-linear polymer cnable us to track the morphology transformations in detail. Agree with the previous study, ${ }^{90}$ the sample $\mathrm{I}_{3} \mathrm{~S}-86$ form cylinder morphology after casting from neutral solvent, and the morphology is stable during annealing, which indicates that cylinder morphology is the stable morphology for $I_{3} S-86$.

Casting from cyclohexanc, a selective solvent for PI, $\mathrm{I}_{3} \mathrm{~S}-86-\mathrm{B}$ forms regular lamella morphology. This morphology is kinetically trapped and is not stable when heated above $\mathrm{T}_{\mathrm{g}}$. During thermal annealing, the unstable lamella morphology goes though a series of changes towards its stable morphology. In Figure 5.7, we summarize the morphology transformations based on the TEM observations. First, PS perforated PI plane hexagonally, the perforation increase in size and the PI layers form mesh 
structures. During further anncaling at higher temperature, the mesh structures gradually break up, from the regular mesh structure first and then to irregular mesh structure and finally to disorder worms. All these transformations occur within the original lamella planes. After annealing at $180^{\circ} \mathrm{C}$ for 3 days, the material form ordered cylinder morphology, which is the same morphology as the toluene cast $\mathrm{I}_{3} \mathrm{~S}-86$.

The experiment observation clearly demonstrated that perforated lamella is an intermediate stage during lamella to cylinder transformations. This observation is supplement to the previous results showing that perforated lamella is long-lived nonequilibrium morphology involved in lamella to Gyroid transition, ${ }^{35.49}$ and is in agrecment with the theoretical prediction of Qi and Wang. ${ }^{57.59}$ However, the fact that perforated lamella occurs cluring the transitions from lamella to eylinder or from lamellat to gyroid does not rule out that perforated lamella can be stable or meta-stable at certain compositions. As shown in a recent study of Yang, Gido and coworkers, perforated lamella was found simply by solvent casting of a blend sample, which consists of graft block copolymer and homopolymer, and was found stable during thermal anncaling.

\subsection{Conclusions}

Morphology transition from cylinders to lamellac was studied via TEM. It was found that lamella morphology is not stable in the selective solvent cast $\mathrm{I}_{3} \mathrm{~S}-86$, and it transforms into its thermodynamically stable lamellac on annealing. The transition turned out to occur though a series of in-plane transformations: perforated lamella, PI 
mesh, and PI worms. This study shows that perforated lamella not only involves in the lamella to Gyroid transitions $35,37-39,46,49,50$ but also in lamella to cylinder transitions. 


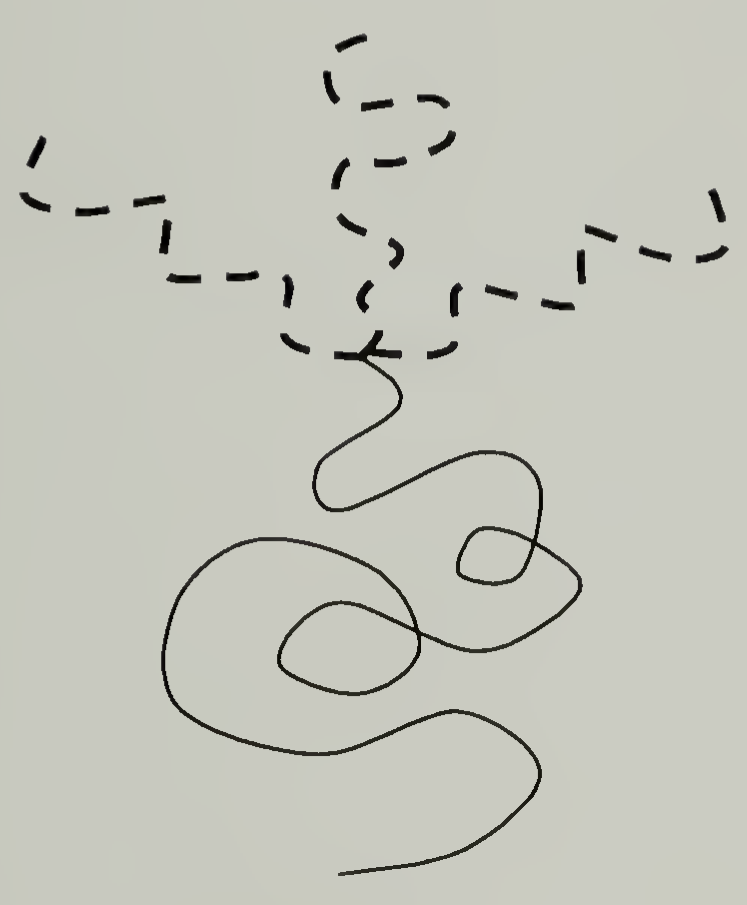

Figure 5.1. Illustration of the molecular architecture of an $\mathrm{I}_{3} \mathrm{~S}$ miktoarm star block copolymer. The $\mathrm{I}_{3} \mathrm{~S}$ star is comprised of three PI arms and one PS arm joined at a single junction point. 


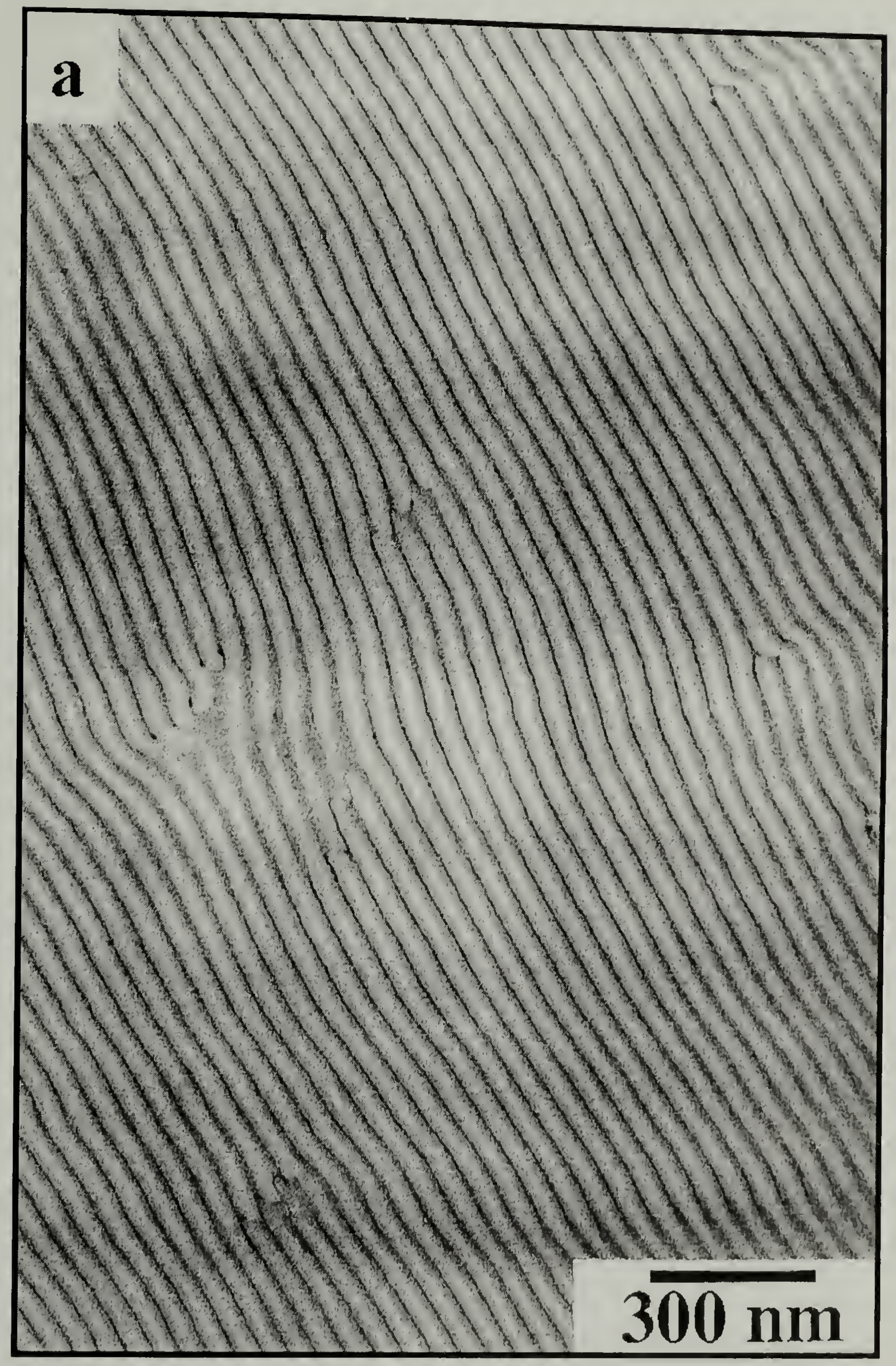




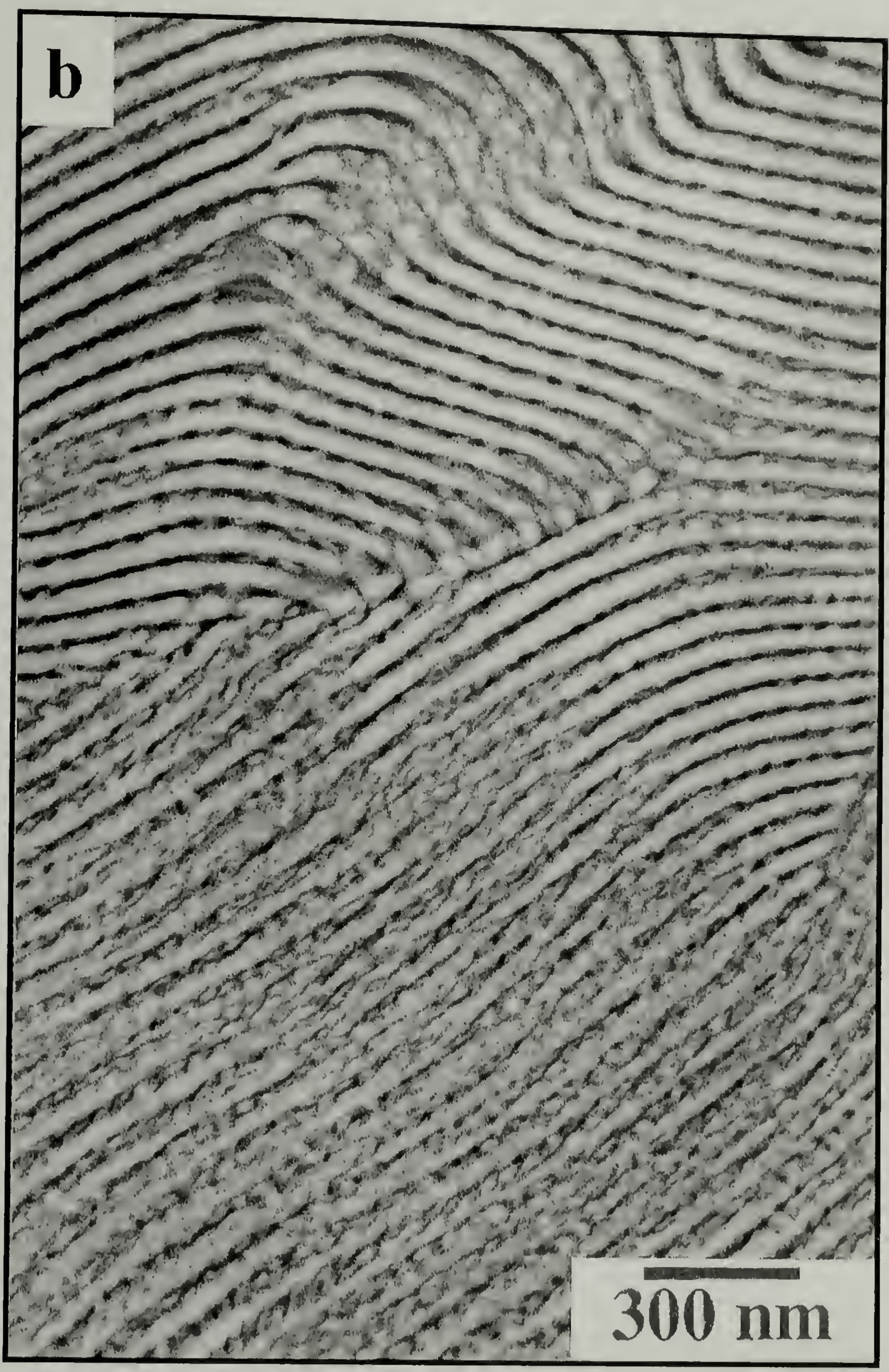

Figure 5.2. TEM micrographs of $\mathrm{I}_{3} \mathrm{~S}-86-\mathrm{B}$ and $\mathrm{I}_{3} \mathrm{~S}-86-\mathrm{C}$ : (a) $\mathrm{I}_{3} \mathrm{~S}-86-\mathrm{B}$; (b) $\mathrm{I}_{3} \mathrm{~S}-86-$ C. 


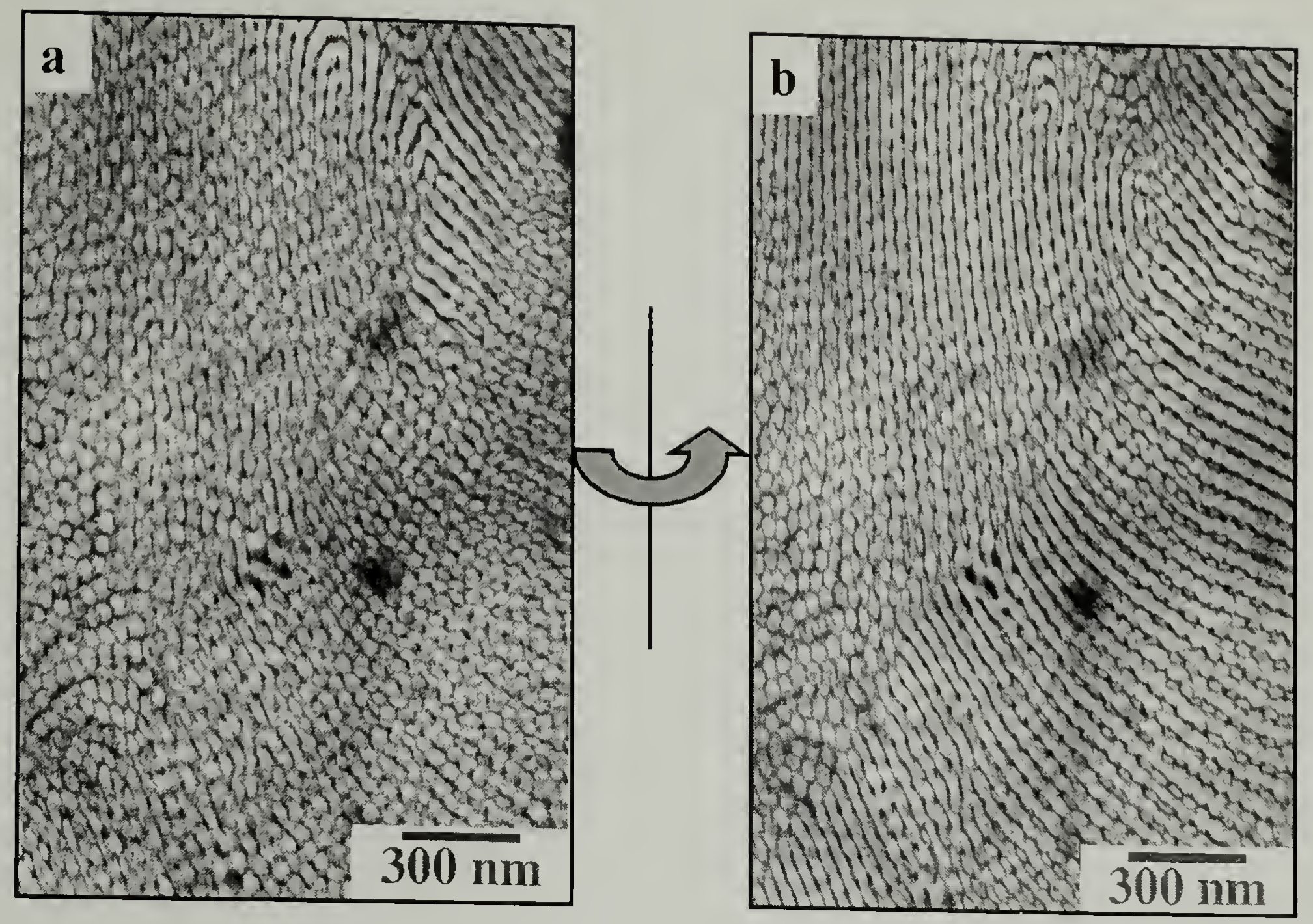

Figure 5.3. TEM tilt series of $\mathrm{I}_{3} \mathrm{~S}-86-\mathrm{D}$ (a) $0^{\circ}$ tilt. (b) $20^{\circ}$ tilt about axis indicated. 


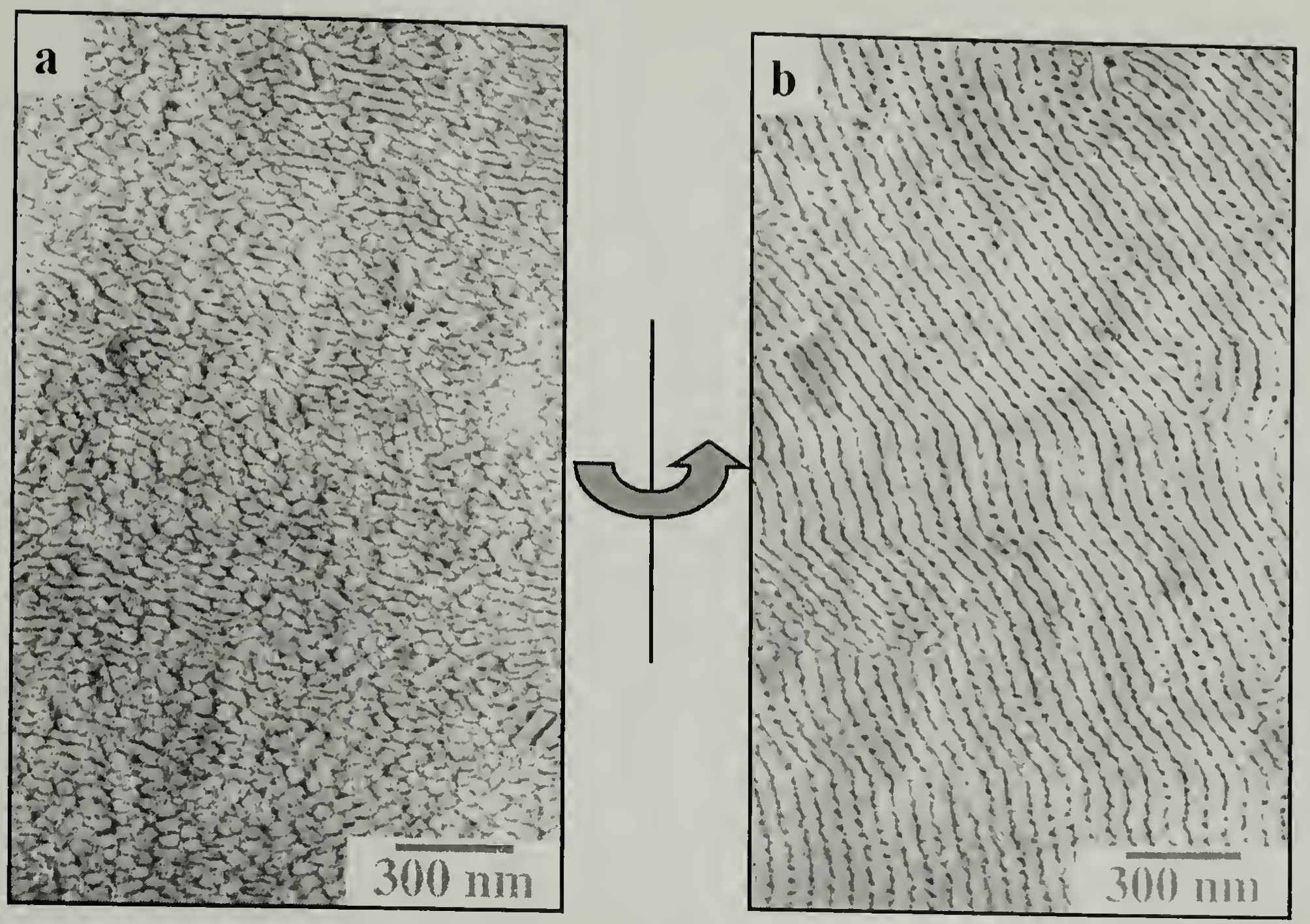

Figure 5.4. TEM tilt series of $\mathrm{I}_{3}$ S-86-E. (a) $0^{\circ}$ tilt. (b) $40^{\circ}$ tilt about axis indicated. 


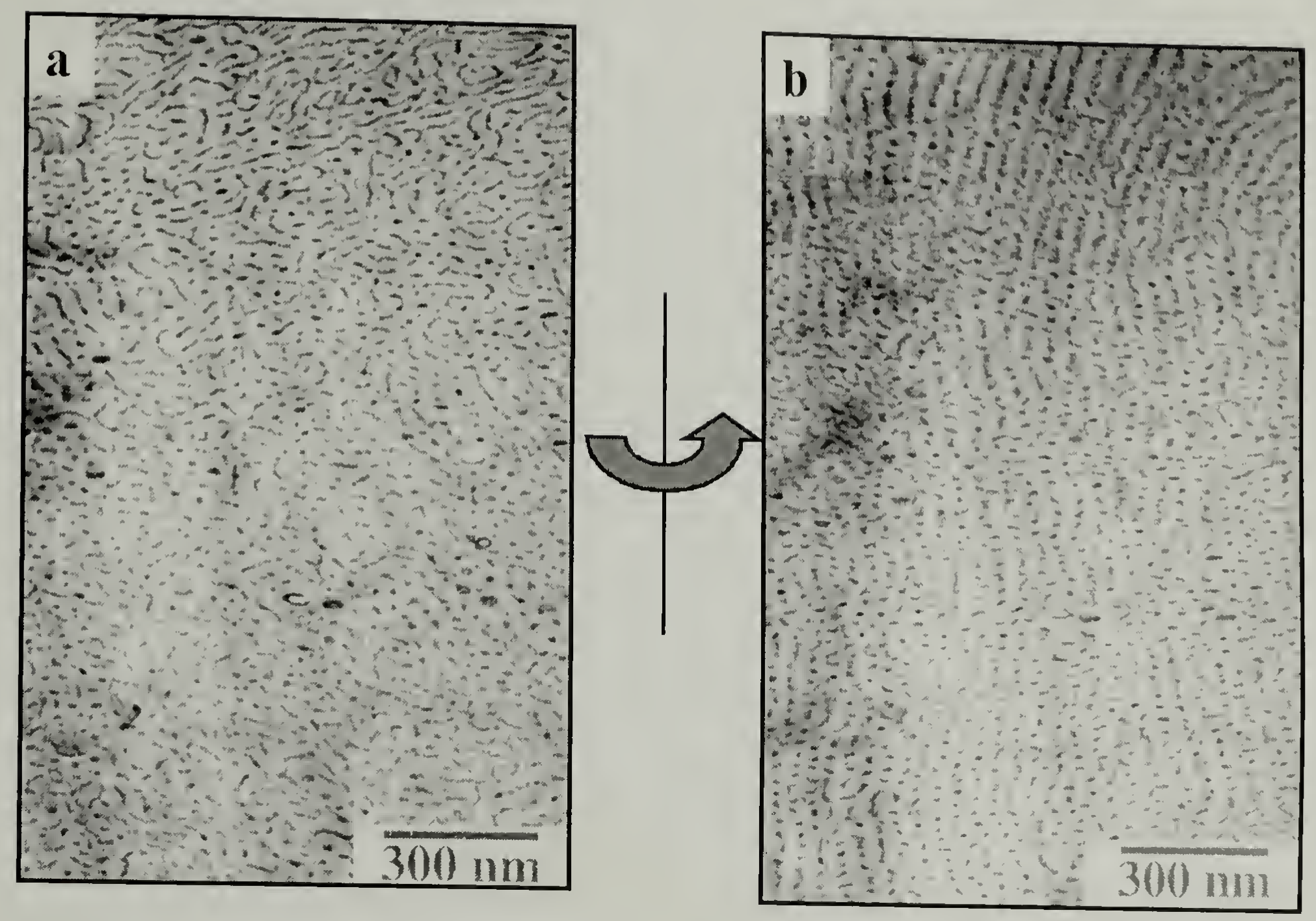

Figure 5.5. TEM tilt series of $\mathrm{I}_{3} \mathrm{~S}-86-\mathrm{F}$. (a) $0^{\mathrm{O}}$ tilt. (b) $30^{\circ}$ tilt about axis indicated. 


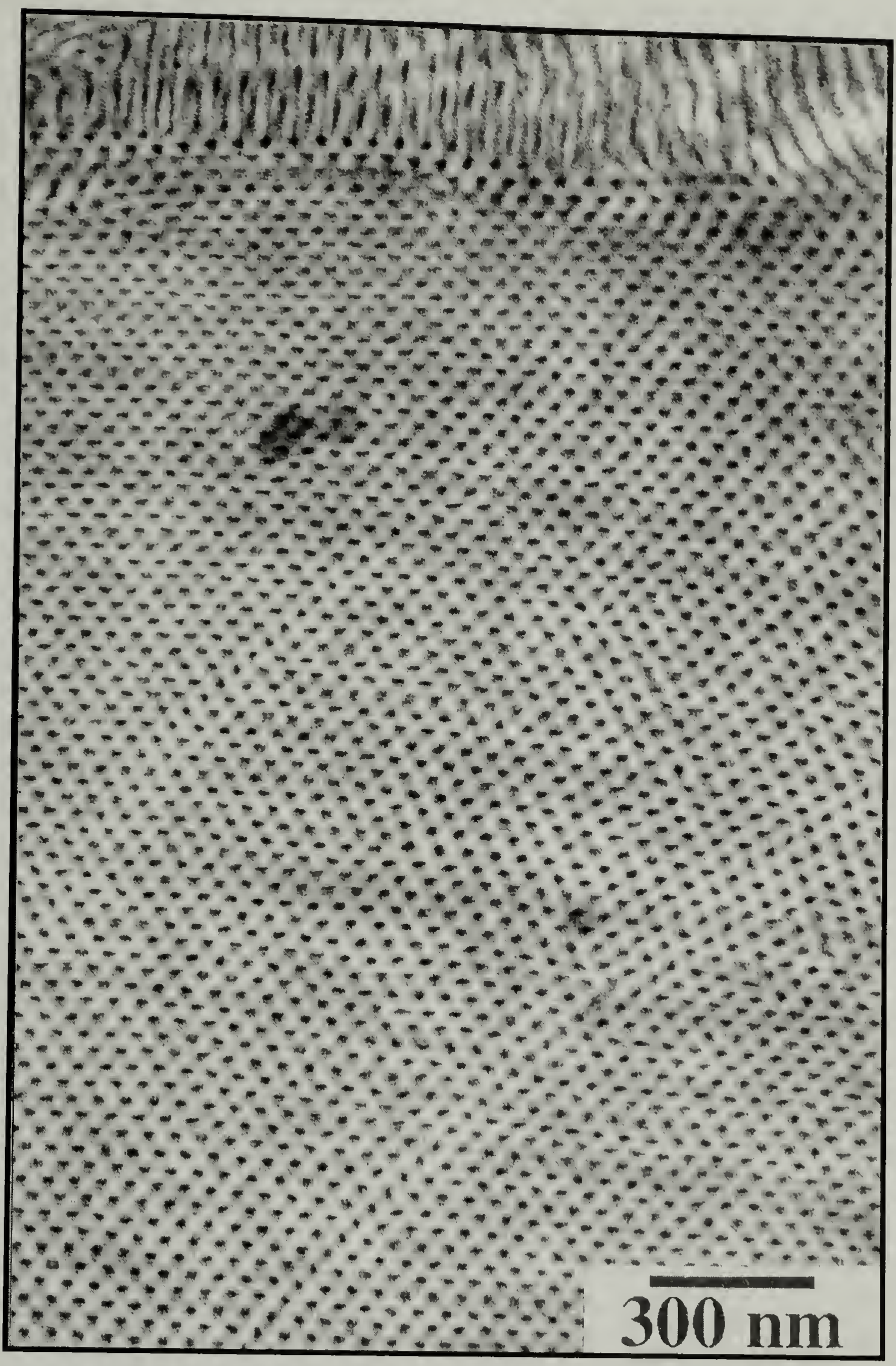

Figure 5.6. TEM micrographs of $\mathrm{I}_{3} \mathrm{~S}-86-\mathrm{G}$. 


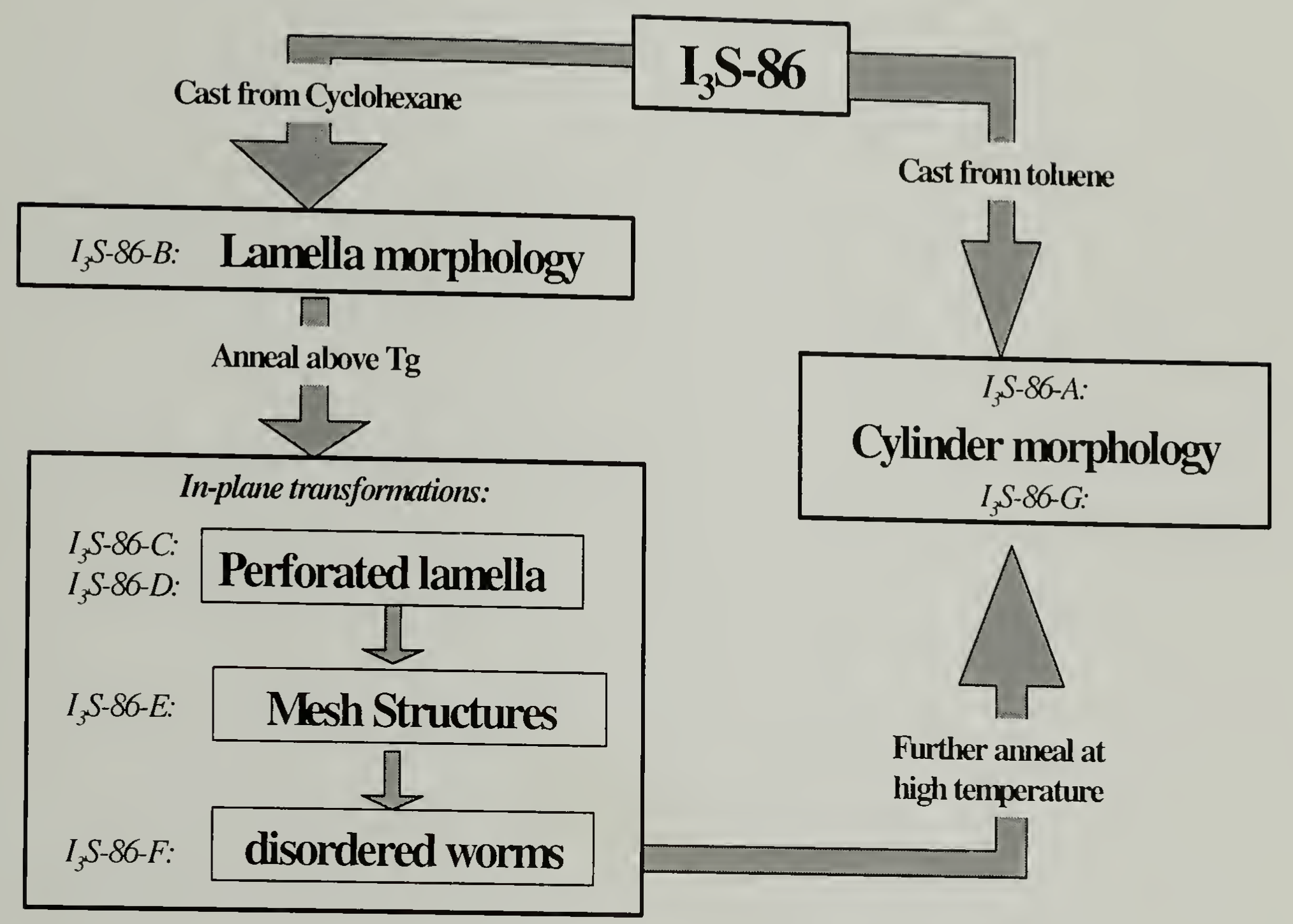

Figure 5.7. Morphology transformation sequence schematic for the lamella to cylinder transformation of $\mathrm{I}_{3} \mathrm{~S}-86$. 


\section{CHAPTER 6}

\section{CONCLUSIONS AND FUTURE WORK}

6.1 The Milner Model and the broken tilt lamella grain boundaries.

Although the Milner model had been proven to be generally correct in its predictions for the morphological behavior of miktoarm stars with relatively low asymmetry parameters, two important points of contention had arisen by the start of this work. The first issue was what was the true morphology transformation volume fraction at high asymmetry parameter and how well it agreed with the Milner's model. The second issue that had arisen was what is the reason for the observed increase of discrepancies between the theoretically predicted miktoarm star block copolymer morphology and the experimental observation as the molecules become more asymmetric.

Our study in $\mathrm{I}_{5} \mathrm{~S}$ miktoarm stars indicated that the disagreement between the theoretically calculated morphology transition volume fraction and the experiment observed morphology transition volume fraction is very significant at high asymmetry parameter. Based on the data, we proposed that as architectural asymmetry (arm number asymmetry) becomes large, geometric packing constraints also exert an important influence on morphology. Milner's calculation was based on the assumption of a rounded Wigner-Seitz cell surrounding each domain. ${ }^{1}$ In order for cylindrical and spherical domains to fill space, they must occupy true, space filling polyhedral Wigner-Seitz cells and deform to fill the corners of these cells. ${ }^{83,119}$ Therefore, the 
energies calculated with the rounded cell approximation (upon which Figure 4.5 is based) represent lower bounds. When the volume fraction of the component, which forms the core of the cylinder or sphere, is small (as in diblock copolymers), packing problems are not very important. However, as the molecule becomes more and more asymmetric, the volume fraction of the multi-arm component on the convex side of the interface may decrease to the point where it approaches the volume fraction of the interstitial space in a close packing of sphere. Thus we expect that geometric packing limits will prevent increasing shifts to higher $\phi$ of the true experimentally determined order-order transition lines in Figure 4.5 as $\varepsilon$ becomes large. A similar geometric packing limit will also apply to the cylindrical morphology. At these limits the order-order transitions will become vertical lines on the morphology diagram. On Figure 4.5, we have sketched the approximate positions, based on experimental data, of geometrical packing limits for spherical and cylindrical morphologies.

In the lamella forming sample, $\mathrm{I}_{5} \mathrm{~S}-58$, unusual chevron tilt grain boundary morphologies were observed, as shown in Figure 4.6. We postulate that the discontinuous nature of chevron tilt grain boundaries in $\mathrm{I}_{5} \mathrm{~S}-58$ results from an asymmetry in the energy penalties for interfacial bending due to the asymmetry inherent in the $\mathrm{I}_{5} \mathrm{~S}$ molecular architecture. Due to crowding inherent in packing five arms of PI on the same side of the interface and the fact that curving the interface toward the PI side will exacerbate chain stretching resulting from this crowding, it may be energetically more costly to bend the interfaces toward the PI domains than toward the PS domains in an $\mathrm{I}_{5} \mathrm{~S}$ architecture. By replacing the standard cheveron structure with a broken or discontinuous structure as illustrated in Figure 4.9b, it is possible to achieve the required 
tilt reorientation using only interfacial curvatures which put the five PI arms on the convex side of the interface. In order to do this, however, it is necessary to produce higher interfacial curvatures capable of generating semicylindrical end-caps on lamellae and isolated cylinders in the grain boundary plane.

\subsubsection{Proposed Research}

The results of these studies of miktoarm star morphological behavior lead to several interesting questions. First, regarding the effect of the geometric constriction, we sketched the approximate geometric limit position for sphere morphology based on the available experiment data. However, only six $\mathrm{I}_{5} \mathrm{~S}$ and six $\mathrm{I}_{3} \mathrm{~S}$ samples with lamella and cylinder morphologies have been examined. To get accurate value of the geometric limit for spheres, more $\mathrm{I}_{5} \mathrm{~S}$ and $\mathrm{I}_{3} \mathrm{~S}$ samples with lower PS volume fraction should be studied.

Another question concerning the geometric limits is the whether these $\mathrm{I}_{3} \mathrm{~S}$ and $\mathrm{I}_{5} \mathrm{~S}$ samples have reached these limit or they are just approaching these limit. To get accurate geometric limits for sphere and cylinder, a study of a series of much more asymmetric miktoarm star block copolymers molecules $\left(\mathrm{I}_{n} \mathrm{~S} n \pi 5\right)$ should be performed.

A third interesting question which arises from this study is the defect structures resulted from the specific asymmetric molecular architecture. An analysis of these boundaries based on the bending energy of the asymmetric molecular architecture was given. From the observation in Microscope, the broken chevron structures are usually observed at relatively high tilt angles. However, quantitative analyses have not been 
done. It will be interesting to further investigate the formation of discontinuous grain boundaries quantitatively in terms of tilt angle.

6.2 Investigation of morphology transformations using selective solvent approach.

Selective solvent can be used to control block copolymers' morphologies. ${ }^{2,32,44,103}$ Thermal annealing of these kinetically trapped morphologies can provide us information about the path of transformations among these morphologies. This experimental study showed that the lamella to cylinder transition goes though a series of in-plane transformations: perforated lamella, PI mesh, and PI worms. These transformations indicate perforated lamella not only involves in the lamella to Gyroid transitions ${ }^{35,37-}$ $39,46,49,50$ but also in lamella to cylinder transitions.

\subsubsection{Proposed research}

As discussed in the $1^{\text {st }}$ chapter, selective solvent approach provides many advantages and flexibilities in studying morphology transformations, which makes many interesting studies possible.

Due to the high MW of the polymer and resulting broad peak in SAXS, scattering method are not used. This study is based solely on microscopy evidence. Since TEM analysis only an limited portion of a sample, it is difficult to extract statistical results, like the dynamics of the transformation and its relation with MW, molecular architecture, temperature, etc, just from electron microscopy evidence.. Since scattering methods interact with a larger volume, they provide data with good statistical confidence. 
However, to get high quality scattering date about the in plane transformation and out of plane transformations, it is important to get aligned unstable lamella morphology at the first step. Since the desired lamella morphology is a kinetically trapped, unstable morphology, the typical method, melt state shear alignment is not applicable. To achieve this purpose, a novel roll caster, as shown in Figure 6.1, has been designed and constructed. Roll caster was developed in Thomas group to solvent cast block copolymer into globally oriented morphology. ${ }^{15,116}$ If we combine this idea with selective solvent, it is possible to cast block copolymer into unstable oriented morphologies. For our purpose, the designed roll caster has the following improvements over the reported roll casters.

1 The 4-pulley system design uses only one motor, which is compact and accurate in rotation speed control.

2 By rotating the wing-nut to adjust the distance between two cylinders, the thickness of the film can be controlled.

3 The curved bottom of the new design is material efficient, which is suitable for casting small amount of materials.

4 All Teflon construction makes it easy to retrieve the sample and to clean the instrument.

Using selective solvent, it is possible get kinetically trapped, yet globally oriented structures which facilitate the study irreversible morphology transformations.

Another interesting topic arise from this study is structure-property relationship. Previous study investigating the effects of morphology over properties was obtained by 
extrapolations. Since many factors, like total molecular weight, molecular weight of the plastic block, molecular weight of the rubber block, volume fraction, annealing conditions, etc, all affect block copolymer properties, the extrapolations to relate morphology with properties are not accurate.. However, using selective solvent approach, we can obtain different morphologies with exactly same materials, thus we will be able to obtain precise information on morphology-properties relationship.

\subsection{Morphology of Miktoarm star block copolymer / homopolymer blends.}

Here, we use well-defined $\mathrm{A}_{2} \mathrm{~B}, \mathrm{~A}_{2} \mathrm{~B}_{2}$ star shaped block copolymer, which are the structure units of more complex graft block copolymer systems,,$^{3,4,6,31}$ as the model systems to study the effects of molecular architecture on graft block copolymer / homopolymer blends. The first type, $\mathrm{I}_{2} \mathrm{~S}$ block copolymers, which have 2 low polydispersity (PDI) polyisoprene arms and one low PDI polystyrene arm joint at a single junction point, has an asymmetry, non-linear molecular architecture. ${ }^{2,87}$ Previously reported linear diblock copolymer / homopolymer blend systems showed that the orderorder transitions (OOTs) occur at about the same volume fractions as in pure linear diblock copolymers. The OOT occurs at the same volume fraction regardless of the direction from which it is approached, i.e. blending homopolymer A with a diblock which forms A cylinders in a B matrix to push it toward lamella or blending B homopolymer with a lamellar diblock to push it back toward cylinders. ${ }^{17,18,20}$ This study shows that when blending a homopolymer with an $\mathrm{I}_{2} \mathrm{~S}$ block copolymer that the OOTs split so that they occur at different volume fractions depending upon whether they are approached by blending homopolymer into the two arm or the one arm side of the block copolymer 
interface. Perforated lamellar morphology is obtained in a blend of homopolystyrene (hPS) and a lamella forming single graft block copolymer, and it is found stable to thermal annealing.

The second type, $\mathrm{I}_{2} \mathrm{~S}_{2}$ block copolymers, which have 2 low PDI polyisoprene arms and two low PDI polystyrene arms joint at a single junction point, has an symmetry, non-linear molecular architecture. ${ }^{9}$ Although $\mathrm{I}_{2} \mathrm{~S}_{2}$ block copolymers have symmetry molecular architecture, their blends with homopolymer also show strong shifts in the order-order transition volume fractions. Inverted phase is also observed in one of the blends with high homopolymer concentration. A slow co-casting procedure was developed to get single crystal structure of Gyroid morphology. The amazing scattering patterns provide the best evidence for Gyroid morphology observed so far.

\subsubsection{Proposed Research}

Using a slow casting procedure, one of the blends form amazingly ordered Gyroid structure. The scattering pattern proved the $I a \overline{3} d$ symmetry of the structure. However, the inter-material dividing surface of Gyroid structure has not been well defined. The excellent SAXS data provides opportunities to illustrate the detail of the structure.

Another interesting question arising from the study is the relation between casting condition and long-range orders. As discussed above, though slow casting, a couple gyroid samples formed "single crystal" like long-range order. Perfect single crystals are usually observed in atom and molecular level self-assemblies with each consisting of materials perfectly the same. In nano-scale self-assemblies, the repeat unit usually 
consists of hundreds to tens of thousands of atoms and the component molecules are not necessarily identical. Fluctuations and defects lead to imperfections and prevent the formation of large single crystal. All the cylinder or lamella blend samples are processed at the same conditions, but none of them have nearly the same degree of ordering as the Gyroid samples. This observation implies the defects structure in the inter-penetration network of Gyroid have relatively higher energies or kinetically easier to eliminate than that of lamella and cylinder morphologies. Achieving long-range order in nano-scale material is very important for many potential applications, like photonic material, nanoreactors, etc. A further study of the casting condition and the resulting long-range order or block copolymer and block copolymer / homopolymer blend systems will be very interesting. 

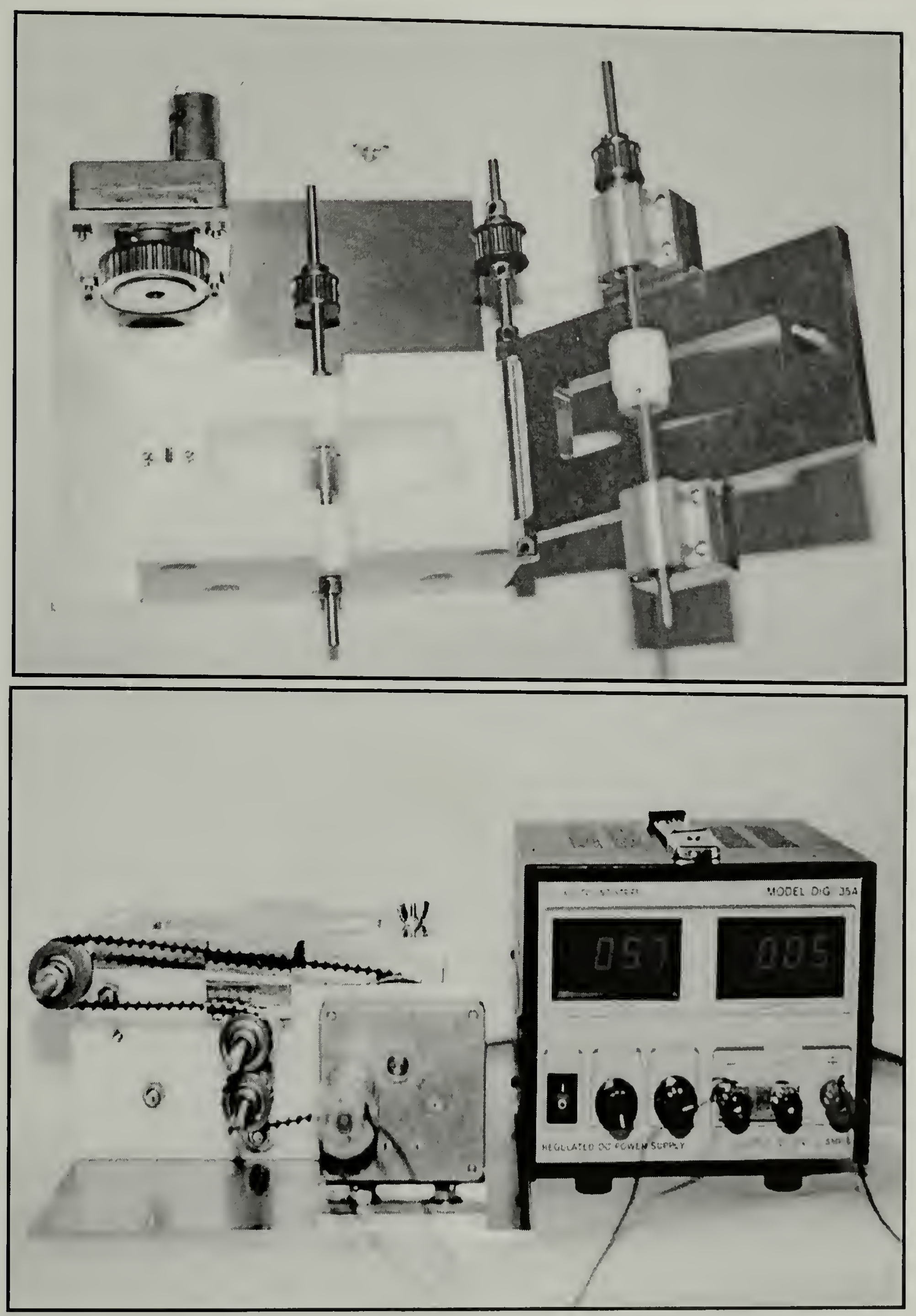

Figure 6.1. Top view and side view of the "roll caster". 


\section{BIBLIOGRAPHY}

1)Hadjichristidis, N.; Iatrou, H.; Behal, S. K.; Chludzinski, J. J.; Disko, M. M.; Garner, R. T.; Liang, K. S.; Lohse, D. J.; Milner, S. T. Macromolecules 1993, 26, 58125815 .

2)Pochan, D. J.; Gido, S. P.; Pispas, S.; Mays, J. W. Macromolecules 1996, 29, 5099.

3)Lee, C.; Gido, S. P.; Poulos, Y.; Hadjichristidis, N.; Beck Tan, N.; Trevino, S. F.; Mays, J. W. Journal of Chemical Physics 1997, 107, 6460.

4)Lee, C.; Gido, S. P.; Poulos, Y.; Hadjichristidis, N.; Beck Tan, N.; Trevino, S. F.; Mays, J. W. Polymer 1997, 39, 4631-4638.

5)Lee, C.; Gido, S. P.; Pitsikalis, M.; Mays, J. W.; Beck Tan, N.; Trevino, S. F.; Hadjichristidis, N. Macromolecules 1997, 30, 3732-3738.

6)Lee, C. "The Morphological Behavior of Model Graft Copolymers," University of Massachusetts, 1998

7)Matsushita, Y.; Noda, I. Macromolecular Symposia 1996, 106, 251-257.

8)Beyer, F. L.; Gido, S. P.; Veils, G.; Hadjichristidis, N.; Tan, N. B. Macromolecules 1999, 32, 6604-6607.

9)Beyer, F. L.; Gido, S. P.; Uhrig, D.; Mays, J. W.; Tan, N. B.; Trevino, S. F. Journal of Polymer Science 1999, 37, 3392-3400.

10)Beyer, F. L.; Gido, S. P.; Poulos, Y.; Avgeropoulos, A.; Hadjichristidis, N. Macromolecules 1997, 30, 2373.

11)Milner, S. T. Macromolecules 1994, 27, 2333.

12)Tselikas, Y.; Hadjichristidis, N.; Lescanec, R. L.; Honeker, C. C.; Wohlgemuth, M.; Thomas, E. L. Macromolecules 1996, 29, 3390.

13)Koizumi, S.; Hasegawa, H.; Hashimoto, T. Macromolecules 1994, 27, 6532-6540.

14)Hashimoto, T.; Tanaka, H.; Hasegawa, H. Macromolecules 1990, 23, 4378-4386.

15)Tanaka, H.; Hasegawa, H.; Hashimoto, T. Macromolecules 1991, 24, 240.

16)Tanaka, H.; Hashimoto, T. Macromolecules 1991, 24, 5713.

17)Winey, K. I.; Thomas, E. L.; Fetters, L. J. Macromolecules 1992, 25, 2645. 

18)Winey, K. I.; Thomas, E. L.; Fetters, L. J. Journal of Chemical Physics 1991, 95,
9367.

19)Winey, K. I.; Thomas, E. L.; Fetters, L. J. Macronolecules 1991, 24, 6128-6188.

20)Winey, K. I.; Thomas, E. L.; Fetters, L. J. Macromolecules 1992, 25, 422.

21)Bates, F. S.; Maurer, W.; Lodge, T. P.; Schulz, M. F.; Matsen, M. W. Physical Review Letters 1995, 75, 4429-4432.

22)Disko, M. M.; Liang, K. S.; Behal, S. K.; Roe, R. J.; Jeon, K. J. Macromolecules 1993, 26, 2983-2986.

23)Norman, D. A.; Kane, L.; White, S. A.; Smith, S. D.; Spontak, R. J. Journal of Material Science Letters 1998, 17, 545-549.

24)Laurer, J. H.; Hajduk, D. A.; Dreckotter, S.; Smith, S. D.; Spontak, R. J. Macromolecules 1998, 31, 7546-7549.

25)Lesance, R. L.; Fetters, L. J.; Thomas, E. L. Macromolecules 1998, 31, 1680-1685.

26)Braun, D.; Fischer, M.; Hellmann, G. P. Polymer 1996, 37, 3871.

27)Amorim, M. C. V.; Oliveira, C. M. F.; Tavares, M. I. B. J. Appl. Polym. Sci. 1996, 61, 2245.

28)Feng, H.; Tian, J.; Ye, C. J. Appl. Polym. Sci. 1996, 61, 2265.

29)Yanagase, A.; Ito, M.; Yamamoto, N.; Ishikawa, M. J. Appl. Polym. Sci. 1996, 60, 87.

30)Miyata, T.; Takagi, T.; Uragami, T. Macromolecules 1996, 29, 7787.

31)Beyer, F. L.; Gido, S. P.; Buschl, C.; Iatrou, H.; Uhrig, D.; Mays, J. W.; Chang, M. Y.; Garetz, B. A.; Balsara, N. P.; Beck Tan, N.; Hadjichristidis, N. Macromolecules 2000, 33, 2039-2048.

32)Sakurai, S.; Kawada, H.; Hashimoto, T.; Fetters, L. J. Macromolecules 1993, 26 , 5796.

33)Sakurai, S.; Hashimoto, T.; Fetters, L. J. Macromolecules 1996, $29,5769$.

34)Hajduk, D. A.; Gruner, S. M.; Rangarajan, P.; Register, R. A.; Fetters, L. J.; Honeker, C.; Albalak, R. J.; Thomas, E. L. Macromolecules 1994, 27, 490.

35)Hajduk, D. A.; Ho, R.-M.; Hillimyer, M. A.; Bates, F. S.; Almdal, K. J. Phys. Chem. B 1998, 1356-1363.

36)Hajduk, D. A.; Harper, P. E.; Gruner, S. M.; Honeker, C. C.; Thomas, E. L.; Fetters, L. J. Macromoles 1995, 28, 2570-2573. 
37)Khandpur, A. K.; Forster, S.; Bates, F. S. Macromolecules 1995, 28, 8796-8806.

38)Förster, S.; Khandpur, A. K.; Zhao, J.; Bates, F. S.; Hamley, I. W.; Ryan, A. J.; Bras, W. Macromolecules 1994, 27, 6922.

39)Vigilid, M. E.; Almdal, K.; Mortensen, K.; Hamley, I. W.; Fairclough, J. P. A.; Ryan, A. J. Macromolecules 1998, 31, 5702-5716.

40)Almdal, K.; Koppi, K. A.; Bates, F. S.; Mortensen, K. Macromolecules 1992, 25, 1743-1751.

41)Atkins, P. W. Physical Chemistry; 5th ed.; W. H. Freeman and Company: New York, 1994.

42)Bates, F. S.; Fredrickson, G. H. In Annual Review of Physical Chemistry; Strauss, H. L., Babcock, G. T., Moore, C. B., Eds., 1990; Vol. 41, p 525.

43)Fredrickson, G. H.; Bates, F. S. Annual Review of Materials Science Polymers 1996, 26.

44)Thomas, E. L.; Alward, D. B.; Kinning, D. J.; Martin, D. C.; Handlin, D. L., Jr.; Fetters, L. J. Macromolecules 1986, 19, 2197.

45)Hasegawa, H.; Tanaka, H.; Yamasaki, K.; Hashimoto, T. Macromolecules 1987, 20 , 1651.

46)Hajduk, D. A.; Harper, P. E.; Gruner, S. M.; Honeker, C. C.; Kim, G.; Thomas, E. L.; Fetters, L. J. Macromolecules 1994, 27, 4063.

47)Zhao, J.; Majumdar, B.; Schulz, M. F.; Bates, F. S.; Almdal, K.; Mortensen, K.; Hajduk, D. A.; Gruner, S. M. Macromolecules 1996, 29, 1204-1215.

48)Thomas, E. L.; Anderson, D. M.; Henkee, C. S.; Hoffman, D. Nature 1988, 334, 598601.

49)Hajduk, D. A.; Takenouchi, H.; Hillmyer, M. A.; Bates, F. S.; Vigild, M. E.; Almdal, K. Macromolecules 1997, 30, 3788-3795.

50)Hamley, I. W.; Gehlsen, M. D.; Khandpur, A. K.; Koppi, K. A.; Rosedale, J. H.; Schulz, M. F.; Bates, F. S.; Almdal, K.; Mortensen, K. J. Phys. 2 France 1994, 4, 2161-2186.

51)Burger, C.; Micha, M. A.; Oestereich, S.; S.Forster; Antonietti, M. Europhysics letters $1998,42,425-429$.

52)Ahn, J.-H.; Zin, W.-C. Macromolecules 2000, 33, 641-644.

53)Hashimoto, T.; Koizumi, S.; Hasegawa, H.; Izumitani, T.; Hyde, S. T. Macromolecules 1992, 25, 1443-1439. 

54)Yang, L.; Gido, S. P.; Mays, J. W.; Pispas, S.; Hadjichristidis, N. Macromolecules
2001, 34, 4235-4243.

55)Semenov, A. N. Soviet Physics JETP 1985, 61, 733.

56)Fredrickson, G. H. Macromolecules 1991, 24, 3456.

57)Qi, S.; Wang, Z.-G. Physical review letters 1996, 76, 1679-1682.

58)Qi, S.; Wang, Z.-G. Physical Review E 1997, 55, 1682-1697.

59)Qi, S.; Wang, Z.-G. Macromolecules 1997, 30, 4491-4497.

60)Matsen, M. W.; Bates, F. S. Macromolecules 1996, 29, 1091.

61)Young, R. J.; Lovell, P. A. Introduction to Polymers; 2nd ed.; Chapman \& Hall: New York, 1991.

62)Helfand, E.; Tagami, Y. Journal of Chemical Physics 1972, 57, 1812-1813.

63)Helfand, E.; Tagami, Y. Journal of Chemical Physics 1972, 56, 3592-3601.

64)Helfand, E. Journal of Chemical Physics 1975, 62, 999-1005.

65)Helfand, E. Macromolecules 1975, 8, 552-556.

66)Helfand, E. Accounts of Chemical Research 1975, 8, 295-299.

67)Helfand, E. Journal of Chemical Physics 1975, 63, 2192-2198.

68)Helfand, E.; Sapse, A. M. Journal of Chemical Physics 1975, 62, 1327-1331.

69)Helfand, E.; Wasserman, Z. R. Macromolecules 1976, 9, 879.

70)Helfand, E.; Wasserman, Z. R. Polymer Engineering and Science 1977, 17, 582.

71)Helfand, E.; Wasserman, Z. R. Macromolecules 1978, 11, 960-966.

72)Helfand, E.; Wasserman, Z. R. Macromolecules 1980, 13, 994-998.

73)Leibler, L. Macromolecules 1980, 13, 1602.

74)Schulz, M. F.; Bates, F. S.; Almdal, K.; Mortensen, K. Physical Review Letters 1994, 73, 86-89.

75)Milner, S. T.; Witten, T. A. Journal de Physique 1988, 49, 1951.

76)Milner, S. T.; Witten, T. A.; Cates, M. E. Macromolecules 1988, 21, 2610. 
77)Milner, S. T.; Witten, T. A.; Cates, M. E. Europhysics Letters 1988, 5, 413-418.

78)Milner, S. T.; Wang, Z. G.; Witten, T. A. Macromolecules 1989, 22, 489.

79)Milner, S. T.; Witten, T. A.; Cates, M. E. Macromolecules 1989, 22, 853.

80)Milner, S. T. J. Chem. Soc. Faraday Trans. 1990, 86, 1349-1353.

81)Milner, S. T. Science 1991, 251, 905.

82)Milner, S. T. Journal of Polymer Science: Part B: Polymer Physics 1994, 32, $2743-$ 2755.

83)Olmsted, P. D.; Milner, S. T. Macromolecules 1998, 31, 4011-4022.

84)Olmsted, P. D.; Milner, S. T. Physical Review Letters 1994, 72, 936.

85)Matsen, M. W.; Bates, F. S. Macromoles 1996, 29, 7641-7644.

86)Matsen, M. W.; Bates, F. S. Journal of Polymer Science Pt. B: Polymer Physics 1997, $35,945-952$.

87)Pochan, D. J.; Gido, S. P.; Pispas, S.; Mays, J. W.; Ryan, A. J.; Fairclough, J. P. A.; Hamley, I. W.; Terrill, N. J. Macromolecules 1996, 29, 5091-5098.

88)Pochan, D. J.; Gido, S. P.; Zhou, J.; Mays, J. W.; Whitmore, M.; Ryan, A. J. Journal of Polymer Science, Polymer Physics 1997, 35, 2629-2643.

89)Gido, S. P.; Lee, C.; Pochan, D. J.; Pispas, S.; Mays, J. W.; Hadjichristidis, N. Macromolecules 1996, 29, 7022.

90)Tselikas, Y.; Iatrou, H.; Hadjichristidis, N.; Liang, K. S.; Mohanty, K.; Lohse, D. J. J. Chem. Phys. 1996, 105, 2456.

91)Shull, K. R.; Winey, K. I. Macromolecules 1992, 25, 2637.

92)Laverdure, K.; Gido, S. P.; Pispas, S.; Mays, J. W. In preparation.

93)Oster, G.; Riley, D. P. Acta Crystyllographica 1952, 5, 272-276.

94)Oster, G.; Riley, D. P. Acta Crystallographica 1952, 5, 1-6.

95)Matsen, M. W. Macromolecules 1995, 28, 5765-5773.

96)Zhu, Y.; Gido, S. P. In preparation.

97)Kinning, D. J.; Winey, K. I.; Thomas, E. L. Macromolecules 1988, 21, 3502-3506.

98)Bates, F. S.; Berney, C. V.; Cohen, R. E. Macromolecules 1983, 16, 1101. 
99)Alward, D. B.; Kinning, D. J.; Thomas, E. L. macromolecules 1986, 19, 215-224.

100)Herman, D. S.; Kinning, D. J.; Thomas, E. L.; Fetters, L. J. Macromolecules 1987, 20, 2940-2942.

101)Iatrou, H.; Hadjichristidis, N. Macromolecules 1993, 26, 2479-2484.

102)Yang, L.; Hong, S.; Gido, S. P.; Veils, G.; Hadjichristidis, N. in press Macromolecules 2001.

103)Hanley, K. J.; Lodge, T. P.; Huang, C.-I. Macromolecules 2000, 33, 5918-5931.

104)Crystallography, I. U. o. Internation Tables For X-Ray Crystallography; 2nd Edition ed.; Birmingham, Eng.:, 1965.

105)Turner, C. M.; Sheller, N. B.; Foster, M. D.; Lee, B.; Corona-Galvin, S.; Quirk, R. P.; Annis, B.; Lin, J.-S. Macromolecules 1998, 31, 4372-4375.

106)Lee, C.; Gido, S. P.; Poulos, Y.; Hadjichristidis, N.; Beck Tan, N.; Trevino, S. F.; Mays, J. W. Polymer 1998, 39, 4631-4638.

107)Xenidou, M.; Beyer, F. L.; Gido, S. P.; Hadjichristidis, N.; Beck Tan, N. Macromolecules 1998, 31, 7659-7667.

108)Gehlsen, M. D.; Bates, F. S. Macromolecules 1994, 27, 3611.

109)Lin, C. C.; Jonnalagadda, S. V.; Kesani, P. K.; Dai, H. J.; Balsara, N. P. Macromolecules 1994, 27, 7769-7780.

110)Velis, G.; Hadjichristidis, N. Macromolecules 1999, 32, 534-536.

111)Polymer Handbook; 3rd ed.; Wiley-Interscience: New York, 1989.

112)Cowie, J. M. G. In Developments in Block Copolymers - 1; Goodman, I., Ed.; Applied Science Publishers: New York, 1982; pp 1-38.

113)Keller, A.; Odell, J. A. Processing, Structure and Properties of Block Copolymer; Elsevicr Applied Science: New York, 1985.

114)Hadziioannou, G.; Mathis, A.; Skoulios, A. Colloid and Polymer Science 1979, 257, 22.

115)Albalak, R. J.; Thomas, E. L. Journal of Polymer Science, Polym. Pliys. Ed. 1993, 31,37 .

116)Albalak, R. J.; Thomas, E. L. Journal of Polymer Science, Polym. Phys. Ed. 1994, 32,341 . 
117)Grayer, V.; Dormidontova, E. E.; Hadziioannou, G.; Tsitsilianis, C. Macromolecules 2000, 33, 6330-6339.

118)Matsen, M. W.; Gardiner, J. M. J. Chem. Phys 2000, 113, 1673-1676.

119)Gido, S. P.; Schwark, D. W.; Thomas, E. L.; Goncalves, M. Macromolecules 1993, 26, 2636.

120)Gido, S. P.; Thomas, E. L. Macromolecules 1994, 27, 6137.

121)Polis, D. L.; Winey, K. I. Macromolecules 1996, 29, 8180-8187.

122)Polis, D. L.; Winey, K. I. Macromolecules 1998, 31, 3617-3625.

123)Qiao, L.; Winey, K. I. Macromolecules 2000, 33, 851-856.

124)Matsen, M. W. J. Chem. Phys. 1997, 107, 8110-8119.

125)Netz, R. R.; Andelman, D.; Schick, M. Phys. Rev. Lett. 1997, 79, 1058-1061.

126)Burgaz, E.; P.Gido, S. Macromolecules 2000, 33, 8739-8745.

127)Yang, L.; Hong, S.; Gido, S. P.; Uhrig, D.; Mays, J. W. Submitted to Macromolecules 2001.

128)McLeish, T. C. B.; Milner, S. T. Advances in Polymer Science 1999, 143, 195-256. 


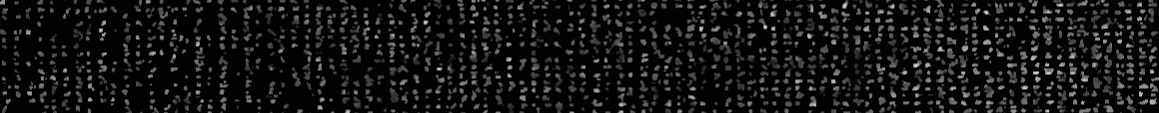

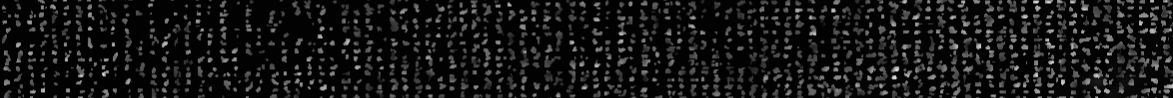

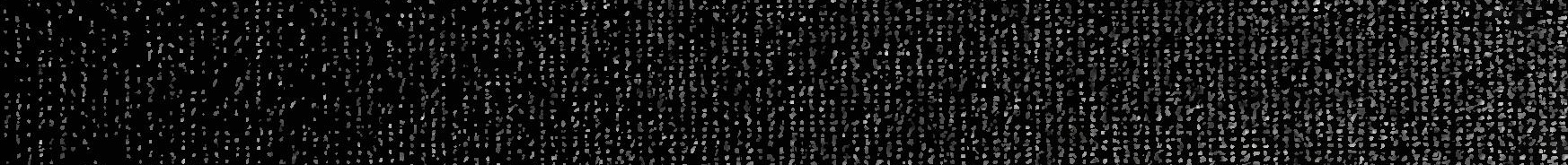
6. -

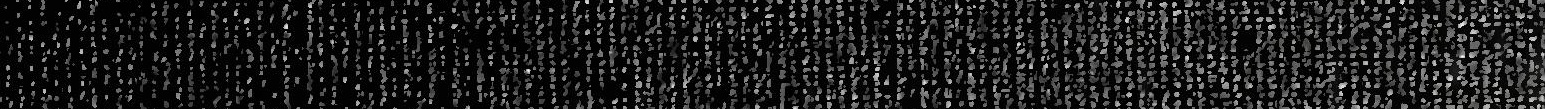

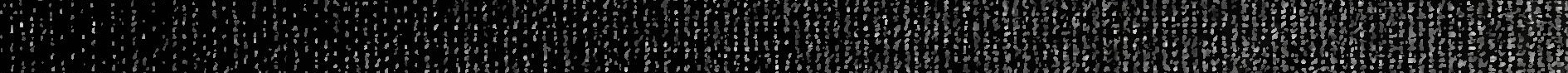
H.

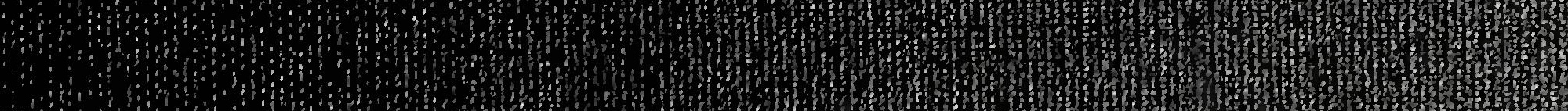

